\title{
Nitric oxide and vascular remodeling
}

Citation for published version (APA):

Schulten, H. M. (2004). Nitric oxide and vascular remodeling. [Doctoral Thesis, Maastricht University]. Datawyse / Universitaire Pers Maastricht. https://doi.org/10.26481/dis.20040416hs

Document status and date:

Published: 01/01/2004

DOI:

10.26481/dis.20040416hs

Document Version:

Publisher's PDF, also known as Version of record

\section{Please check the document version of this publication:}

- A submitted manuscript is the version of the article upon submission and before peer-review. There can be important differences between the submitted version and the official published version of record.

People interested in the research are advised to contact the author for the final version of the publication, or visit the DOI to the publisher's website.

- The final author version and the galley proof are versions of the publication after peer review.

- The final published version features the final layout of the paper including the volume, issue and page numbers.

Link to publication

\footnotetext{
General rights rights.

- You may freely distribute the URL identifying the publication in the public portal. please follow below link for the End User Agreement:

www.umlib.nl/taverne-license

Take down policy

If you believe that this document breaches copyright please contact us at:

repository@maastrichtuniversity.nl

providing details and we will investigate your claim.
}

Copyright and moral rights for the publications made accessible in the public portal are retained by the authors and/or other copyright owners and it is a condition of accessing publications that users recognise and abide by the legal requirements associated with these

- Users may download and print one copy of any publication from the public portal for the purpose of private study or research.

- You may not further distribute the material or use it for any profit-making activity or commercial gain

If the publication is distributed under the terms of Article $25 \mathrm{fa}$ of the Dutch Copyright Act, indicated by the "Taverne" license above, 
NITRIC OXIDE AND VASCULAR REMODELING 
Copyright $(\mathcal{O H e n n y}$ Schulten, Matastrich 2004

ISBN 9052784094

Vormgeving an druk. Datawyse | Universitaire Pers Mastricht

Omslag: G.E. Fazzi 


\title{
NITRIC OXIDE AND VASCULAR REMODELING
}

\author{
PROEFSCHRIFT \\ ter verkrijging van de graad van doctor \\ aan de Universiteit Maastricht, \\ op gezag van de Rector Magnificus, \\ Prof, mr. G.P.M.F. Mols, \\ volgens het besluit van her College van Decanen, \\ in het openbaar te verdedigen \\ op vrijdag 16 april 2004 om 12.00 uur \\ door \\ Henny Schulten \\ geboren te Tilburg
}

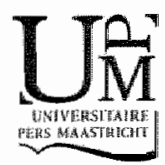




\section{Promotores:}

Prof. dr. H.J.J. Wellens

Prof. dr. M.J.A.P. Daemen

Co-promotor:

Dr. E.D. de Muinck

\section{Beoordelingscommissie:}

Prof. dr. A.P.G Hoeks (voorzitter)

Prof. dr. C.E. Blanco

Prof. dr. M.M. Kocks (Universiteit Antwerpen, België)

Prof. dr. A.J. Rabelink (Universiteit Leiden)

Prof. dr. I. Waltenberger

Financial support by the Netherlands Heart Foundation for the publication of this thesis is gratefully acknowledged.

Additional support was granted by: Rescar Foundaton Maastricht. 


\section{Table of contents}

CHAPTER I

Introduction 7

CHAPTER 2

Reduced endothelium-dependent relaxations and impaired acute flowinduced vasodilation do not compromise chronic flow-related arterial remodeling in hyperlipidemic mice. Submithed. 41

CHAPT"ER 3

Effects of estrogen on the vascular injury response in the estrogen receptor $\alpha, \beta$ (double) knockout mice. Circ. Res. 2001,89:534-39. 59

CHAPTER 4

Nitric oxide synthase(s) mediate the vascular protective effect of estrogen in the mouse carotid artery injury model. Submitted. 75

CHAPTER 5

Disruprion of the iNOS gene abolishes estrogen-mediated protection against vascular injury in mice. Submitted. $\quad 87$

CHAPTER 6

Endothelial nitric oxide synthase inhibits neointimal hyperplasia after vascular injury in hyperlipidemic mice. Submitted. 99

CHAPTER 7

General discussion 115

SUMMARY $\quad 129$

SAMENVATTTNG 131

DANKWOORD 133

CURRICULUM VITAE 137

LIST OF PUBLICATIONS 139 



\section{CHAPTER 1}

\section{Introduction}

It is known that the endothelium is not simply an inert barrier between the blood and the vascular smooth muscle. In addition to its barrier funcrion, the endothelium is actively involved in the regulation of vascular tone and blood flow, in maintaining a balance between blood fluidity and thrombosis, in modulating control by perivascular nerves and in the regulation of cell growth within the vessel wall. One of the most important molecules involved in these processes is nitric oxide (NO), which is constitutively produced by endothelial NO synthase (eNOS).

Hypercholesterolemia and arherosclerosis are associated with endorhelial dysfunction which causes a shift in the balance between locally produced vasodilators, such as $\mathrm{NO}$, prostacyclin, bradykinin and endothelium-derived hyperpolarizing factor(s) and constrictors such as endothelin. As a consequence, endothelial dysfunction is characterized by decreased vasodilation and increased vasoconstriction in response to various stimuli. Another hall mark of endothelial dysfunction is the loss of atheroprotective properties of the endothelial lining. Hence, persisting endothelial dysfunction will contribute to atherosclerotic plaque formation. One of the mechanisms that compensate for vessel wall thickening caused by plaque accumulation is outward remodeling of the vessel. When outward remodeling can no longer compensate for vessel wall thickening, luminal narrowing ensues causing impaired perfusion and oxygenation of the tissue down stream from the obstruction. When medical therapy is unsuccessful in alleviaring the symproms of tissue hypoxia, invasive strategies are mandated that either bypass the obstruction with an arterial of venous graft or abolish it by dilaring the artery at the site of the obstruction. The surgical approach is termed coronary artery bypass graft (CABG) surgery and abolishment of the stenosis entails a catheter-based approach known as percutaneous intervention. Currently, stents are implanted in $>90 \%$ of percutaneous intervention cases. Vessel narrowing recurs (restenosis) after both surgical and percuatenous intervention and the new lesion is the result of a wound healing response triggered by the vascular trauma caused by $\mathrm{CABG}$ or percutaneous intervention. It is characterized by a combination of smooth muscle cell proliferation and inward remodeling.

In this thesis the main hyporhesis is tested that NO inhibits the remodeling response after vascular injury. In addition the effects of two potentiarors of NO 
production are tested in the context of vascular injury and atherosclerosis. The specific hypotheses are that:

- endothelium-dependent flow-mediated outward remodeling is impaired in a hyperlipidemic mouse model with endothelial dysfunction.

- estrogen's ability to inhibir vascular remodeling after arterial injury is mediated via eNOS-derived NO.

- local NOS-dependent delivery of NO inhibits vascular remodeling after arterial damage in hyperlipidemic mice (A) an effect that is fortified by systemic application of folate, a cofactor for eNOS enzyme (B).

\section{NITRIC OXIDE}

In 1980, Furchgort (Nobel prize 1998) and Zawadzki observed that the presence of an intact vascular endothelium is a prerequisite for acetylcholine-induced relaxation of smooth muscle cells. ${ }^{2}$ Later this relaxation was shown to occur in a $\mathrm{Ca}^{2+}$ dependent manner via stimulation of the soluble isoform of guanylate cyclase. The endothelium-dependent relaxing factor (EDRF) was identified as the free radical gas $\mathrm{NO}$, which is highly lipophilic and chemically reactive, allowing it to bind to a large variety of chemical moieties. ${ }^{2-4}$ It has become apparent that the simple molecule $\mathrm{NO}$ plays an important role in regulating many biological functions such as vascular tone, ${ }^{5}$ platelet activation ${ }^{6}$ and neurotransmission. 7,8 Also, NO has been shown to be an important immunoregulatory molecule with cytostatic and cytotoxic properties. $9,10 \mathrm{NO}$ is a gas that is poorly soluble in water. It has a short half-life of approximately 10 to 60 seconds in tissue, because it readily reacts with oxygen, superoxide radicals, and transition metals such as iron and copper. ${ }^{11,12}$ Consequently, NO cannot function at a distance, when it enters the blood stream it combines rapidly with oxyhemoglobin to form methemoglobin and nitrate $\left(\mathrm{NO}_{3}\right) \cdot{ }^{13}$ However, $\mathrm{NO}$ is particularly useful as an intracellular messenger because it can easily diffuse through most cells and tissues. ${ }^{\text {Id }}$

\section{No-chemistry}

$\mathrm{NO}$ is generated from the rerminal guanidino-nirrogen of $\mathrm{L}$-arginine by an $\mathrm{N}^{10}$-hydroxyl-L-arginine intermediate yielding citrulline. 11,15 This reaction is catalyzed by a NO synthase (NOS):

$$
\text { L-arginime }+n N A D P H+m \mathrm{O}_{2} \stackrel{\mathrm{NOS}}{\rightarrow} \text { citmulline }+\mathrm{NO}+n N A D P+.
$$

NOS contains flavin adenine dinudeotide (FAD), flavin mononucleotide (FMN) 16 and NADPH and requires the presence of several oxidative cofactors, including tetrahydrobiopterin $\left(\mathrm{BH}_{4}\right),{ }^{17}$ reduced gluthatione, ${ }^{18}$ and a heme complex (Fe) (Figure 1). ${ }^{19}$ Three isoforms of NOS have been documented thus far, based on three different genes that have been cloned and sequenced. There are two isoforms of 


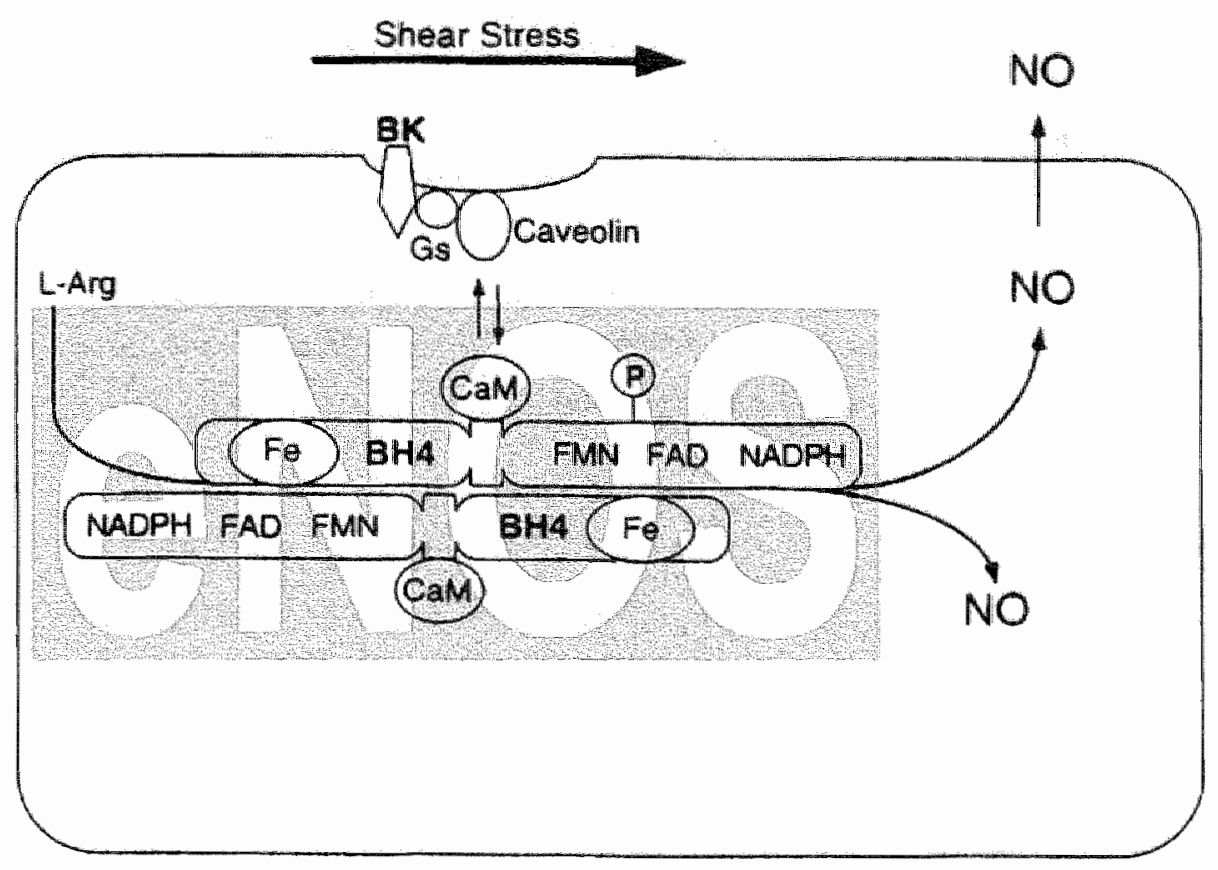

Figure 1. The eNOS enzyme is a homodimer: each monomer has a reductase domain (FAD, FMN and NADPH). The reductase domain supplies electrons to the oxidase domain of the other monomer, where L-arginine is oxidized at the actiwe site, which incorporates a heme group (Fe). Electron rransfer is controlled by the binding of calcium-calmodulin ( $\mathrm{CaM}$ ) to a region between the recluctase and oxidase domains. Tetra hydrobiopterin $\left(\mathrm{BH}_{4}\right)$ is required for homodimerization and modulates the heme redox state during electron transfer. eNOS is activated by agonists that bind specific G protein-coupled receptors ( $\mathrm{eg}$, bradykimin (BK)). These receptors are concentrated in specialized plasma membrane domains, caveolae. Caveolin binds NOS in an inactive sfate. Shear stress activares eNOS by phosphorylation at serine 1179. Adapted from Channon er all ${ }^{272}$

constitutive NOS: eNOS, constiturively expressed in vascular endothelial cells and cardiomyocytes, is membrane-bound and activated only when it binds to calmodulin in a reversible, $\mathrm{Ca}^{2+}$-dependent manner ${ }^{20}$ and nNOS (neuronal NOS), present in brain tissue. The third isoform of NOS is the inducible form (iNOS) and is mainly found in macrophages and neutrophils. ${ }^{21,22}$ It is activated by exposure to bacterial endotoxin or cytokines such as interleukin-1. (IL-1) or $\gamma$-interferon. Inducible NOS is regulated at the transcriptional level and not affected by intracellular $\left[\mathrm{Ca}^{2+}\right] .23$ eNOS is activated by agonists that bind specific $\mathrm{G}$ protein-coupled receptors (eg, bradykinin, acetylcholine, seroronin), leading to elevations in intracellular calcium and activation of calmodulin binding. ${ }^{24,25}$ Caveolin, the major structural protein of caveolae, binds eNOS in an inactive state. Caveolin and calmodulin binding are exclusive; activation releases $\mathrm{NOS}$ from the caveolae. Shear stress activates eNOS by phosphorylation at serine 1179 through a phosphatidylinositol 3-kinase-dependent pathway, independent of calcium-calmodulin. ${ }^{26}$ 


\section{ANGIOPLASTY AS ATREATMENT FOR CORONARY HEART DISEASE}

Atherosclerosis alters the normal structure and function of muscular arteries like the cotonary arterial bed as a consequence of the cellular, mineral, fatty, and connective-tissue components that accumulate within the blood vessel wall. ${ }^{27,28}$ Any reduction of blood flow through an atherosclerotic coronary artery below that required by the oxidative metabolism of working heart muscle (myocardium) establishes the disease state of myocardial ischemia. ${ }^{29}$ If sustained for just a few hours in humans, ischemia may severely compromise cardiac function. This will lead to the formation of an irreversible zone of infarction characterized by dead myocardial tissue. ${ }^{29}$ Immediate reperfusion of ischemic myocardium by reestablishing the coronary blood flow is the onlly well-accepted means for preserving postischemic heart function. ${ }^{30}$ To date, three reperfusion-based interventions have been used for treatment of coronary heart disease: coronary artery bypass grafting, thrombolysis, and percutaneous transluminal coronary angioplasty (PTCA).

$C A B G$ is a major surgical procedure that urilizes a vein (or artery graft) to bypass an atherosclerotic coronary obstruction and route blood from the aorta to the ischemic heart muscle at risk. ${ }^{31}$ The failure rate of venous grafts after $\mathbb{1}$ year approaches $20 \% .^{32}$ The development of neointima or atherosclerosis in the grafted vessel often leads to obliterative stenosis. A quest for less invasive alternatives to CABG supported administration of thrombolytic agents designed to dissolve acure occlusive thrombi, which precede most myocardial infarctions. Unfortunately, tolerance issues, bleeding complications ${ }^{33}$ and ineffective reopening or reocclusion ${ }^{34,35}$ of the artery after reperfusion limit the effectiveness of thrombolysis.

In the most conventional form of PTCA, a collapsed, balloon-ripped catheter is passed across the stenosis under visual guidance. The balloon is then inflated with a fluid contrast solution to disrupt the plaque and enhance both luminal dilation and distal coronary perfusion. ${ }^{36}$ Restenosis still occurs despite advances in treatment i.e. the use of arterial grafts for CABG and stents in percutaneous interventions and is characterized by proliferative and inward remodeling response (see introduction).

\section{RSSTENOSIS AND ITS PATHOBLOLOGY}

Neointima formation is a characteristic response of vessels ro several forms of injury. As such it is one of the processes that contribute to the development of restenosis after a successful balloon angioplasty of a primary stenosis. Restenosis is an occlusive vascular response to trauma characterized by lumen narrowing and is merely a reparative response that has gone on too long or to a greater extent than necessary. Balloon angioplasty stretches the artery wall; in an atherosclerotic vessel this elicirs plaque cracking or splitting and exposes the vessel to overexpansion. Immediate consequences of balloon manipulation on the arterial wall include intimal tearing, endorhelial cell damage, and exposure of subendothelial connective tissue to blood 


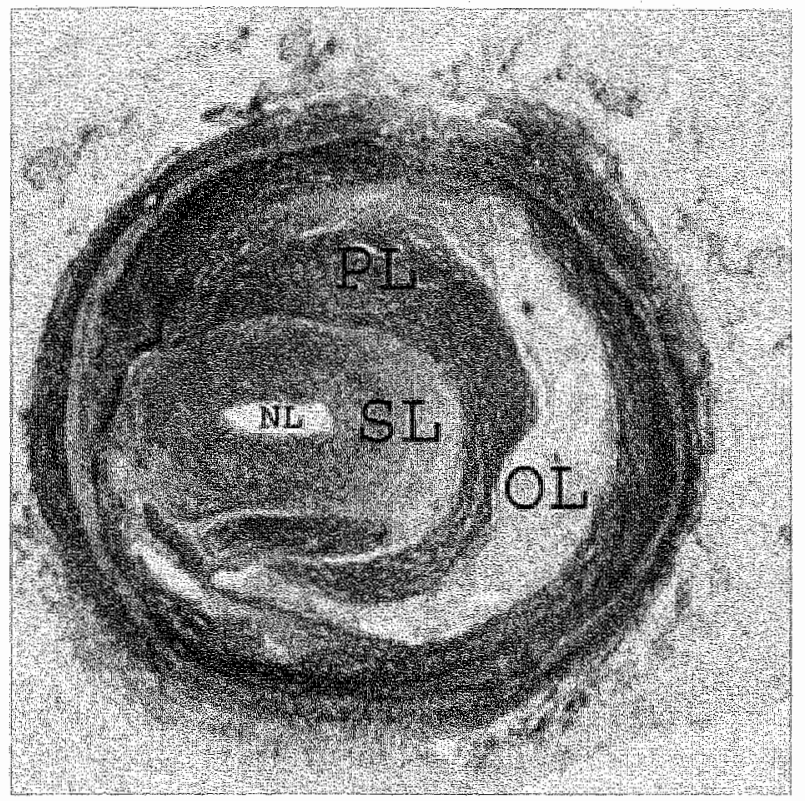

Figure 2. Histologic ontos-section of a typical proliferative lesion in a human coronary artery seweral months. after PTCA. PL, primary lesion; $5 \mathrm{~L}$, secondary lesion; $\mathrm{OL}_{\text {, old lumen; }}$ NL, new lumen. Generously provided by Dr. RJ van Suylen.

components. ${ }^{37-41}$ In response to this trauma, restenosis evolves over several months postangioplasty, usually stabilizing by the sixth month. ${ }^{42}$ Stent implantation has reduced the restenosis rate to approximately $10 \%$ depending on vessel type, lesion location, vessel diameter and patient characteristics. However, since all known angioplasty techniques may damage the blood vessel wall, even the most procedurally successful percutaneous intervention invites restenosis. ${ }^{37,38}$

The pathogenesis of restenosis has been studied most extensively in healthy laboratory animals as the response of normal arteries to mechanical injury. ${ }^{37-40,43-47}$ Complex mechanisms underlying the wound healing response characteristic of restenosis can be identified (Figure 2). These include disruption of the endothelium, platelet activation and thrombus formation, smooth muscle cell migration and proliferation, extracellular matrix changes and remodeling or 'shrinkage' of the artery. Disruption of the endothelium increases surface thrombogenicity, facilitates adhesion of inflammatory cells and results in impaired production of $\mathrm{NO}$ and orher vasodilators that inhibit vasospasm and cellular proliferarion. ${ }^{48}$ Platelet activation and thrombus formation are associated with massive growth factor release from the activated platelets, activation of the clotring cascade with thrombin as a central player, and formation of a thrombus/fibrin matrix which serves as a temporary skeleron for smoorh muscle migration. ${ }^{49,50}$ Vascular smooth muscle cell (VSMC) migration and proliferation are facilitated by the loss of the antimigratory effecr mediated by intact endothelium, growth factor release by platelets and, later on, in an autocrine fashion by smooth muscle cells as well as extracellular matrix degradarion and alteration of its composition. ${ }^{48,51,52}$ Accelerated formation of extracellular matrix is a major contributor of restenosis. Before injury, the smooth muscle cell is 


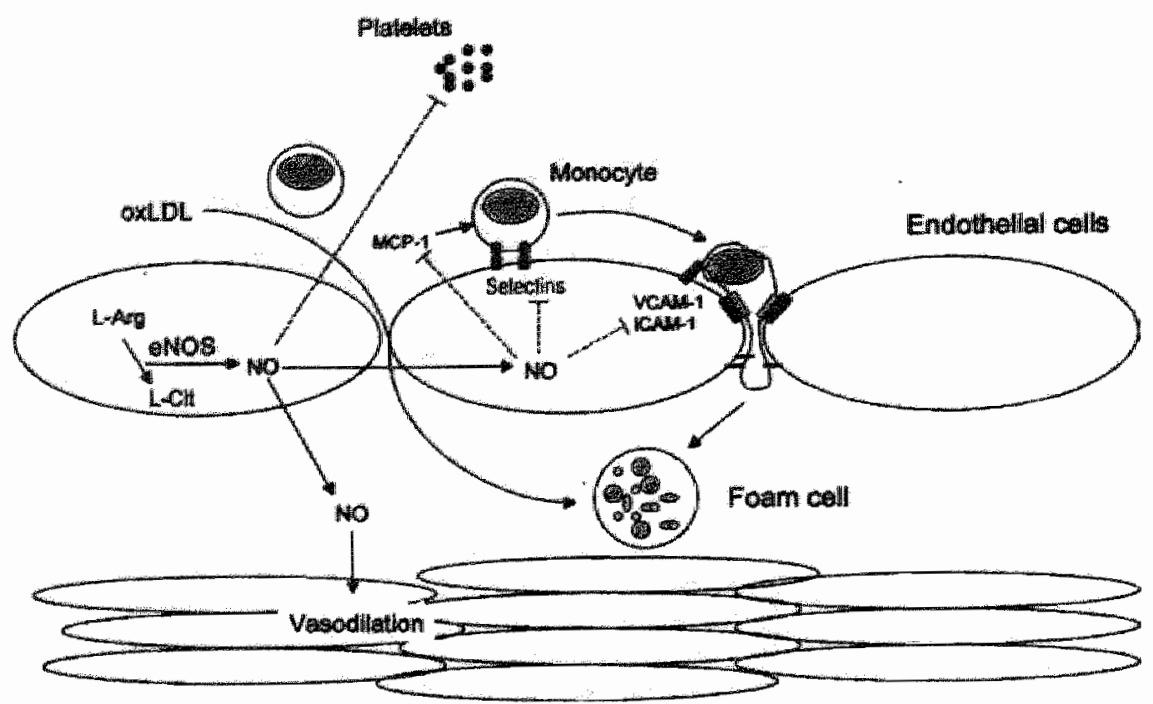

Smooth muscle cells

Figure 3. Diagram indicating NO'santiatheroscleroric effecs in atherosderosis. Dotred lines indicare an inhibitory effect. L-Cir, L-citrulline; ICAM-1, intercellular ad hesion molecule-1. Reprinted from Laufis.

embedded in a dense fibrous extracellular matrix. After injury, this is replaced by fibroproliferative tissue which initially has a loose, myxoid appearance but shows an increase in density over time, associated with an increase in collagen content. ${ }^{53}$ The smooth muscle cell acrively synthesises proteolytic enzymes, glycoproteins and proteoglycans that account for these matrix changes. ${ }^{54}$ In human restenotic lesions, the volume of a typical neointima mainly exists of extracellular matrix, while approximately $10 \%$ of the volume consists of cells. 50,55 Finally, there is evidence that negative remodeling or 'shrinkage' of the artery wirhout an increase in media and intima volume contributes to luminal loss after angioplasty. ${ }^{56}$

\section{NO IN PATHOLOGICAL VASCULAR REMODELING AND CELLULAR PROLIFERATION}

Because restenosis involved both proliferation and inward remodeling $\mathrm{NO}$ is the ideal candidate for therapeutic intervention. NO produced in the endothelium rapidly diffuses to interact with molecular targets in cells in the vascular wall and lumen. ${ }^{2} \mathrm{NO}$ interacts with thiol groups and with metal centers in diverse protein targets, including membrane receptors, $G$ proteins, ion channels, cytosolic enzymes, and transcription factors such as acrivator protein-1 and nuclear factor- $\mathrm{KB}$ (reviewed in reference ${ }^{57}$ ). S-Nitrosylation of thiol groups in plasma proteins such as albumin generates a circularing "pool." of $\mathrm{NO}$-donating groups, ${ }^{58}$ whereas $\mathrm{S}$-nitrosylation of 
hemoglobin in the lung provides nitrosothiol groups to the peripheral vascularure and regulates oxygen delivery. 59,60 In the vascular wall, NO acrivates soluble guanylate cyclase in VSMCs, leading to elevation of CGMP, activation of cGMP-dependent protein kinase ( $\mathrm{PKG}$ ), and vasorelaxation, the primary basis for blood flow and pressure regulation. ${ }^{61}$ In addition to regulating vascular tone, a substantial body of evidence suggests that NO has important antiatherogenic effects ${ }^{62,63}$ (Figure 3). The anti-atherogenic effects of $\mathrm{NO}$ are listed below and are all potentially beneficial in the prevention of restenosis as well.

1. antiplateler effecrs: NO inhibits platelet adhesion and aggregation ${ }^{6}$ and thrombin-induced expression of platelet-activating factor.

2. antiproliferative effects: NO donors potently inhibit VSMC proliferation, ${ }^{64,65}$ migration, ${ }^{66}$ and extracellular matrix synthesis. ${ }^{67}$

3. anti-inflammatory effects: atherogenic stimuli stimulate endothelial expression of adhesion molecules and chemokines, leading to inflammatory cell recruitment. ${ }^{68} \mathrm{NO}$ donors inhibit activation and nuclear translocation of nuclear factor- $\mathrm{KB},{ }^{69,70}$ block cytokine-stimulated endothelial adhesion molecule expression (VCAM-1), ${ }^{70}$ and reduce adhesion and activation of neutrophils and monocytes. ${ }^{71}$ In parricular, inhibition of monocyte chemotactic protein-1 (MCP-1) expression appears to be an important aspect of NO's anti-inflammatory effect in atherosclerosis. ${ }^{72,73}$

4. antioxidant effects: unmodified $\mathrm{LDL}$ undergoes oxidative modifications in the vessel wall that render it highly atherogenic (Figure 3). ${ }^{74}$ Continuous, low-level NO production may directly inhibit lipid oxidation by scavenging free radicals. ${ }^{75}$ From the foregoing, it is clear that $\mathrm{NO}$ is an important determinant of vascular homeostasis. As the vascular response to injury involves many of the above mentioned processes in which $\mathrm{NO}$ acts as a key modularor, NO may also be an unique and potentially powerful candidate to control the remodeling process.

\section{THE ROLE OF HEMODYNAMIC SHEAR STRESS IN ATHEROSCLEROSIS} AND VASCULAR REMODELING

Hemodynamic shear stress, which is the frictional force acting on the endorhelial cell surface as a result of blood flow, has been proposed as a factor regulating blood vessel structure ${ }^{76,77}$ and influencing the development of vascular pathology such as atherosclerosis, ${ }^{78-80}$ aneurysms, ${ }^{81}$ poststenotic dilations, ${ }^{82}$ and arteriovenous malformations. ${ }^{83}$ Despite its associated risk factors, arherosclerosis is a geometrically focal disease that has a propensity to involve the inner edges of blood vessels bifurcations. ${ }^{80,84,85}$ In these susceptible areas, blood. flow is slow, resulting in a low hemodynamic shear stress. In contrast, vessel regions that are exposed to steady blood flow and higher shear stress are less affected. 79, 80, 84-87 Animal, molecular, and cellular studies of the endothelium's response to hemodynamic shear stress have 
provided new insights into its possible contribution to the pathogenesis of atherosclerosis. $88-93$

The luminal surface of the blood vessel and its endothelial surface are constantly exposed to hemodynamic shear stress. ${ }^{83}$ In numerous experiments, shear stress has been shown to actively influence vessel wall remodeling.76,77,94 Changes in blood flow drive both acute and long term compensatory responses that ultimately result in normalization of wall shear stress. In the shorr term, vessels either dilate or constrict, usually due to the local release of wasoactive peptides, to quickly accommodate changes in blood flow. In the case of persistent increases or decreases in flow, however, a different process evolves that includes adlaptive remodeling of the vessel wall, characterized by the reorganization of cellular and extracellular components. This shear stress stabilizing process is dependent on intact endothelial function and is abolished by prior selective destruction of the endothellial monolayer. ${ }^{77}$

An acute increase in blood flow generates a dynamic vascular response resulting in dilation mediated by the endothelial release of $\mathrm{NO}^{1,3}$ prostacyclin ${ }^{65}$ and endothelial derived hyperpolarizing factor ${ }^{96}$ (EDHF) of which the exact nature remains to be determined. A chronic increase in flow causes structural changes involving proliferative responses and matrix degradation. 97,98 These alterations of vascular structure could be prevented by L-NAME ${ }^{98}$ indicating a role for NO. This has further been established in endothelial NO synthase knockout mice which lack compensatory arterial remodeling in response to increased flow. ${ }^{99}$ Elevated shear stress has been shown to increase the production of growth factors like platelet-derived growth factor ${ }^{100}$ (PDGF) and fibroblast growth factor-2101,102 (FGF-2). It has also been shown that NO promotes endogenous FGF-2 production in endothelial cells. ${ }^{103}$ In order to facilitate arterial distensibility and structural remodeling changes in matrix formation must take place. Wong and Langille ${ }^{104}$ demonstrated enlarged fenestrae in the internal elastic lamina (IEL) of flow-loaded rabbit carorid arteries. Matrix metalloproteinases (MMPs) are likely the mediators of IEL degradation in the vessel wall. It has been reported that increased shear stress might stimulate MMP production by endothelial cells and that these cells may secrete factors that trigger MMP production by SMCs. These latter cells are capable of producing MMPs in response to cytokines ${ }^{105}$ or growth factors, like FGF-2. ${ }^{106}$ NO or peroxynitrite directly, ${ }^{107,108}$ or indirectly ${ }^{109}$ via induction of the plasminogen/plasmin system to activate MMPs. Chronic inhibition of NO production by L-NAME prevented MMP-2 activation in flow-loaded carotid arteries and also decreased vascular remodeling, ${ }^{98}$ demonstrating that $\mathrm{NO}$ (or peroxynitrite) is involved in blood flow-induced vascular remodeling. Increased MMP expression and acrivity were associated with the development of neointimal arterial lesions and SMC migration after arterial balloon injury in experimental models, whereas MMP inhibition decreases SMC migration in vitro and in situ. ${ }^{110-113}$ In summary, a large body of evidence suggests an important regulatory role for $\mathrm{NO}$ in the adaptive struc- 
tural changes that occur in the presence of chronic flow increases, both at the level of vascular smooth muscle cell proliferation and extra-cellular matrix degradation.

\section{APRROACHES TO INHIBIT THE VASCULAR RESPONSE TO INIURY USING NO AND NO SURROGATES}

NO gas

Two animals studies revealed that inhalarion of $\mathrm{NO}$ gas inhibited neointimal thickening after vascular damage. 14,115 However, since hemoglobin inactivares $\mathrm{NO}$ with a blood half-life of $-100 \mathrm{~ms},{ }^{16}$ reduction of neointimal formation following $\mathrm{NO}$ inhalation may be a nonspecific response. Furthermore, imhalation of NO for over a few hours may damage mammalian lung rissue.

\section{NOS-related}

L-arginine. The NOS substrate, L-arginine, has antiatherogenic properties in laboratory animals and humans. Supplemental L-arginine restores endothelium-dependent vascular function in hyperlipidemic animalls ${ }^{17-121}$ and humans ${ }^{122-124}$ and inhibits atherosclerosis in rodents. ${ }^{125,} 126$

A large body of evidence reports reduction of neointimal formation by exogenous L-arginine application after vascular injury in healthy and hyperlipidemic rodents. $126-128$

The arginine paradox refers to the discordance between observations made in vitro and those made in vivo regarding the sensitivity of NO synthesis to L-arginine availibility. L-arginine should not be rate-limiting for $N O$ synthesis as the $\mathrm{K}_{\mathrm{tn}}$ of NOS for L-arginine in a cell-free system is in the micromolar range, ${ }^{129}$ while plasma L-arginine concentration is in the 50 micromolar range and endothelial cell $\mathrm{L}$-arginine concentration is in the millimolar range. However, in hypercholesterolemic plasma levels of asymmetric dimethylarginine (ADMA), an endogenous inhibitor of NOS, are elevated. The presence of an endogenous inhibitor in plasma might explain the observation that L-arginine administration in hypercholesterolemic animals and patients results in improved endorhelium-dependent vasodilation. ${ }^{130-132}$

\section{NOS potentiators}

Selected chemical agents and physical stimuli have been reported to potentiate NO synthesis by cytokine-stimulared vascular endothelium and/or smooth muscle. These are a hypoglycemic agent (troglitazone), 133 an antithrombotic drug (ticlopidine), 134 angiotensin converting enzyme (ACE) inhibitors (cilazapril and fosinopril), ${ }^{135}$ a cyclic adenosine monophosphate (CAMP) phosphodiesterase III inhibitor (cilostazol), ${ }^{136}$ a nonselective protein kinase $\mathrm{C}$ inhibitor (staurosporine), ${ }^{137}$ homocysteine, 138 adenosine, ${ }^{139}$ and 8-bromo-cGMP. ${ }^{140}$ 
Two NOS potentiarors relevant to this thesis, estrogen and folare, will be discussed briefly.

The incidence of atherosclerotic disease is low in premenopausal women, rises in postmenopausal women, and is reduced to premenopausal levels in postmenpausal women who receive estrogen therapy. ${ }^{141-143}$ Despite a wealth of supportive observational data, however, the results of randomized clinical trials are mixed. ${ }^{144-146}$ One of the most recent trials showed no cardiovascular benefit but instead a potential for increased harm with a trend towards more rapid angiographic progression of coronary disease in the hormone replacement therapy (HRT) group and a significant increase in death and myocardial infarction in this group. ${ }^{146}$ Another worrisome effect of HRT is the increased risk of breast cancer that is associated with this type of treatment. ${ }^{147}$ Mechanistically, the potentiating effect of estrogen on NO production remains intrigueing and should be persued experimentally, while clinical scenarios and pharmacologic regimens are being developed that should make HRT safe and effective.

Estrogen's protection against atherosclerosis was thought to be related to its effects on serum lipid concentrations, ${ }^{150}$ but this protective effect accounts for only one third of estrogen-induced protection. ${ }^{143,148,149}$ Besides its effect on serum lipid concentration, estrogen alters coagulation and fibrinolytic systems, ${ }^{151-153}$ antioxidant systems, ${ }^{154-159}$ and the production of other vasoactive molecules, ${ }^{160-163}$ such as $\mathrm{NO}$ and prostaglandins, all of which can influence the development of vascular disease.

Reviews of the data suggest that the direct actions of estrogen on blood vessels contributes substantially to the cardiovascular effects of estrogen. $148,149,164$

Estrogen action on blood vessels can be divided into rapid, short-term and longer-term effects.

\section{- Rapid, nongenomic effects}

In normal blood vessels, the endothelium releases $\mathrm{NO}$ in response to various stimuli, which causes vasodilation. ${ }^{165}$ In cell culture studies physiologic concentrations of estrogen activate NOS, which can be blocked by estrogen receptor (ER)- $\alpha$ antagonists. ${ }^{166-168}$ Estrogen rapidly causes coronary vasodilation ex vivo ${ }^{149,164}$ and in vivo in cholesterol-fed ovariectomized primates ${ }^{169}$ and other animals. ${ }^{170}$ Estrogen dilates coronary and brachial arteries in postmenopausal women ${ }^{171-176}$ and, in some studies, in men. ${ }^{175.177,178}$ The short-term coronary vasodilatory effects of estrogen in humans are largely mediated by the increased production of $\mathrm{NO} .176$

-Longerterm offects

Estrogen increases the expression of genes for important vasodilatory enzymes such as prostacyclin synthase and NOS. ${ }^{179,180}$ Estrogen also increased NO bioavailability by increasing the expression of inducible NO synthase gene in endothelium-denuded rat aorta ${ }^{180}$ Long-term administration of estrogen increased acetylcholine-mediated coro- 
nary vasodilation in nonhuman primates, ${ }^{181,182}$ postmenopausal women ${ }^{183}$ and postmenopausal women with angina and normal coronary arteries. 184

\section{- Estrogen and vascular remodeling}

Estrogen facilitates reendothelialization after vascular injury which may be due to increased local expression of vascular endothelial growth factor. 185 Early restoration of endothelial integrity by estrogen may contribute to the atrenuation of the response to injury by increasing the availibility of $\mathrm{NO}$, which can directly inhibit the proliferation of smooth muscle cells. ${ }^{186}$ Estrogen itself has also been shown to directly inhibit the migration and proliferation of smooth muscle cells in vitro. 187,188

Estrogen not only protects against atherosclerotic lesion formation, but it also inhibits the formation of vascular lesions induced by mechanical injury. 149.164. 189-196 The role of vascular estrogen recptors in the protection against mechanical injury was shown in normocholesterolemic mice disrupted of ER- $\alpha$ and ER- $\beta$. In these models, protection was lost against some, but not all, vascular injury endpoints. This raised the possibility that either a third ER exists or that the ER- $\alpha$ was only partially disrupted. The latter seemed to be true; in a newly created mouse with complete disruption of ER- $\alpha$ estrogen no longer protected against wascular injury. 197 The role for ER- $\alpha$ in atheroprotection was confurmed in apoE deficient mice in which the ER- $\alpha$ gene allso had been silenced. 198

\section{- Folates}

The follate tetrahydrobiopterin $\left(\mathrm{BH}_{4}\right)$ is required as a cofactor for NOS activity: all NOS isoforms require tetrahydrobiopterin for NOS homadimerization and for electron transfer during arginine oxidation. ${ }^{199}$ Production of $\mathrm{NO}$ by endothelial cells requires adequate tetrahydrobiopterin levels, ${ }^{200}$ and NOS does not generate NO in the absence of tetrahydrobiopterin; exogenous tetrahydrobiopterin restores enzyme activity 201 More importantly, the enzymatic activity of NOS shifts towards the generation of superoxide rather than $\mathrm{NO}$ when tetrahydrobiopterin is lacking 202 Tetrahydrobiopterin levels are decreased in the diabetic rat aorta and underlic the observed reduction in $\mathrm{NO}$ production. ${ }^{203}$ Exogenous tetrahydrobiopterin partially restores endothelial NO production and reduces NOS-dependent superoxide generation in hypertension, ${ }^{204,205}$ hypercholesterolemia, ${ }^{206}$ and smokers, ${ }^{207}$ whereas inhibition of tetrahydrobiopterin synthesis impairs $\mathrm{NO}$-mediared vasorelaxation. ${ }^{208}$ Thus, tetrahydrobiopterin appears to be an important factor in modulating NOS activity in vascular disease. Furthermore, terrahydrobiopterin levels in atheroscleroctic vessels or in vessels in which the endothelium has been damaged by mechanical injury may augment the beneficial effects of $\mathrm{NO}$ in these sertings. From this it might be clear that endothelial NOS has a dual role in atherosclerosis; under normal conditions it produces low amounts of $\mathrm{NO}$ favoring an antiatherosclerotic milieu. On the other hand, in the diseased state, like hyperlipidemia and arherosclem rosis, it may get uncoupled resulting in more superoxide and less $\mathrm{BH}_{4}$-dependent 
NO production. 209 -methyltetrahydrofolate (5-MTHF), the main form of folate in the circulation, improved endorhelial function that was found to be $\mathrm{BH}_{4}$-dependent ${ }^{210}$ in familial hypercholesterolemia. ${ }^{211}$ Several putative underlying working mechanisms for the effects of 5-MTHF on the enzymatic activity of eNOS have been suggested. 5-MTHF might facilitate electron transfer by $\mathrm{BH}_{4}$ from the reductase domain of eNOS to heme, ${ }^{212}$ enhancing the binding of $\mathrm{BH}_{4}$ to $\mathrm{eNOS}$, increasing $\mathrm{BH}_{4}$ availability via chemical stabilization of $\mathrm{BH}_{4}^{213}$ or stimulate the regeneration $\mathrm{BH}_{4}$ from the inactive oxidized state. ${ }^{214}$

\section{NO donors}

In contrast to $\mathrm{NO}$ donors that generate $\mathrm{NO}$ directly, $215-217$ compounds 218,219 that require either biotransformation or an intermediate to yield NO have been essentially ineffective against arterial injury in normocholesterolemic animals. Recently, beneficial effects of $\mathrm{NO}$-releasing aspirin on postinjury neointimal formation in normo- 220,221 and hypercholesterolemic ${ }^{222-224}$ animals have been published.

NOS gene transfer

Gene transfer vectors allow targeted expression of genes in cells in the vessel wall, provide rools to investigate the function of specific genes in disease processes, and may potentially lead to gene therapy strategies for vascular disease. ${ }^{225}$ For NOS gene transfer, adenoviral vectors have been most widely used, with a smaller number of studies using high-efficiency plasmid-liposome gene delivery and incorporating coat proteins from hemaglutinating virus of Japan. Whereas adenovirus results in the highest efficiency of gene transfer, liposome-mediated gene transfer is a virus-free system with little host immune response and potentially longer transgene expression. Because NO diffuses form NOS-targeted cells to surrounding cells, very high-efficiency transduction may not be an absolute requirement to exerr a biological effect. Experimental studies of NOS gene transfer initially evaluated the expression and activity of recombinant NOS isoforms in cultured cells, ${ }^{226}$ then in isolated vascular rings after ex tivo infection and culture, ${ }^{227-234}$ and finally in several in vivo models of experimental gene therapy. ${ }^{235-248}$ (Table 1) 
Table 1. Sndies of experimental vascular gene therapy with NOS

\begin{tabular}{|c|c|c|c|c|c|}
\hline Vessel: & Species & NOS isoforms & & Vector & End points \\
\hline $\begin{array}{l}\text { Carovid arrery } \\
\text { Adwencitia }\end{array}$ & Rabbit & eNOS & $E x$ tiv? & $\mathrm{Ad}$ & $\begin{array}{l}\text { Restored/augnented } \\
\text { vasorelaxation } 27.28\end{array}$ \\
\hline +Diabetes & Rabbir & eNOS & Exprito & $\mathrm{Ad}$ & Restored vasorelaxation ${ }^{252}$ \\
\hline \multicolumn{5}{|l|}{ *Cholesterol } & Increased vasorelaxation 20 \\
\hline \multirow[t]{2}{*}{ * Ang II hypertension } & Rabbit & NOS & Ex who & Ad & Restored wasorelaxation ${ }^{251}$ \\
\hline & $\begin{array}{l}\text { Mouse } \\
\text { (eNOS-KO) }\end{array}$ & eNOS & Ex wiwo & Ad & Restored wasorclakarion ${ }^{24 n}$ \\
\hline Coronary artery & Pig & eNOS & Exx vito & $\mathrm{Ad}$ & Increased watomoror relaxarion ${ }^{2}$ \\
\hline Basilar artery & Dog & eNOS & Exwivo & $\mathrm{Ad}$ & $\begin{array}{l}\text { Increased } \text { CGMP, increased } \\
\text { vasorclaxation } 2.31 .2 .14\end{array}$ \\
\hline Radial artery & Human & eNOS & Exvino & $\mathrm{Ad}$ & Reduced vasospasm ${ }^{2.32}$ \\
\hline Saphenous vein & Human & eNOS & Ex vivo & $\mathrm{Ad}$ & Recombinant $\operatorname{NOS}^{2,3}$ \\
\hline Carotid artery & Rabbir & nNOS & In wivo & Ad & Increased NO production ${ }^{2 / 18}$ \\
\hline \pm Cholesterol & & eNOS & & & $\begin{array}{l}\text { Reduced endorhelial acrivation and } \\
\text { inflammation }\end{array}$ \\
\hline Adventitia & Rabbit & eNOS & In who & $\mathrm{Ad}$ & $\begin{array}{l}\text { Restored/augmented } \\
\text { vasorelaxation }\end{array}$ \\
\hline \multirow[t]{3}{*}{ *Balloon injury } & Rat & eNOS & In wivo & HVI & $\begin{array}{l}\text { Increased NO production, } \\
\text { increased vasorelaxarion, reduced } \\
\text { intimal liyperplasia }\end{array}$ \\
\hline & Rat & eNOS & In vivo & Ad & $\begin{array}{l}\text { Increased NO production, reduced } \\
\text { intimal hyperplasiats }\end{array}$ \\
\hline & Rat & eNOS & In vivo & Ad & $\begin{array}{l}\text { Reduced intimal hyperplasia, } \\
\text { increased paxillin expression }\end{array}$ \\
\hline+ Hypertension & Rat & eNOS & Yr wino & $\mathrm{Ad}$ & Restored vasorelaxation ${ }^{250}$ \\
\hline Aorta & Rat & NOS & In who & Ad & Reduced intimal hyperplasiat \\
\hline lliac artery & Pig & iNOS & It wito & $\mathrm{Ad}$ & Reduced intimal hyperplasia ${ }^{27}$ \\
\hline $\begin{array}{l}\text { Temoril artery } \\
\text { + Heare Galure }\end{array}$ & Rat & NOS & In wituo & $\mathrm{Ad}$ & Restored vasorelaxarion ${ }^{2}$ dis \\
\hline $\begin{array}{l}\text { Coronary artery } \\
\text { +balloon injury }\end{array}$ & $\operatorname{Pig}$ & eNOS & $\operatorname{In}$ wityo & Ad & $\begin{array}{l}\text { Increased cGMP, redeced innimal } \\
\text { hyperplasia }\end{array}$ \\
\hline $\begin{array}{l}\text { Cercbral arceria via } \\
\text { CSF }\end{array}$ & Dogs & $\operatorname{cNOS}$ & It wito & Ad & $\begin{array}{l}\text { Increased cGMP', increased } \\
\text { wasoretaxation'il }\end{array}$ \\
\hline Aortic allogratt & Rat & iNOS & Shavo & Ad & Reduced allografr arterioseterosis ${ }^{2 / 5}$ \\
\hline \multirow[t]{2}{*}{ Vein grafr } & Dog & cNOS & In wito & HVI & Reduced intima! hyperislasia ${ }^{2}$ atis \\
\hline & Rabbit & nNOS & In wivo & Ad & $\begin{array}{l}\text { Reduced inflammarion, reduced } \\
\text { intimal hyperplasia }{ }^{2 \%}\end{array}$ \\
\hline Pulmonary artery & Rat & eNOS & In wino & Ad & $\begin{array}{l}\text { Increased NO, increased GMP" } \\
\text { reduced thypoxic constriction" }\end{array}$ \\
\hline
\end{tabular}

Ang 11 indicates angiotensin $\mathrm{I}$; $\mathrm{CSF}$, cerebrospinal flaid; $\mathrm{Ad}$, adenowirus; HVJ, hemagglutinating virus of Japan liposomes. 
The ability to restore endothelial vasomotor function in atherosclerotic or mechanically injured arteries represents a critically important test of NOS gene transfer. Ex wivo eNOS gene transfer to isolated vascular rings from normal or hypercholesterolemic rabbit carotid arteries or aorta, ${ }^{229}$ canine basilar arteries, ${ }^{231}$ porcine coronary arteries, ${ }^{230}$ or human saphenous veins ${ }^{233}$ improves vascular relaxations to a variety of vasoactive agonists, such as calcium ionophore, acetylcholine, or UTP. In twwo gene deliwery of eNOS or nNOS confirms that NOS gene transfer can restore or augment regulated $\mathrm{NO}$-mediated vasorelaxation in several sertings, including normal, ${ }^{240}, 242,243$ atherosclerotic, ${ }^{240}$ balloon-injured, ${ }^{236}$ or eNOS-deficient $^{249}$ arteries. Moreover, eNOS gene transfer reduces the vasoconstrictive response to hypoxia in rat lungs ${ }^{247}$ and restores $\mathrm{NO}$-mediated endothelial function in models of cardiac failure, ${ }^{248}$ hypertension ${ }^{250,251}$ or diabetes, ${ }^{252}$ demonstrating that the functional effects of NOS gene transfer are not limited to pharmacological agonist stimulation ex zivo, nor to specific models of endorhelial dysfunction such as hypercholesterolemia.

Exogenous $\mathrm{NO}$, regardless of the route and mode of administration increases intracellular GGMP, inhibit VSMC proliferation, ${ }^{64}$ VSMC migration, ${ }^{66}$ and collagen synthesis, ${ }^{67,253}$ all key components of intimall hyperplasia. Exogenous NO-donating compounds such molsidomine $e^{65}$ or $\mathrm{L}$-arginine ${ }^{254}$ also reduce intimal hyperplasia in vascular injury models in vivo. These results suggest that endorhelial NO may limit VSMC proliferation and neointimal formation in vivo. Gene transfer studies provide direct evidence that local augmentation of vascular NO production by NOS isoforms inhibits intimal hyperplasia in several models of vascular injury: balloon-injured rat ${ }^{236,237,239}$ or pig $^{238}$ arteries, canine venous bypass grafts ${ }^{246}$ and rat aortic allografts 245 and mechanical injured mouse arteries. The effects of $N O$ on cell proliferation and vascular remodeling both contribute to the attenuation of neointima formation in these models. Inhibition of VSMC proliferation, has been demonstrated by reduced incorporation of bromodeoxyuridine and proliferating cell nuclear antigen staining ${ }^{235}$. Changes in VSMC cell phenotype ${ }^{239}$ and cell cycle inhibition have also been observed.255,256 In addition, NOS gene transfer inhibits VSMC migration and matrix remodeling. ${ }^{257}$ Additional mechanisms include increased VSMC apoptosis, ${ }^{258}$ reduced inflammation or plateler activation, ${ }^{259}$ and promotion of endothelial regrowth.

\section{ANIMAL MODELS FOR INTIMAL THICKENING}

In order to unravel the mechanisms of intimal thickening in response to mechanical injury there is a need for appropiate animal models. Intimal thickening occurs in all arteries as a response to a variety of different mechanical traumata, regardless of animal species. Those injuries include such techniques as denudation of endothelial cells by the gentle passage of a balloon embolectomy catheter, the distension of the artery wirh a balloon angioplasty catheter, stent implantation, a rotating wire, ${ }^{260}$ 
photochemical reaction, ${ }^{261,262}$ electrical stimulation, 263 compresston, ${ }^{264}$ by blowing a stream of air through the artery, 265,266 or by a perivascular collar. ${ }^{267-270}$ These strategies may be combined with a high lipid diet to investigate the combined effecr of perturbation of vessel homeosrasis by hyperlipidemia and mechanical injury.

In regard to this thesis the mouse carotid artery denudation model, developed by Lindner, ${ }^{271}$ will be discussed briefly.

\section{Mouse carotid artery denudation model}

In the mouse carotid artery denudation model an intact mouse carotid artery is exposed via a midline incision on the ventral side of the neck. Two ligatures are placed around the external carotid artery, which is tied off with the cranial ligature. The internal carotid artery is temporarily clipped off to prevent back bleeding. A puncture is made between the two ligatures to introduce the denudation guide wire. A curved stainless flexible wire $(0.35 \mathrm{~mm}$ diameter $)$ is introduced via the incision in the external carotid artery into the common carotid artery and under rotation the wire is passed along the vessel three times. The wire is then removed, and the external carotid artery tied off caudal to the incision hole with the caudal ligature. The internal carotid artery is declipped. For intra-arterial applications of drugs a catheter can be introduced via the incision into the external carotid artery. Afrer closure of the skin incision the animals are allowed to recover for several weeks to evaluate several morphometric endpoints.

In this model the passage of the wire removes all endothelial cells from the injured arteries and the denuded surface is covered with a monolayer of platelets adhering to the exposed subendothelial matrix. Maximal replication of medial and inrimal SMC is observed 5 and 8 days after injury and intimal and medial areas are significantly increased two weeks after injury. Complere reendothelialization of the vessel segment (approximately $9 \mathrm{~mm}$ in length) occurs within 3 weeks. ${ }^{271}$

\section{Aim of this thesis}

The aim of this thesis is to investigate the role of $\mathrm{NO}$ in vascular remodeling. The effect of NO on vascular responses to chronically increased flow and mechanical injury are studied in normo- and hyperlipidemic setrings. In addition, the effects of the NOS potentiators estrogen and 5-methyltetrahydrofolate (5-MTHF) are evaluated either as adjuvant therapy to adenoviral eNOS gen transfer or as stand alone interventions (estrogen).

In chapter 2 we use the unilateral carotid artery ligation model in hypercholesterolemic atherosclerotic apoE-deficient mice to assess wherher acute dynamic responses to agonists and flow alterations are predicrive of chronic structural vascular changes in response to increased flow. We characterize agonist induced endorhelial responses in a myograph and acute flow-induced responses in an arteriograph. We hypothesize that in a model of endothelial dysfunction, caused by hypercholesterol- 
emia, acute flow-induced dilations and ourward remodeling to increased shear stress are reduced.

In chapter 3,4 and 5 we use the mouse carotid artery denudation model as a way to evaluate the structural response to mechanical vascular injury. With the use of this mode\ we examine the protective effects and underlying mechanisms of estrogen, a NOS-potentiator, on remodeling after vascular damage in wild type mice and in mice in which either the $\mathrm{NOS}$ gene or the genes encoding both estrogen receptors have been silenced. We hypothesize that estrogen protection is mediated via a NO-dependent pathway and that ER- $\alpha$ and ER- $\beta$ are able to complement one another such that each receptor alone is sufficient to mediate estrogen's protection.

In chapter 6, we use carotid artery injury to remove the endorhelium as a source for eNOS in hyperlipidemic apoE deficient mice with subsequent rescue by local delivery of adenoviral eNOS cDNA alone or in combination with systemic folic acid administration. We hypothesize that restoration of NO production at the site of injury will result in less intimal thickening. Furthermore, we hypothesize that adjunct systemic folic acid delivery will augment the effect of NO on inhibiting neointimal thickening, since 5-MTHF, the active form of folic acid, has been demonstrated to completely restore endothelial function in patients with hypercholesterolemia.

\section{REFERENCES}

1. Furchgot RF, Zawadzki JV. The obligatory role of endothelial cells in the relaxation of arterial smooth touscle by acerylcholine. Narure. 1980;288:373-376.

2. Palmer RM, Ferrige AG, Moncada 5 . Nirric oxide release accounts for the biological activity of endothelium-deriwed relaxing factor. Nature. 1987;327:524-526.

3. Palmer RM. Astuton DS, Moncada S. Vascular endorhelial cells synthesize nitric oxide from Lataginine. Nature. 1988:333:664-666.

4. Ignarro LJ, Buga CM, Wood KS. Byrns RE, Chaudhuri G. Endohelium-derived relaxing factor produced ancl released from artery and vein is nitric oxide. Proc Nat Acad So U S A. 1987;84: $9265-9269$.

5. Huang PL, Huang Z, Mashimo H, Bloch KD, Moskowitz MA, Bevan JA, Fishman MC. Hyperrension in mice lacking the gene for endothelial nitric oxide synthase [see comments]. Nature. $1995,377: 239-242$.

6. Radomski MW, Palmer RM, Moncada $S$. The role of nitric oxide and CGMP in platelet adhesion to vascular endothelium. Bioskm Biophys Res Commun. 1987;148:1482-1489.

7. Toda N, Okamura T. Modification by L-NG-monomethyl arginine (L-NMMA) of the response to nerve stimulation in isolated dog mesenteric and cerebral arteries. Ipn / Phamacol. $1990,52: 170-173$

8. Toda N, Okamura T. Possible role of nitric oxide in transmitring information from vasodilator nerve to cerebroarterial muscle. Biochem Brophys Res Commus. 1990;170:308-313. 
9. Moncada S. The 1991 Ulf won Euler Lecrure. The L-arginine: nutric oxide pathway Anta Plyant Scand. 1992; $145: 201-227$.

10. Moncada S, Palmer RM, Higgs EA. Nitric oxide: physiology parhophysiology, and pharmacology. Pharmacol Rev. 1991;43:109-142.

11. Stamler $\int S$, Singel DJ, Loscalzo J. Biochemistry of nitric oxide and its redox-acrivated forms. Science. 1992;258:1898-1902.

12. Archer 5 . Measurement of nitric oxide in biological models. Fode f. 19937349-360.

13. Wennmalm $A$, Benthin $G$, Petersson AS. Dependence of the metabolism of nitric oxide (NO) in healthy human whote blood on the oxygenation of its red cell haemoglobin. Br. I Pformatol. 1992:106:507-508.

1.4. Lancaster JR, Jr. Simulation of the diffusion and reaction of endogenously produced nitric oxide. Proc Natl Acad Sc: US A. 1994;91:8137-8141.

15. Nathan C. Nitric oxide as a secrerory product of mammalian cells. Fateb /, 1.992:6:305:1-3064.

16. Bredt DS, Ferris CD, Snyder SH. Nitric oxide synthase regulatory sites. Phosphorylation by cyclic AMP-dependent protein kinase, protein kinase $\mathrm{C}$, and calcium/calmodulin prorein kinase identification of flawin and calmodulin binding sites. J Biol Chem. 1992;267:10976-10981.

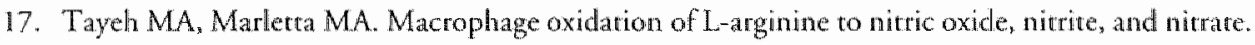
Terrahydrobiopterin is required as a cofactor. / Brol Chem. 1989;264:19654-19658.

18. Suehr DJ, Kwon NS, Nathan CF. FAD and GSH participate in macrophage synthesis of nitric oxide. Biochem Bropbys Res Comman. 1990;168:558-565.

19. White KA. Marletta MA. Nitric oxide synthase is a cytochrome P-450 rype hemoprocein. Bio chemistry. 1992;31:6627-6631.

20. Busse $\mathrm{R}$, Fleming I. Regulation and functional consequences of endothelial nitric oxide formation. Ann Med. 1995;27:331-340.

21. Marletra MA, Yoon PS, Iyengar R, Leaf CD, Wishnok JS. Macrophage oxidation of L-arginine to nitrite and nitrate: nitric oxide is an intermediate. Biochemistry. 1988;27:8706-8711.

22. Yui $Y$, Hatron $R_{\text {, Kosuga } K}$, Eizawa H, Hiki K, Ohkawa $S$, Ohnishi K, Terao $S$, Kawa $\mathbb{C}$. Calmodulin-independent vitric oxide synthase from rat polymorphonuctear neurrophils. I Biol Chem 1991:266:3369-3371.

23. Xie QW, Cho HI, Calaycay I, Mumfond RA, Swiderek KM, Lee TD, Ding A, Troso T, Marhan C. Cloning and characterization of inducible nirric oxide synthase from mouse macrophages. Sciente. $1992,256: 225-228$.

24. Bredr DS, Snyder SH. Isolarion of nitric oxide synohetase, a calmodulin-requiring enzyme. Proc Natl Acal Sci U S A. 1990;87:682-685.

25. Busse R, Mulsch A. Calcium-dependent nitric oxide synthesis in endothelial cytosol is mediated by calmodulin. FEBS Lett. 1990;265:133-136.

26. Lamontagne D, Pohl U, Busse R. Mechanical deformation of vespel wall and shear stress deternine the basal release of endothelium-derived relaxing factor in the intact rabbit coronary vascular bed. Cin Res. 1992;70:123-130.

27. Janero DR. Therapeutic potential of vitamin E in the pathogenesis of spontaneous arherosclerosis. Free Radic Ból Med. 1991:11:129-144. 
28. Vogel RA. Coronaty risk factors, endothclial function, and atherosclerosis: a review. Clin Cardiod. $1997: 20: 426-432$.

29. Opic LH. Myocardial ischemiamerabolic pathways and implications of increased glycolysis. Cardiowas Drugs Ther. 1990;4:777-790.

30. Berger PB, Ellis $\$$, Holmes DR, Jr., Granger CB. Criger DA, Berriu A, Topol El, Califf RM. Relationship beween delay in performing direct cononary angioplasty and early clinical ontcome in patients with acute myocardial infarction: results from the global use of strategies to open occluded artaries in Acure Coronary Syndromes (GUSTO-IIb) trial. Circulation. 1999;100:14-20.

31. Kaiser GC. CABG 1984: technical aspects of bypass surgery. Circulation. 1985:72:V46-58.

32. Zou Y, Dietrich H, Hu Y, Metzler B, Wick G, Xu Q. Mouse Model of Venous Bypass Graft Arteriosclerosis. Anat / Patbol: 1998;153:1301-1310.

33. Antman EM. Hirudin in acute myocardial infarction. Thrombolysis and Thrombin Inhibition in Myocardial Infarction (TIM1) 9B triall. Cimculation. 1996;94:911-921.

34. Anderson \L, Karagounis LA, Becker LC, Sorensen SG, Menlowe RL. TIMI perfusion grade 3 bur not grade 2 resuls in improved ourcome after chrombolysis for myocardial infarction. Ventriculographic, enzymatic, and electrocardiographic evidence from the TEAM-3 Study. CiruLation. 1993:87:1829-1839.

35. Anderson JL. Karagounis LA, Califf RM. Metaanalysis of five reporred studies on the relation of early coronary patency grades with mortaliny and outcomes after acute myocardial infarction. Am $J$ Cardiol $1996 ; 78: 1-8$.

36. Konowski R, Mehran $\mathbb{R}_{x}$ Hong MK, Sader LF, Pichard AD, Kent KM, Mintz GS, Waksman R, Laird JR, Lansky AJ, Bucher TA, Popma JJ, Leon MB. Procedural results and late dinical outcomes after placement of three or more stents in single coronary lesions. Circulation. 1998; 97: 1355-1361.

37. Bauters C, Isner JM. The biology of restenosis. Prog Cardiowase Dis. 1997:40:107-11.16.

38. Casterella PJ, Teirstein PS. Prevention of coronary resrenosis. Cardiol Rey. 1999;7:219-231.

39. Waller BF. Pathology of transluminal balloon angioplasty used in the treatment of coronary hatr diserse. Hum Pabol, 1987:18:476-484.

40. Gravanis MB, Roubin GS. Histopathologic phenomena at the site of percutaneous rransluminal Cononary angioplasty; the problen of rescenoxis. Hum Patbol. 1989;20:477-485.

41. Potkin BN. Robers WC. Effects of percutancous transluminal coronary angioplasty on atherosclerotic plaques and relation of plaque composition and arterial size to outcome. $A m J$ Cardiol 1988:62:41-50.

42. Fleisch M. Meier B. Management and outcome of stents in 1998: long-rerm outcome. Cardiol Rew 1999;7:215-218.

43. Lefkovirs J. Topol EJ. Pharmacological approaches for the prevention of restenosis after percutaneous coronary intervention. Prog Cardiovasi Dis. 1997:40:141-158.

44. Bauters C. Meurice T, Hamon M, McFadden E, Lablanche JM, Bertrand ME. Mechanisms and prevention of restenosis: from experimental models to clinical practice. Cardiovase Res. 1996;3:1: $835-846$.

45. Mak KH, Topol EJ. Clinical rrialls to prevent restenosis after percutaneows coronary revascularization. Ann N Y Acad Sci 1997;811:255-284; discussion 284-258. 
46. Gallo $R$, Padurean A, Toschi V, Bichler J. Fallon JT, Chesebro JH, Fuster V, Badimon JJ. Prolonged thrombin inhibirion reduces restenosis after balloon angioplasty in porcine coronary arteries. Circulation. 1998;97:581-588.

47. Lafont $A$, Faxon $D$. Why do animal models of post-angioplasty restenosis sometimes poorly predict the ourcome of clinical trials? Candionas Res. 1998;39:50-59.

48. Cohen RA. The role of nirric oxide and orher endorhelium-derived wasoactive substances in vascular disease. Prog Cardiovasc Dis. 1995;38:105-128.

49. Casscells W. Snooth muscle cell growth factors. Prog Growth Factor Res. 1991:3:177-206.

50. Schwart RS, Holmes DR, Jr., Topol EJ. The restenosis paradigm revisited: an alternative proposal for cellular mechanisms. J Am Coll Cardiol 1992;20:1284-1293.

51. Miano JM, Vlasic $\mathbb{N}$, Tota RR, Scemerman MB. Smooth muscle cell immediate-early gene and growth factor activation follows vascular injury. A purative in wivo mechanism for autocrine growty. Anteriascer Thromb. 1993;13:211-219.

52. Jackson CL, Raines EW, Ross R, Reidy MA. Role of endogenous plateler-derived growth factor in arterial smooth muscle cell migration after balloon catherer injury. Arteriosder Thromb. $1993 ; 13: 1218-1226$.

53. Strauss BH, Umans VA, van Suyten RJ, de Feyter PJ, Marco J, Robernson GC, Renkin J, Heyndrickx $G$, Vuzevski VD, Bosman FT, et al. Directional atherecromy for treatment of restenosis within coronary stents: dinical, angiographic and hiscologic results. I Am Coll Cardiol. $1992 ; 20: 1465-1473$

54. Coats WD, Jr., Whittaker P, Cheung DT, Currier JW, Han B, Faxon DP. Collagen content is significandy lower in restenotic versus nonrestenocic vessels after balloon angioplasty in the atherosclerotic rabbit model. Circulation. 1997:95:1293-1300.

55. Nobuyoshi M, Kimura T, Ohishi H, Horiuchi H, Nosaka H, Hamasaki N, Yokoi H, Kim K. Restenosis after percutaneous transluminal coronary angioplasty: pathologic observations in 20 parients. Am Coll Cardiol. 1991;17:433-439.

56. Kimura T, Kaburagi S, Tanura T, Yokoi H, Nakagawa Y, Hamasaki N, Nosaka H, Nobuyoshi M, Mintz GS, Popma J, Leon MB. Remodeling of human coronary arteries undergoing coronary angioplasty or atherectomy. Circulution. 1997996:475-483.

57. Samler 15 . Redox signaling: nitrosylation and related ratget interacrions of nitric oxide. Cell. $1994: 78: 931-936$

58. Keaney JF, Jr, Simon DI, Stamler JS, Jaraki O, Scharfstein J. Vita JA, Loscalzo J. NO forms an adducr with senum albumin that has endothelum-derived relaxing factor-like properries. $/ \mathrm{Clin} / \mathrm{n}$ vest. 1993:21:1582-1589.

59. Jia L, Bonawentura C, Bonaventura J. Stamler JS. S-nitrosohamoglobin: a dynamic activity of blood involved in vascular control. Nature. 1996;380:221-226.

60. Gow AJ, Luchsinger BP. Pawloski JR. Singel DJ, Stamler JS. The oxyhemoglobin reaction of nirric oxide. Proc Navl Acad Sa U S A. 1999:96:9027-9032.

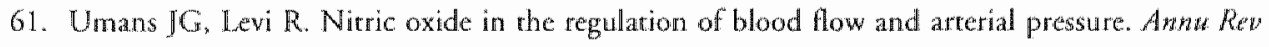
Physiol 1995:57:771-790. 
62. Harrison $D G$, Ohata $Y$. Physiologic conseguences of increased vascular oxidant stresses in hypercholesterolemia and atherosclerosis: implications for impaired vasomotion. Am $\mathrm{J}$ Cardiol. $1995 ; 75,75 \mathrm{~B}-81 \mathrm{~B}$.

63. Lloyd-Jones $\mathrm{DM}$, Bloch $\mathrm{KD}$. The vascular biology of nitric oxide and its rolle in acherogenesis. Anns Rep Mod 1996:47:365-375.

64. Garg UC, Hassid A. Niric axide-generating vasodilators and 8-bromo-cyclic guanosine monophosphate inhibit mitogenesis and proliferation of cultured rar vascular smooth muscle cells. J Clin Invest. 1989;83:1774-1777.

65. Growes PH, Banning AP, Penny W, Newby AC, Cheadle HA, Lewis MJ. The effects of exogenous nitric oxide on smooth muscle cell proliferarion following porcine carotid angioplasty. Cardiowasc Res. 1995;30:87-96.

66. Sarkar R, Meinberg EG, Stanley JC, Gordon D, Webb RC. Nitric oxide reversibly inhibits the migration of cultured wascular smooth muscle cells. Cinc Res. 1996;78:225-230.

67. Kolpakov V, Gordon D. Kulik TJ. Nitric oxide-generaring compounds inhibit rotal protein and collagen synthesis in cultured vascular smooth muscle cells. Circ Res. 1995:76:305-309.

68. Navab M, Hama SY, Nguyen TB, Fogelman AM. Monocyte adhesion and transmigration in athcrosclerosis. Coran Artery Dis, 1994;5:198-204.

69. Peng HB, Libby $\mathrm{J}^{3}$, Liao JK. Induction and stabilization of I kappa B alpha by nitric oxide mediates inhibition of NF-kappa B. J Biol Chem. 1995;270:14214-14219.

70. De Caterina R, Libby P, Peng HB. Thannickal VJ, Rajavashisth TB, Gimbrone MA, Jr., Shin WS, Liao JK. Nitric oxide decreases cytokine-induced endotheliat activation. Nitric oxide selectively reduces endothelial expression of adhesion molecules and proinflammatory cytokines. I Clin Inwext. 1995:96:60-68.

71. Gauthier TW, Scalia R, Murohara T, Guo JP, Lefer AM. Nitric oxide protects against leukocyce-endorhelium inceractions in the early stages of hypercholesterolemia. Anterrorder Thromb Vasc Biol. 1995:15:1652-1659.

72. Zeiher AM, Fisslthaler B, Schray-Utz B, Busse R. Nitric oxide modulates the expression of monocyte chemoatractant protein 1 in cultured human endothelial cells. Cire Res. 1995:76:980-986.

73. Tsao PS, Wang B, Buitrago R, Shyy JY, Cooke JP. Nitric oxide regulates monocyte dhemotacric protcin-1. Circulation. 1997;96:934-940.

74. Heinecke JW. Mechanisms of oxidarive danage of low density lipoprotein in human atherosclerosis. Curr Opin Lipudtol 1997:8:268-274.

75. Hogg N, Kalyanaraman B, Joseph ل, Struck A. Parthasararhy S. Inhibition of low-density lipoprotein oxidation by nirric oxide. Potential role in atherogenesis. $Y E B S$ Lett. 1993;334:170-174.

76. Kamiya $A$, Togawa $T$. Adaprive regulation of wall shear stress to flow change in the canine carotid artery. Am Pbysal 1980;239:H14-21.

77. Langille BL, O Donnell F. Reductions in arterial diameter produced by chronic decreases in blood How are endothelium-dependent. Science. 1986;231:405-407.

78. Fry DL. Certain histological and chemical responses of the vascular interface to acurely induced mechanical stress in the aorta of the dog. Cinc Res. 1969;24:93-108. 
79. Caro CG, Fitz-Gerald JM, Schroter RC. Atheroma and arteriall wall shear. Observation, correlition and proposal of a shear dependent mass ransfer mechinism for atherogenesis. Pror R Sor Lond B Biol Sci. 1971;177:109-159.

80. Zarins CK, Giddens DP, Bharadvaj BK, Sotriurai VS, Mabon RF, Glagov S. Carotid bifurcation atherosclerosis. Quantitarve correlarion of plaque localization with flow velocity profies and wall shear stress. Cinc Res. 1983;53:502-514.

81. Kerber CW, Hecht ST, Knox K, Buxton RB, Meltzer HS. Flow dynamics in a fatal aneurysm of the basilar artery. ANR Am / Newrowdiol. 1996;17:1417-1421.

82. Rodbard S. Vascular caliber. Cardiolagy 1975;60:4-49.

83. Rossirri S, Svendsen P. Shear stress in cerebral arteries supplying arteriovenous malformations. Acta Nearochir (Wien) 1995:137:138-145.

84. Asakura T, Karino T. Flow patterns and sparial distriburion of atherosclerotic lesions in human coronary arteries. Circ Res. 1990;66:1045-1066.

85. Gnasso A, Irace C, Carallo C, De Franceschi MS, Motri C, Matrioli PL, Pujia A. In vivo associarion between low wall shear stress and plaque in subjecrs with asymmetrical carorid atherosclerosis. Stroke. 1997;28:993-998.

86. Motoniya M, Kamino T. Flow patterns in the human carotid artery bifurcation. Stroke. 1984;15:50-56.

87. Bharadvaj BK, Mabon RF, Giddens DP. Steady flow in a model of the human carotid bifurcation. Pant 1I-laser-Doppler anemometer measurements. J Biomech. 1982;15:363-378.

88. Davies PF. Flow-mediared endorhelial mechanomansducrion. Physio/ Rev. 1995:75:519-560.

89. Malek AM, Izumo S. Control of endothelial cell gene expression by flow. J Bionech. $1995 ; 28: 1515-1528$.

90. Gimbrone MA, Jr. Vascular endothelium: an integrator of pathophysiologic scimuli in arherosclerosis. Am J Cardial. 1995:75:67B-70B.

91. Nerem RM, Alexander RW, Chappell DC, Medford RM, Varner SE, Taylor WR. The study of the influence of flow on wascular endorhelial biology, Am J Mod Sci. 1998;316:169-175.

92. Chien S, Li S, Shyy M. Effects of mechanical forces on signal rransduction and gene expression in endorhelial cells. Hypertusion. 1998:31:162-169.

93. Traub $O$, Berk $B C$. Laminar shear stress: mechanisms by which endothelial cells transduce an atheroprotectiwe force. Arteriaster Throwb Vasc Brol 1998;18:677-685.

94. Kraiss LW, Kirkman TR, Kohler TR, Zierler B. Clowes AW, Shear stress regulates smooth muscle proliferation and neoincimal thickening in porous polyterafleorocthylene grafts. Arterioscler Thromb. 1991;11:1844-1852.

95. Berthiame F, Frangos JA. Flow-induced prostacyclin producrion is mediated by a pertussis toxin-sensitive G protein. FEBS Lets. 1992;308:277-279.

96. Huang A, Sun D, Carroll MA, Jiang H, Smith CJ, Connetta JA, Falck JK, Shesely EG, Koller A, Kaley $\mathrm{G}$. EDHF mediates flow-induced dibtion in skelectal muscle arterioles of female eNOS-KO mice. Am J Physiol Heant Circ 19ysiol. 2001;280:H2462-2469.

97. Lehman RM, Owens GK, Kassell NF, Honga K. Mechanism of enlargenent of major cerebral collareral arteries in rabbits. Stroke. 1991:22:499-504. 
98. Trone F, Wassef M, Esposito B. Henrion D, Glagov S, Tedgru A. Rolle of NO in flow-induced remodeling of the rabbir common carovid artery. Arterioster Thromb Vase Biol. 1996;16: $1256-1262$.

99. Rudic RO, Shesely EG, Maeda N, Smithies O, Segal SS, Sessa WC. Direce evidence for the imporm tance of endothelium-derived nitric oxide in vascular remodeling. I Clin Invest. 1998;101:731-736.

1.00. Hsich HJ, Li NQ, Frangos JA. Shear-induced platelet-derived growth facror gene expression in human endothelial cells is mediated by protein kinase C. J Cell Physol 1.992;150:552-558.

101. Malek AM, Gibbons GH, Dzau VJ, Izumo S. Fluid shear stress differentially modulates expression of genes encoding basic fibroblast growth factor and platelet-derived growth factor $B$ chain in vascular endothelium. J Clin Iwwest. 1993;92:2013-2021.

102. Singh TM, Abe KY, Sasaki T, 2huang YJ, Masuda H, Zarins CK. Basic fibroblast growth factor expression precedes flow-induced arterial enlargement. I Sturg Res. 1998;77:165-173.

103. Ziche M, Parenti A, Ledda F, Dell'Era P, Granger HJ, Maggi CA, Presta M. Nitric oxide promotes proliferation and plasminogen activator production by coronary venular endothelium through endogenous bFGF. Crre Res. 1997;80:845-852.

104. Wong LC, Langille BL. Developmental remodeling of the internal elastic lamina of rabbit arteries: effect of blood flow. Circ Res. 1996;78:799-805.

105. Galis ZS. Muszynski M, Sukhova GK, Simon-Morrissey E, Unemori EN, Lark MW, Amento E, Libby $\mathrm{P}$. Cytokine-stimulated human wascular smooth muscle cells synthesize a complement of enzymes required for extracellular marrix digestion. Carc Res. 1994;75:181-189.

106. Pickering J $\mathrm{G}$, Ford $\mathrm{CM}$, Tang B, Chow I.H. Coordinated effects of fibroblast growth factor-2 on expression of fibrillar collagens, matrix metalloproteinases, and cissue inhibitors of matrix metalloproteinases by human vascular smooth muscle cells. Evidence for repressed collagen production and activated degradative capacity. Anterioscler Thromb Vas Biol. 1997; 17:475-482.

107. Murrell GA, Jang D, Williams RJ. Nitric oxide acrivates metalloprotease enzymes in articular cartilage. Biochem Biophys Rer Commun. 1995;206:15-21.

108. Rajagopalan S. Meng XP, Ramasamy S, Harrison DG, Galis ZS. Reactive axygen species produced by macrophage derived foam cells regulate the acriviry of vascular matrix metalloproteinases in vitro. Implications for atherosclerotic plaque stability. J Clin Inwest. 1996;98:2572-2579.

109. Carmeliet P. Moons L, Lijnen R, Baes M, Lemaitre V, Tipping P. Drew A, Eeckhout Y, Shapiro S, Lupu IF, Collen D. Urokinase-generated plasmin activates matrix metalloproteinases during aneurysm formarion. Nat Genet. 1997:17:439-444

110. Southgare KM, Fisher M, Barning AP, "Thurston VJ, Baker AH, Fabunmi RP, Groves PH, Davies M, Newby AC. Upregulation of basement membrane-degrading metalloproteinase secrecion after ballown injury of pig caronid arteries. Circ Res. 1996;79:1177-1187.

111. Bendeck MP, Zempo N, Clowes AW, Galardy RE, Reidy MA. Smooth muscle cell migration and matrix mealloproteinase expression after arterial injury in the rat. Circ Res. 1994;75:539-545.

112. Zempo N, Kenagy RD, Au YP, Bendeck M, Clowes MM, Reidy MA, Clowes AW. Matrix metalloproteinases of vascular wall cells are increased in balloon-injured rar carotid artery $/$ Vaac
Sung. 1994;20:209-217. 
113. Forough R, Koyama N, Hasenstab D, Lea H, Clowes M, Nikkari ST, Clowes AW. Overexpression of tissue inhibiror of matrix metalloproremase-I inhibirs vascular smooth muscle cell functions in vitro and in wivo. Cirt Res. 1996;79:812-820.

114. Lee JS, Adrie C, Jacob HJ, Robers JD, Ir., Zapol WM, Bloch KD. Chronic inhalation of nitric oxide inhibits neointimal formation after balloon-indnced arrerial injury. Cire Res. 1996;78:337-342.

115. Adrie C, Bloch KD, Moreno PR, Hurtord WE, Guerrero JL, Holt R, Zapol WM, Gold HK. Semigran MJ. Inhaled nitric oxide increases coronary artery patency after thrombolysis. Conculantron. 1996;94:1919-1926.

116. Kelm M, Schrader J. Control of coronary wascular tone by nitric oxide. Cine Res. 1990;66: $1561-1575$.

117. Cooke IP, Andon NA, Girerd X], Hirsch AT, Creager MA. Arginine restores cholinergic relaxation of hypercholesterolemic mabit thoracic aorta. Criculation. 1991;83:1057-1062.

118. Girerd XJ. Hirsch AT, Cooke JP, Dzau VJ, Creager MA. L-arginine augments endothelium-dependent vasodilation in cholesterol-fed rabbits. Circ Res. 1990;67:1301-1308.

1 19. Rossitch E, Jr., Alexander E, 3rd, Black PM, Cooke JP. L-arginine normalizes endorhelial function in cerebral vessels from hypercholesterolemic rabbirs. / Clin Invest. 1991;87:1295-1299.

120. Singer AH, Tsao PS, Wang BY, Bloch DA, Cooke JP. Discordant effects of dietary L-arginine on vascular seructure and reacrivity in hypercholesterolemic rabbits. / Cardiovase Pbarmatod. $1995 ; 25: 710-716$.

121. Cooke JP, Singer AH, Tsao P, Zera P, Rowan RA, Billingham ME. Antiatherogenic effects of L-arginine in the hypercholesterolemic rabbit. J Clin Invest. 1992;90:1168-1172.

122. Lerman A, Bumett JC, Jr., Higano ST, McKinley LJ, Holmes DR, Jr. Long-term L-arginine supplementation improves small-vessel coronary endothelial function in humans. Circhlation. 1998;97:2123-2128.

123. Drexler $\mathrm{H}$, Zeiher AM, Meinzer $\mathrm{K}$, Just H. Correction of endothelial dysfunction in coronary microcirculation of hypercholesterolacmic patients by Larginine. Lancet. 1991;338:1546-1550.

124. Dubois-Rande JL, Zelinsky R, Roudot F. Chabrier PE, Castaigne A, Geschwind H. Adnot S. EFfects of in fusion of 1 -arginine into the left anterior descending coronary artery on acerylcholine in. duced wasoconstriction of human atheromatous coronary arteries. Am I Catiols 1992;70:1269-1275.

125. Boger RH, Bode-Boger SM. Brandes RP, Phivthong-ngam L, Bolime M, Nafe R, Mugge A. Frolich JC Dietary L-arginine reduces the progression of atherosclerosis in cholesterol-fed rabbita: comparison with lovastatin. Circulation. 1997;96:1282-1290.

126. Wang BY, Candipan RC, Arjomandi M, Hsiun PT, Tsao PS, Cooke JP Arginine restores nitric oxide acriviry and inhibirs monocyte accumulation after vascular injury in hypercholesterolemic rabbits. J Am Coll Cardiol. 1996;28:1573-1579.

127. McNamara DB, Bedi B, Aurora H, Tena L, Ignarro LI, Kadowitz PJ, Akers DL. Larginine inhibirs balloon catherer-induced incimal hyperplasia. Biochem Biophys Res Cownun. 1993;193: $291-296$.

128. Taguchi ], Abe J, Okazaki $H$, Takuwa $Y$, Kurokawa $K$. L-arginine inhibits neoinrimal formation following balloon injury. Life Sri. 1993:53:PL387-392. 
129. Pollock JS, Forstermann U, Mitchell IA, Warner TD, Schmidr HH, Nakane M, Murad F. Purifcation and characterization of particulate endothdium-derived relaxing factor synthase from cultured and native bovine aortic endothelial cells. Proc Nat Acad So U S.A. 1991:88:10480-10484.

130. Boger RH, Bode-Boger SM, Sxuba A, Tsao PS, Chan JR, Tangphao O, Blaschke TF, Cooke JP. Asymmetric dimethylarginine (ADMA): a novel risk facror for endothellal dysfunction: ats role in hypercholesterclenia. Circulation. 1998;98:1842-1847.

131.Yu X], Li Y, Xiong Y. Increase of an endogenous inhibitor of nitric oxide synthesis in serum of high cholesteroll fed rabbits. Life Sei. 1994;54:753-758.

132. Bode-Boger SM, Boger RH, Kienke S, Junker W, Frolich JC. Elevated L-arginineldimethylarginine ratio contributes to enhanced systemic NO production by dietary I-arginine in hypercholesterolemic rabbits. Biochem Biophys Res Commun. 1996;219:598-603.

133. Hatcori $X$, Hatron $\$$, Kasai $K$. Troglitazone upregulares nitric oxide synthesis in wascullar smooth muscle cells. Hypertension. 1999;33:943-948.

134. Di Minno G, Cerbone AM. Mattioli PL, Turco S, Lovine C, Mancini M. Functionally thrombasthenic state in normal platelets following the administration of riclopidine. / Clin Invest. $1985,75: 328-338$.

135. Frollich ED. Angioremsin corverting enzyme inhibitors. Present and furure. Hypertension. $1989 ; 13: 1125-130$.

136. Ikeda U, Ikeda M, Kano S, Kanbe T, Shimada K. Effect of cilostazol, a cAMP" phosphodiesterase inhibitor, on nitric oxide production by vascular smooth muscle cells. Esur J Pharmatol. 1996:314:197-202.

137. Hecker M, Preiss C, Schini-Kerth VB. Induction by staurosporine of nitric oxide syuthase expression in vascular smooth muscle cells: role of $\mathrm{NF}$-kappa $\mathrm{B}, \mathrm{CREB}$ and C/EBP beta. Br J Phatmacol. 1997:120:1067-1074.

138. Ikeda U, Ilkeda M, Minota S, Shimada K. Homocysteine increases nitric oxide synthesis in grokine-stimulated wascular smooth muscle cells. Circulation. 1999,99:1230-1235.

139. Ikeda U, Kurosaki Ko. Ohya K, Shimada K. Adenosine stimulates nitric oxide synchesis in vascular smooth muscle cells. Cardiowisc Res. 1997;35:168-174.

1.40. Inoue T, Fukuo K, Nakahashi T, Hata S, Morimoto S, Ogithara T. cGMP upregulates nitric oxide synthase expression in wascular smoon muscle cells. Hypertension. 1995;25:7 11-714.

141. Barret-Connor E. Sex Differences in Coronary Heart Disease: Why Are Women So Superior? The 1995 Ancel Keys Lecture. Cinalation. 1997;95:252-264.

142. Stampfer MJ, Coldiz GA, Willett WC, Manson JE, Rosner B, Speizer FE, Hennekens CH. Postmenopausal estrogen therapy and cardiovascular disease. Ten-year follow-up from the nurses' health study. N Engl J Med. 1991;325:756-762.

143. Grady D, Rubin SM, Petim DB, Fox CS, Black D, Etringer B, Ernster VL, Cummings SR. Hormone therapy to prewent disease and prolong life in postmenopausal women. Anm Intern Med. $1992 ; 117: 1016-1037$.

144. Hulley S, Grady D, Bush T, Furberg C, Herringron D, Riggs B, Vittinghoff E. Randomized trial of esrrogen plus progestin for secondary prevention of coronary hearr disease in posrmenopausal womer. Heart and Estrogen/progestin Replacement Study (HERS) Research Group. Jama. 1998:280:605-613. 
145. Herrington DM, Reboussin DM, Brosnihan KB, Sharp PC, Shumaker SA, Snyder TE, Furberg CD, Kowalchuk G. Sruckey TD, Rogers W], Givens DH, Waters D. Efects of estrogenteplacement on the progression of coronary-artery atherosderosis. $N$ Engl $/$ Med. 2000;343:522-529.

146. Waters DD, Alderman EL, Hsia J, Howard BV, Cobb FR, Rogers WJ, Ouyang P. Thompson P. Tardif JC, Higginson L, Bitner V, Steffes M, Gordon DJ, Proschan M, Younes N, Verter JI. Effects of Hormone Replacement Therapy and Antioxidant Vitamin Supplements on Coronaty Atherosclerosis in Postmenopausal Women: A Randomized Controlled Trial. JAMA. $2002 ; 288: 2432-2440$.

147. Beral V. Breasr cancer and hormone-replacement rherapy in the Million Women Srudy. Lantet. $2003 ; 362: 419-427$.

148. Bush TL, Barrett-Connor E, Cowan LD, Criqui MH, Wallace RB, Suchindran CM, Tyroler HA, Rifkind BM. Cardiovascular mortality and noncontraceptive use of estrogen in women: results from the Lipid Research Clinics Program Follow-up Srudy. Circularion. 1987,75:1102-1109.

149. Mendelsohn ME, Karas RH. Estrogen and the blood vessel wall. Cur Opwin Cardiol. $1994: 9.619-626$.

150. Effects of estrogen or estrogen/progestin regimens on hearr disease risk factors in postmenopausal women. The Postmenopausal Estrogen/Progestin Interventions (PEPI) Trial. The Writing Group for the PEPI Trial. Jama. 1995;273:199-208.

151. Nabulsi AA, Folsom AR, White A, Parsch W, Heiss G, Wu KK, Szklo M. Associarion of hormone-replacement therapy with various cardiovascular risk factors in postmenopausal women. The Atherosclerosis Risk in Communities Study Investigators. N Engl/ / Med 1993;328:1069-1075.

152. Effects on haemostasis of homone replacement therapy with transdermal estradiol and oral sequential medroxyprogesterone acetate: a 1 -year, double-blind, placebo-controlled study. The Writing Group for the Estradiol Cloung Factors Sudy. Thromb Haemost. 1996;75:476-480).

153. Salomaa V, Rasi V, Pekkanen J, Vahrera E, Jauhiainen M, Vartiainen E, Ehnholm $C_{\text {o }}$ Tuomilehto J, Myllyla G. Association of homone replacement therapy with hemostatic and other cardiovascular

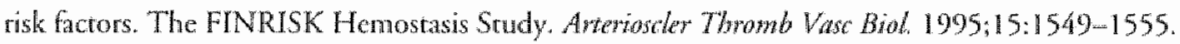

154. Sugioka K, Shimosegawa $Y$, Nakano M. Estrogens as matural antioxidants of membrane phospholipid peroxidation. FEBS Lett. 1987;210:37-39.

155. Liehr JG. Antioxidant and prooxidant properties of estrogens. J Lab Clim Med. $1996 ; 128: 344345$.

156. Shwaery GT, Vita JA, Keaney JF, Jr. Antioxidant protection of LDL by physiological concentrations of 17 beta-estradiol. Requirement for estradiol modification. Circulation. 1997:95:1378-1385.

157. Santanam N, Shern-Brewer R, McClatchey R, Castellano PZ, Murphy AA, Voelkel S, Parthasarathy S. Estradiol as an antioxidant: incompatible with its physiological concentrations and function. J Lipid Res. 1998;39:2111-2118.

158. Sack MN, Rader DJ, Cannon RO, 3rd. Oestrogen and inhibition of oxidation of low-density lipoproteins in postmenopausal women. Lamet. 1994;343:269-270.

159. Amal JF, Clamens S, Pechet C, Negre-Salvayre A, Allera C. Girolami JP, Salvayre R, Bayard F. Ethinylestradiol does not enhance the expression of nitric oxide synthase in bovine endorhelial cells but increases the release of bioactive nirric oxide by inhibiting superoxide anion production. Proc NaW Acad Sci US A. 1996;93:4108-4113. 
160. Proudler A. Ahmed AI, Crook D, Fogelman I, Rymer IM, Stevenson JC. Hormone replacement therapy and serum angiotensin-converting-enzyme activity in postmenopausal women. Lancet. $1995 ; 346: 89-90$.

161. Ylikorkala O, Orpana A, Puolakka J. Pyonala T, Vinikka L. Postmenopausal homonal replace ment decreases plasma levels of endorhelin-1. J Clin Endocinol Metab. 1995;80:3384-3387.

162. Nickenig $G$, Baumer AT, Grohe $C$, Kahiert $S$, Srrehlow K, Rosenkranz S, Stablein A, Beckers F, Smits JF, Daemen MJ Wetter H, Bohm M. Estrogen modulates AT1 receptor gene expression in vitro and in vivo. Circulation. 1998;97:2197-2201.

163. Best PJ, Berger PB, Miller VM, Lerman A. The effect of estrogen replacement therapy on plasma nitric oxide and endothelin-1 levels in postmenopausal women. Ann Intem Med. 1998;128:285-288.

164. Farhat MY, Lavigne MC, Ramwell PW. The vascular protective effects of estrogen. Faseb J. $1996 ; 10: 615-624$.

165. Moncada S, Higgs A. The L-arginine-nitric oxide parhway. N Engl/ Med 1993;329:2002-2012.

166. Curtiss LK. ApoE in atherosclerosis : a protein with multiple hats [editorial, comment]. Arteriorder Throwb Viss Biol 2000;20:1852-1853.

167. Caulin-Glaser T, Garcia-Cardena $G$, Sarrel P. Sessa WC, Bender IR. 17 beta-estradiol regulation of human endothelial cell basal nitric oxide release, independent of cyrosolic $\mathrm{Ca} 2+$ mobilization. Circ Res. 1997;81:885-892.

168. Chen Z, Yuhanna IS, Galcheva-Gargova Z, Kaas RH, Mendelsohn ME, Shaul PW. Estrogen receptor alpha mediates the nongenomic acrivation of endothelial nitric oxide synthase by estrogen Ipublished erratum appears in J Clin Invest 1999 May; 103(9):1363]. I Clin Invest. 1999;103: $401-406$.

169. Williams JK, Adams MR, Herrington DM, Clarkson TB. Short-term administration of estrogen and vascular responses of atherosclerotic coronary arteries. J Am Coll Cardiol 1992;20:452-457.

170. Sudhir K, Ko E Zellner C, Wong HE, Hutchison SI, Chou TM, Chatterjee K. Physiological concentrations of estradiol attenuate endothelin 1 -induced coronary vasoconstriction in vivo. Circulation. 1997:96:3626-3632.

171. Reis SE, Gloth ST, Blumenthal RS, Resar JR, Zacur HA, Gerstenblith G, Brinker JA. Erhinyl estradiol acutely atrenuates abnormal coronary vasomotor responses to acerylcholine in postmenopausal women. Cinculation. 1994;89:52-60.

172. Gilligan DM, Quyyumi AA, Cannon RO, $3 \mathrm{rd}$. Effects of physiological levels of estrogen on coronary vasomotor funcrion in postmenopausal women. Citculation. 1994;89:2545-2551.

173. Gilligan DM, Badar DM, Panza JA, Quyyumi AA, Cannon RO, 3rd. Acute vascular effects of estrogen in postmenopausal women. Circulation. 1994;90:786-791.

174. Lieberman EH, Gerhard MD, Uehata A, Walsh BW, Selwyn AP, Ganz P, Yeung AC, Creager MA. Estrogen improves endothelium-dependen, How-mediated vasodilation in postmenopausal women. Anw Intern Med. 1994; 121:936-941.

175. Collins P, Rosano GM, Sarrel PM, Ulrich L, Adamopoulos S, Beale CM, McNeill JG, Poole-Wilson PA. 17 beta-Estradiol attenuates acetylcholine-indaced coronary arterial constriction in women bur not men with cotonary heart disease. Circtilation. 1995;92:24-30. 
176. Guetra V, Quyyumi AA, Prasad A, Panza JA, Wadawiw M, Cannon RO, 3rd. The role of nimic oxide in coronary wascular effects of estrogen in postmenopausal women. Circulation. 1997:96: 2795-2801.

177. Blumenthal RS, Heldman AW, Brinker JA. Resar JR, Coombs VI, Gloth ST, Gerstenblith G, Reis $S E$. Acute effects of conjugated estrogens on coronary blood flow response to acerycholine in men. Am J Cardial 1997;80:1021-1024.

178. Reis SE, Bhoopalam V, Zell KA, Counihan PJ, Smith A], Pham S, Murali S. Conjugated estrogens acutely abolish abnormal cold-induced coronary vasoconstriction in male cardiac allografts. CinctuLation. 1998;97:23-25.

179. Weiner CP, Lizasoain I, Baylis SA, Knowles RG, Chartes IG, Moncada S. Inducrion of calciurn-dependent nitric oxide synthases by sex hormones. Proc Nat Acad Sci USA. 1994:91:5212-5216.

180. Binko J, Majewski. H. 17 beta-Estradiol reduces vasoconstriction in endothelium-denuded rat aortas through inducible NOS. Am J Physiol. 1998:274:H853-859.

181. Williams JK, Adams MR, Klopfenstein HS. Estrogen modulates responses of atherosderotic coronary arteries. Circulation 1990;81:1680-1687.

182. Williams JK, Honore EK, Adams MR. Contrasting effects of conjugated estrogens and tamoxifen on dilator responses of atherosclerotic epicardial coronary arteries in nonhuman primates. Cinculatron 1997;96:1970-1975.

183. Herrington DM, Braden GA, Williams JK, Morgan 'TM. Endothelial-dependent coronary vasomotor responsiveness in postmenopausal women with and without estrogen replacemem therapy. Am J Cardiol. 1994:73:951-952.

184. Roque M, Heras M, Roig E, Masotri M, Rigol M, Betriu A, Balasch J, Sanz G. Short-term effects of cransdermal estrogen replacement therapy on coronary vascular reactivity in postmenopausal women with angina pectoris and normal results on coronary angiograms. I Am Coll Cardiol. $1998: 31: 139-143$.

185. Krasinski K, Spyridopoulos I, Asahara T, van der Zee R, Isner JM, Losordo DW. Estradiol accelerates functional endothelial recowery after arterial injury. Circulation. 1997:95:1768-1772.

186. Cormwell TL, Anold E, Boerth NJ, Lincoln TM. Inhibicion of smooth muscle cell growti by nitric oxide and acriwation of CAMP-dependenc protein kinase by CGMP. Am J PHisiol 1994;267: C1405-1413.

187. Kolodgie FD, Jacob A, Wilson PS, Carlson GC, Fatb A, Verma A, Virmani R. Estradiol artenuates dinected migration of vascullar smooth muscle cells in vitro. Am / Pathol 1996; $148: 969-976$.

188. Bhalla RC, Toth KF, Bhaty RA. Thompson L.P. Sharma RV. Estrogen reduces proliferation and agonist-induced calcium increase in coronary artery smooth muscle cells. Am / Pbysiol 1997;272: H. $996-2003$.

189. Iafrat MD, Karas RH, Aronovitz M, Kim S, Sullivar TR, Jr. Lubahn DB, O. Donnell TF J, Korach KS, Mendelsohn ME. Estrogen inhibits the vascular injury response in estrogen receptor alpha-deficient mice. Nat Med. 1997:3:545-548.

190. Keaney JF, Jr., Shwaery GT, Xu A, Nicolosi RJ, Loscalzo J, Foxall TL., Vita JA. 17 beta-estradiol preserves endothelial vasodilaror funcrion and limits low-densiry lipoprotein oxidation in hypercholesterolemic swine. Circulation. 1994;89:2251-2259. 
191. Sullivan TR, Jr, Karas RH, Aronovic M. Faller GT, Ziar IP, smith JJ, O Donnell TF J, Mendelsohn ME. Estrogen inhibirs the response-to-injury in a mouse carotid artery model. J Clm Invert. 1995;96:2482-2488.

192. Bourasaa PA, Milog PM, Gaynor B], Breslow JL, Aiello RJ. Estrogen reduces atherosclerocic lesion development in apolipoprovein E-deficient mice. Prac Nat Acad SeI USA. 1996:93:10022-10027.

193. Clatkson TB, Anthony MS, Kein KP. Effects of estrogen treatment on arterial wall structure and function. Drowgs 1994,47:42-51.

194. Levine RL, Chen S], Durand J, Chen YF, Oparil S. Medroxyprogesterone atrenuates estrogen-mediated inhibition of neointima formation after balloon injury of the rat carotid artery. Circulation. $1996 ; 94: 2221-2227$.

195. Oparil S, Levine RL, Chen S], Durand J, Chen YF. Sexually dimorphic response of the balloon-injused rat carotid artery to hormone treatment. Cinculation. 1997:95:1301-1307.

196. Nathan L, Chatdhuri G. Estrogens and atherosclerosis. Anwu Rev Phamacol Toxicol. $1997 ; 37: 477-515$

197. [Pare G. Krust A, Karas RH, Dupon S, Aronovitz M, Chambon P, Mendelsohn ME. Estrogen Receptor-alpha Mediates the Protective Effects of Estrogen Against Vascular Injury. Circ Res. 2002;90:1087-1092.

198. Hodgin JB, Krege JH, Reddick RL, Korach KS, Smithies O, Maeda N. Estrogen receptor alpha is a major mediator of 17 beta-estradiol's atheroprotective effects on lesion size in Apoe-f-mice. $f$ Clin Invest. 2001;107:333-340.

199. Stuehr DJ. Mammalian nitric oxide synthases. Biochim Bioplys Acta. 1999;1411:217-230.

200. Schmidr K, Werner ER, Mayer B, Wachter H. Kukoverz WR. Terrahydrobiopterin-dependent formation of endothelium-derived relaxing factor (nitric oxide) in aorric endorhelial cells. Biochem f. 1992;281:297-300.

201. Wever RM, van Dam T, wan Rijn HJ, de Groot F, Rabelink TJ. Terahydrobiopterin regulates superoxide and nitric oxide generation by recombinant endothelial nitric oxide synthase. Biochem Bioplys Res Common. 1997:237:340-344

202. Vasquez. Vivar J, Kalyanaraman B, Martasek P. Hogg N, Masters BS, Karoui H, Tordo P. Pritchatd $K A_{3}$, Jr. Superoxide generation by endothelal nitric oxide synthase: the influence of cofactors. Proc Nat Acud Sci US A. 1998995,9220-9225.

203. Shinozaki K, Kashiwagi A. Nishio Y., Okamura T, Yoshida Y, Masada M, Toda N, Kik.kawa R. Abnotmal biopterin merabolism is a major cause of impaired endothelium-dependent relaxation through nitric oxide/O2- imbathance in insulin-resistant rar aorta. Diabetes. 1999;48:2437-2445.

204. Kerr S, Brosnan MJ, Melntyre M, Reid JL, Dominiczak AF, Hamilton CA. Superoxide anion production is increased in a model of generic hypertension: role of the endothelium. Hypentention. 1999:33:1353-1358.

205. Cosentino F, Pakton S, d'Uscio LV, Wener ER, Werner-Felmayer G, Moreau P, Malinski T", Luscher TF. Tetrahydrobiopterin alters superoxide and nitric oxide release in prehypertensive rats. J Clin linest 1998;101:1530-1537.

206. Srroes E, Kastelein J, Cosentino F, Erkelens W, Wever R, Koomans H, Luscher T, Rabelink T. "etrahydrobiopterin restores endothelial function in hypercholesterolemia. J Chin Invest. 1997;99: 41- 46 . 
207. Heiter T, Brockhoff C, Mayer B, Warnholz A, Mollnau H, Henne S, Meinera T, Mundel T. Tetrahydrobiopterin improves endohelum-dependent wasodilation in chronic smokers : evidence for a dysfuncrional nitric oxide synthase. Cir Res. 2000;86:B36-41.

208. Kinoshita $\mathrm{H}_{3}$ Milstien 5 , Wambi $\mathrm{C}$. Katusic ZS. Inhibition of retahydrobiopterin biosynthesis impairs endothelium-dependen relaxations in canine basilar artery. Am / Physol 1997273: $1718-724$.

209. Wever RM, Luscher TF, Cosentino F, Rabelink T]. Atherosderosis and the two faces of endothelial nitric oxide synthase. Circulation. 1998;97:108-112.

210. Stroes ES, van Faassen EE, Yo M, Marasek P, Boer P, Govers R, Rabelink T). Folic acid reverts dysfunction of endothelial nitric oxide synthase. Cir Rer. 2000;86:1129-1134.

211. Verhaar MC, Wever RM, Kastelein JJ, van Dam T, Koomans HA. Rabelink Ty. 5-methyltetrahydrofolate, the active form of folic acid, restores endothelial function in familiat hypercholesterolemia. Cimculation. 1998;97:237-241.

212. Loscalzo J. Folate and nitrate-induced endothelial dysfunctioni a simple treatment for a complex pathobiology. Cinculation. 2001;104:1086-1088.

213. Heller R, Unbehaun A, Schellenberg B, Mayer B, Werner-Felmayer G, Werner ER. L-ascorbic acid potentiates endothelial nitric oxide synthesis via a chemical stabilization of tetrahydrobiopterin. J Biol Chem. 2001;276:40-47.

214. Kaufman $S$. Some metabolic relationships berween biopterin and folate: implicarions for the "methyl trap hyporhesis". Newrochem Res. 1991;16:1031-1036.

215. Yin ZL, Dusting G. A nitric oxide donor (spermine-NONOate) prevents the formation of neoincima in rabbit carotid artery. Clin Exp Phantatol Pbysiol. 1997;24:436-438.

216. Kaul S, Cercek B, Rengstrom J, Xu XP. Molloy MD. Dimayuga P, Parikh AK. Fishbein MC, Nilsson J, Rajavashisth TB, Shah PK. Polymeric-based perivascular delivery of a nitric oxide donor inhibits intimal thickening after balloon denudation arterial injury: role of nucleat factor-kappaB. I Ary Coll Cardiol. 2000,35:493-501.

217. Guo JP, Panday MM, Consigny PM, Lefer AM. Mechanisms of vascular preserwation by a novel NO donor following rat carorid artery intimal injury. Am / Physol. 1995:269:H1122-1131.

218. Wolf YG, Rasmussen LM, Sherman X, Bundens WP. Hye R. Nirroglycerin decreases medial smooth messece cell proliferation after arterial balloon injury. / Vast Swrg 1995:21:499-504.

219. Seki ], Nishio M, Kato Y, Motoyama Y, Yoshida K. FK409, a new nitric-oxide donor:, suppresses smoorh muscle proliferation in the rat model of balloon angioplasty. Awheroscterosix. 1995:117: $97-106$.

220. Maffia l', Lanaro A, Sorrencino R, Lippolis L, Maidlo FM, del Soldaro P, lalenti A, Cirino G. Ben.eficial effects of $\mathrm{NO}$-releasing derivawe of furbiprofen (HCT-1026) in rat model of vascular in. jury and restenosis. Aremoscley Thromb Vac Biol 2002;22:263-267.

221. Yu ], Rudic RD, Siessa WC. Nitric oxide-releasing aspirin decreases vascular injury by teducing inflammation and promoting apoptosis. Lab Invest. 2002;82:825-832.

222. Kalinowski $M$. Affke H, Bergen S, Klose KJ, Barry J, Wagner HJ. Compatative trial of local pharmacorherapy with L-arginine, $\mathrm{r}$-hirudin, and molsidomine to reduce restenosis afrer balloon angioplasty of stenotic rabbir iliac arteries. Radiology. $2001 ; 219: 716-723$. 
223. Napoli C, Cirno G, Del Soldaro P, Sorrentino R, Sica V, Condorelli M, Pinro A, Ignarro LI. Ef fects of nimic axide-rcleasing aspirin wersus aspirin on restenosis in hypercholesterolemic mice. Proc Nat Acad Sai U S A. 2001;98:2860-2864.

224. Rolland PH, Bartoli IM, I'iquer P, Mekkaoni C, Nor SH, Moulin G, Amabile P, Mesana T. Local delivery of NO-donor molsidomine post-PTA improves haemodynamics, wall mechanics and histomotphometry in atherosderotic porcine SFA. Eury J Vasc Endouasc Surg. 2002:23:226-233.

225. Kullo IJ Simari RD, Schwartz. RS. Vascular gene transfer : from bench to bedside Arteriouter Thromb Vasc Biol. $1999 ; 19: 196-207$.

226. Channon KM, Blazing MA, Sherty GA, Potts KE, George SE. Adenowiral gene transfer of nirtic oxide synthase: high level expression in human vascular cells. Cardiovar Res. 1996;32:962-972.

227. Ooboshi H, Chu Y. Rios CD, Faraci FM, Davidson BL, Heistad DD. Altered vascular function after adenovirus-mediated overexpression of endothelial nitric oxide synchase. Am J Pbysiol. 1997;273:H265-270.

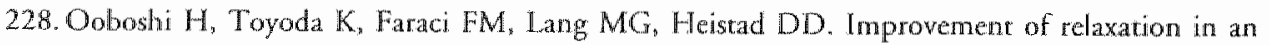
attheroscleroric antery by gene transfer of endothelial nitric oxide syothase. Arterioscler Thromb Vasc Biol 1998; 18:1752-1758.

229. Mozes G, Kullo IJ, Mohacsi TG, Cable DG, Specror DJ, Crotty TB, Gloviczki P, Karusic ZS, T OB. Ex viwo gene transfer of endothelial nirric oxide synthase to atherosderoric rabbit aortic rings improves relaxations to acerylcholine. Atheroxclerosis. 1998;141:265-271.

230. Cable DG, O'Brien T. Kullo IJ, Schwart RS, SchaffHV, Pompili VT. Expression and function of at recombinant endorhelial nicric oxide synthase gene in porcine coronary arteries. Cardiowate Res. $1997,35: 553-559$.

231. Chen AF, O'Brien T", Tsutsui M, Kinoshita H, Pompili VJ, Crotry TB, Spector DJ, Katusic ZS. Expression and function of recombinant endothelial nitric oxide synthase gene in canine basilar arvery. Cin Res. 1997:80:327-335.

232. Cable DG, Caccitolio JA, Pearson PJ, O'Brien T, Mullany CJ, Daly RC, Orszulak TA, Schaff HV. New approaches to prevention and trearment of radial artery graft vasospasm. Circulation. 1998;98:I J 15-21: discussion II21-12.

233. Cable DG, Pompili VJ, O'Brien T, Schaff HV. Recombinant gene transfer of endothelial nitric oxide synthase angments coronary artery relaxanions during hypoxia. Ciwadstion. 1999:100: 11335-339.

234. Tsutsui M. Chen AF, O'Brien T, Crotry TB, Karusic ZS. Adventitial expression of recombinant eNOS gene restores NO production in areries withour endothelium. Arteriowler Thromb Vasc Brol. $1998 ; 18: 1231-1241$.

235. Janssens $S$. Flaherty $D$, Nong $Z$, Varenne $O$, van Pelt $\mathbb{N}$, Haustermans $C$, Zoldhelyi P, Gerard R, Collen D. Human endothetial nitric oxide synthase gene transfer inhibirs vascular smooth muscle cell proliferation and neointima formation after balloon injury in rats. Circulation. 1998;97: $1274-1281$.

236. won der Leyen HE, Gibbons GH, Morishira R, Lewis NP, Zhang L, Nakajima M, Kaneda $\mathrm{Y}$, Cooke IP. Dzau VI. Gene therapy inhibiring neointimal wascular lesion: in vivo transfer of endothelial cell nirric oxide symhase genc. Proc Nat Acad Si US A. 1995:92:1137-1141. 
237. Shears LL, 2nd, Kibbe MR, Murdock AD, Billiar TR, Lizonova A, Kovesdi I, Wattins SC, Treng E. Efficient inhibition of intimal hyperplasia by adenovirus-mediated inducible nirric oxide synthase gene rransfer to rats and pigs in wivo. / Am Coll Sug 1998;187:295-306.

238. Varenne $\mathrm{O}$, Pislaru $S$, Gillijns $H$, Van Pelt $N$, Gerard $R D$, Zoldhelyi $P$, Van de Werf F, Collen D. Janssens SP. Local adenowirus-mediated transfer of human endothetial nitric oxide synthase rèduces luminal narrowing after coronary angioplasty in pigs. Cortudation. 1998;98:919-926.

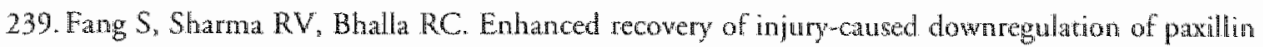
protein by eNOS gene expression in rar carotid artery. Mechanism of NO inhibition of intimal hyperplasia? Anteviosder Thromb Vasc Biol. 1999;19:147-152.

240. Channon KM, Qian H. Neplioueva V, Blazing MA, Olmez E, Sherry GA, Youngblood SA. Pawloski J, McMahon T, Stander JS, George SE. In viwo gene transfer of nitric oxide synthase enhances vasomotor funcrion in carotid arteries from nomal and cholesterol-Fed rabbits. Cinchation. 1998:98:1905-1911.

241. Chen AF, Jiang SW, Croty TB, Tsursui M, Smin LA, O'Brien T, Katusic ZS. Effects of in wivo adventitial expression of recombinant endothelial nitric oxide syuthase gene in cerebral arteries. Proc Nat Acad Sci US A. 1997;94:12568-12573.

242. Kullo IJ, Mozes G, Sclawartz RS, Glovicala P, Tsutsui M, Katusic ZS, T OB. Enhanced endorhelium-dependent relaxations after gene transfer of recombinan endothelial nitric oxide synthase to rabbit carotid arteries. Hypertension. 1997;30:314-320.

243. Kullo IJ, Mozes G, Schwantz RS, Gloviczki P, Crorry TB, Barber DA, Karusic ZS, OBrien T. Adventitial gene transfer of recombinant endothelial nitric oxide synthase to rabbit carorid arteries alters vascular reacrivicy. Cirulation. 1997;96:2254-2261.

244. Qian H, Neplioueva V, Shetry GA, Channon KM, George SE. Nitric oxide synthase gene therapy rapidly reduces adhesion molecule expression and inflammatory cell infiltation in carotid arteries of cholesterol-fed rabbits. Cinculation. 1999;99:2979-2982.

245. Shears LL, Kawaharada N, Tzeng E, Billiar TR, Watkins SC, Kovesdi I, Lizonova A, Pham SM. Inducible nitric oxide synthase suppresses the development of allograft arteriosclerosis. $/ \mathrm{Clin} / \mathrm{In}$ vest. 1997;100:2035-2042.

246. Marsumoto T, Komori K, Yonemirsu Y, Morishita R, Sueishi K, Kaneda Y, Suginachi K. Henagglutinating virus of Japan-liposome-mediated gene ransfer of endothelial cell nitric oxide synthase inhibirs intimal hyperplasia of canine wein grafts under conditions of poor runoff, / Vase Surg. 1998;27:135-144.

247. Janssens SP. Bloch KD. Nong Z, Gerard RD, Zoldhelyi P, Collen D. Adenoviral-mediated transfer of the human endothelial nitric oxide synthase gene reduces acute hypoxic pulmonary wasoconstriction in rats. 7 Clin Inoest. 1996;98:317-324.

248. Gaballa MA, Goldman S. Oxerexpression of endothelium nitric oxide synthase reverses the dimin. ished wasorelaxation in the hindlimb vasculature in ischernic heart failure in vivo. $\int \mathrm{Mol} C \mathrm{Cel}$ Cardiol. 1999;31:1243-1252.

249. Lake-Bruse KD, Faraci FM, Shesely EG, Maeda N, sigmund CD, Heistad DD. Gene transfer of endonelial nirric oxide synthase (eNOS) in wOS-deficient mice. Am / Prysiol 1999;277:H770-776.

250. Alexander MY, Brosnan MI. Hamilton CA, Downie P, Devlin AM, Dowell F, Marrin W. Prentice HM, OBrien T, Dominiczak AF. Gene ransfer of endothelial nitric oxide synthase im- 
proves nitric oxide-dependent endothelial function in a hypertensive rar model. Covdiowat Rex. $1999+437798-807$

251. Nakane H. Miller F), Jt., Faraci FM, Toyoda $K$, Heistad DD. Gene transfer of endothelial nitric oxide synthase reduces angiotensin II-induced endothel hal dysfunction. Hypertension. 2000;35: 595-601.

252. Land DD, Faraci FM, Miler FJ. Jr., Heisrad DD. Gene transfer of endothelial nitric oxide synthase improves relaxation of carovid arteres from diaberic rabbits. Circulation. 2000;101:1027-1033.

253. Myers PR, Tanner MA. Vascular endotheliah cell regulation of extracellular matrix collagen: role of nitric oxide Arterioscley Thromb Vase Biol 1998; 18:717-722.

254. Tarry WC, Makhoul RG. L-arginine improves endorhelitum-dependent vasorelaxation and reduces intinal hyperplasia after balloon angioplasty. Anterioscler Thromb. 1994:14:938-943.

255. Guo K, Andres V, Walsh K. Nitric oxide-induced downregulation of Cdk2 activity and cyclin A gene transcription in wascular smooth muscle cells. Choulation. 1998;97:2066-2072.

256. Sharma RV, Tan E, Fang S, Gurjar MV, Bhalla RC. NOS gene transfer inhibits expression of cell cycle regulatory molecules in vascular smooth muscle cells. Am J Physol 1999;276:H1450-1459.

257. Gurjar MV, Sharma RV, Bhalla RC. eNos gene transfer inhibits smooch muscle cell migration and Mmpr2 and Mmp-9 acrivity. Arterioscler Thromb Vasc Brol. 1999;19:2871-2877.

258. Pollman MI, Yamada $T$, Horiuchi M, Gibbons GH. Vasoactive substances regulate vascular smooth musde cell apoptosis. Countervailing influences of nitric oxide and angiotensin II. Cin Res. 1996:79:748-756.

259. Meng YY, Trachtenburg ], Ryan US, Abendschein DR. Potentiation of endogenous nitric oxide with superoxide dismurase inhibits platelet-mediated thrombosis in injured and stenotic arteries. $J$ An Coll Cardiol. 1995;25:269-275.

260. Fingerle J, Au YP. Clowes AW, Reidy MA. Intimal lesion formation in rar carotid arteries after endothelial denudation in absence of medial injury. Arterioscleroses. 1990;10:1082-1087.

261. Saniabadi AR, Umemura K, Marsumoto N, Sakuma S, Nakashima M. Vessel wall injury and artetial thrombosis induced by a photochemical reaction. Thromb Haemost. 1995;73:868-872.

262. Kikuchi S, Umemura K. Kondo K. Saniabadi AR, Nakzshima M. Photochemically induced endothelial injury in the mowe as a screening model for inbibitors of wascular intimal thickening. Arterioscher Thromb Vas. Biol. 1998;18:1069-1078.

263. Betz E, Schlore W. Responses of vessel watls to chronically applied electrical stimuli. Batic Res Cardiol. 1979774:10m20.

264. Banai S, Shou M, Correa R, Jaklitsch MT, Douek PC, Bonner RF, Epsein SE, Unger EF. Rabbit ar model of injury-induced arrerial smooth muscle cell proliferation. Kinetics, reproducibiliny, and implicarions. Cotc Res. 1991:69:748-756.

265. Richardson M, Haton MW, Buchanan MR, Moore S. Wound healing in the media of the normolipenic rabbic carotid artery injured by air drying or by balloon carbeter dewendothelialization. Am / Pathol. 1990;137:1453-1465.

266.5mon DI, Dhen Z, Seifert P, Fdeman ER, Ballantyne CM, Rogers C. Decreased neointimal formation in Mac-1 (- $/$ ) mice reveals a role for inflammation in vascular repair afrer angioplasty. $)$ Clin Inwest 2000;105:293-300. 
267. Bood RF, Martin JF, Honey AC. Hassall DG, Beesley JE, Moncada S. Rapid dewelopment of acherosclerotic lesions in the rabbit carotid artery induced by perivascular manipulation, Adseroscierosis. $1989 ; 76: 257-268$.

268. De Meyer GR, Bult H, Van Hoydonck AE, Jordaens FH, Buyssens $N$, Herman AG. Neointimat formation impars endothelial muscarnic receptors while enhancing prostacyclin-mediatred responses in the rabbit carorid arrery. Cir Res. 1991;68:1669-1680.

269. Kockx MM, De Meyer GR, Jacob WA, Bull H. Herman AG. Triphasic sequence of neointimal formation in the cuffed carotid artery of the rabbic. Arteriosder Thromb. 1992;12:1447-1457.

270. Soma MR, Donerti E, Parolini $C$, Mazzini $G$, Ferrari $C$ Fumagalli R, Paoletti R. HMG CoA reducrase inhibitors. In vivo effects on carotid intimal dhickening in nomocholesterolemic rabbirs. Anterioscler Thromb. 1993;13:571-578.

271. Lindner V, Fingerle J, Reidy MA. Mouse model of arterial injury. Cort Res. 1993:73:792-796.

272. Channon KM, Qian H, George SE. Nitric oxide synthase in arherosclerosis and vascular injury: insighrs from experimental gene therapy. Arterioscler Thromb Vurc Biol. 2000;20:1873-1881.

273. Laufs U. Beyond lipid-lowering: effects of statins on endothellal nitric oxide. Eur/ Clin Plywacol. 2003;58:719-731.

274. West NE, Qian H, Guzik TJ, Black E, Cai S, George SE, Channon KM. Nirric oxide synthase (nNOS) gene transfer modifies venous bypass graft remodeling: effecrs on vascular smooth muscle cell differentiarion and superoxide producrion. Circulation. 2001:104:1526-1532. 

Reduced endothelium-dependent relaxations and impaired acute flow-induced vasodilation do not compromise chronic flow-related arterial remodeling in hyperlipidemic mice

Henny M. Schulten, Rob H.P. Hilgers, Paul M.H. Schiffers, Agnieszka Strzelecka,

Gregorio E. Fazzi, Ebo D. de Muinck, and Jo G.R. De Mey. 


\begin{abstract}
Background - We sought to determine whether mild and severe hyperipidemia which are associated with endothelial dysfunccion are accompanied by an impairment of both acure flow-induced diation and flow-related arterial remodeling.
\end{abstract}

Methods and Results - Ex-wiwo agonist- and flow-induced vasomotor responses were determined in carotid arteries of 6 months old apolipoprotein $\mathrm{E}$ deficient mice $\left(a p o E^{-j}\right.$ on a Western diet and in wild type (WT) mice on a Western dier in comparison to the responses in WT animals. Structural parameters were measured in carotid arteries of shammperated animals and in carond arteries of animals four weeks after unilarenal carotid artery ligation. Plasma cholesterol levels were more than 10-and 5-fold increased in respecrively apoE $\mathrm{E}^{-1-}$ and WT both maintained on Western rype diet as compared to WT" on normal chow. Acerylcholine-induced relaxarions were reduced in carovid artery segments of apo $\mathrm{E}^{-1-}$ on diet and WT on diet. Indomethacin normalized the acerylcholine-induced relaxations. Flow-induced dilation of isolated carorid arteries was reduced in apo $\mathrm{E}^{-1}$ on dier as compared to borh WT and WT on dier at all flow rates $(P<0.01)$. In apo $E^{-/ 2}$ on diet, indomethacin increased flow-induced dilation at high flow rates. In WT on diet, indomethacin reduced flow-induced dilation at low flow rates $(P<0.05)$, but nor at high flow rates. Media cross-sectional area of the carrorid artery was significantly smaller in apo $\mathrm{E}^{-/-}$on diet and WT on diet than in WT on normal chow. Unilateral carotid artery ligarion stopped blood flow in the ipsilateral vessel and resulted in a significant increase of blood flow in the contralateral vessel. This increase in blood flow was significantly smaller in apo $\mathrm{E}^{-1-}$ on diet and WT on diet. In the chronically hyperperfused contralateral arteries outer diameter was significantly increased in all experimental groups. In the occluded artery, outer diameter was markedly and comparably reduced after 4 weeks in all experimental groups. This inward remodeling was accompanied by marked media hypertrophy in. apolet/ on dier and WT on dier while no media hypertrophy was seen in WT on normall chow.

Condwsions - In hyperlipidemic mice chronic flow-related arterial remodeling is preserved despite reduced endothelium-dependent relaxarions and blunted acute flow-induced dilation. 


\section{INTRODUCTION}

Arteries respond to chronic alterations in blood flow by structural luminal diameter adjustments in order to normalize wall shear stress. " 2 In general, chronic blood flow reductions lead to a narrowing of lumen diameter, while chronically elevated blood flow results in a widening of arteries. ${ }^{3-6}$ Flow-relared remodeling occurs in skeleral muscle arteries during endurance training 7 and in uterine arteries during pregancy. ${ }^{8}$ Also, in pathological situations, flow-related remodeling is involved, for instance, in coronary collateral. arteries after ischemia of the heart. ${ }^{9}$ Outward remodeling has been proposed to represent a natural defense mechanism that prevents the impact of a growing neointima or atherosclerotic plaque on blood flow. ${ }^{10,11}$ Arterial remodeling in response to imposed, developmental or physiological changes in blood How was demonstrated to be endothelium-dependent. ${ }^{5,12}$

On an acute basis, the endothelium responds to increases in blood flow by releasing vasoactive compounds, such as nitric oxide (NO), ${ }^{13}$ prostacyclin, ${ }^{14}$ bradykinin ${ }^{15}$ and endothelium-derived hyperpolarizing factor (EDHF). ${ }^{16}$ It has been proposed that sustained flow-induced dilation ultimately gives rise to structural arterial wall adjustments. In a broad variety of conditions, such as hypercholesterolemia, acute endothelium-dependent vasomotor responses to agonists and blood flow were found to be reduced as a result of decreased bioavailability or functional antagonism of endothelium-derived nirric oxide. ${ }^{17-20}$ Whether an altered endothelium-dependent reactivity predisposes to abnormalities in flow-related remodeling is unclear.

We hyporhesized that during hypercholesterolemia flow-related remodeling is decreased because of endothelial dysfunction. To address this issue, endorhelial (dys)function was evaluated in young adult ( 6 months) wild type (WT) mice on a normal diet, wild type mice on high fat "Western-type" dier and apolipoprotein E-deficient (apoE ${ }^{-6}$ ) mice on diet. Arterial remodeling in response to altered blood flow was studied after unilateral carotid artery ligation. Functional and structural analyses were concentrated on the central part of the common carotid arteries which was devoid of neointima and atherosclerotic plaques in all experimental groups. In this study we document (1) reduced endothelium-dependent relaxations in apoEon diet and WT animals on diet (2) impaired acure flow-induced dilation in severely hyperlipidemic apoE $\mathrm{E}^{-/}$mice on diet and altered acure flow-induced dilation in mildly hyperlipidemic WT on diet and (3) comparable structural arterial diameter responses to chronically altered blood flow in the different strain and diet groups. 


\section{MATERIALS AND METHODS}

\section{ANIMALS}

Procedures used in these experiments were approved by the University of Maastricht Animal Care and Ethical Commitree. We studied C56BL/6I mice (WT) and homozygous apoE-deficient mice (with a $129 \times$ C57BL/6J background and backcrossed 9 generations on a C57BL/6J background; Charles River Laboratories, the Nerherlands). Mice had free access to tap water and were maintained from weaning on either regular mouse chow (Hope Farms, Woerden, the Netherlands) or on a high fat "Western-type" of diet (15\% cacao butter, $2 \%$ cholate, $0.25 \%$ chollesterol, $40.5 \%$ sucrose, $10 \%$ corn starch, $1 \%$ corn oil and $5.95 \%$ cellulose; Hope Farms, Woerden, the Netherlands).

\section{LIPID PROFILE}

Plasma cholesterol and triglyceride levels were determined in duplicate by using a colorimetric assay (CHOD-PAP 1442341 and GPO-PAP 701912, respectively, La Roche) in arterial plasma samples obtained at 6 months of age.

\section{UNILATERAL CAROTID ARTERY LIGATION}

Male wild type mice on normal chow (WT, $n=24$ ), male wild type on a high fat diet (WT on diet, $\mathrm{n}=24$ ), and male apo $\mathrm{E}^{-/}$on a high fat diet (apoE ${ }^{-/}$on diet, $\mathrm{n}=24$ ) were anestherized with ketamine/xylazine (100 and $10 \mathrm{mg} / \mathrm{kg} \mathrm{s.c.).} \mathrm{At} \mathrm{an} \mathrm{age} \mathrm{of} 5$ months, both common carotid arteries were exposed through a midline incision in the neck. In half of each experimental group, the Jeft carotid artery was ligated with 5.0 surgical suture just caudal to the carotid bifurcation as described (Figure 1). 2, 3 In the other animals a sham operation was performed in which mice were operated as described above omitting the left carotid artery ligation. Only two animals ( 1 WT on diet and $1 \mathrm{apoE}^{-1-}$ on diet) died after the ligation procedure.

\section{BLOOD FLOW MEASUREMENTS}

For weeks after sham surgery or unilateral carotid artery ligation animals were anesthetized again with ketamine/xylazine and body temperature was maintained at $37.5^{\circ} \mathrm{C}$ by a thermostatically controlled heating platform. Both the left and right carotid artery blood flow were recorded. We used a transit-rime ultrasonic How-probe $(0.5 \mathrm{~mm} V$-series, Transonic Systems, Ithaca, NY, USA) that was mounted on a micro-manipulator positioned halfway between the aortic arch and the carotid artery bifurcarion. The pulsatile blood flow signal was recorded and 


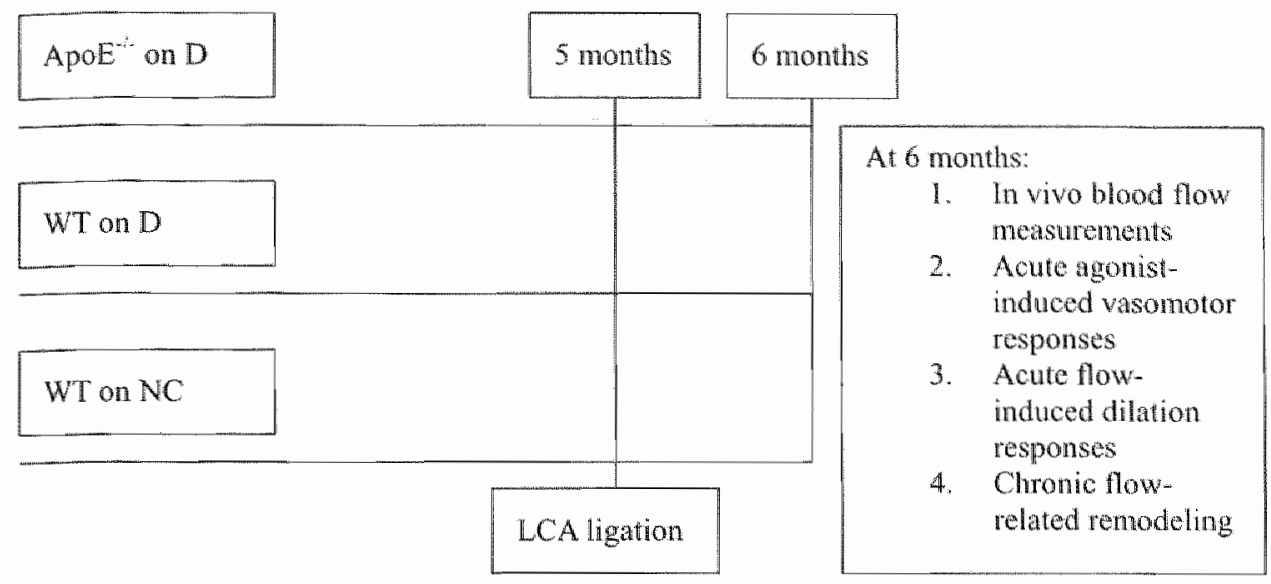

Figure 1. Scheme of experimental setup. Unilateral leficarotid antery (LCA) ligation was performed at an age of 5 months in apoE-deficient mice maintained on a Western type diet (apo $\mathrm{E}^{-1 /}$ on D), wild type on D (WT on D) and WT on normal chow (WT on NC). For weeks after carotid artery ligation mice in vivo blood flow meaurements were performed and mice were sacrificed with subsequent $a x$-pino measurements as indicared in scheme.

mean blood flow was obrained by averaging during a 5-minute period for both the left and right carotid artery of each animal.

\section{ACUTE AGONIST-INDUCED VASOMOTOR RESPONSES}

Two-millimeter segments of the freshly isolated carotid arteries were mounted (steel wires, diameter $40 \mu \mathrm{m}$ ) in a myograph organ bath (Danish Myotechnology Aarhus, Denmark) for isometric tension measurements. Organ barhs were filled with a Krebs-Ringer bicarbonate buffer (composition in mmol/L: $\mathrm{NaCl}, 118.5 ; \mathrm{KCl}, 4.7$; $\mathrm{MgSO}_{4} .7 \mathrm{H}_{2} \mathrm{O}, 1.2 ; \mathrm{KH}_{2} \mathrm{PO}_{4}, 1.2 ; \mathrm{NaHCO}_{3}, 25.0 ; \mathrm{CaCl}_{2}, 2.5 ;$ glucose, 5.5), maintained at $37^{\circ} \mathrm{C}$, and aerated with $95 \% \mathrm{O}_{2}$ and $5 \% \mathrm{CO}_{2}$. The arterial segments were stretched until maximal contractile responses to $63 \mathrm{mmol} / \mathrm{L}$ potassium solution were achieved (optimal diameter). After 30 minutes of equilibration, concentration-response curves for phenylephrine (Phe) $\left(10^{-8}\right.$ to $10^{-4.5} \mathrm{~mol} / \mathrm{L}$, half $\log$ units) were constructed and relaxing effects of ACh $\left(10^{-8}\right.$ to $10^{-5} \mathrm{~mol} / \mathrm{L}$, half $\log$ units $)$ and Na-nitroprusside (SNP) $\left(10^{-10}\right.$ to $10^{-4.5} \mathrm{~mol} / \mathrm{L}$, half $\log$ units) were evaluated. Parallel experiments were performed in the absence and presence of $100 \mu \mathrm{mol} / \mathrm{L}$ $N^{G}$-nitro-arginine-methyl ester (L-NAME) or $10 \mu \mathrm{mol} / \mathrm{L}$ indomethacin. All drugs were obrained from Sigma Chemical Co (St. Louis, MO, USA). 
Carotid arteries of sham operated animals were isolated and placed in a thermostated bath containing Hanks balanced salt solution (HBSS, composition in mmol/L: $\mathrm{NaCl}, 1.43 ; \mathrm{KCl}, 4.7 ; \mathrm{MgSO}_{4} .7 \mathrm{H}_{2} \mathrm{O}, 1.2$; glucose, 5.5; HEPES, 15) aerated with $95 \% \mathrm{O}_{2}$ and $5 \% \mathrm{CO}_{2}$. They were cannulated at both ends and then perfused continuously in vitro in a system in which flow and pressure could be modified independently. ${ }^{21}$ At the beginning of each experiment, arteries were equilibrated for 45 minutes at $70 \mathrm{mmHg}$ and perfused with an intraluminal flow rate of $10 \mu \mathrm{L} / \mathrm{min}$. The carotid arteries were maintained under a transmural pressure of $70 \mathrm{mmHg}$ during the experiments. The outer diameter was determined with a binocular lens connected to a video-camera system (Stemmer, VS 450). The pressure at both ends of the segment was monitored using pressure transducers. Intraluminal pressure upstream and downstream of the artery was controlled by means of a pressure servo-control (Living System Instruments, Inc), so that flow could be increased without changing intraluminal pressure. The presence of the endothelium was evaluated by assessing the dilation in response to acetylcholine (ACh; $1 \mu \mathrm{mol} / \mathrm{L}$ ) during constriction induced by phenylephrine (Phe; $30 \mu \mathrm{mol} / \mathrm{L}$ ).

All experiments evaluating the response to increases in intraluminal flow rate were performed in the presence of Phe $(30 \mu \mathrm{mol} / \mathrm{L})$ with or without indomethacin $(10$ $\mu \mathrm{mol} / \mathrm{L}$; a cyclooxygenase inhibitor that does not discriminate between COX-1 and COX-2) or indomethacin and L-NAME $(100 \mu \mathrm{mol} / \mathrm{L})$. Each inhibitor combination was incubated for at least 30 minutes in the intraluminal and extraluminal perfusions. When the constriction to Phe was stable for at least 10 minutes, the intraluminal flow rate was increased in a stepwise manner from 10 to $800 \mu \mathrm{L} / \mathrm{min}$. The flow was augmented to the next level until a stable diameter was reached.

\section{PRESSURE DIAMETER CURVES}

The relationship between transmural pressure and diameter was determined in carotid arteries exposed to normal blood flow (sham-operated mice) and after 4 weeks exposure to elevated or drastically reduced blood flow (unilateral carotid artery ligation). Isolated arteries were incubated in vitro for 30 minutes in $\mathrm{Ca}^{2+}$-free HBSS containing SNP $(10 \mu \mathrm{mol} / \mathrm{L})$, which abolished the smooth muscle tone. Passive diameter curves were obtained by increasing intraluminal pressures in 10 mmHg steps from 20 to $150 \mathrm{mmHg}$. Outer diameter was recorded at 2 minutes after each pressure increment. After reaching the highest pressure, pressure was returned to $100 \mathrm{mmHg}$ and the artery was incubated in phosphate-buffered $(\mathrm{pH}$ 7.4) formaldehyde (4\%) for overnight fixarion. All drugs were obtained from Sigma Chemical Co. (St. Louis, MO, USA).

Outer diameter was expressed as a function of distending pressure. To determine the maximal outer diameter, pressure-diameter curves were analyzed by a four-parametric 
logistic sigmoidal curve fitting $\left(Y=Y_{\min }+\left(Y_{\max }-Y_{\min }\right) /\left(1+10^{(\log \quad \text { E50-X).Hillslope }))}\right)\right.$ (Graphpad Prism 2.01, San Diego, CA, USA). The correlation coefficient of each individual curve fit exceeded 0.99 in all cases.

\section{MORPHOMETRY}

To concentrate histological analyses on the central part of the carotid arteries, fixed carotid arteries were cur in half and both parts were embedded side by side in paraffin. Cross-secrions $(4-\mu \mathrm{m})$ were stained with Lawson's solution (Boom BV, Meppel, the Netherlands), which highlights the elastic laminae. Media cross-sectional area (CSA) was determined, using video images generated by a Zeiss axioscope (Zeiss, Germany), a standard CCD camera (Sony) and commercial software (JAVA 1.21, 『andell Scientific, Corte Madera, CA, USA).

\section{DATA ANALYSIS}

Results are shown as means \pm SEM. Statistical significance of differences berween mouse strains and diets was evaluated using analysis of variance (ANOVA) followed by a post-hoc comparison with student Newman-Keuls significant difference test. For arteriograph studies $n$ refers to the total number of vessels out of 5-7 mice. Comparisons between two groups were made with the Student's t-test. All statistics were performed using SigmaStat software. A P-value of $<0.05$ was considered significant.

\section{RESULTS}

\section{GENERAL OBSERVATIONS}

Body weight was significantly larger for apo $\mathrm{E}^{-/}$on diet and WT on diet compared to wild type mice on normal chow (NC) (Table 1). Cholesterol levels were significantly higher in apoEt- on diet and WT on diet than W'T on NC. Plasma triglycerid levels were significantly elevared in the apoE ${ }^{-\%}$ on diet as compared to the other two groups (Table 1). Carotid artery blood flow did not differ in sham-operated animals berween the left and right carotid arteries and among the three experimental groups (Table 1). This excludes the possibility that group differences in arterial reacrivity to agonists and flow rates were due to different in vivo blood flow velociries berween groups. Distal ligation of the left carotid artery stopped blood flow in the vessel and resulted in a substantial increase in blood flow through the contralateral artery in all experimental groups. This increase in blood flow was less pronounced in apo $\mathrm{E}^{-/}$on diet and WT on diet than in WT on NC (Table 1). 
Table 1. Characteristics of mice

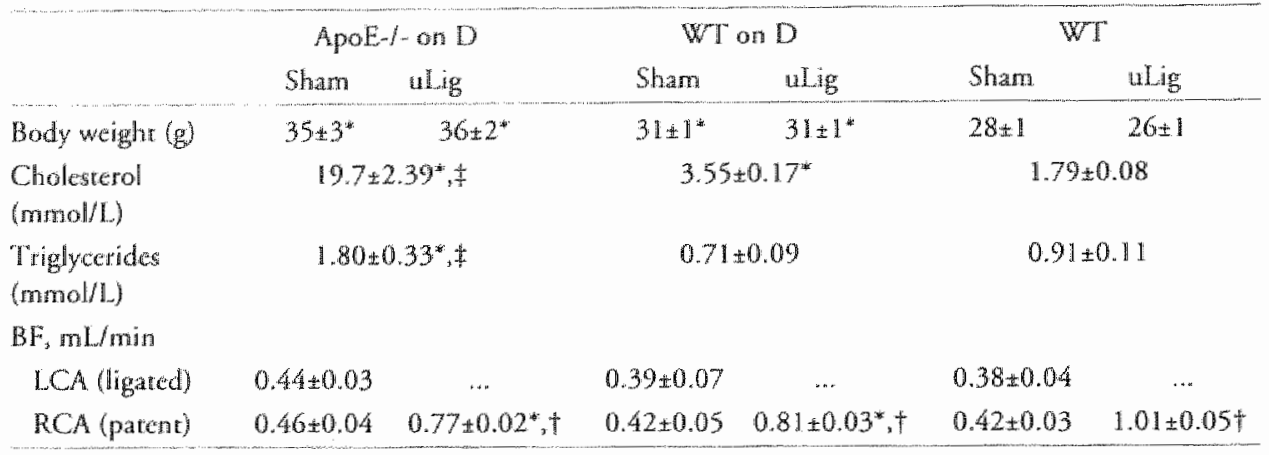

Values are means: $S E M(n-7-12)$ and were monitored at 4 weeks after surgery. Points ... indicate BF recording below derection limit. " $\mathbb{P}^{3}<0.05$ ws WT; $\dagger$ P<0.05 ws sham 4 P<0.05 vs WT on Wertern type diet (D). uLig; unilateral carotid artery ligation; BF, blood flow; LCA, left carotid artery; RCA, right canotid antery.

Despite clear atherosclerosis of the aortic arch in $\mathrm{apoE}^{-1-}$ on diet (not shown), central parts of all common carotid arteries of both apoE $\mathrm{E}^{-\mathrm{z}}$ on diet and WT on dier were free from plaques and neointima.

\section{ACUTE CONTRACTILE REACTIVITY}

Sensitivity to the contractile effect of the $\alpha_{1}$-adrenergic agonist phenylephrine was comparable in carorid artery segments of apoE ${ }^{-\%}$ on diet, WT on diet and WT (data not shown).

Acetylcholine reduced phenylephrine-induced contraction in all experimental groups (Figure 2). Micromolar concentrations of acetylcholine resulted in contractile responses in apoE $\mathrm{E}^{-/}$on diet and WT on diet, indicating endorhelial dysfunction (Figure 2). The sensitivity and maximal relaxing effect of Na-nitroprusside did, however, not differ berween experimental groups, showing unaltered response to nitric oxide in the diet groups (data not shown).

The effect of L-NAME on maximal phenylephrine-induced contraction was less pronounced in W'T on diet (from $2.5 \pm 0.4$ to $2.7 \pm 0.2 \mathrm{~N} / \mathrm{m}$ ) than in apoE $E^{-t-}$ on diet (from $1.2 \pm 0.2$ to $2.4 \pm 0.2 \mathrm{~N} / \mathrm{m}$ ) and WT (from $1.9 \pm 0.2$ to $3.1 \pm 0.4 \mathrm{~N} / \mathrm{m}$ ), suggesting reduced bioavailability of endogenous $N O$ in WT animals on diet as compared to the other groups (Figure 3). In apo $\mathrm{E}^{-\%}$ on diet indomethacin enhanced the contractile response to phenylephrine (from $1.20 \pm 0.20$ to $1.96 \pm 0.2 \mathrm{~N} / \mathrm{m}$, $\mathrm{P}<0.05$ ), but did not change the sensitivity (Figure 3). However, in WT on diet indomethacin significantly lowered the maximal contractile response (maximal contraction $2.49 \pm 0.4$ to $0.90 \pm 0.1 \mathrm{~N} / \mathrm{m})$ and sensitivity $\left(-\log \mathrm{IC}_{50} \times 6.7 \pm 0.1\right.$ to $5.8 \pm 0.3, \mathrm{P}<0.01$ ) to phenylephrine (data not shown).

In the presence of indomethacin acetylcholine-induced relaxarions were improved in the diet groups (Figure 3, maximum values from $37.4 \pm 15.8 \%$ to $82.6 \pm 3.7 \%(P<0.05)$ and from $44.0 \pm 6.6 \%$ to $91.6 \pm 2.0 \%(\mathrm{P}<0.05)$ in apoE $-1-$ on 

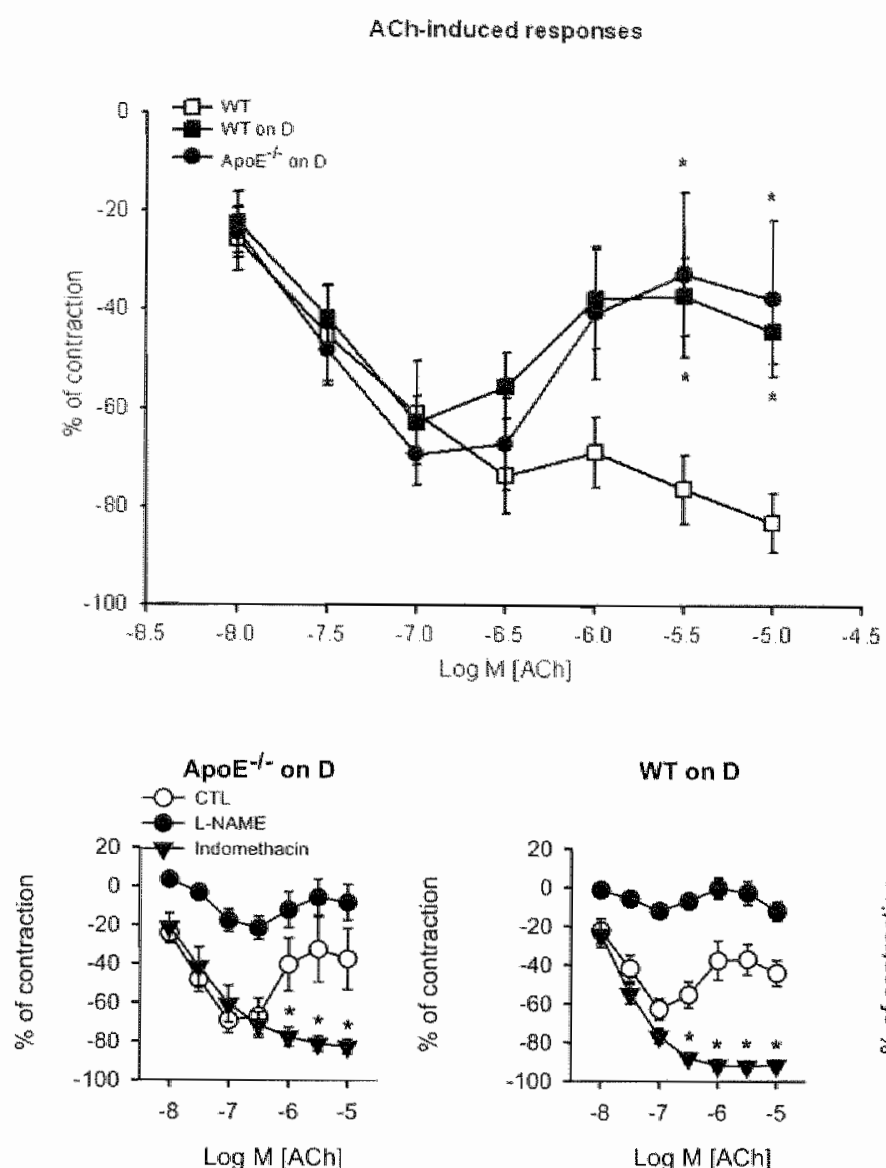

WT on D

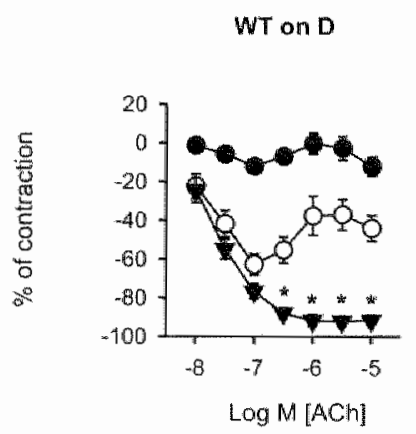

Figure 2. Indothelial dystuncthon. Relaxing effects (expressed as percentage inhibition of wessels contracted widh 30 wnoll. Phe) of acemblholine (ACh) in carotid segments of apo: $\mathrm{E}^{-t}$ on Dor WT on a high fat Western rype dier (WT on D) and of WT nulce maintained on normal chow $(\mathrm{W} T) * P<0.05$ vs WT.

Figure 3. Relaxing effects (expressed as percentage inhibirion of yessels contrated with 30 umol/L Phe)

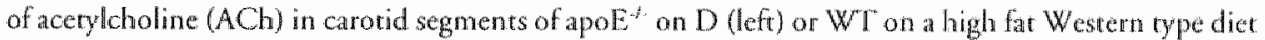
(WT on D. middle) and of WT mice maintained on normal chow (WT, right). Experiments wetre performed in the absence (open circles) and presence of 100 mollL. L-NAME (closed circles) or $10 \mu \mathrm{mol} / \mathrm{L}$. indomerhacin (triangles). Means $\mathrm{SEM}(\mathrm{n}=5-9)$. " $\mathrm{P}<0.05$ ws CT1.

diet and WT on diet respectively). Indomethacin had no effect on acerylcholine-induced relaxations in WT on normal diet (Figure 3).

\section{ACUTE FLOW-INDUCED ARTERIAL DILATION}

The outer diameter of the carotid arteries maintained at a transmural pressure of 70 $\mathrm{mmHg}$ was significantly larger in apo $\mathrm{E}^{-1-}$ on $\operatorname{dier}(564 \pm 7 \mu \mathrm{m}, \mathrm{P}<0.001)$ than in WT on diet $(511 \pm 4 \mu \mathrm{m})$ and WT $(518 \pm 6 \mu \mathrm{m})$.

In carotid arteries of apoE $\mathrm{E}^{-1-}$ on diet, constriction in response to $30 \mu \mathrm{M}$ phenylephrine was significantly smaller than that observed in the other two groups. In WT 


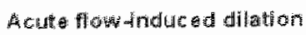

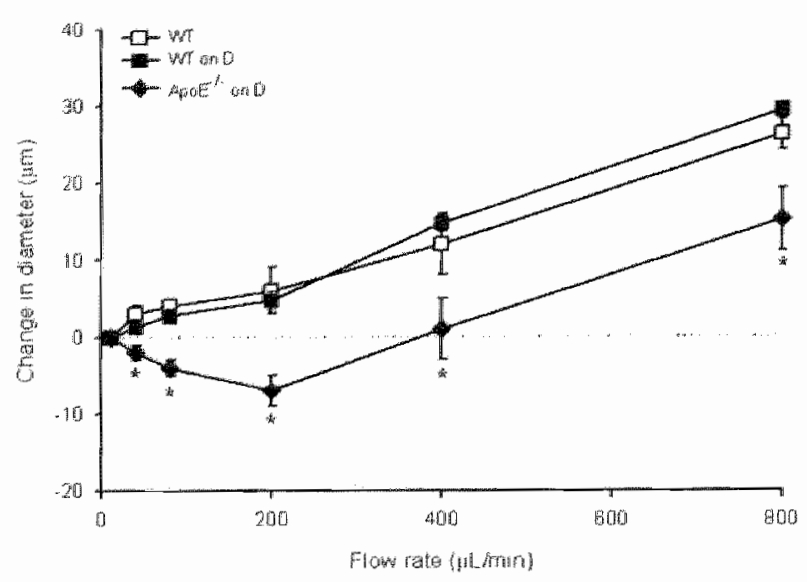

Figure 4. Acute flow-induced changes in diameter (pm) in perfused carotid arterics of WT (open squares), WT on D (closed squares) and apok $\mathrm{E}^{-2-}$ on $\mathrm{D}$ (criangles). * P<0.01 ws WT and WT on D. Means 5 SEM $(n=6-10$ vessels from $5-7$ mice). ${ }^{*} \mathrm{P}<0.05 \mathrm{vs}$ WT and WT on D.

on diet and WT, exposure to phenylephrine caused comparable constriction (data not shown). Dilations in response to $\mathbb{N a}$-nitroprusside $(10 \mu \mathrm{M})$ were indistinguishable between groups (data not shown).

The increase in diameter in response to increasing intraluminal flows was significantly reduced in apoE-t- on diet and even a flow-dependent constriction was observed at low flow rates (Figure $4 ; 40-200 \mu \mathrm{L} / \mathrm{min}, \mathrm{P}<0.05$ vs $10 \mu \mathrm{L} / \mathrm{min}$ ), while no differences in flow-diameter responses were seen between WT on diet and WT.

In apoE-t- on diet, indomethacin augmented flow-induced dilation at a flow rate of $800 \mu \mathrm{L} / \mathrm{min}$, suggesting a role for vasoconstrictor cyclooxygenase products (Figure 5). In W/T on diet, indomethacin reduced dilation in response to low flow rates (between 10 and $400 \mu \mathrm{L} / \mathrm{min}, \mathrm{P}<0.01$ ws control), suggesting the involvement of flow-induced vasodilator cyclooxygenase products (Figure 5). In WT, this effect was less pronounced ( $a t 40$ and $80 \mu \mathrm{L} / \mathrm{min} ; \mathrm{P}<0.05$ ws control). The combination of indomethacin and L-NAME did not alter flow-induced dilation as compared to incubations with indomethacin alone in all experimental groups.

\section{CHRONIC FLOW-RELATED CAROTID ARTERY REMODELING}

Pressure-diamerer curves of left and right carotid arteries of sham-operated mice were identical and did not differ between experimental groups. Therefore, the average values of the left and right arteries were pooled in Figure 6. Irrespecrive of strain and diet, manipulation of blood flow did not lead to neointima formation in the central portion of the carotid arteries. Media cross-sectional area (CSA) was significantly smaller in sham-operated apoE $E^{-/-}$on dier and WT on dier $\left(16.7 \pm 0.7 \times 10^{3} \mu^{2}\right.$ and $\left.18.1 \pm 0.5 \times 10^{3} \mu \mathrm{m}^{2}\right)$ compared to WT $\left(24.5 \pm 1.4 \times 10^{3} \mu \mathrm{m}^{2}\right.$, Figure 7$)$. In mice of all experimental groups unilateral carotid artery ligation resulted in (1) a reduc- 
Hot E

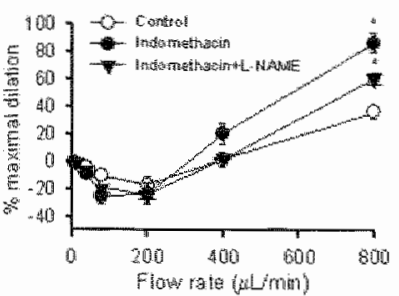

WT और 0

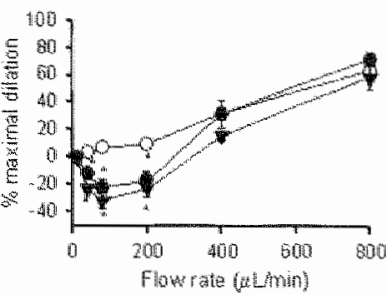

w

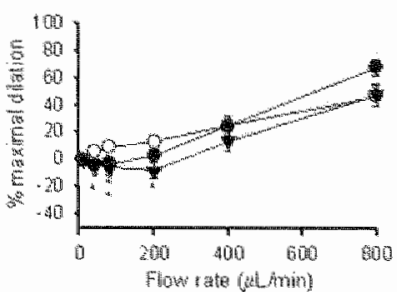

Figure 5. Acure flow-induced tesponses (expressed as percentage of maximal dilation to $10 \mu \mathrm{M} S \mathrm{SN}$ ) in caronid arteries of apo $E^{*}$ on D (left), WT on D (middle) and WT (right). Experiments were performed in the absence (open circles) and presence of $10 \mu \mathrm{mol} / \mathrm{L}$ indomethacin (closed circles) or indomethacin and 100 umol/L L-NAME (triangles). * $P<0.05$ ws control. Means $S E M \quad(n=5-10$ from 5-7 mice).

ApoE.' on D

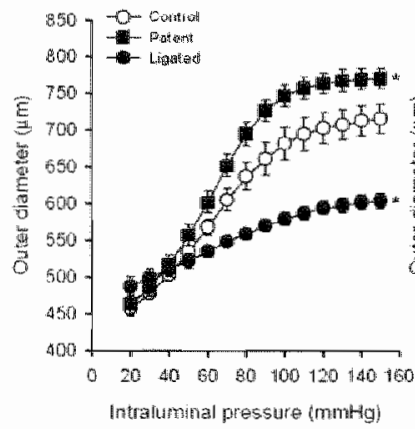

WT an D

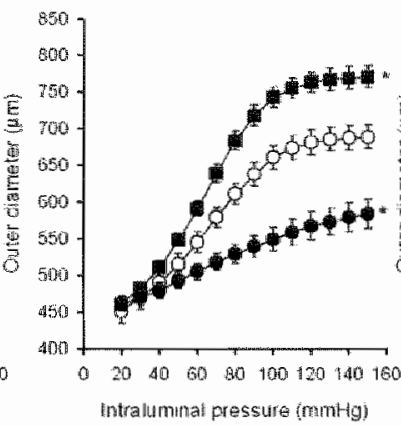

wil

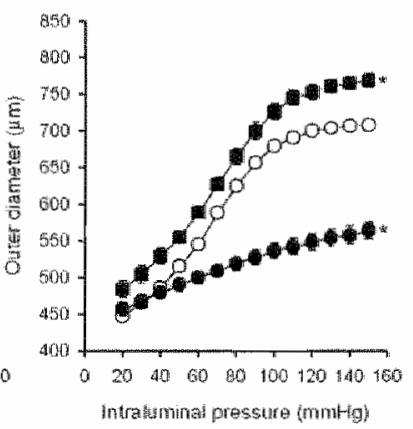

Figure 6. Effects of unilateral carotid artery ligation on pressure-diameter relationships in ligated arteries (closed circles) and in contralateral (patent) arteries (closed squares) for apoE $\mathrm{E}^{*}$ on D (left), WT on D (middle) and WT (right). ${ }^{*}<<0.05$ vs concrol. Findings are shown as means 4 SM $(n=6-12)$ for ligated (closed symbols) and sham-operated animals (open symbols).

A poE

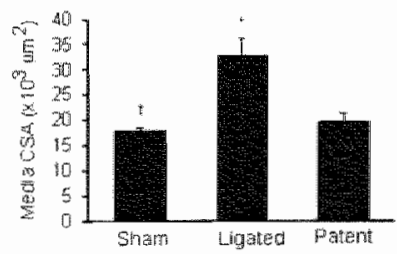

WT an D

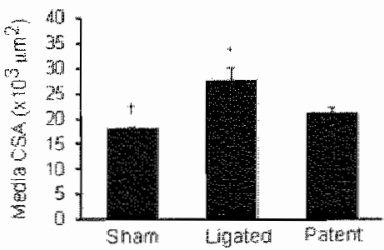

wT

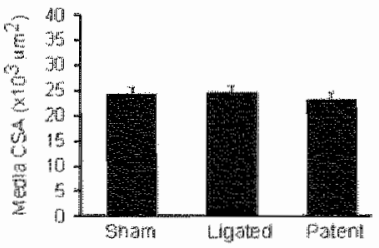

Figure 7. Effects of unilarenal carotid artery ligation on media cross-sectional area (CSA) in apot on $\mathrm{D}$ (lefry, WT on D (middle) and for WT mice maintained on normal chow (right). Findings are shown as means \pm SEM ( $\mathrm{n}=6-12)$. Sham, sham-operated carorid areries, Ligared, experimentally ligated left carotid arwery; Patent, contralateral right carotid artery. ${ }^{*} \mathrm{P}<0.001$ vs sham, $+\mathrm{P}<0.001$ ws WT. 
tion of diamerer and an increase in stiffness of the ligated arteries and (2) in diameter increases of the hyperperfused arteries after 4 weeks (Figure 6). In all experimental groups chronic hyperperfusion did not result in a significant change of media CSA (Figure 7). In the ligated arteries of both WT on dier and apo $E^{-/-}$on diet a significant media hypertrophy was seen while in WT no change in media CSA was observed compared to sham-operated arteries (Figure 7 ).

\section{DISCUSSION}

This study demonstrates that both impaired endothelial function and impaired acute flow-induced vasodilation do not compromise flow-related arterial remodeling in hyperlipidemic mice.

In apoE ${ }^{-1-}$ mice, hypercholesterolemia, arherosclerosis and endothelial dysfunction are markedly increased by a "Western-type" high fat diet. ${ }^{22}$ Plasma cholesterol levels are markedly elevated because of increased levels of VLDL and LDL, atherosclerosis develops at sites comparable to those in human atherosclerosis, plasma nitrite levels, a marker for $\mathbb{N O}$ activity in vivo, are reduced, and impaired arterial endothelium-dependent vasodilator responses to agonists have been observed. 17,18 Here, we addressed whether this encails a reduced capacity to respond ro acute and chronic changes in flow.

\section{ENDOTHELIAL DYSFUNCTION}

The endorhelial dysfunction observed in 6 months adult WT and apoE ${ }^{-*}$ both on diet was restricted to increased contractile responses to high concentrations of acerylcholine.

The mechanisms underlying endothelial dysfunction in hyperlipidemia and atherosclerosis in general, and in apo ${ }^{-/}$in particular, remain controversial. Expres sion and protein levels of endothelial nitric oxide synthase and the production of $\mathrm{NO}$ in the atherosclerotic rabbit aorta appear to be increased rather than decreased.23,24 Inactivation of NO by superoxide anions produced in excess as a result of the incorpotation of lipids within the endothelium, a deficiency in co-factors for NO-synthase, such as rerrahydrobiopterin, 25 and increased levels of asymmetric dimethylarginine (ADMA) ${ }^{20}$ could be possible alternatives.

However, increased inactivation of endothelium-derived $\mathrm{NO}$ is not supported by the normal relaxing responsiveness to $\mathrm{Na-nitroprusside} \mathrm{and} \mathrm{the} \mathrm{identical} \mathrm{sensiriza-}$ rion to this NO-donor substance by L-NAME (not shown), which we observed in apot-t- compared to WT. Reported effects of an endothelin receptor antagonist on endothelial dysfunction ${ }^{4}$ rather suggest a role for an endothelium-derived contractile factor, such as endothelin-1. 
In this study we show that wasoconstrictor cyclooxygenase products are involved in the increased contractile response to acetylcholine in mice on a Western type diet. Nevertheless, NO may be indirectly involved in this increased contractile response to acerylcholine, because the contractile component in the acetylcholine response was not observed in the presence of L-NAME in carotid artery segments of WT on diet and apo $E^{* /-}$ on diet. Under pathological circumstances such as hypercholesterolemia in which vascular oxidase systems are upregulated, superoxide anions are produced which reacr with $\mathrm{NO}$ to form peroxynitrite (ONOO). Gupte et a ${ }^{26}$ found that superoxide in the presence of an $\mathrm{NO}$ donor elicited tromboxane ( $\mathrm{T} \times \mathrm{A}_{2}$ )-mediared. constriction in the rat coronary circulation. A likely mechanism by which ONOOmay interfere with arachidonic acid metabolism is inactivation of $\mathrm{PGI}_{2}$ synthase, ${ }^{27}$ thus promoting synthesis of TxA $\mathrm{A}_{2}$, as suggested by Bagi et al. ${ }^{28}$

\section{ACUTE FLOW-INDUCED DILATION}

Flow-induced dilation has been shown to be endothelium-dependent. ${ }^{15,29}$ In line with other studies we found that vasodilator prostaglandins (PGs) and other factors not characterized, possibly EDHF and bradykinin, are involved in flow-induced dilation. ${ }^{15,16,30-33}$

In the severely hypercholesterolemic apoE $\mathrm{E}^{-1}$ on dier, flow-induced dilation was impaired at all flow rates tested. At low flow rates, flow-induced constricting factors distinct from $\mathrm{PGs}$ and $\mathrm{NO}$ are involved. At the highest flow rate, however, vasoconstrictor cyclooxygenase products were involved. A candidate constrictor at low flow rates is endothelin-1. ${ }^{34}$ Lüscher et al ${ }^{17}$ reported that endothelin-l production is increased in apoE-deficient mice. Furthermore, an early study reported evidence for endothelial flow-dependent release of free radicals unrelated to cyclooxygenase or nitric oxide synthase activities in aortae of normocholesterolemic rabbits. ${ }^{35}$ Under normal and mild hypercholesterolemic conditions these flow-dependent free radicals can be buffered by efficient scavenging systems, but possibly not in excessive free radical production in severe hyperlipidemia. In mildly hypercholesterolemic WT on diet, flow-induced dilation seemed to be maintained by increased production of vasodilator PGs at low llow rates.

\section{CHRONIC FLOW-RELATED REMODELING}

We used unilateral carotid artery ligation ${ }^{3,4}$ to measure arterial flow-related remodeling. In this model, a growing neointima develops close to the ligation. ${ }^{3}$ This involves P-selectin, ${ }^{36} \mathrm{PDGF}^{37}$ and $\mathrm{bFGF}^{36-38}$ and is regionaliy restricted. In WT $\mathrm{C} 57 \mathrm{BL} / 6]$ mice, it does nor extend into the center of the common carotid arkery. ${ }^{39}$ As judged from our findings, neointima formation in response to the absence of flow is not markedly increased by a high-fat diet or by severe hyperlipidemia resulting from apoE-deficiency. 
We focussed on the central part of the vessel because it represents the structural response of the intact wall to altered shear stress without the complicaring influences of a neointima or atherosclerotic plaque. ${ }^{40}$

As noted previously, ${ }^{4}$ total carotid artery blood flow was largely maintained in wild type mice after unilateral carotid artery ligation due to a compensatory increase in contralateral blood flow. Pre-existing collaterals, that interconnect the facial, cranial and cerebral areas, perfused by both carotid arteries form the anatomical basis for this shunting. ${ }^{41}$ In arteries of immature juvenile animals, chronic increases in blood flow lead to compensatory outward hypertrophic remodeling, i.e., an increase in both lumen diamerer and wall mass whereby wall shear stress and circumferential wall stress are normalized. 12,42 However, the ability of the vessel wall to respond to altered blood flow diminishes with age. 12,42 We recently reported that doubling of blood flow did not alter carotid artery media mass or diameter after 4 weeks in mature 4-month old 129/Sv mice.4 Despite mild to severe hyperlipidemia, altered flow-induced dilation and reduced contralateral shunting of blood flow, the flow-loaded carotid arteries of these mice displayed a significant increase in diameter and no media hypertrophy. This structural response tends to normalize wall shear stress, thereby preserving the integrity of the endothelium. These findings in hyperlipidemic mice are in line with our previous observations in vimentin-deficient mice. ${ }^{4}$ In these mice, absence of the intermediate cytoskeleton filament results in impaired endothelium-dependent flow-induced dilation, ${ }^{43}$ but does not entail a blunting of flow-related arterial remodeling. ${ }^{4}$

\section{IN SUMMARY}

In mild and severely hyperlipidemic mice, endothelium-dependent relaxations were reduced and acute flow-induced vasomotor function was altered and markedly impaired, respectively. Neither, however, impeded arterial structural responses to unilateral carotid artery ligation.

\section{REFERENCES}

1. Kamiya A, Togawa T. Adaptive regulation of wall sheat stress to flow change in the canine carotid attery. Ams J Plywiol. 1980;239:H14;-21.

2. Zarims CK, Zatina MA, Giddens DP, Ku DN, Glagov S. Shear stress regulation of artery lumen diameter in experimental atherogenesis. $/$ Vac Surg. 1987:5:413-420.

3. Kumar $A$, Lindner $V$. Renodeling with neointima formation in the monse carotid antery after cessation of blood flow. Arveriosder Thromb Vassc Biol. 1997; 17:2238-2244.

4. Schiffers PM, Henrion D, Boulanger CM, Colucci-Guyon E, Langa-Vuves F, van Essen H, Fazzi GE, Levy BI, De Mey JG. Altered flow-induced arterial remodeling in wimentin-deficient mice. Arteriosder Thromb Vasc Biol. 2000:20:61 1-616. 
5. Langille BL, O'Donnell F. Reductions in arterial diameter produced by chronic decreases in blood flow are endothelium-dependenc. Science. 1986;231:405-407.

6. Ben Driss A, Benessiano I, Poitevin P. Levy BI, Michel JB. Arrerial expansive remodeling induced by high How rares. Am J Physiol 1997:272:H851-858.

7. Segal SS, Kurjiaka DT, Caston AL. Endurance craining increases arterial wall thickness in rats. $J$ Appl Pbysal. 1993;74:722-726.

8. Osol G, Cipolla M. Pregnancy-induced changes in the three-dimensional mechanical propertics of pressurized rat uteroplacental (radial) arteries. An J Obstet Gynecol. 1993;168:268-274.

9. Schaper W, Iro WD. Molecular mechanisms of coronaty collateral wessel growth. Circ Res 1996:79:911-919.

10. Glagov $S$, Weisenberg E, Zarins CK, Stankunavicius R, Koletris GJ. Compensatory enlargement of human atherosderotic coronaty arteries. $N$ EnglJ Med 1987;316:1371-1375.

11. Pasterkamp G, Schoneveld AH, van Wolferen W, Hillea B, Clarijs RJ, Haudenschild CC, Borst $C$. The impact of atheroselerotic arrerial remodeling on percentage of luminal stenosis varies widely within the arterial system. A postmortem study. Arterioster Thromb Var Biol. 1997:17:3057-3063.

12. Langille BL, Bendeck MP, Keeley FW. Adaptations of carotid arteries of young and mature rabbits to reduced carotid blood flow. Am J Pbysiol. 1989;256:1931-939.

13. Koller A, Sun D, Kaley G. Role of shear stress and endorhelial prostaglandins in flow-and wiscosity-induced dilation of arterioles in vitro. Cinc Res. 1993:72:1276-1284.

14. Berthiaume $\mathbb{E}$, Frangos JA. Flow-induced prostacyclin production is mediated by a pertussis toxin-sensitive G protein. FEBS Lett. 1992;308:277-279.

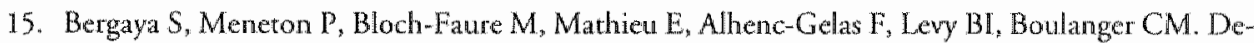
creased flow-dependent dilation in carorid arteries of tissue kallikrein-knockout mice. Cinc Res. 2001;88:593-599.

16. Huang A, Sun D, Carroll MA, Jiang H, Smith CJ, Connetra JA, Falck JR, Shesely EG, Koller A, Kaley G. EDHF nediates flow-induced dilation in skeleral muscle arterioles of female eNOS-KO mice. Am J Plysiol Heart Circ Plysiol. 2001;280:H2462-2469.

17. Barton M, Haudenschild CC, d'Uscio LV, Shaw S, Murike Ko Luscher TF. Endothelin ETA receptor blackade restores NO-mediated endorhelial function and inhibits atherosclerosis in apolipoprorein Edeficient mice. Proc Nat Acad Sci U S A 1998:95:14367-14372.

18. Bonthu S, Heistad DD, Chappell DA, Lamping KG, Faraci FM. Atherosclerosis, vascular remodeling, and impaiment of endohelium-dependent relaxation in genetically altered hyperlipidenic mice. Artertoucler Thromb Vasc Brol 1997;17:2333-2340.

19. Luscher TF. 1993 Mack Forster Award Lecture. Review. The endorhelium as a target and mediator of cardiowascular disease. Eur J Clin Innest. 1993;23:670-685.

20. Cooke IP. Does ADMA cause endothelial dysfunction? [I Process Citation]. Arteriocler 7hromb Vasc Biol 2000;20:2032-2037.

21. Pourageat $\mathrm{F}$, De Mey $1 \mathrm{G}$. Vasomotor responses in chronically hyperperfused and hypoperfused rat mesenteric arteries. Am J Physiol. 1998;274:H1301-1307.

22. Lamping KG, Nuno DW, Chappell DA, Faraci EM. Agonist-specific impaiment of coronary vascular function in genetically altered, hyperlipidemic mice. Am J Physiol. 1999;276:R1 1023-1029. 
23. Kanazawa K, Kawashima S, Mikami S, Miwa $\mathrm{Y}$, Hirata $K$, Suemarsu M, Hayashi $Y$, Itoh $H$, Yokoyama M. Endothelial constiturve nitric oxide synthase protein and mRNA increased in rabbit atheroselcroric aorta despine impaired endothelium-dependene vascular relaxation. Am J Pathol. 1996:148:1949-1956.

24. Minor RL, Jr., Myers PR, Guerra $\mathrm{R}_{2}$ Jr, Bates JN, Harrison DG. Diet-induced atheroselerosis increases the release of nitrogen oxides from rabbir aortal. J Clin Invest 1990;86:2109-2116.

25. Stroes E, Kastelein J, Cosentino F, Erkelens W, Wever R, Koomans H, Luscher T, Rabelink T. Tetrahydrobiopterin restores endothelial function in hypercholesterolemia. I CW In Inest. $1997,99: 4: 1-46$

26. Gupte $S A$, Okada $T$, Ochi R. Supcroxide and nitroglycerin srimulare release of PGF2 alpha and TrA2 in isolated tat heart. Am J Physiol. 1996;271:H2447-2453.

27. Wolin MS. Inreracrions of oxidants with vascular signaling systems. Anterioscler Thromb Vasc Biol. $2000: 20: 1430-1442$.

28. Bagi Z, Ungvan Z, Koller A Xanthine oxidase-derived reacrive oxygen spectes convert flow-induced arteriolar dilation ro constriction in hyperhomocysteinemia: possible role of peroxynitrite. Arteviousler Thromb Vasc Biol. 2002:22:28-33.

29. Pourageaud F, Freston JL. Impaired endothelial relaxacions induced by agonists and flow in sponraneously hypertensiwe rat compared to Wistar-Kyoto rat perfused coronary arteries. I Vaso Res. $1995 ; 32: 190-199$.

30. Paniagua OA, Bryant MB, Panza JA. Role of endothelial nitric oxide in shear stress-induced vasodilation of buman microwasculature: diminished activity in hypertensive and bypercholesterolemic patients. Circwlation. 2001;103:1752-1758.

31. Shiode N. Morishima N, Nakayama Kn Yamagata T, Matsuura H, Kajiyama G. Flow-mediated. vasodilation of human epicardial coronary arreries: effect of inhibitjon of nitric oxide synthesis. $J$ Am Coll Cardiol 1996;27:304-310.

32. Sun D, Huang A, Smith CI, Stackpole CJ, Connetra JA, Shesely EG, Koller A, Kaley G. Enhanced release of prostaglandins contributes to flow-induced arteriolar dilation in eNOS knockour mice. Cinc Res. 1999,85:288-293.

33. Wu $Y_{*}$ Huang A, Sun D. Falck JR, Koller A, Kaley G. Gender-specific compensation for the lack af $\mathrm{NO}$ in the mediarion of flow-induced arteriolar dilarion. Am / Physiol Hear Circ Physiol. $2001: 280: 1+2456-2461$.

34. Wang $C X, C a i l$, Wang PQ, Ouyang KQ, Wang YL, Xu SR. Shear-induced changes in endorhelin-1 secretion of microvascular endothelial cells. Microtrac Res. 2002;63:209-217.

35. Taurindo FR, Pedro Mde A, Barbeiro HV, Pileggi F, Carvalho MH, Augusto O, da Lux PL. Vascular free radicat release. Ex viwo and in vivo evidence for a flow-dependen endorhelial mechanism. Circ Res. 1994,74:700-709.

36. Kumar A, Hoover IL. Simunons CA, Lindner $V$, Shebuski RJ. Remodeling and neointimal formarion in the carorid artery of normal and P-selectin-deficient mice. Corculation. $1997966: 4333-4342$

37. Mondy IS, Lindner V, Miyashiro JK, Berk BC, Dean RH, Geary RL. Plareler-derived growth factor ligand and receptor expression in response to altered blood flow in vivo. Circ Res. $1997: 81: 320-327$. 
38. Bryant SR, Bjercke R], Erichsen DA, Rege A, Lindner V. Vascular remodeling in response to altered blood tow is mediated by fibroblast growh factor-2. Car Rex 1999;84323-328.

39. Harmon KJ, Couper LL, Lindner V. Strain-dependent vascular remodeling phenotypens in inbred mice. Am J Pathol 2000; 156:1741-1748.

40. Lurgens E, de Muinck ED, Heeneman S, Daemen MJ. Compensatory enlargement and stenosis develop in apoE(-1-) and apoE*3-Leiden transgenic mice. Arterioster Thromb Vaw Biol 2001:21: 1359-1365.

4. Barone FC, Knudsen DJ, Nelson AH, Fenerstein GZ, Willette RN. Mouse strain differences in susceptibility to cerebral ischemia are relared to cerebral wascular anatomy. I Cowb Blood Flow Metab. 1993;13:683-692.

42. Miyashiro JK, Poppa V, Berk BC. Flow-induced vascular remodeling in the rat carorid artery diminishes with age. Circ Res. 1997;81:311-319.

43. Henrion D, Terzi $\mathbb{E}$, Marrougui $K$, Duriez M, Boulanger $C M$, Colucci-Guyon E, Babinet $C$. Briand P. Friedlander G. Poirevin P. Levy $B$ I. Impaired flow-induced dilation in mesenteric resisrance arteries from mice lacking vimentin. / Clin Invest. 1997;100:2909-2914. 



\section{Effects of estrogen on the vascular injury response in estrogen receptor $\alpha, \beta$ (double) knockout mice}

Richard H. Karas, Henny Schulten, Gary Pare, Mark J. Aronovitz, Claes Ohlson, Jan-Ake Gustafsson, Micheal E. Mendelsohn 


\begin{abstract}
The two known estrogen receprors, ERa and ERA3, mediate the effects of estrogen in all targer tissues, including blood vessels. We have shown previously that estrogen inhibits vascular injury response to the same extent in female wild-type (WT), ERo knockout ( $\mathrm{E} R \alpha \mathrm{KO}_{\mathrm{CH}}$ ), and $\mathrm{ERB}$ knockout (ERRKOCH) mice. We generated mice harboring distuptions of both $\mathrm{ER} \alpha$ and $\mathrm{ER} B$ genes ( $\mathrm{ERO}, \mathrm{BKO} \mathrm{CH}_{\mathrm{H}}$ ) by breeding and studied the effect of $17 B$-estradiol $\left(\mathbb{E}_{2}\right)$ on vascular injury responses in ovariecromized female ER $\alpha, B K O_{\mathrm{CH}}$ mice and WT littemates. $\mathrm{E}_{2}$ inhbited increases in vascular medial area following injury in the WT mice but not in the ERo, $\$ \mathrm{BKOCH}$ mice, demonstrating for the first rime that the rwo known estrogen receptors are necessary and sufficient to mediate estrogen inhibition of a component of the wascular injury response. Surprisingly, as in WT lircermates, $\mathbb{E}_{2}$ still significantly increased urerine weight and inhibited vascular smooth muscle cell (VSMC) proliferation following injury in the $E R \alpha, B K O_{C H}$ mice. These data support rhat the role of estrogen receptors differs for specific components of the vascular injury response in the $\mathrm{ER} \alpha, 3 \mathrm{KO} \mathrm{CH}$ mice. The results leave unresolved whether $\mathbb{E}_{2}$ inhibirion of VSMC proliferation in $E R \alpha, B K O_{C H}$ mice is caused by a recepror-independent mechanism, an unidentified receptor responsive to estrogent, or residual activity of the ERC splice variant reported previously in the parenal $\mathbb{E} R \mathrm{KO} \mathrm{CH}$ mice. These possibilicies may be resolwed by studies of mice

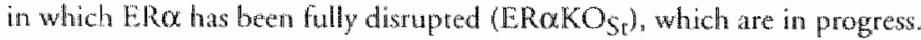




\section{INTRODUCTION}

The cardiovascular effects of estrogen are diverse. Estrogen has both systemic effects on circulating factors (eg, cholesterol, cytokines, coagulation/fibrinolytic factors) and direct effects on the blood vessel wall (eg, regulation of vasomotor tone, vascular cell proliferation; reviewed in Mendelsohn and Karas, ${ }^{1}$ Farhat et $\mathrm{al}^{2}{ }^{2}$ and Mendelsohn ${ }^{3}$ ) Some of the effects of estrogen occur rapidly, whereas others require prolonged estrogen exposure. At physiologically relevant concentrations of estrogen, both the rapid and the longer-term cardiovascular effects of estrogens are mediated by estrogen receptors. ${ }^{3-6}$ To date, two estrogen receptors (ERs) have been described, ER $\alpha$ and ERB (reviewed in Gustafsson [1997], ${ }^{7}$ Katzenellenbogen and Korach, ${ }^{8}$ and Gustafsson [1999] ${ }^{9}$ ). Although their physiological relevance in the vasculature is incompletely understood, ER $\alpha$ and ERß are expressed in endorhelial cells and vascular smooth muscle cells (VSMCs), ${ }^{10,11-16}$ the predominant cells present in vascular tissues. Using wild-type (WT) and estrogen receptor knockout (KO) mice, we have previously studied the role of ER $\alpha$ and ERß in mediating the vascular protective effects of estrogen in a mouse carotid artery injury model. ${ }^{10,17,18}$ Previous studies used mice developed at the University of North Carolina, Chapel Hill, which harbor gene deletions of either $\mathrm{ER} \alpha\left(\mathrm{ER} \alpha \mathrm{KO}_{\mathrm{CH}}\right)^{19}$ or $\mathrm{ERB}(\mathrm{ERBKO} \mathrm{CH}){ }^{20}$ These studies show that treatment of ovariectomized female mice with nanomolar concentrations of 17 -estradiol $\left(E_{2}\right)$ inhibits the response to vascular injury to equivalent levels in wild-type, ${ }^{17} \mathrm{ER} \alpha \mathrm{KO}_{\mathrm{CH}},{ }^{10}$ and $\mathrm{ER} \beta \mathrm{KO}_{\mathrm{CH}}$ mice. ${ }^{18}$ These findings suggest that $E R \alpha$ and $E R B$ are able to complement one another such that each receptor alone is sufficient to mediate the vascular protective effects of estrogen, or that the vascular protective effects of estrogen are mediated by an ER $\alpha /$ ERß-independent parhway. To distinguish berween these two hypotheses, we undertook the present study examining the effect of estrogen on the response to vascular injury in $\mathrm{ER} \alpha, B \mathrm{~K} \mathrm{O}_{\mathrm{CH}}$ (double) estrogen receptor knockout mice.

\section{MATERIAL AND METHODS}

\section{ANIMALS}

A total of over 820 animals were required to ultimately generate the $E R \alpha, B K \mathrm{O}_{\mathrm{CH}}$ mice used in this study. These mice, which have been extensively studied, do not express ER proteins in any tissue. ${ }^{19,20,21-23}$ However, ER $\alpha \mathrm{KO}_{\mathrm{CH}}$ mice have been shown previously to express mRNA for 2 partial ERo transcripts, one of which retains the hormone- and DNA-binding domains of full-length ER $\alpha$, and can both bind estradiol and mediate hormone-induced gene expression. ${ }^{23}$ This partial ERo transcript, detectable only by reverse transcriptase polymerase chain reaction approaches, may account for the low level of residual specific binding of estradiol in 
wterine tissue of the $\mathrm{ER} \alpha \mathrm{KO}_{\mathrm{CH}}$ mice ${ }^{23} \mathrm{ER} \alpha, \beta K \mathrm{O}_{\mathrm{CH}}$ mice were generared by extensive crossbreeding in one of our laboratories (C.O.) as follows: male and female double heterozygous $\left(\mathrm{ER} \alpha^{\left.+1-\beta^{+/}\right)}\right.$mice were created from the parental $E R \alpha K \mathrm{O}_{\mathrm{CH}}$ and ERRKO ${ }_{\mathrm{CH}}$ mice. Female offspring with the wild-rype ER $\alpha^{+/ 4} B^{+/ t}$ genorype and the double receptor knockout $E R \alpha^{-1-B^{-1}}$ genotype were included in the present study, ${ }^{19,20,24}$ and the latter are referred to as $\mathrm{ER} \alpha, \mathrm{BKO}_{\mathrm{CH}}$. More than 830 mice were required to generate a sufficient number of $\mathrm{ER} \alpha, \mathrm{BKO}_{\mathrm{CH}}$ female mice for the srudy. All mice were of mixed C57BL/6J/129 backgrounds. Genotyping of tail DNA was performed at 4 weeks of age and again at the end of the vascular injury experiment on tail snips. The ER $\alpha$ gene was analyzed with the following primer pairs. Primers AACTCGCCGGCTGCCACTTACCAT and CATCAGCGGGCTAGGCGAC $A C G$ for the WT gene correspond to flanking regions in the targeted exon 2 . They produce a fragment of 320 bp. Primers TGTGGCCGGCTGGGTGTG and GGCGCTGGGCTCGTTCTC for the knockout gene correspond to part of the Neo cassette and the flanking exon 2 . They produce a 700 -bp fragment. Genotyping of the ERß gene has been described previously. ${ }^{25}$ (The primers used for the ERß gene were one primer in intron 2 [BNHD4-25; 5'-AGAATGTTGCACTG- CCCC TGCTGC-3'], one in inron 3 [Clwt-27; 5'-GGAGTAGAA- ACAAGCAA. TCCAGACATC-3'], and one in the Neo cassette [Neo-25; 5'-GCAGCCTCTGT'T CCACATACACTTC-3']. A 650-bp product [BNHD4-25 and C1wt-27] was amplified for the homozygous wild-type [WT; $+/+]$ mice, and a 450-bp [BNHD4-25 and Neo-25] product was amplified for the homozygous mutant [-1-] mice; both bands were amplified for the heterozygous [+/-] mice). Animals had free access to fresh water and food pellets.

\section{CAROTID ARTERY INJURY}

All procedures and protocols were approved by the New England Medical Center Animal Research Committee. A detailed description of the vascular injury protocol has been published previously. ${ }^{10,17,18}$ Briefly, 7 days following ovariectomy, 26 wild-type and $23 \mathbb{E R} \alpha, \beta K \mathrm{KO}_{\mathrm{CH}}$ female mice, average age 12 to 13 weeks, were randomized to receive either placebo pellets $\left(-\mathbb{E}_{2}\right)$ or 178 -estradiol-containing pellets $\left(+\mathrm{E}_{2} ; 0.1 \mathrm{mg} / 21\right.$-day pellets, Innovative Research) at a dose previously shown to produce physiologically rellevant (ie, low nanomolar) concentrations of circulating 178 -estradiol $\left(\mathrm{E}_{2}\right)$. A week after pellets were implanted, the mice were anesthertized with inhaled isoflurane and subjected to unilareral vascular injury by intraluminal passage of a wire into the left common carorid artery resulting in endothelial denudation. At this time, osmotic minipumps calibrated to release bromodeoxyuridine (BrdU) over the 14 days of the experiment were implanted subcutaneously, as described. 17 The mice were allowed to recover and return to normal activity. 
Two weeks following vascular injury, the mice were anesthetized with $2.5 \%$ isoflurane and blood was taken for cholesterol and estradiol determinations. Uteri where then extracted and wer weights obtained, followed by harvesting of a small segment of the tail for confirmation of pre-study genotype analysis by reverse transcriptase- polymerase chain reaction. Both carorid arteries were harvested next after perfusion fixarion with $10 \%$ formalin at $150 \mathrm{mmHg}$ for 4 minutes. A small segment of small intestine was also harvested for use as a positive control for the BrdU stains (see next section).

\section{IMMUNOHISTOCHEMISTRY AND MORPHOMETRY}

Following embedding in paraffin, parallel sections from all 98 carotid arteries were stained as previously described for hematoxylin/eosin and for elastin. $10,17,18,26$ Immunodetection of BrdU-labeled cells was also performed as previously described. ${ }^{10,17,18}$ A section of small intestine was included on each slide to serve as a positive control for the BrdU stain. One section of each vessel was not exposed to a primary antibody, serving as a negative control. VSMCs were identified by immunostaining for smooth muscle specific actin, performed on a Vantana automated slide staining machine using clone 1A4 from Sigma as the primary antibody. BrdU-stained VSMCs and unstained VSMC nuclei were counted by two independent observers. A VSMC proliferation index also was calculated by dividing the number of BrdU-labeled VSMCs by the total number of unstained VSMCs for each section. Medial area was determined on serial elastin-stained sections using the ImagePro Plus software. All observations were made by observers blinded to both the genotype and the treatment group of the specimen. Only one vessel developed an occlusive thrombosis, and this mouse from the WT- $\mathrm{E}_{2}$ group was excluded from analysis.

\section{ADDITIONAL ASSAYS}

Circulating concentrations of $E_{2}$ were measured by radioimmunoassay using a commercially available kir (Ultra-Sensitive Estradiol RLA, Diagnostic System Laboratories Inc), according to the manufacturer's instructions. Pooled samples of serum $(200 \mu \mathrm{L})$ from each of the treatment groups were each assayed in triplicate, and the mean value is reported as representative of the group. Control experiments confirmed the linearity of the assay between the range of 0.018 and $2.78 \mathrm{nmol} / \mathrm{L}$. Circulating concentrations of total and HDL cholesterol, and triglycerides were determined as previously described. ${ }^{17}$ At the time of euthanasia, the correctgenotype of each mouse was confirmed by a second independent set of PCR studies using tail-snip DNA as described above. 


\section{STATISTYCAL ANALYSIS}

For all analyses, differences between the 4 treatment groups were first examined with a one-way ANOVA after the normality of the data were tested. Post hoc comparisons were made with the Student-Newman-Keuls test. Two group comparisons were made with the Student's t-test. All statistics were performed using SigmaStat software. A value of $\mathrm{P}<0.05$ was considered significant.

\section{RESULTS}

As in our previous studies, implantation of $\mathrm{E}_{2}$-containing pellets in the ovariectomized female mice resrored circulating levels of $\mathrm{E}_{2}$ to physiological levels in borh the WT and $E R \alpha, B K O_{\mathrm{CH}}$ mice $(0.43 \pm 0.03 \mathrm{nmol} / \mathrm{L}[115 \pm 9 \mathrm{pg} / \mathrm{mL}]$ and $0.55 \pm 0.03 \mathrm{nmol} / \mathrm{L}[148 \pm 7 \mathrm{pg} / \mathrm{mL}]$, respectively; Table1). There were no significant differences in body weight, blood pressure, or heart rate at baseline or ar the rime of injury between any of the groups (Table1). Cholesterol levels also were similar between the WT and $E R \alpha, B K_{C H}$ animals, and not significantly changed by estrogen treatment (Table1). In the W'T animals, $\mathrm{E}_{2}$ treatment resulted in the expected approximately 10 -fold increase in uterine weight compared with the $-\mathrm{E}_{2}$ group (Figure 1; $\mathrm{P}<0.001 \mathrm{WT}+\mathrm{E}_{2}$ versus $\left.\mathrm{W} T-\mathrm{E}_{2}\right)$. The uterine weights were lower in the placebo-treated $\mathrm{ER} \alpha, \beta \mathrm{KO}_{\mathrm{CH}}$ mice than in the placebo-treated WT mice $\left(\mathrm{P}<0.01 \mathrm{ER} \alpha, B \mathrm{KO}_{\mathrm{CH}}-\mathrm{E}_{2}\right.$ versus $\left.\mathrm{WT}-\mathrm{E}_{2}\right)$. Surprisingly, $\mathrm{E}_{2}$ replacement to physiological levels also significantly increased uterine weight in the $\mathrm{ER} \alpha, \mathrm{BKO}_{\mathrm{CH}}$ mice (5.8-fold; Figure 1; $\mathrm{P}<0.01 \mathrm{ER} \alpha, \beta \mathrm{KO}_{\mathrm{CH}}+\mathrm{E}_{2}$ versus $\mathrm{ER} \alpha, \mathrm{BKO}_{\mathrm{CH}}-\mathrm{E}_{2}$ ), although to a lesser extent than in the WT mice. Uterine weight increased in every animal assigned to receive $\mathrm{E}_{2}$-containing pellets, confirming proper placement and function of the pellets. The 2 primary endpoints for the mouse carotid artery injury model are well established $10,17,18$ and include (1) change in medial area and (2) extent of VSMC proliferation, both assessed 14 days after unilateral endothelial denudation injury. The medial-area endpoint reflects the combined effects of alterations in extracellular matrix constiruents and cellular volume, whereas the VSMC proliferation endpoint specifically indicates the degree of mitogenesis that occurs in these cells in response to the vascular injury. 
Table 1. Effects of $\mathbb{E}_{2}$ on Wild-Type and ERO,BROMH Mice

\begin{tabular}{|c|c|c|c|c|}
\hline & \multicolumn{2}{|c|}{ Wild Type } & \multicolumn{2}{|c|}{$\mathrm{ERa}_{0} \beta \mathrm{KO}_{\mathrm{CH}}$} \\
\hline & $-\mathrm{E}_{2}$ & $+\mathrm{E}_{2}$ & $-\mathbb{E}_{2}$ & $+E_{2}$ \\
\hline E level, nmol/L & $<0.02$ & $0.43 \pm 0.03$ & $<0.02$ & $0.55 \pm 0.03$ \\
\hline Body weight at injury, g & $23.3 \pm 0.5$ & $24.4 \pm 0.4$ & $24.4 \pm 0.5$ & $25.1 \pm 0.7$ \\
\hline Body weight at sacrifice, g & $24.7 \pm 0.4$ & $24.9 \pm 0.3$ & $25.6 \pm 0.6$ & $26,1 \pm 0.5$ \\
\hline Tail-cuff blood pressure, $\mathrm{mmHg}$ & $105 \pm 2.1$ & $100 \pm 0.5$ & $100+5.2$ & $98 \pm 3.5$ \\
\hline Heart rate, bpm & $633 \pm 37$ & $586 \pm 38$ & $693 \pm 11$ & $651 \pm 24$ \\
\hline Total cholesterol, mg/dL & 78 & 68 & 79 & 76 \\
\hline Triglycerides, $\mathrm{ng} / \mathrm{dL}$ & 167 & 211 & 187 & 199 \\
\hline HDL cholesterol, mg/dL & 66 & 60 & 69 & $6 ?$ \\
\hline
\end{tabular}

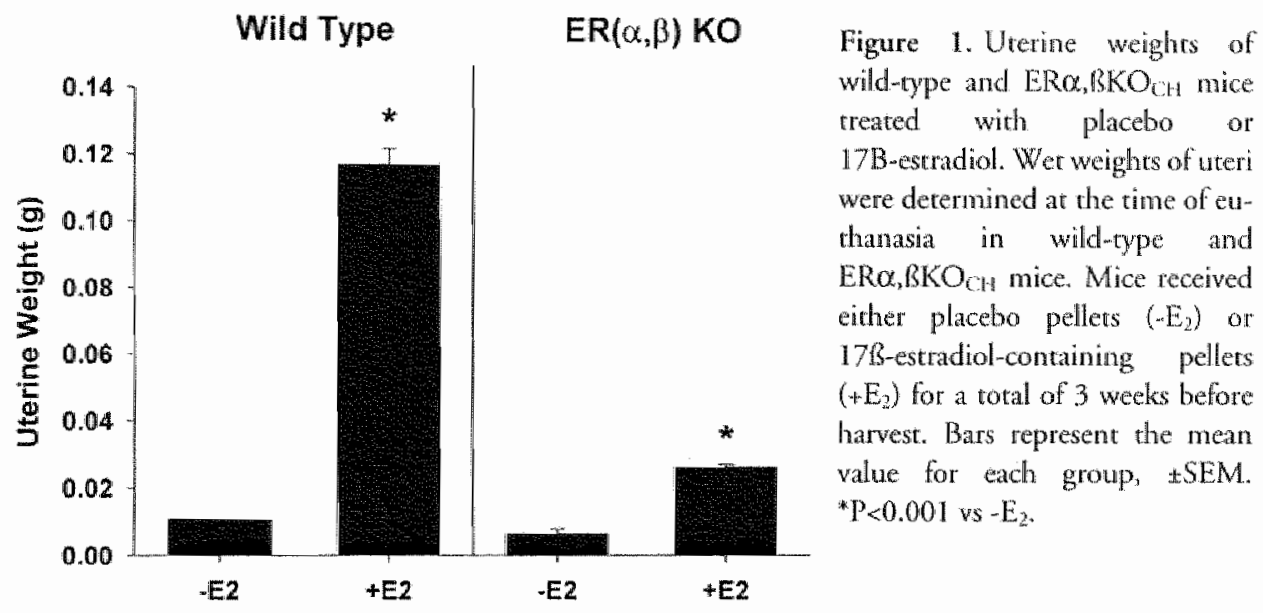

RESPONSE TO VASCULAR INJURY: MEDIAL AREA

Medial areas were determined by computerized morphometric analysis of elastin-stained sections of each carotid artery. A summary of the medial area results is presented in Figure 2 . The medial areas of the uninjured carotid arteries were similar in the WT $(\mathrm{n}=26)$ and $\mathbb{E} R \alpha, B K \mathrm{BO}_{\mathrm{CH}}(\mathrm{n}=23)$ mice $\left(15.5 \pm 0.3 \times 10^{-3} \mathrm{~mm}^{2}\right.$ versus $15.3 \pm 0.5 \times 10^{-3} \mathrm{~mm}^{2}$, respectively; $\mathrm{P}=\mathrm{NS}$ ). In the placebo-treated WT mice, injury induced an increase in the medial area to $21.3 \pm 1.6 \times 10^{-3} \mathrm{~mm}^{2}\left(\mathrm{P}<0.01\right.$ WT- $\mathrm{E}_{2}$ versus uninjured). Estrogen replacement in the WT mice significantly inhibited the injury-induced increase in medial area, resulting in a medial area of $16.0 \pm 1.4 \times 10^{-3}$ $\mathrm{mm}^{2}\left(\mathrm{P}=0.03 \mathrm{WT}+\mathrm{E}_{2}\right.$ versus $\left.\mathrm{WT}-\mathrm{E}_{2}\right)$. The medial area of the injured carotid in the $W T+E_{2}$ animals was indistinguishable from that of the uninjured contralateral vessel $\left(\mathrm{P}=0.7 \mathrm{WT}+\mathrm{E}_{2}\right.$ versus uninjured). In the placebo-treated $\mathrm{ER} \alpha, B K \mathrm{O}_{\mathrm{CH}}$ mice, injury 

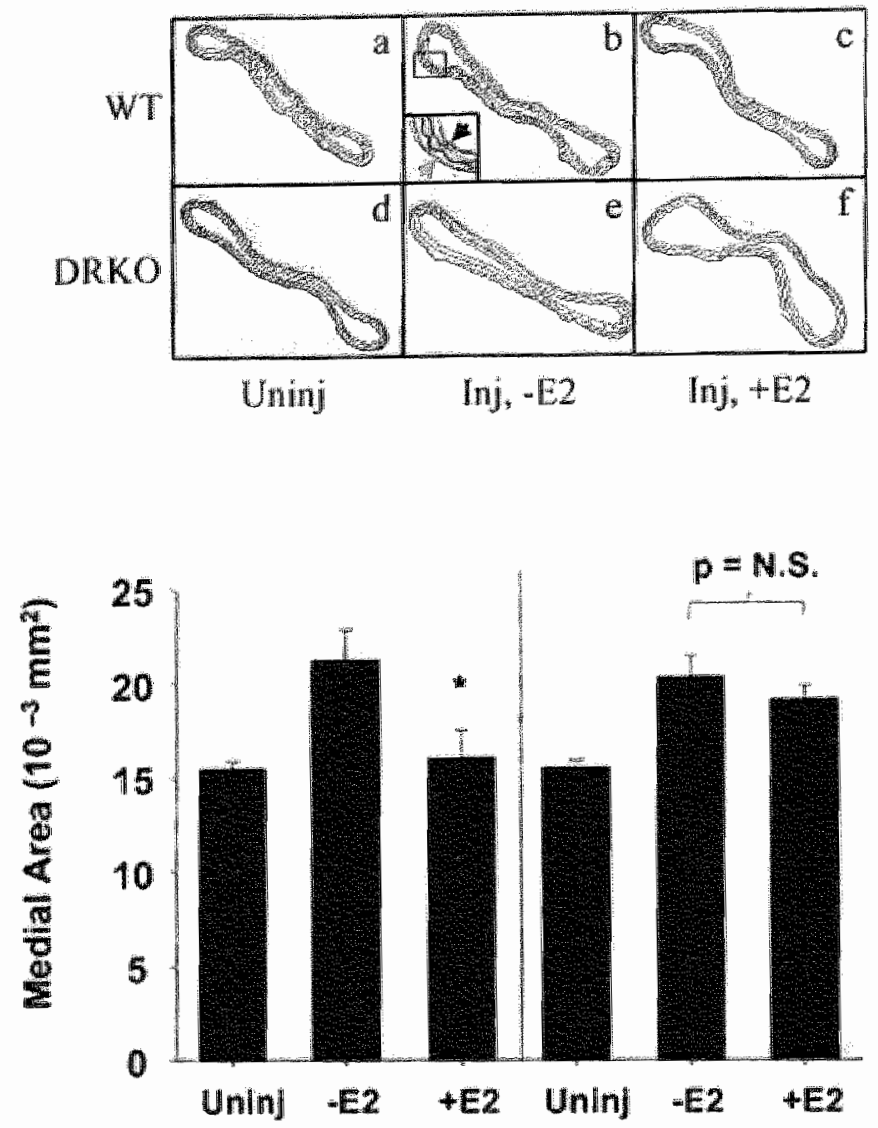

Figure 2. Medial areas of uninjured and injured carotid arteries from wild-type and ERO,BKO an mice treated with vehicle or 17R-estradiol. a through $f$. Representarive dastin-stained sections from uninjured (Uninj) and injured (Ini) carotid arteries from wild-type (WT) and $\mathrm{ERO}, \mathrm{BKO}_{\mathrm{CH}}$ mice rreated with placebo pellets $\left(-E_{2}\right)$, or 178 -estradiol-containing pellets $\left(+E_{y}\right)$ ate shown $(x 400)$. The medial area of each specific section shown is listed in parentheses. The medial area was determined on the entire secrion, a, Wild-rype; uninjured $\left(15.5 \times 10^{-3} \mathrm{~mm}^{2}\right)$. b, Wild-rype, injured, placebo-rreated $\left(22.2 \times 10^{4} \mathrm{~mm}^{2}\right)$. c. Wild-rype, injured, E-treated $\left(15.4 \times 10^{-3}\right.$ $\left.\mathrm{mm}^{2}\right)$. d, ERQ, $B \mathrm{KO}_{\mathrm{CH}}$, uninjured $\left(14.7 \times 10^{-3} \mathrm{~mm}^{2}\right)$, e, ERo, (sKOCH, injured, placebo-treated $\left(21.6 \times 10^{3} \mathrm{~mm}^{2}\right)$. $\mathrm{FRQ}, B \mathrm{KO}_{\mathrm{CH}}$, injured, $\mathrm{E}_{2}$-treared $\left(18.9 \times 10^{-3} \mathrm{~mm}^{2}\right) . \mathrm{g}$, Sumnary of medial area resules for all mice. In panel $b$, the external elastic lamina is identifed by the red arrow, and the internal dastic lamina is identified by the black arrow. In paryel g, bars represent the mean walue for each group, $\pm S E M$. ${ }^{2}<0.05$ ws the inj, $-E_{2}$ group within the same genorype. 

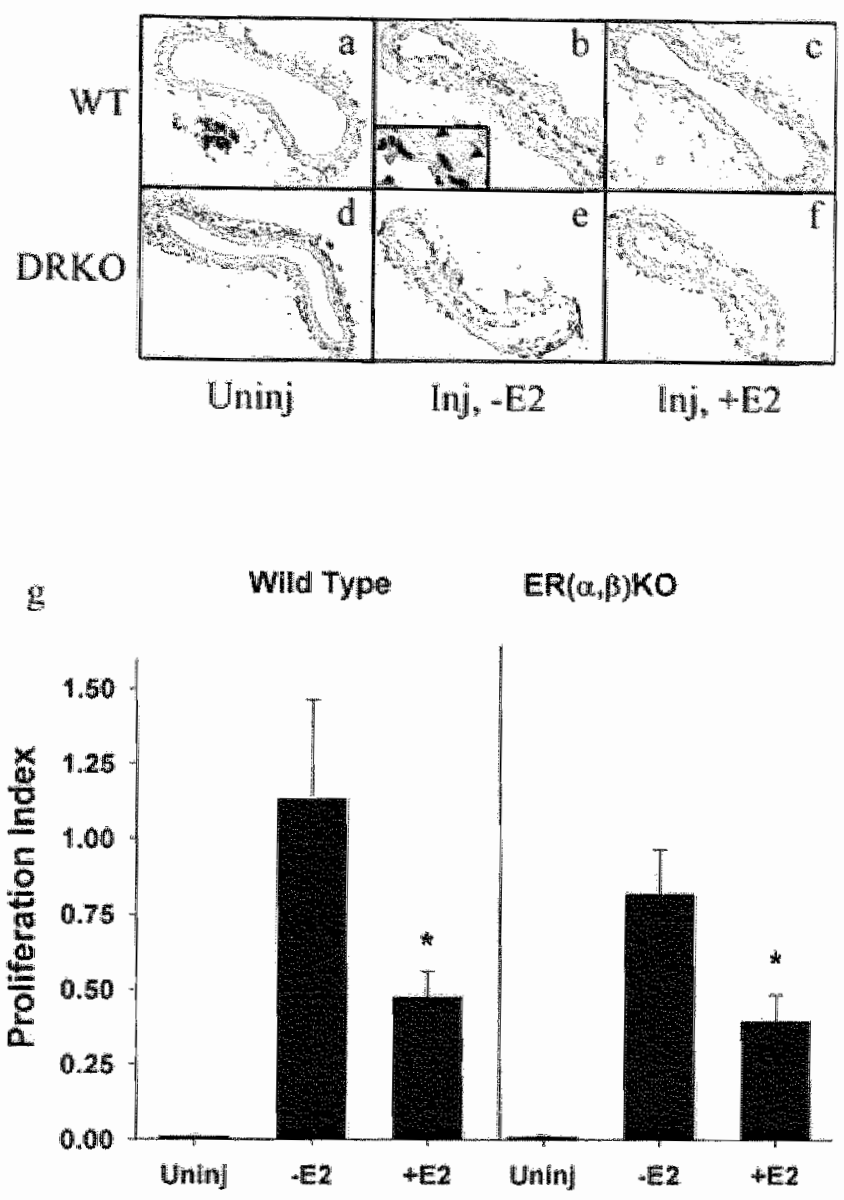

Figure 3. Vascular smootl muscle cell proliferation of uninjured and injured carrotid arteries from wild-type and ERO, $B K O$, mice treated with wehicle or 17R-estradiol. a through $\mathrm{F}_{2}$ Representative BrdU-stained secrions from uninjured (Uninj) and injured (Inj) carotid arteries from wild-type (WT) and ERa,BKO mice reated with placebo pellens (-E $E_{2}$ or 17 B-estradiol-containing pellets $\left(4 \mathrm{E}_{2}\right)$ are shown ( $\left.\mathrm{x} 400\right)$. The VSMC proliferation index (BrdU-labeled VSMCs/unstained VSMCs) of each specific secrion shown is listed in parentheses. The VSMC proliferation index was determined on the entire section. $a$, Wild-rype, uninjured. $b$, Wild-type injured, placebo-treated $(0.90)$, $c_{n}$ Wild-type, injured, E $z^{-t r e a t e d}(0.54)$. d,

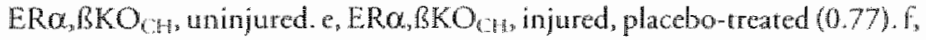

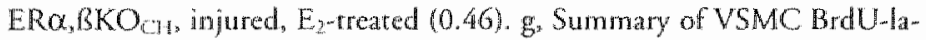
beling resuls for all mice. In panel $g$, bars represent the mean walue for each group, mean $\mathrm{SEM}$. "P<0.05 vs the Inj, - E, group within the same genorype. 
induced a significant increase in medial area $\left(20.3 \pm 1.1 \times 10^{-3} \mathrm{~mm}^{2} ; \quad P<0.01\right.$ ERO, $B K_{\mathrm{CH}}-\mathrm{E}_{2}$ versus uninjured), similar to the degree of injury response in the placebo-rreated WT mice $\left(\mathrm{P}=0.6 \mathrm{ER \alpha}, \mathrm{BKO}_{\mathrm{CH}}-\mathrm{E}_{2}\right.$ versus WT-E $)$. In contrast to the findings in the WT mice, in the $E R \alpha, B K \mathrm{KO}_{\mathrm{CH}}$ mice $\mathrm{E}_{2}$ treatment did not significantly inhibit the injury-induced increase in medial area $\left(19.0 \pm 0.7 \times 10^{-3} \mathrm{~mm}^{2}\right.$ in the $\mathrm{ER} \alpha_{3}, \mathrm{BKO}_{\mathrm{CH}}+\mathrm{E}_{2}, \mathrm{P}=0.5$ wersus $\left.\mathrm{ER} \alpha, \mathrm{BKO}_{\mathrm{CH}}-\mathrm{E}_{2}\right)$. Thus, the injury-induced increase in medial area was similar in the placebo-treated WT and $\mathrm{ER} \alpha, \beta \mathrm{KO}_{\mathrm{CH}}$ mice, but $\mathrm{E}_{2}$ replacement no longer inhibited the increase in medial area following injury in the $\mathrm{ER} \alpha, \beta \mathrm{KO}_{\mathrm{CH}}$ mice. In contrast to any of our previous studies using this model, in over 300 animals (References 10, 17, 18, and unpublished results, 2000-2001), these results in the $\mathrm{ER \alpha}, \mathrm{BKO}_{\mathrm{CH}}$ mice represent the first instance that $\mathrm{E}_{2}$ has falled to protect against an endpoint analyzed to quantify the vascular response to carotid injury.

\section{RESPONSE TO VASCULAR INJURY: VSMC PROLIFERATION}

VSMC proliferation was assessed by immunohistochemical detection of BrdU-labeled VSMCs in sections from each carotid artery. A summary of these results is presented in Figure 3. There were no differences in the morphological appearance or total number of nuclei in the uninjured vessels of the WT and $\mathrm{ER} \alpha, B K \mathrm{KO}_{\mathrm{CH}}$ mice $(95 \pm 15$ versus $93 \pm 14$ cells/secrion, respectively; $\mathrm{P}=\mathrm{NS}$ ). This supports that there are no obvious developmental abnormalities in the formation of the vasculature in the ERo, BKO $\mathrm{CH}_{\mathrm{H}}$ mice. As expected, BrdU-labeled VSMCs were only rarely detected in the uninjured vessels of either the WT or the $\mathrm{ER} \alpha, B \mathrm{KO} \mathrm{CH}_{\mathrm{CH}}$ mice (mean $<1$ labeled cell per vessel for $\mathrm{WT}$ and $\mathrm{ER} \alpha, \mathrm{BKO}_{\mathrm{CH}}$ groups; $\mathrm{P}=\mathrm{NS}$ between groups). As in our previous studies using this injury rechnique, a small amount of neointima was observed in a minority of injured vessels and there were no significant differences in neointimal formation in the WT compared with the $E R \alpha, B K O_{C H}$ mice (data not shown). In the placebo-treated WT mice, injury increased the VSMC proliferation index from $<0.1$ to $1.1 \pm 0.3$ ( $P<0.001$ WT- $E_{2}$ wersus uninjured). This increase in VSMC proliferation was significantly atrenuated. by $\mathrm{E}_{2}$ treatment in the WT mice, resulting in a VSMC proliferation index of $0.5 \pm 0.1$ $\left(\mathrm{P}<0.05 \mathrm{WT}+\mathrm{E}_{2}\right.$ versus $\left.\mathrm{WT}-\mathrm{E}_{2}\right)$. In the placebo-treated $\mathrm{ER} \alpha, \beta \mathrm{KO}_{\mathrm{CH}}$ mice, injury also induced an increase in the VSMC proliferation index from $<0.1$ to $0.8 \pm 0.1$ $\left(\mathrm{P}<0.001 \mathrm{ER} \alpha, \beta \mathrm{KO}_{\mathrm{CH}}-\mathrm{E}_{2}\right.$ versus uninjured). The injury-induced increase in VSMC proliferation in the placebo-treated $\mathrm{ER} \alpha, \mathrm{BKO}_{\mathrm{CH}}$ mice was similar to that observed in the placebo-treated WT mice $\left(\mathrm{P}=0.4 \mathrm{ER} \alpha, B \mathrm{KO}_{\mathrm{CH}}-\mathrm{E}_{2}\right.$ versus WT- $\left.\mathrm{E}_{2}\right)$. As in the WT mice, $\mathrm{E}_{2}$ treatment of the $\mathrm{ER} \alpha, \beta \mathrm{KO}_{\mathrm{CH}}$ mice significantly attenuated the injury-induced increase in the VSMC proliferation index $(0.4 \pm 0.1 ; \mathrm{P}<0.05$ ERo,, $3 \mathrm{KO}_{\mathrm{CH}}+\mathrm{E}_{2}$ versus $\mathrm{ER} \alpha, \beta \mathrm{KO}_{\mathrm{CH}}-\mathrm{E}_{2} ; \mathrm{P}=0.6 \mathrm{ER} \alpha, \beta K \mathrm{KO}_{\mathrm{CH}}+\mathrm{E}_{2}$ versus WT $+\mathrm{E}_{2}$ ). Thus, injury induced an increase in VSMC proliferation to similar degrees in the placebo-treated WT and $\mathrm{ER} \alpha, \mathrm{BKO}_{\mathrm{CH}}$ mice, and the inhibitory effect of $\mathrm{E}_{2}$ on the 
injury-induced increase in VSMCs observed in the WT animals was retained in the $\mathrm{ER} \alpha, \beta \mathrm{KO}_{\mathrm{CH}}$ mice. These findings demonstrate maintenance of the protective effect of $\mathrm{E}_{2}$ on VSMC proliferation in the ERaKOCH mice, in surprising contrast to the loss of the protective effect of $E_{2}$ on the medial-area endpoint presented above. Recent data demonstrate clearly that estrogen receptors mediare both genomic and nongenomic effects in vascular cells ar physiological levels of estrogen. ${ }^{3-6}$ ER $\alpha$ was the first and only known estrogen receptor from 1985 , when it was inirially cloned,27 until 1996, when ERß was discovered. ${ }^{28-30}$ Although ERo and ERB are the products of distinct genes, they share relatively high degrees of homology in the DNA binding domain $(\approx 95 \%)$ and in the ligand binding domain $(\approx 55 \%)$. Recent studies have begun to define receptor-specific differences in ligand binding affinity, $28,31-33$ receptor interacting proteins, ${ }^{34,35}$ and pharmacologic agonists and antagonists. ${ }^{36-38}$ These findings highlight the potential for differences in the physiological effects of these two receptors. In 1995, we first reported that estrogen inhibits the vascular injury response in normolipemic wild-type mice, ${ }_{2}^{17}$ as it does in orher animal models (reviewed in Mendelsohn and Karas'). A subsequent study showed equivalent inhibition of the vascular injury response in the ER $\alpha \mathrm{KO}_{\mathrm{CH}}$ mice created by Lubahn et al ${ }^{19}$ compared with their littermate wild-type controls. ${ }^{10}$ A more recent study in which $\mathrm{ER} \alpha \mathrm{KO}_{\mathrm{CH}}$ mice were bred with the hyperlipidemic apolipoprotein $\mathrm{E}$ knockout mice demonstrated that in this model estrogen no longer decreased plaque size, but continued to significantly alter plaque complexity, decreasing the number of lesions with fibrous caps, calcifications, and cholesterol clefts. ${ }^{39}$ These studies support the existence of $E R \alpha$-independent vascular protective effects of $E_{2}$ or of residual $E R \alpha$ activity in the $E R \alpha K O_{\mathrm{CH}}$ mice. ${ }^{23}$ The discovery of $E R B,{ }^{28}$ identification of its expression in vascular cells and tissues ${ }_{3}{ }^{10}$ and demonstration that vascular injury dramatically enhances vascular cell expression of ERß but not $E R \alpha^{13,16}$ all raised expectations that ERB might mediate the vascular protective effects of estrogen. However, a subsequent carotid artery injury study in the ERßKO mice demonstrated that $E_{2}$ continued to inhibit the response to injury equally well in ERRKO and WT mice. ${ }^{18}$ These studies, which used the only available ER knockout mice at that time, ${ }^{1920}$ suggested that neither ERo nor ERß by themselves are necessary for the protective effect of estrogen in the vasculature. In the present study, the effect of $\mathrm{E}_{2}$ on the usual two vascular injury endpoints, medial area and VSMC proliferation, was compared in $E R \alpha, B K O_{\mathrm{CH}}$ and WT mice. In the willd-type mice, $\mathrm{E}_{2}$ inhibited the injury-induced increase in medial area as it has in each of our previous studies. ${ }^{10,17,18}$ In contrast, in the $\mathrm{ER} \alpha, B \mathrm{BKO} \mathrm{CH}_{\mathrm{H}}$ mice, $\mathrm{E}_{2}$ did not prevent the injury-induced increase in medial area. Loss of the protective effect of estrogen on the medial-area endpoint in the $\mathrm{ER} \alpha, B \mathrm{KO}_{\mathrm{CH}}$ mice supports that $\mathrm{ER} \alpha$ and $\mathrm{ER} R$ mediate this effect of estrogen. Thus, for the first time, ER $\alpha$ and ERß are implicated as physiologically relevant mediators of one component of the vascular injury response. Taken together with the results of previous single estrogen receptor knockout experiments, ${ }^{10,18}$ these data support that the ERO and ERB can functionally complement 
one another in vivo, such that either receptor alone can mediate the inhibirory effect of $\mathrm{E}_{2}$ on the medial area thickening endpoint (see subsequent caveat). The $\mathrm{E}_{2}$-mediated inhibition of VSMC proliferation observed in the WT mice in the present study also is consistent with our previous observations in the WT mice. ${ }^{10,17,18}$ Quite surprisingly, however, estrogen also inhibited VSMC proliferation in the $E R \alpha, \beta K \mathrm{KO}_{\mathrm{CH}}$ mice to a similar extent as in the WT mice. One interpretation of the preserved estrogenic effect on VSMC proliferation in ER $\alpha, 3 K \mathrm{KO}_{\mathrm{CH}}$ mice is the exciting possibility that there are estrogen receptors other than ER $\alpha$ and ERB that have not yet been identified. However, to date, extensive searching has failed to idenrify any likely candidates (authors' unpublished results, 1997-2001). It is important to note that $\mathrm{E}_{2}$-mediated inhibition of VSMC proliferation was not the only estrogenic effect observed in the $\mathrm{ER} \alpha, \mathrm{BKO}_{\mathrm{CH}}$ mice. Estrogen exposure also resulted in a significant increase in $\mathrm{ER} \alpha, \mathrm{BKO}_{\mathrm{CH}}$ uterine weights (Figure 1). This demonstrates that preservation of an effect of estrogen in the $\mathrm{ER} \alpha, B \mathrm{KO} \mathrm{CH}_{\mathrm{CH}}$ mice is not confined to, or specific for, the cardiovascular system. The estrogen responsiveness of the uterus in these mice raises an additional consideration. The $E R \alpha, B K O_{C H}$ mice used in the present study were produced by breeding $\mathrm{ER} \alpha \mathrm{KO}_{\mathrm{CH}}$ and ERBKO $\mathrm{CH}_{\mathrm{H}}$ mice. 19,20,21,22 Uteri of ER $\alpha \mathrm{KO}_{\mathrm{CH}}$ mice express mRNA for 2 partial ER $\alpha$ transcripts. ${ }^{23}$ Although one of these transcripts is nonfunctional, the other transcript, E1, is a splice variant of the wild-type ER $\alpha$ in which only a portion of exon 2 is omitted. 23 The protein encoded by this mutant sequence appears to bind estrogen wich an affinity similar to that of the wild-type receptor, and to be capable of mediating hormone-induced. gene expression. ${ }^{23}$ In this prior study, immunoblot analyses of $E R \alpha \mathrm{KO}_{\mathrm{CH}}$ uterus did not detect expression of a protein from the $\mathrm{E} 1$ splice variant. ${ }^{23}$ However, it remains quite possible that the estrogenic effects observed on VSMC proliferation and uterine weight in the present study are mediated by expression of low levels of this mutant ER $\alpha$. If the E1 transcript mediates the effects of estrogen on uterine weight and/or VSMC proliferation in the $\mathrm{ER} \alpha, B \mathrm{KO}_{\mathrm{CH}}$ mice (and the parental $\mathrm{ER} \alpha \mathrm{KO}_{\mathrm{CH}}$ mice ${ }^{10}$ ), this would have widespread implications for the published reports that have used $\mathrm{ER} \propto \mathrm{KO}_{\mathrm{CH}}$ mice to study various physiological systems (reviewed in Couse and Korach ${ }^{40}$ ). The mechanism by which estrogen continues to inhibit VSMC proliferation and increase uterine weight in the $\mathrm{ERa}, \mathrm{BKO}_{\mathrm{CH}}$ mice thus remains unclear. Receptor-independent effects of estradiol could be involved, although there is little physiological evidence in support of this explanation. 'The presence of an unidentified, novel estrogen receptor could also account for the persistent ability of $\mathbb{E}_{2}$ to inhibir VSMC proliferation, although no $\mathrm{ER} \alpha$ has been identified despite extensive searching. However, another member of the steroid hormone receptor superfamily ${ }^{41}$ (eg, ERR $\alpha, E R R \beta, E R R \alpha$, or an orphan receptor) with the ability to respond to estrogen could be involved. Alternatively, a low level of expression of the El estrogen receptor splice variant identified by Couse et $\mathrm{al}^{23}$ in the $\mathrm{ER} \alpha \mathrm{KO}_{\mathrm{CH}}$ parental strain could mediate residual estrogen responsiveness in $E R \alpha, B K O_{C H}$ mice. The explanation for persistent effects of estrogen in this study may be resolved by our ongoing 
studies of the effect of $E_{2}$ on the vascular injury response in mice recently created in the Chambon laboratory in Strasbourg, France (ER $\left.\alpha K_{S O}\right), 42$ in which ER $\alpha$ has been fully disrupted. Understanding the mechanism by which estrogen responsiveness is retained in the $E R \alpha, B K O_{C H}$ mice has important implications for our understanding of estrogen receptor-mediated signaling in all target tissues, including the cardiovascular system.

\section{ACKNOWLEGDMENTS}

This work was supported in part by NIH P50 HL63494, NIH R01 HL55309, and NIH R01 HL56069 (M.E.M.); NIH R01 HL61298 (R.H.K.); the Swedistn Medical Research Council and the Swedish Foundarion for Strategic Research (C.O.); and by KaraBio $A B$ and the Swedish Cancer Fund (J.-A.G.). The authors would like to thank Kristen Whitehead and Sharon Lynch for expert preparation of this manuscript. Its contents are solely the responsibility of the authors and do nor necessarily represent the official views of $\mathrm{NIH}$.

\section{REFERENCES}

1. Mendelsohn ME, Karas RH. Mechanisms of disease: the protective effecs of estrogen on the cardiovascular system. N Engl/ Med. 1999; 340: 1801-1811.

2. Farhat MY, Lawigne MC, Ramwell PW. The vascular protective effects of ecrogen. FASEB I. $1996 ; 10: 615-624.3$.

3. Mendelsohn ME. Nongenomic, ER-mediated activation of endothelial nitric oxide synthase: how does it work? What does it mean? Circ Res. 2000; 87: 956-9603.

4. Chen Z, Yuhanu IS, Gallcheva-Gargova ZI, Karas RH, Mendelsohn ME, Shaul PW. Fstrogen receptor $\alpha$ mediates the nongenomic activation of endothellat nitric oxide synthase by estroger. J Chin Invest. 199\%: 103: 401-406.

5. Haynes MP', Sinha D, Strong Russell K. Collinge M, Fulton D. Morales-Ruiz M. Bender JR. Membrane estrogen recepror engagement activates condothelial nicric oxide symthase via tho P13-kinase-Akr parhway in human endohelial cells. Cir Res. 2000; 87:677-682.

6. Simoncini T, Hafezi-Moghadam A, Brazil DP, Ley $\mathbb{K}$, Chin WW, Lia JK. Interaction of oesrogen recepror with rhe regularory subunit of phosphatidylinosirol-3-OH kinase. Nature $2000 ; 407$ : $538-541$.

7. Gustafsson 1 A. Estrogen receptor $\mathbb{B}$ tgerting in on the acrion? Nat Med. 1997: 3: $493-494$.

8. Karzenellenbogen BS, Korach KS. A new actor in the estrogen receptor drama: enter ER-B. Endow crinotagy. 1997: 1381:861-862.

9. Gustafsson $J A$. Estrogen receptor $B$ : a new dimension in estrogen mechanism of action. I Endocrinol. 1909; 163: 379-383. 
10. Iafrati MD, Katas RH, Aronowiz M. Kum S, Sulliwan TR Jr, Lubhan DB, O'Donnell TF Jr, Korach KS, Mendelsohn ME. Eserogen inhbits the vascular injury response in estrogen receptor O-deficient mice. Nar Med. 1997; 3: 545-548.

11. Karas RH, Patterson BL, Mendelsohn ME. Human vascular smooth musde cells contain functional etrogen receptor. Circulation. 1994; 89: 1943-1950.

12. Kim-Schulze S, McGowan KA, Hubchak SC, Cid MC, Martin MB, Kleinman HK, Greene GL. Schnaper HW. Expression of an estrogen recepror by human coronary artery and umbilical vein endothelial cells. Circulation. 1996; 94: 1402-1407.

13. Lindner V, Kim SK, Karas RH, Kuiper GGJM, Gustafsson J-A, Mendelsohn ME Increased expression of estrogen recepror B m RNA in male blood vessels following vasular injury. Circ Res. 1998; 83: 224-229.

14. Venkov $\mathrm{CD}$, Rankin $\mathrm{AB}$, Vaughan DE. Idenrification of authentic estrogen receptor in cultured endothelial cells: a potential mechanism for steroid hormone regulation of endothelial funcrion. Circulawion. 1996; 94: 727-733.

15. Losordo DW, Kearney M, Kim EA, Jekanowski J, lisner JM. Variable expression of dhe estrogen receptor in normal and atherosclerotic cononary arteries of premenopausal women. Cirulation. 1994; 89: 1501-1510.

16. Register TC, Adams MR. Coronary arrery and cultured aortic smooth muscle cells express mRNA for both the classical estrogen receptor and the newly described estrogen recepror B. I Steroid Biachem. Mol Biol. 1998; 64: 187-191.

17. Sullivan TR Jr, Karas RH, Aronovitz M, Faller GT, Ziar JP, Smith JJ, O'Donnell TF Jr, Mendelsoln ME. Estrogen inhibits the response-ro-injury in a mouse carotid artery model. / Clin Innest. 1995; 96:2482-2488.

18. Karas RH, Hodgin JB, Kwoun M, Krege JH, Aronovitz M. Mackey W, Gustafsson JA, Korach KS, Smithies O, Mendelsohn ME. Estrogen inhibits the vascular injury response in estrogen receptor B-deficien female mice. Proc Not Acad Sct USA. 1999; 96: 15133-15136.

19. Lubahn DB, Moyer IS, Golding TS, Couse JF, Korach KS, Smithies O. Alteration of reproductive function bur not prenatal sexual development after insertional distuption of the mouse estrogen receptor gene. Proc Nat Aad Sid USA. 1993; 90: 11162-11166.

20. Krege JH. Hodgin JB, Couse JF, Enmark E, Warner M, Mahler JF, Sar M, Korach KS, Gustafsson 1-A. Smithies $O$. Gencration and reptoducrive phenocype of mice lacking estrogen recepror $B$. Prot Nat Acad Sci USA. 1998; 95: 15677-15682.

21. Couse JF. Hewitt SC, Bunch DO, Sar M, Walker VR, Davis BJ, Korach KS. Postnatal sex reversal of the ovaries in mice lacking eatrogen receptors and B. Science. 1999; 286: 2328-2331.

22. Ogawa S, Chester AE, Hewirt SC, Walker VR, Gustafsson JA, Smithies O. Karach KS, Pfaff DW, From the Cover: Abolition of malle sexual behaviors in mice lacking estrogen receptors $\alpha$ and $B$ (OAERKO). Prof Nat Acad Sci USA. 2000; 97: 14737-14741.

23. Couse JF, Curris SW, Washburn TF, Lindzey J, Golding TS, Lubahn DB, Smichies O, Korach KS. Analysis of transcription and estrogen insensitiviry in the female mouse after targeted distuprion of the estrogen receptor gene. Mol Endacrinol. 1995; 9: 1441-1454. 
24. Widal O, Lindberg MK, Hollberg K, Baylink DJ, Andersson G, Lubhan DB, Mohan S, Gusrafsson $J A_{3}$ Ohlsson C. Estrogen receptor specificity in the regulation of skelecal growth and maruration in male mice. Proc Notl Acad Soi USA. 1997; 10:5474-5479.

25. Windah SH, Vidal O, Andersson G, Gustafson JA, Ohisson C. Increased corrical bone mineral content but unchanged rabecular bone mineral densiry in female ERR(w) mice. / Clin Inwest. 1999; 104: 895-901.

26. Lindner V. Fingerle J, Reidy MA. Mouse model of arterial injury. Circ Res. 1993; 73: 792-796.

27. Walter $P$, Green $S$, Green $G$, Krust A, Gornert JM, Jeltsch JM, Staub A, Jensen E, Scrace G, Waterfield $\mathrm{M}$, Chambon $\mathbb{P}$. Cloning of the human estrogen receptor CDNA. Proc Not Awa Soi USA. 1985; 82: 7889-7893.

28. Kuiper GG, Enmark E, Pelto-Huikko M, Nilsson S, Gustafsson JA. Cloning of a novel estrogen receptor expressed in rat prostate and owary. Proc Nat Acad Sai USA. 1996; '93: 5925-5930.

29. Mosselman S, Polman $\mathbb{I}$, Dijkema $\mathbb{R}$. ERR. Identification and characterization of a novel human estrogen receptor. FEBS Lett. 1996; 392: 49-53.

30. Tremblay GB, Tremblay A, Copetand NG, Gilberr DJ, Jenkins NA, Labrie F, Giguère V. Cloning, chromosomal localization, and functional analysis of the murine estrogen receptor B. Mod Endocrinol. 1997; 11:353-365.

31. Barkhem T, Carlsson B, Nilsson Y, Enmark E, Gustafsson J, Nilsson S. Differential response of estrogen receptor $\alpha$ and estrogen receptor $B$ to partial estrogen agonists/antagonists. Mol Pharracol. 1998: $54: 105-112$

32. Gïguère V, Tremblay A, Tremblay GB. Estrogen receptor R: re-evaluation of estrogen and antiestrogen signaling. Sterojds. 1998; 63: 335-339.

33. Paech K, Webb P, Kuiper GGJM, Nilsson S, Gustafsson JA, Kushner PJ, Scanlan TS. Differential ligand activation of estrogen receptors ER $\alpha$ and ERB at AP1 sites. Sciente. 1997; 277: 1508-1510.

34. Montano MM, Ekena K, Delage-Mourroux R, Chang WR, Marrini P, Kaczenellenbogen BS. An estrogen receptor-selective coregulator that potentiates the effectiveness of antiestrogens and represses the activity of estrogens. Froc Nar Aad Si USA. 1999; 96:6947-6952.

35. Poelzl G, Kasail Y, Mochizuki N, Shaul PW, Brown M, Mendelsohn ME. Specinc association of estrogen receptor with a cell cycle spindle assembly checkpoint protein. MAD2. Proc Nat Awat $\mathrm{So}$ USA. 2000; 97: 2836-2839.

36. Sun J, Meyers MJ, Fink BE, Rajendran R, Karzenellenbogen JA, Kaczend lenbogen BS. Novel ligands that function as selective estrogets or antiestrogens for estrogen recepor a or estrogen recep. ror B. Endocrinology 1999; 140:800-804.

37. Chang C, Norris JD, Grohn H, Paige LA, Hamilton PT, Kenan DJ, Fowlkes D, McDonnell DP. Dissection of the LXXLL nuclear receptor-coacrivaror interacrion motif using combinatorial pepride libraries: discovery of peptide antagonists of estrogen receptors a and B. Mol Cell Biol. 1999; 19: $8226-8239$.

38. Norris JD, Paige LA, Christensen DJ, Chang CY, Huani MR, Fan D, Hamitron PT, Fowlkes DM. McDonnell DP. Peptide antagonists of the human estrogen receptor. Schene. 1999; 285: $744-746$. 
39. Hodgin JB, Krege JH, Reddick RL, Korach KS, Smithies O, Madeda N. Estrogen recepror $\alpha$ is a major mediator of 17 -estradiol's atheroprotecrive effects on lesion size in Apoe-1- mice. J Clin Invest. $2001 ; 107: 333-340$.

40. Couse JF, Korach KS. Estrogen receptor null mice: what have we learned and where will they lead us? Endocr Rev. $1999 ; 20: 358-417$.

41. Mangelsdorf DJ, Thummel C, Beato M, Herrlich P, Schutz G, Unesono K, Blumberg B, Kastner P, Mark M, Chambon P. Ewans RM. The nudlear receptor superfamily: the second decade. Cell 1995; 83: 835-839.

42. Dupont $S$, Krust A, Gansmuller $A$, Dierich $A$, Chambon $P$, Mark $M$. Effecr of single and compound knockours of estrogen receprors $\alpha(E R \alpha)$ and $B$ (ERB) on mouse reproductive phenorypes. Developwitut. 2000; $127: 4277-4291$. 
CHAPTER 4

\section{Nitric oxide synthase(s) mediate the vascular protective effect of estrogen in the mouse carotid artery injury model}

Henny M. Schulten, Richard H. Karas, Mark Aronovitz, Gary Pare, Flore Celestin, Ebo D. de Muinck, and Micheal E. Mendelsohn. 


\begin{abstract}
Estrogen increases bioawailabilicy of the endogenous vasodilator and wascular protective molecule, nitric oxide. We have previously shown that $17 \beta$-estradiol $\left(E_{2}\right)$ inhibits the response to vascular injury in the mouse carotid injury model. We examined the effect of L-NAME, a non-selective nitric oxide synchase (NOS) inhibitor, on $E_{2}$ mediated prorection against vascular injury. Forry-one ovariecromized $\mathrm{C} 57 \mathrm{BL} / 6 \mathrm{~J}$ femalle mice were randomized to receive vehicle alone, $E_{2}, L-N A M E$, or $\mathbb{L}-N A M E+E_{2}$. Mice were subjected to unilateral carotid arterial injury and the response to injury was assessed by independent measures of carotid medial area (MA) and vascular smooth muscle cell (VSMC) proliferation rwo weeks later. As compared to wehicle-treated mice $(102.2 \pm 0.3 \mathrm{mmHg}), \mathrm{L}$ NAME rrearment significantly increased mean systolic blood

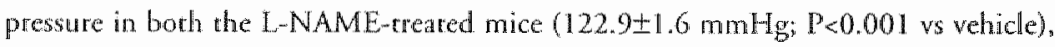
and in the L $L A M E+E_{2}$-treated mice $(110.8 \pm 2.2 \mathrm{mmHg}, P<0.001$ ws wehicle). Estrogen decreased blood pressure in both the $\mathrm{E}_{2}$-treated mice as compared ro control animals (95.9 $\pm 1.3 \mathrm{mmHg} ;$ P 0.01 vs vehicle), and the L-NAME+E 2 -treated mice, as compared to mice treated with L-NAME alone $(110.8 \pm 2.2 \mathrm{mmHg} ; \mathbb{P}<0.001$ ws L-NAME). Injury induced a significant increase in MA in wehicle-rreared mice that was reversed in $E_{2}$-rreared mice ( $P<0.05$ ws vehicle-rreared). In $\mathrm{L}$-NAME-rreated mice, injury caused an increase in MA similar to that seen in vehicle-treated mice ( $P=N S$ vs vehicle-reated. However, $\mathrm{E}_{2}$ treament had no protecrive effect in mice receiving L-NAME. In vehicle-treated animals, calrotid injury induced an increase in BrdU-labeled VSMCS $(P I=0.37 \pm 0.04 ; \mathbb{P}<0.001)$ that $E_{2}$ inhibited $(P I=0.21 \pm 0.03$; P<0.01 vs vehicle-treared). L-NAME alone had no significan effect on the degree of injury-induced VSMC proliferation. In L-NAME $+E_{2}$ animals, estrogen no longer suppressed the vascular injury response. Therefore, pharmacological inhibition of nitric oxide synthases abolishes rhe protective effects of $\mathbb{E}_{2}$ on medial area and VSMC proliferation in the mouse carotid injury model. These findings support that the wascular protective effects of $\mathrm{E}_{2}$ in wivo are due to estrogen receprom-mediared activation of one or more NOS enzymes.
\end{abstract}




\section{INTRODUCTION}

Recent randomized clinical trials of conjugated equine estrogens and medroxyprogesterone acetate in older women, with or without known atherosclerosis, have shown no benefit and there were some trends toward harm. ${ }^{1-3}$ In conrrast, several large-scale observational studies have shown reduced cardiovascular risk associared with use of hormone replacement therapy. 4,5 The effects of various hormone replacement regimens on both non-vascular and cardiovascular tissue are complex, underscoring the importance of understanding the molecular mechanisms by which hormones regulate vascular biology.

Animal models provide one useful approach to evaluating mechanisms by which estrogen can exert vascular protective effects. ${ }^{6}$ The mouse model of carotid arterial injury has proven useful in understanding the role of estrogen receptors (ER) $\alpha$ and $\beta$ in mediating the protective cardiovascular effects of estrogen. Using this model, we have shown previously that administration of physiologic levels of $17 \beta$-estradiol $\left(\mathrm{E}_{2}\right)$ completely inhibits the vascular injury response in ovariectomized female WT" mice, an effect that is mediated by $E R \alpha$ in this model..$^{7-10}$ Estrogen also accelerates re-endothelization of injured murine carotid arteries through ER $\alpha .{ }^{11}$ However, the molecular pathways downstream of estrogen receptor $\alpha$ responsible for mediating the protecrive effects of estradiol/ER $\alpha$ on vascular injury remain unknown. ${ }^{6,12}$

Nitric oxide (NO), which has multiple important vascular protective effects, is one likely candidate to explain the beneficial effects of estrogen. Estrogen has previously been shown to augment NO bioavailability, acutely and following prolonged exposure, both in vitro and in vivo, ${ }^{6,11,13-16}$ Nitric oxide is produced by three isoforms of nitric oxide synthase (NOS). All three NOS isoforms are detectable in the vasculature, and data supporting a protective role of each have been reported. ${ }^{17-22}$ To test the hyporthesis that NO produced by one or more of these NOS isoforms mediates estrogenic protection against vascular injury, we examined the effects of a non-selecrive pharmacologic NOS inhibitor on estrogen protection against the vascular injury response in the mouse carotid injury model.

\section{MATERIALS AND METHODS}

\section{ANIMALS}

Ten-twelve week old, female C57BL/6J mice (45) were purchased from Jackson Laboratories (Bar Harbor, ME). Four animals were housed per cage in a temperature-controlled facility on a 12 hour light-dark cycle and were fed a normal dict ad libirum, as previously described.7,8 Institutional guidelines of the Institutional Animal Care and Use committee, and of the Department of Laborarory Animals of 
Tufts University School of Medicine and the Tufts New England Medical Center were followed.

\section{MOUSE MODEL OF CAROTID ARTERIAL INJURY}

The mouse carotid injury model used in this study has been described in detail previously. ${ }^{7-9,23,24}$ Forty-five adult female mice were ovariectomized and allowed to recover for $7-10$ days. On Day-7, mice were randomly assigned to receive vehicle alone (vehicle), $\mathrm{E}_{2}$-releasing pellets $\left(\mathrm{E}_{2} ; 0.1 \mathrm{mg}, 21\right.$-day release pellers, Innovarive Research of America), $N^{G}$-nitro-arginine-methyl ester (L-NAME; Sigma Chemical Co., St. Louis, Missouri, USA), a non selective inhibitor of nitric oxide synthase inhibitor (1.5 gram $/ \mathrm{L}$ in drinking water; refreshed every two days) or both L-NAME and $E_{2}\left(L-N A M E+E_{2}\right)$. On day 0 , the mice underwent unilateral common carotid artery injury by intraluminal passage of a fine wire, denuding the vascular endothelium and inducing an characteristic increase in medial area and VSMC proliferation. On the same day as the carotid injury was performed, the mice were implanted subcutaneously with an osmotic minipump (Alzet Inc., Palo Alto, California, USA) that continuously releases bromo-deoxy-uridine (BrdU: $25 \mathrm{mg} / \mathrm{kg} / \mathrm{d}$ ) throughour the experimental period.

\section{BLOOD PRESSURE MEASUREMENTS}

Blood pressure was measured in conscious animals on day-7, 0, 3, 10 and 14 . Systolic blood pressure was measured by the tail-cuff method using a BP-2000 Specimen Platform (Visitech System, Cory, NC). Before the start of the experiment all animals were acclimated to the procedures involved with measuring blood pressure. Blood pressures were measured during 4 intervals, each lasting 5 minutes, during which 10 blood pressure measurements were taken. The reported blood pressures represent the means of all of these measurements. An investigator blinded to the animals treatment group assignment performed all measurements.

\section{MORPHOMETRY AND IMMUNOHISTOCHEMISTRY}

Two weeks following vascular injury, the mice were anesthetized again with $2.5 \%$ isoflurane and both carorid arteries were harvested after perfusion fixation with $\rrbracket 0 \%$ formalin at $150 \mathrm{mmHg}$ for 4 minutes. Ureri were also removed and wet weights were obtained. The response to wascular injury was assessed using two measures: MA (medial area) and VSMC proliferarion. Parallel sections from all carorid arteries were stained as described previously for hematoxyline/eosin and for elastin, and MA measurements were made using a computerized morphometric analysis system on the elastin-stained sections (Image Pro Plus software), ${ }^{8,9,23}$ Immunostaining also was performed on parallel carorid artery sections using anti-BrdU antibodies (Sigma 
Chemical Co., St. Louis, Missouri, USA), and VSMCs that were labeled with BrdU were quantified as described. $8,9,23$ Adventirial cells were not included in any of these analyses. Proliferation Index (PI) was calculated as the ratio of BrdU-positive VSMC to total VSMC per section. Two observers blinded to treatment group performed all analyses.

\section{STATISTICAL ANALYSES}

All values are expressed as mean $\pm S E M$. For statistical analyses of blood pressure, time and treatment group were considered independent variables and a 2-factor ANOVA was performed. Post-hoc pairwise comparisons were made with the Student-Newman-Keuls test. All other statistical analyses were tested by 1-factor ANOVA, with post-hoc pairwise comparisons made with the Student-Newman-Keuls test. Differences were considered statistically significant at $P<0.05$.

\section{RESULTS}

Four animals died during the surgery and three animals $\left(2 E_{2}\right.$-treated and 1 L-NAME-treated) had completely thrombosed arteries at the time of sacrifice. These animals were excluded from further analysis. At the time of injury there were no differences in body weight berween the experimental groups (placebo, 23.4 $\pm 0.4 \mathrm{~g}$; $E_{2}, 23.8 \pm 0.3 \mathrm{~g} ; \mathrm{L}-\mathrm{NAME}, 22.9 \pm 0.4 \mathrm{~g} ; \mathrm{L}-\mathrm{NAME}+\mathrm{E}_{2}, 23.0 \pm 0.4 \mathrm{~g}, \mathrm{P}=\mathrm{NS}$ ). Estrogen treatment resulted in the expected increase in uterine weight $t^{9,10}$ from $9.5 \pm 0.8 \times 10^{-3}$ and $9.9 \pm 1.0 \times 10^{-3} \mathrm{~g}$ (placebo and L-NAME, respectively) to $74.1 \pm 5.6 \times 10^{-3}$ and $75.1 \pm 5.7 \times 10^{-3} \mathrm{~g}$ (respectively, $\mathrm{E}_{2}$ and $\mathrm{L}-\mathrm{NAME}+\mathrm{E}_{2}$ groups, $\mathrm{P}<0.001$ ). These data confirm the appropriate delivery of and a physiologic response to $\mathrm{E}_{2}$ in the $E_{2}$-treated animals.

The mean systolic blood pressure in the vehicle-rreated mice was $102.2 \pm 0.3$ $\mathrm{mmHg}$. L-NAME treatment significantly increased mean systolic blood pressure in borh the L-NAME mice $(122.9 \pm 1.6 \mathrm{mmHg}$; Figure $2 ; \mathrm{P}<0.001$ vs Vehicle), and in the $\mathrm{L}-\mathrm{NAME}+\mathrm{E}_{2}$ mice ( $110.8 \pm 2.2 \mathrm{mmHg}, \mathrm{P}<0.001$ ws Vehicle). Estrogen significantly decreased mean blood pressure in both the $\mathrm{E}_{2}$ mice $(95.9 \pm 1.3 \mathrm{mmHg}$; Figure 2; $P=0.008$ ws vehicle), and the $L-N A M E+E_{2}$ mice, as compared to mice treated with

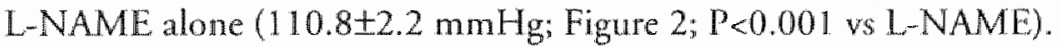

\section{EFFECTS OF $E_{2}$ ON MEDIAL AREA}

The MA of the uninjured (uninj) vessels was $13.1 \pm 0.4 \times 10^{-3} \mathrm{~mm}^{2}$. Injury induced an increase in $\mathrm{MA}$ to $18.4 \pm 0.6 \times 10^{-3} \mathrm{~mm}^{2}$ in the vehicle-treated mice (Figures 3 and 4, $\mathrm{P}<0.001)$. As in previous studies, ${ }^{7-10,23} \mathrm{E}_{2}$ replacement inhibited the injury-induced increase in medial area to levels comparable to the uninjured vessels 

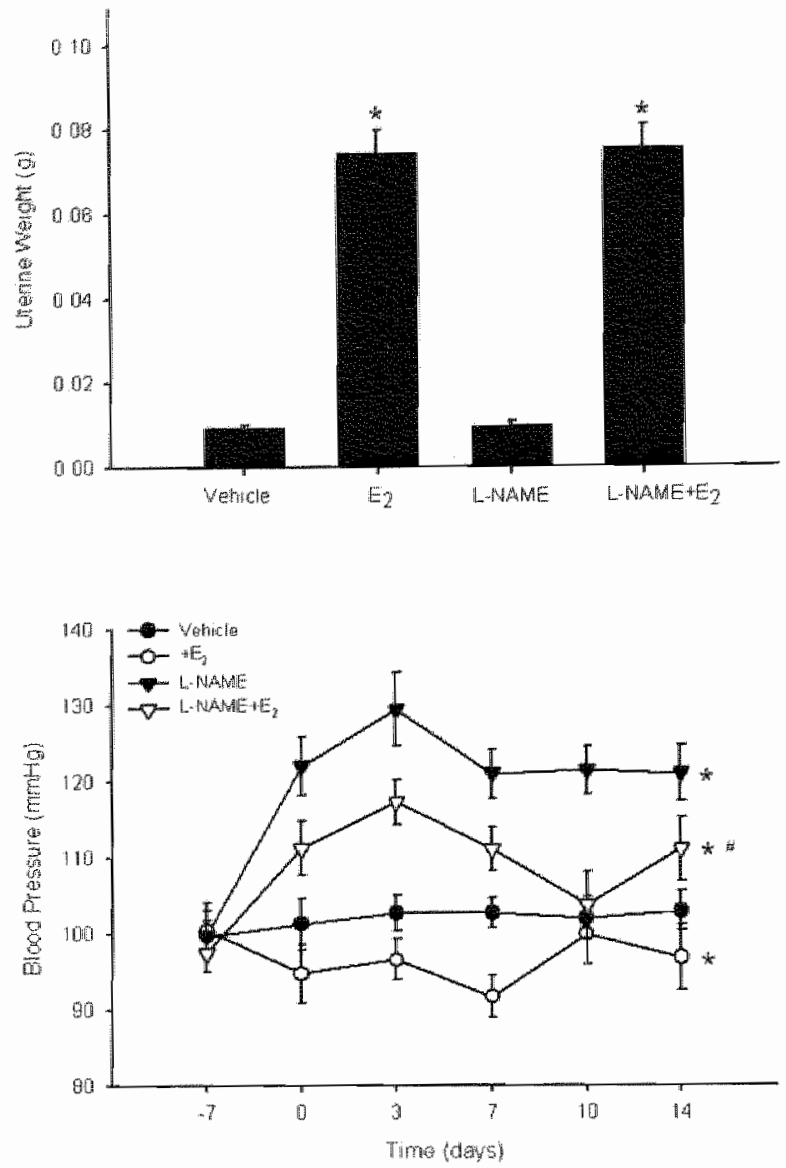

Fugure 1. Uterine weights of C57BL/6] mice reared with wehlde, $E_{2}$, L-MAME or L-NAME $+E_{2}$. The increases in uterine weights in the estrom gen-treated groups is highly significant compared with the non-estrogen-treated groups. Bars represent the meant $S E M$ for each group. $\mathrm{P}<0.001$ vs vehicle or L-NAME.
Figure 2. Mean systolic blood pressure measurements in study groups over time. Bars represent the mean \pm SEM for each group. $* 00.001$ ws vehicle-treated animals; $\mathrm{P}<0.001$ vs L-NAME.

$\left(15.1 \pm 1.1 \times 10^{-3} \mathrm{~mm}^{2}, \mathrm{P}<0.05\right.$ ws vehicle; $\mathrm{P}=\mathrm{NS}$ vs uninj). L-NAME alone did not affect the magnitude of injury-induced increase in MA $\left(20.8 \pm 1.6 \times 10^{-3} \mathrm{~mm}^{2} ; \mathrm{P}=\mathrm{NS}\right.$ vs vehicle-treated). However, $E_{2}$, was not able to inhibit the injury-induced increase in MA in the L-NAME $+E_{2}$ animals (Figures 3), 20.0 $\pm 1.5 \times 10^{-3} \mathrm{~mm}^{2} ; \mathrm{P}=\mathrm{NS}$ vs L-NAME alone; $P<0.01$ vs vehicle-treated).

\section{EFFECTS OF E ON VSMC PROLIFERATION}

As in all previous studies with this mode $7^{7-10} \mathrm{BrdU}$-labeled cells were detected only rarely in the uninjured arteries (mean $<1$ cell per section). Carotid injury induced a pronounced increase in BrdU-labeled VSMCs in the vehicle-treated animals (proliferation index $(\mathrm{PI})=0.37 \pm 0.04 ; \mathrm{P}<0.001)$. $\mathrm{E}_{2}$ significantly inhibited this increase $(P \mathrm{I}=0.21 \pm 0.03 ; \mathrm{P}<0.01$ vs vehicle-treated, Figure 5), as expecred.9,10 L-NAME 

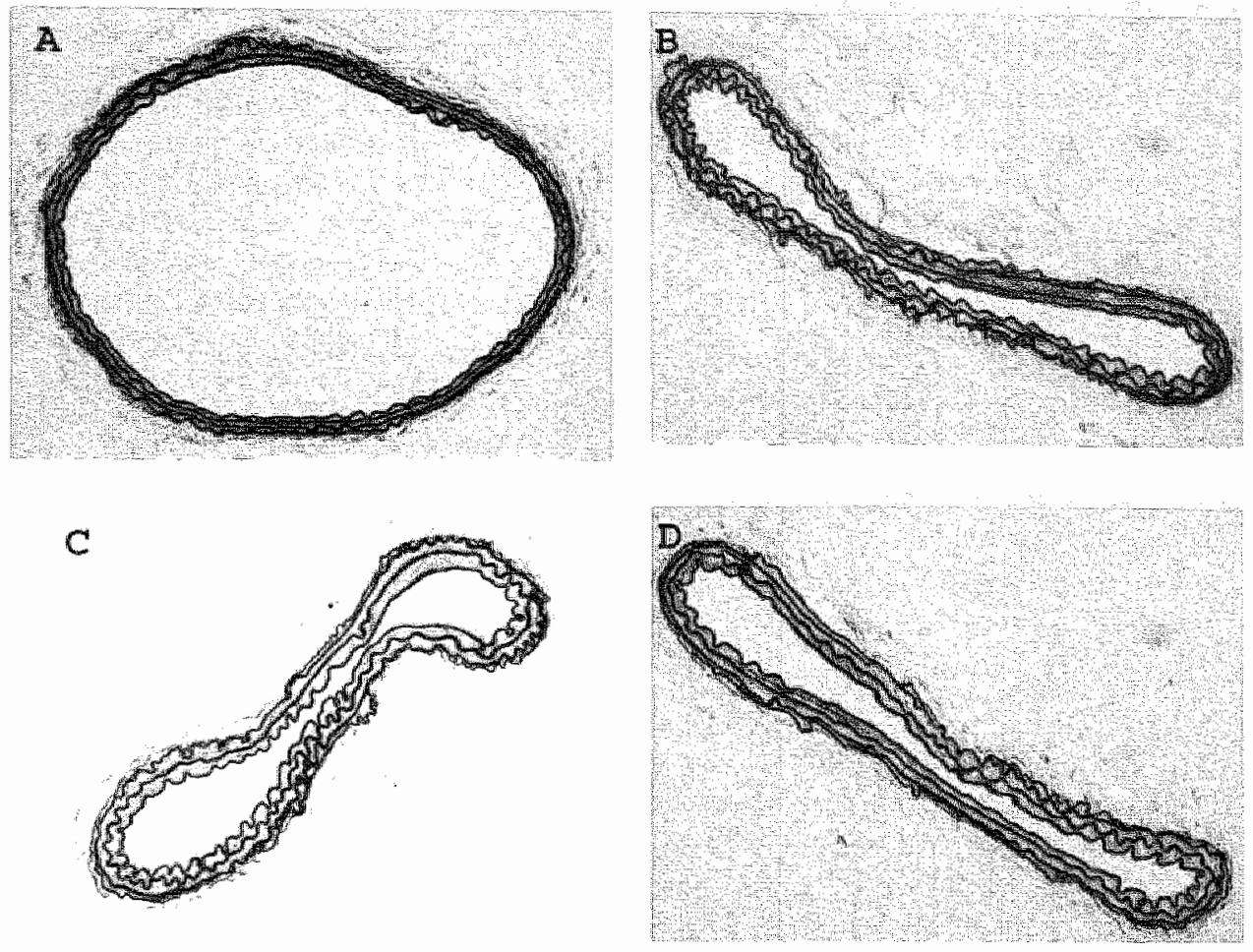

Figure 3. Representative sections stained for measurement of medial area. (A-E) Representative segments from elastin-stained carotid artery sections are shown. All area measurements were made on complete sections. (A) uninjured, (B) injured, vehicle-treated, (C) injured, E-treated, (D) injured, L-NAME-treated, (E) injured, L-NAME $+E_{2}$-rreared.

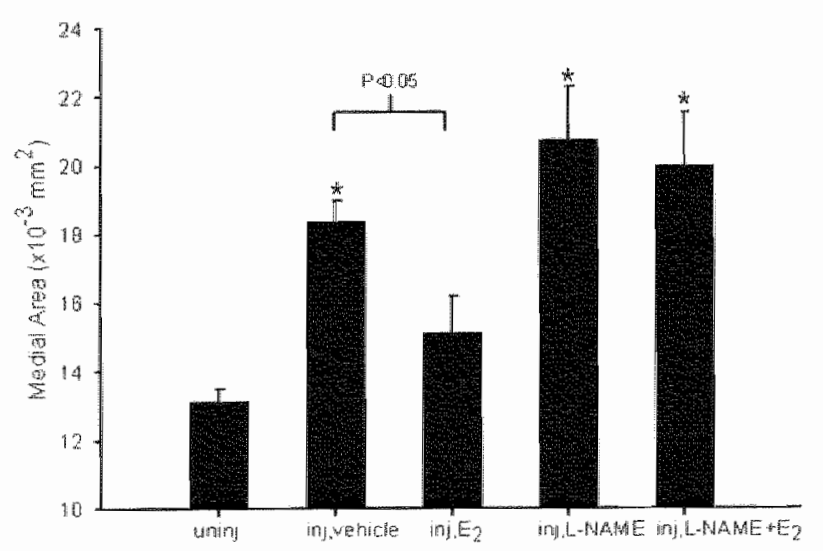

Figure 4. Medial area of uninjured (uninj) and injured (inj) ca. rond arteries from mice neceiving wehicle, $\mathbb{E}_{2}$, L-NAME or L.NAME $+E_{2}$. $E_{2}$ replacement significantly inhibiced the injury induced increase in medial area to levels comparable to the uninjured animals in the absence of L-NAME E $E_{2}$ replacement had no effect on the injury-induced increase in the presence of L-NAME. Bats represent the mean $15 E M$ for each group. $* P<0.001$ vs uninj. 


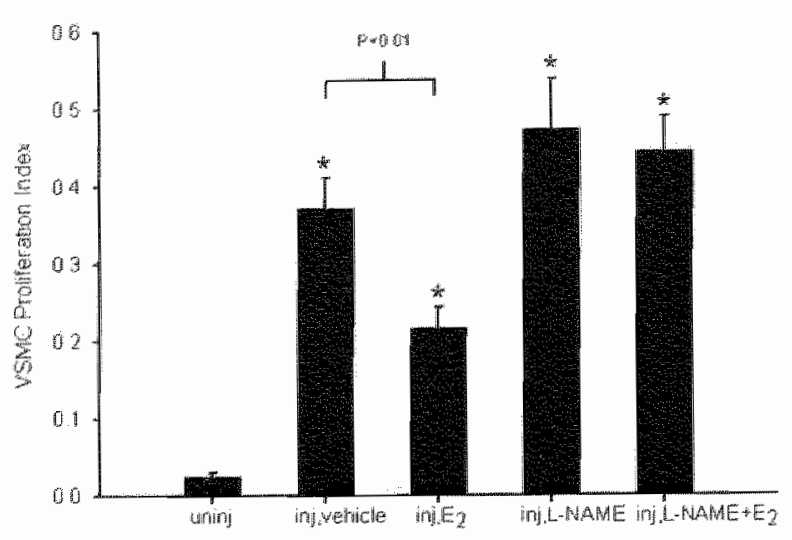

Figure 5. Vascular smooth muscle cell proliferation of uninjured (mninj) and injured (inj) carorid arreries from mice rreated with wehicle, $\mathrm{E}_{2}$ LNAME, or $\mathrm{L}-\mathrm{NAME}+\mathrm{E}_{2}$. E $\mathrm{E}_{2}$ inhibired VSMC proliferation significantly, in the absence of L-NAME. E $E_{2}$ had no effect on injury-induced increases in VSMC proliferation in the presence of L-NAME. Bars represent the mean $\$$ SEM for each group. * $P<0.00$ 1 vs uninj.

alone had no significant effect on VSMC proliferation following injury $(0.47 \pm 0.06$; $\mathrm{P}=\mathrm{NS}$ vs velicle-treated). As with the medial area, estrogen's ability to protect against vascular injury was completely abolished in the $\mathrm{L}-\mathrm{NAME}+\mathrm{E}_{2}$ animals (PI $=0.44 \pm 0.04 ; P=N S$ ws L-NAME alone; $P<0.01$ vs vehicle-treated, Figure 5).

\section{DISCUSSION}

This study used the mouse carotid injury model to examine the mechanism by which estrogen inhibits medial thickening and VSMC proliferation following vascular endothelial denudation injury in ovariectomized fernale mice. Our data support that the vascular prorective effects of estrogen in this model are mediated by one or more of the three nitric oxide synthase isoforms, eNOS, iNOS and/or nNOS, through which specific isoform(s) are responsible remains unclear.

It is important to consider the potential impact on our findings of differences in blood pressures between the treatment groups. As expected, both groups of mice that received L-NAME had significant elevations in mean systrolic blood pressures. Though the presence of hypertension could offset estrogen's protective effect, hypertension-mediated inhibition of the response to injury in the L-NAME $+E_{2}$ mice seems unilikely for at least two reasons. First, despite the significant increase in blood pressure in the L-NAME mice, there was no significant difference in the magnitude of the response to vascular injury between the L-NAME and the vehicle-treated mice. Second, the addition of $\mathrm{E}_{2}$ treatment in the L-NAME $+\mathrm{E}_{2}$ group significantly decreased blood pressure compared to the L-NAME group, but this had no effect on the response to vascular injury. Thus, it seems unlikely that L-NAME's effects on blood pressure contributed in any important way to the current findings. It is of interest that $E_{2}$ treatment was able to lower blood pressure even in the L-NAME-treated animals. Though it is possible that this was due to an effect of $\mathrm{E}_{2}$ 
on NOS activiry despite the presence of high-dose L-NAME, it seems more likely that this is due to NOS-independent effects of $\mathrm{E}_{2}$ on blood pressure regulation. One potential parhway by which this could occur is by $\mathrm{E}_{2}$-mediated inhibition of central sympathetic nerve activity, which can be induced by L-NAME. ${ }^{25.26} \mathrm{E}_{2}$ has previously been shown to inhibit sympathetic outflow in ovariectomized rats, ${ }^{27}$ and $\mathrm{E}_{2}$-receptors have been identified in brain centers involved in cardiovascular regulation. 28,29

Estrogen causes an estrogen receptor-dependent but non-genomic increase in eNOS activity. ${ }^{13,15,30-34}$ Estrogen also induces the expression of eNOS, 35 iNOS, ${ }^{36,37}$ and nNOS ${ }^{35,38}$ genes in vascular cells. Furthermore, estradiol increases iNOS expression in macrophages ${ }^{39}$ and neuronal nitric oxide protein expression in neutrophils. ${ }^{40}$ There are therefore multiple explanations for the effects of L-NAME noted in the present study. The mouse carotid injury model is based on quantification of the vascular response to an endochelial denudation injury in normolipidemic animals. Very few, if any, white blood cells are noted in the injured vessels in this model (RKH and MEM, unpublished observations). In addition, in mice there is no published evidence at present for the expression of $\mathrm{nNOS}$ in the vessel wall and repeated attemps made to detect nNOS in the vasculature in one of our prior studies were unsuccessful. ${ }^{22}$ Therefore, the most plausible mechanism for estrogen-mediated protecrion against vascular injury response in this model is that estrogen-estrogen receptor alpha ${ }^{10}$ induced $\mathrm{NOS}$ and/or iNOS in the vasculature of wild-type mice. Because the non-specific NOS inhibitor L-NAME was used in this study, it will be necessary to address whether $\mathrm{NOS}$ and/or iNOS are responsible for mediating protection in subsequent studies with NOS-specific knockout mice.

In conclusion, the current findings demonstrate that estrogen-mediated protection against vascular injury is blocked by co-administration of the non-specific NOS inhibitor L-NAME. This observation supports that $\mathrm{E}_{2}$-mediated protection from vascular injury is mediated by one or more NOS isoforms. These findings have important implications of the in vivo molecular parhways that mediate the vascular protective effects of estrogen.

\section{REFERENCES}

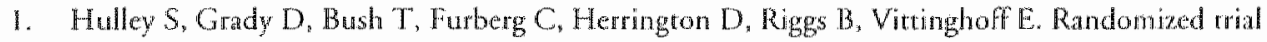
of estrogen plus progesrin for secondary prevention of coronary heart disease in posmenopausal women. Heart and Estrogen/progestin Replacement Sudy (HERS) Rescarch Group. Jama. $1998 ; 280: 605-13$.

2. Herrington DM, Reboussin DM, Brosnihan KB, Sharp PC, Shumaker SA, Snyder TE, Furberg CD, Kowalchuk GI, Stuckey TD, Rogers WJ, Givens DH, Waters D. Effects of estrogen replacement on the progression of coronary-artery atherosderosis. N Engl/ Med. 2000;343:522-9. 
3. Rossuw JE, Anderson GL, Prentice RL, LaCroix AZ, Koopertberg C, Srefanick ML, Jackson RD, Beresford SA, Howard BV, Jolmason KC, Kotwen JM, Ockene J. Risks and benefins of estrogen plus progestin in healtyy postmenopausal women: principal results From the Women's Health Initiative randomized controlled trial. Jama. 2002;288:321-33.

4. Grimes DA, Lobo RA. Perspectives on the Wonnen's Health Initiative trial of hormone replacement therapy. Obstet Gynecol. 2002:100:1344-53.

5. Herrington DM, Klein KP. Randomized dinical orials of hormone replacement therapy for treatment or prevention of cardiovascular disease: a review of the findings. Atberosderosis. $2003 ; 166: 203-12$.

6. Mendelsohn ME; Karas RH. The prorective effects of estrogen on the cardiovascular system. $N$ Engl/ Med. 1999;340:1801-11.

7. Sullivan TRR, Jr, Karas RH, Aronovitz M, Faller GT, Ziar JP, Smith Jj, O. Donnell TF J, Mendelsohn ME. Estrogen in hibits the response-to-injury in a mouse caronid artery model. $J$ Clin Invest, 1995;96:2482-8.

8. Vafrati MD, Karas RH, Aronovitz M, Kim $\$$, Sullivan TR, Jr., Lubahn DB, O. Donnell TF J, Korach KS, Mendelsohn ME. Estrogen inhibirs che vascular injury response in estrogen receptor alphateficient mice. Nat Med. 1997;3:545-8.

9. Karas RH, Hodgin JB, Kwoun M, Krege JH, Aronovirz M, Mackey W, Gustafsson JA, Korach KS, Smithies O, Mendelsohn ME. Estrogen inhibits the vascular injury response in estrogen receptor beta-deficient female mice. Proc Natl Acad Sci US A. 1999;96:15133-6.

10. Pare G, Krust A, Karas RH, Dupone S, Aronoviz M, Chambon P, Mendelsohn ME. Escrogen Receproralpha Mediates the Protective Effects of Estrogen Against Vascullar Injury. Circ Res. 2002;90:1087-92.

11. Brouchet L, Krust A, Dupont S, Chambon P, Bayard F, Arnal JF. Estradiol accelerates reendothelializarion in mouse carorid artery through estrogen receptor-alpha but not estrogen receptor-beta. Cinculation. 2001;103:423-8.

12. Luscher TF, Barron M, Wight E, Espinosa E, Yang Z [Action of narural estrogens on the vessel wall: molecular mechanisms and dinical implications]. Schutez Med Wochensotro. $1996: 126: 1748-55$.

13. Mendelsoln ME. Nongenomic, ER-mediated activation of endothelial nitric oxide synthase: how does it work? What does it mean? Cir Res. 2000;87,956-60.

14. Pendaries C, Dablade B. Rochaix P" Krust A, Chambon P, Korad KS, Bayard P, Amal IF. "The AF-1 actiwation-function of ERalpha may be dispensable ro mediate the effect of estradiol on encorhelial NO production in mice. Proc Narl Aad Sci US A. 2002;99:2205-10.

15. Chambliss KI, Shaul PW. Estrogen modularion of endorhclial nitric oxide synthase. Endocr Rev. 2002:23:665-86.

16. Haynes MP, Li L, Sinha D, Russell KS, Hisanoto K, Baron R. Collinge M, Sessa WC, Bender JR. Sre kinase mediates phosphatidylinositol 3-kinase/Akt-dependent rapid endothelial nitric-oxide synthase activation by estrogen. J Biol Chem. 2003;278:2118-23.

17. Michel 'T, Feron O. Nitric oxide synthases: which, where, how and why? J Clin Inwest. 1997;100:2146-52. 
18. Yan $Z$ Q, Yokota $T$, Zhang W, Hanson GK. Expression of inducible nirric oxide synthase inhibits platelet adhesion and restores blood flow in the injured artery. Cin Re. 1996;79:38-44.

19. Yan $Z$, Hansson GK. Overexpression of inducible nirric oxide synthase by neointimal smooth muscle cells. Circ Res. 1998:82:21-9.

20. Muller B, Kleschyov AL, Gyorgy K, Stoclet JC. Inducible NO synthase activity in blood vessels and hearr: new insight into cell origin and consequences. Pbywo/ Res. 2000:49:19-26.

21. Zhu $Y$, Bian Z, Lu P, Karas RH, Bao L, Cox D, Hodgin J, Shanl PW, Thoren P, Smithes O. Gustafsson JA, Mendelsohn ME. Abnormal vascular function and hypertension in mice deficient in estrogen recepror beta. Science. 2002;295:505-8.

22. Morishita $T$, Tsursui M, Shimokawa H, Horiuchi M, Tanmono A, Suda O, Tasalki H, Huang PL, Sasaguri Y, Yanaghara N, Nakashima Y. Vasculoprotective Roles of Neuronal Nitric Oxide Symthase. Faseb J. 2002;18:18.

23. Karas RH, Schulten H, Pare G, Aronowiz MJ, Ohlsson C, Gustafsson JA, Mendelsohn ME. EFfects of estrogen on the vascular injury response in estrogen receptor alpha, beta (double) knockout mice. Cir Ress. 2001;89:534́-9.

24. Lulbahn DB, Moyer JS, Golding TS, Conse JF, Korach KS, Smithies O. Alteration of reproductive function but not prenatal sexual development after insertional disruption of the mouse estrogen receptor gene. Proc Natl Acad Sci U S A. 1993;90:11162-6.

25. Togashi H, Sakuma I, Yoshioka M, Kobayashi T, Yasuda $\mathbb{H}_{4}$, Kitabatake A, Sairo H, Gross SS, Levi R. A central nervous system action of nitric oxide in blood pressure regulation. J Phampacol Exp Ther. 1992;262:343-7.

26. Sakuma I, Togashi H, Yoshioka M, Saita H, Yanagida M, "Tamura M, Kobayashi T, Yasuda H, Gross 5 , Lewi $\mathbb{R}$. NG-medyl-L-arginine, an inhibitor of L-arginine-derived nitric oxide synthesis, stimulates renal sympathetic nerve activity in vivo. A role for nitric oxide in the central regulation of sympathetic tone? Circ Res. 1992;70:607-11.

27. He XR, Wang W, Crofton IT, Share L. Effects of 17 beta-estradiol on the baroreflex control of sympathetic acrivity in conscious owartectomized rats. Am f Pbysol. 1999;277: R493-8.

28. Pellerier $\mathrm{G}, \mathrm{Lia}$ N, Follea N, Govindan MV. Mapping of estrogen recepror-producing cells in the rar brain by in situ hybridization. Neuroscr Lett. 1988;94:23-8.

29. Simonian $\mathrm{SX}$, Herbison AE. Differential expression of estrogen receptor and neuropeptide $Y$ by brainstem $A 1$ and $A 2$ noradrenaline neurons. Nettroscience. 1997:76-517-29.

30. Lantin-Hemoso RL, Rosenfeld CR, Yuhanna IS, German Z, Chen Z, Shatul PW. Estrogen acutely stimulates nitric oxide synthase activity in fetal pulmonary artery endothelium. Amy I Ptoysiol 1997:273:L119-26.

31. Caulin-Glaser T, Garcia-Cardena G, Sartel P, Sessa WC, Bender JR. 17 beta-estradiol regularion of human endothelial cell basal nitric oxide rellease, independent of cytosolic Ca2+ mobilization. Cre Res. 1997;81:885-92.

32. Chen Z, Yuhanna 1S, Galchewd-Gargova Z, Karas RH, Mendelsohn ME, Shaul PW. Estrogen receptor alpha mediates the nongenomic activation of endorhelial nitric oxide synthase by estrogen Ipublished erratum appears in I Clim Inwest 1999 May; 103(9):1363!. I Clin fonvest. $1999 ; 103: 401-6$. 
33. Haynes MP, Sinha D, Russell KS, Collinge M, Futron D, Morales-Ruiz M, Sessa WC, Bender JR. Membrane estrogen receptor engagement activates endothelial nitric oxide synthase via the P13-kinase-Ak pathway in human endothelial cells. Circ Res. 2000:87:677-82..

34. Simoncini T, Hafez-Moghadarn A, Brazl DP, Ley K, Chin WW, Liao IK Interaction of oestrogen receptor with the regulatory subunit of phosphatidylinositol-3-OH kinase. Natwre. $2000 ; 407: 538-41$

35. Weiner CP, Lizasoain I, Baylis $S A$, Knowles $R G$, Charles $I G$, Moncada S. Induction of calcium-dependent nitric oxide synthases by sex hormones. Proc Natl Acad Sid U $S$ A. 1994;91:5212-6.

36. Binko J, Majewski H. 17 beta-Estradiol reduces vasoconstricrion in endorhelium-denuded rat aortas through inducible NOS. Am J Physiol. 1998;274:17853-9.

37. Mershon JL, Baker RS, Clark KE. Estrogen increases iNOS expression in the ovine coronary artery. Ann J Physiol Heawt Circ Pbysiol. 2002;283:H1169-80.

38. Salhab WA, Shaul PW, COx BE, Rosenfeld CR. Regulation of types I and III NOS in ovine uterine arteries by daily and acute estrogen exposure. Am I Physiol Heart Circ Plyysiol. $2000 ; 278: 12134-42$.

39. You HJ, Kim JY, Jeong HG. 17 [beta]-Estradiol increases inducible nitric oxide synthase expression in macrophages. Brochemical and Biophysical Resarch Communications. 2003;303:11:29-1134.

40. Garcia-Duran M, de Frutos T, Diaz-Recasens J, Garcia-Galvez G, Jimenez A, Monton M, Farre J, Sanchez de Miguel L. Gonzalez-Fernandez F, Arriero MD, Rico L. Garcia R, Casado S, Lopez-Farre A. Estrogen stimulates neuronal nitric oxide synthase protein expression in human neurrophils. Cinc Res. 1999;85:1020-6. 


\section{Disruption of the iNOS gene abolishes estrogen-mediated protection against vascular injury in mice}

Daniela Neu, Henny Schulten, Mark Aronovitz, Flore Celestin, Richard H. Karas, and Michael E. Mendelsolun 


\begin{abstract}
Introduction - We have previously shown that estrogen inhibits the response to vascular injury in the mouse carotid arery injury model by a nitric oxide synthase-dependent pathway. In the current study, we tested the bypothesis that iNOS mediates estrogen's vascular protectiwe effecrs.

Methods - Twenty-four owariectomized, femalle wild-type (WT) and iNOS knockout $\left(\mathrm{NOS}^{-4}\right)$ mice, randomized to receive wehicle alone or $17 \mathrm{\beta}$-estradiol $\left(\mathrm{E}_{2}\right)$, were subjected to unilateral caroticl artery injury. Two weeks later, the response to vascular injury was assessed by quantification of vascular smooth muscle cell (VSMC) proliferation, measured by immunohisrochemical derection of BrdU-labeled cells (Proliferation Index, PI: BrdU-labeled cells/total cells), and medial area (MA), measured by computerized morphometry.

Resuls - Ovariectomy, estrogen teplacement or iNOS deficiency did not alter mean systolic blood pressure. BrdU-labeled cells were only rarcly detected in uninjured vessels. In the WT mice, carotid artery injury increased the PI in the vehicle-treated mice $\left(P I=0.1940 .05 ; \quad P<0.01\right.$ vs unimilured), and this was significantly attenuared by $E_{2}$ treatment ( $\mathrm{Pl}=0.10 \pm 0.03 ; \mathrm{P}<0.05$ ws $\mathrm{Wr} \mathrm{T}^{*}$ vehicle-rreated). In the $\mathrm{iNOS}^{-1-}$ mice, carotid artery injury also increased VSMC proliferation in the vehicle-treated mice $(\mathrm{PI}=0.12 \pm 0.04 ; \mathrm{P}<0.01)$, bur this was not significantly diminished in the $\mathrm{E} z$-treated mice $\left(0.12 \pm 0.02 ; \mathrm{P}=\mathrm{NS}\right.$ vs $\mathrm{NOS}^{-1 /}$ vehicle-treared). The MA of the uninjured vessels averagred $18.8 \pm 0.4 \times 10^{-3} \mathrm{~mm}^{2}$. In the WT mice, injury induced an increase in MA in whe velaide-treated animals ( $M A=26.3 \pm 0.4 \times 10^{-3} \mathrm{~mm}^{2} ; P<0.001$ vs uninjured), and this was completcly blacked in the $\mathbb{E}_{2 \text {-rreated mice }}\left(\mathrm{MA}=20 \pm 0.9 \times 10^{-3} \mathrm{~mm}^{2} ; \mathbb{P}<0.01\right.$ ws vehüle-treated). In contrast, in $\mathrm{NOS}^{-1-}$ mice, injury did not induce a significant increase in $M A$ in the velucle-rreated mice $\left(M A=20.7 \pm 1.1 \times 10^{-3} \mathrm{~mm}^{2} ; p=N S\right.$ vs uninjured), and was subsequently unaffected by $E_{2}\left(M A=20.0 \pm 0.7 \times 10^{-3} \mathrm{~mm}^{2} ; \mathrm{P}=\mathrm{NS}\right.$ us iNOS ${ }^{-1 / 2}$ vehicle-rreared).

Conduswons - The response to vascular injury is absent in mice that lack the iNOS protein. These findings identify iNOS as an important target for further investigation of the mechanisms that mediate the vascular protective effects of estrogen.
\end{abstract}




\section{INTRODUCTION}

There is currently considerable controversy, and hence considerable interest, in the cardiovascular effects of steroid sex hormones like estrogen. Several observational studies suggest that estrogen supplementation is associated with a marked reduction in cardiovascular risk in postmenopausal women. In contrast, recent randomized clinical trials have demonstrated no benefit of postmenopausal hormone replacement therapy, and, have suggested further that hormone replacement therapy may increase cardiovascular risk in some women. These discordant findings have highlighted the need for further understanding the molecular mechanisms by which these hormones affect the cardiovascular system. The cardiovascular effects of estrogen are mediated by effects on systemic, circulating factors and by direct effects on the blood vessel. 1,2-4 We have shown previously that estrogen inhibits the response to vascular injury in normocholesterolemic mice. ${ }^{5-7}$ However, the molecular mechanisms underlying this process are not well understood ${ }^{8-10}$. We recently observed that treatment of mice with the non-selective inhibitor of nitric oxide synthases, L-NAME, abolished the inhibitory effecrs of estrogen on the response to vascular injury. There are 3 known isoforms of nitric oxide synthase (NOS), and both inducible NOS (iNOS), and endothelial NOS (eNOS) have been shown to play important roles in regulating vascular biology. iNOS is generally considered to be a pro-inflammatory factor, induced in cells such as macrophages and vascular smooth muscle cells (VSMC) by cytokines and other inflammatory stimuli. "The expression of iNOS is increased in injured arteries, ${ }_{x}^{1-15}$ and the response to vascular injury is diminished in mice lacking iNOS. ${ }^{16,17}$ Taken together with previous reports demonstrating that estrogen regulates iNOS expression in mouse and rat aorta, ${ }^{18,19}$ as well as in cultured cells, ${ }^{20,21}$ these observations led us hypothesize that estrogen's vascular protective effects are in part mediated by its effects on iNOS gene expression. Therefore, as a first step to explore further the role of specific NOS isoforms in the vascular protective effects of estrogen, we examined the effects of estrogen on the vascular injury response in iNOS knockour (iNOS ${ }^{-1}$ ) mice.

\section{MATERIAL AND METHODS}

\section{ANIMALS}

All procedures involving experimental animals were performed in accordance with the Public Health Service animal welfare policy and the American Association for the Accreditation of Laboratory Animal Care. Animal protocols were approved by the Institutional Animal Care Use Committee at Tufts-New England Medical Center. Animals were housed at four per cage in a temperature-controlled facility on a 12 hour light-dark cycle, and fed regular rodent chow ad libitum. Female iNOS-1- 
mice $(n=24)$ and their respective littermate wild-type (WT) controls $(n=24)$ were obtained from Jackson Laboratories, Bar Harbor, ME. ${ }^{22}$ The genotype of all animals was confirmed by PCR analysis of genomic DNA isolated from tail snips taken at the time of harvest following the protocol provided by the vendor.

\section{BLOOD PRESSURE MEASUREMENTS}

Blood pressure was monitored in conscious animals well-acclimated to the procedure. Systolic blood pressure was measured by the tail-cuff method using a BP-2000 Specimen Platform (Visitech Systems, Cory, NC). Groups of four animals were measured over $45 \mathrm{~min}$ in intervals of $5 \mathrm{~min}$, each interval consisting of 10 measurements. Results obtained during the first 15 minutes were excluded from the final analysis and the remaining results were averaged. All measurements were done at the same time on two consecutive days by an investigator blinded to the genotype of the mice.

\section{ESTRADIOL RADIO-IMMUNOASSAY}

Circulating $\mathrm{E}_{2}$ levels at the rime of harvest were measured in triplicate from $200 \mu \mathrm{L}$ pooled serum using a commercially available radio-immunoassay kit (Estradiol RIA, Diagnostic Systems Laboratories, Inc., Webster, TX) according to the manufacturer's instructions. Control experiments confirmed the linearity of the assay between the ranges of $0.018 \mathrm{nM}$ and $2.78 \mathrm{nM}$.

\section{CAROTID ARTERY INJURY}

The carotid artery injury was performed as described previously. ${ }^{5-7,23-25}$ Briefly, thirteen week-old mice were ovariectomized and allowed two weeks to recover. They were then randomized to receive subcutaneously either vehicle alone (Veh), or 21 -day sustained release pellets containing $100 \mathrm{mg}$ of $17 \beta$-estradiol $\left(\mathrm{E}_{2}\right.$; Innovative Research of America, Sarasota, FL). One week after pellet implantation, gas-anesthetized (2.5\% Isoflurane, Abbot Laboratories, North Chicago IL) mice underwent common carotid artery injury by intraluminal passage of a fine wire, denuding the vascular endothelium and inducing a characteristic increase in medial area and VSMC proliferation. On the same day as the carotid injury, an osmotic minipump was implanted subcutaneously that releases bromodeoxy-uridine (BrdU: 25 $\mathrm{mg} / \mathrm{kg} / \mathrm{d}$ ) throughout the experiment.

\section{TISSUE HARVEST}

Fourteen days after vascular injury experimental tissues were harvested. The vasculature was perfusion fixed with $10 \%$ buffered formalin under constant pressure 
of $125 \mathrm{mmHg}$ for $4 \mathrm{~min}$. Borh common carotid arteries were then harvested, embedded in paraffin and processed as previously described, $5,7,23-25$

\section{MORPHOMETRY AND BRUU LABELING}

All morphometric analyses were made by two independent observers blinded to genotype and treatment. The response to vascular injury was assessed by 2 methods. VSMC proliferation was assessed by identifying BrdU-labeled cells by immunostaining as previously described.5-7,23-25 A Proliferation Index (PI) was calculated as the ratio of BrdU-positive VSMC to total VSMC in each section. To distinguish berween VSMC and endothelial cells, immunostaining for vascular smooth muscle cell specific actin and the endothelium specific Von Willebrand Facror was performed as described $5-7,23-25$. The response to vascular injury was also assessed by measuring vascular medial area (MA) from elastin-stained sections from each artery as described. ${ }^{5-7 \times 23-25}$ Area measurements were made using a computerized morphometric analysis system (IlmagePro Plus sofware). Medial area was defined as the area within the external elastic lamina minus the area contained within the incernal elastic lamina.

\section{DATA ANALYSIS}

One mouse (iNOS ${ }^{-1-}$ ) that developed signs off an acute ischemic stroke at the time of vascular injury was sacrificed. At time of harvest, four arteries were thrombosed (three WI vehicle-treated, one $\mathrm{NNOS}^{-1} ; \mathrm{E}_{2}$-treated). These animals were excluded from further evaluation. Furthermore, three of the injured arteries (one WT; vehicle-treated, one WT $E_{2}$-treated, one $\mathrm{NOS}^{-/}$; vehicle-treated) were excluded due to incomplete injury, as evidenced by the lack of BrdU-labeled endothelial cells.

All values are expressed as meantSEM. When only rwo groups were compared, differences were assessed by the unpaired Student's t-test. When multiple group comparisons were required, differences between the means were tested by one- or rwo-way-ANOVA. Where appropriate post-hoc analysis was performed using Student-Newman-Keuls multiple comparison test. All statistics were performed using GraphPad software. $P<0.05$ was considered to be staristically significant.

\section{RESULTS}

\section{E2 LEVELS AND UTERINE WEIGHTS}

$E_{2}$ treatment provided similar serum levels of $E_{2}$ in the WT $(0.68 \pm 0.01$ nmol/L) and $\mathrm{iNOS} / \mathrm{mice}(0.57 \pm 0.0 \mathrm{nmol} / \mathrm{L})(\mathrm{n}=8)$. In the placebo-treated animals the $\mathrm{E}_{2}$ levels were below detection. Ez treated animals had uterine weights of $9815 \mathrm{mg}$ 
$(n=24)$, while in placebo-treated animals the uterine weights were reduced to $8 \pm 0.3$ mg, $(n=23)(\mathbb{P}<0.001)$. A subgroup analysis revealed no differences in uterine weights between the WT and iNOS- animals in their respective groups (Figure 1).

\section{BLOOD PRESSURE MEASUREMENTS}

To determine whether there were differences in blood pressure between the iNOS ${ }^{-1}$ and wild type mice that might alter the response to vascular injury, arterial blood pressures were evaluated. At baseline prior to ovariectomy, mean arterial blood pressure did not differ between the WT and NOS $^{-1-}$ mice (WT, $114 \pm 3 \mathrm{mmHg}, \mathrm{n}=24$; iNOS $^{-\%}, 112 \pm 3 \mathrm{mmHg}, \mathrm{n}=23, \mathrm{P}=\mathrm{NS}$ ). Similarly, at the time of harvest, mean systolic pressure did not differ between the wild type and $\mathrm{NOS}^{-1 /}$ mice in either the placebo-treated or $\mathrm{E}_{2}$-treated groups. There was a trend towards an effect of treatment on blood pressure in that the ovariectomized placebo-treated animals of both genotypes had somewhat higher blood pressures than prior to ovariectomy or after ovariectomy with $\mathrm{E}_{2}$ treatment, but this did not reach statistical significance.

\section{RESPONSE TO VASCULAR INJURY}

BrdU-labeled cells were rarely detected in the uninjured arteries (mean $<1$ cell per section). As shown in Figure 3 , in the WT mice, carotid injury induced a pronounced increase in BrdU-labeled VSMCs in the vehicle-treated animals $\mathrm{PI}=0.19 \pm 0.05 \quad\left(\mathrm{P}<0.01\right.$ vs uninjured). $\mathrm{E}_{2}$ attenuated this increase, although not completely to the level of uninjured vessels $(\mathrm{PI}=0.10 \pm 0.03 ; \mathrm{P}<0.05$ vs uninjured; $\mathrm{P}<$ 0.05 vs WT; vehicle-treated). Vascular injury induced a similar increase in VSMC proliferation in the iNOS ${ }^{-1}$ animals $(\mathrm{PI}=0.12 \pm 0.04, \mathrm{P}<0.01$ vs uninjured, $\mathrm{P}=\mathrm{NS}$ vs injured WT; vehicle-treated). In contrast to what was found in the WT mice, $\mathbb{E}_{2}$ treatment had no effect on the injury-induced increase in VSMC proliferation in the $\mathrm{iNOS}^{-1}$ mice ( $\mathrm{PI}=0.12 \pm 0.02 ; \mathrm{P}^{\mathrm{N}}=\mathrm{NS}$ vs iNOS $^{-1}$; wehicle-treated).

In the $W_{T} T$ animals, injury significantly increased medial area (uninjured $18.8 \pm 4.1 \times 10^{-3} \mathrm{~mm}^{2}, \mathrm{n}=47$ vs WT injured vehicle-treated $26.3 \pm 3.6 \times 10^{-3} \mathrm{~mm}^{2}$, (Figure 4) $n=7, P<0.001$ ) in wild-type animals and $E_{2}$ treatment inhibited this increase in medial area (WT injured; $E_{2}$-treated $=20.82 \pm 8.91 \times 10^{-3} \mathrm{~mm}^{2}, \mathrm{n}=11, \mathrm{P}=\mathrm{NS}$ vs uninjured; vehicle-treated). In the iNOS ${ }^{-\%}$ mice, injury did not induce an increase MA in the vehicle-treated mice (uninjured $18.8 \pm 4.1 \times 10^{-3} \mathrm{~mm}^{2}, \mathrm{n}=47$ vs $20.7 \pm 1.1 \times 10^{-3}$ $\mathrm{mm}^{2}$ iNOS $^{-}$; vehicle treated, $\mathrm{n}=10, \mathrm{P}=\mathrm{NS}$ ) and was for this reason unaffected by $\mathbb{E}_{2}$ treatment (iNOS ${ }^{-1} ; E_{2}$-treated $20.0 \pm 0.7 \times 10^{-3} \mathrm{~mm}^{2}, \mathrm{n}=11, \mathrm{P}=\mathrm{NS}$ ). 

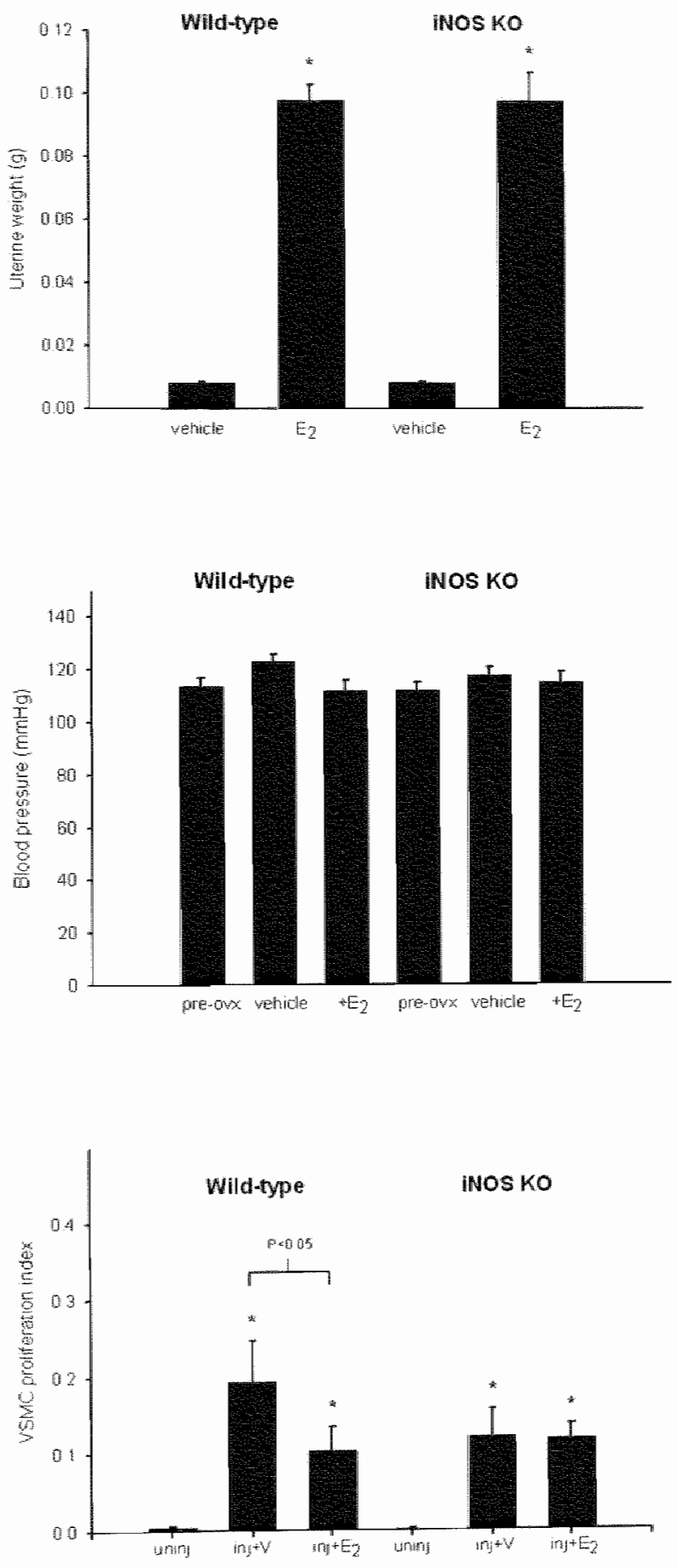

Figure 1. Estrogen increased uterine weight to a similar degree in both the WT and NOS mice. " $P<0.01$ vs vehicle-rreated animals of the same genorype. Bars represen means $\pm S E M$.

Figure 2. Summary of mean sys rolic blood pressure for all wild-rype and iNOS ${ }^{4}$ mice $(n=12+12$ and $n=11+12$, respectively) related to wehicle-treated or Ez-treated animals. There were no significant differences in mean systolic blood pressure between the groups ( $n=11-12$ per group). All dara are shown as mean \pm SEM.

Figure 3. Vascular smooth muscle cell proliferation of uninjured and injured carcitid arteries from will-rype and NOS $^{-1}$ mice treated with vehicke or 178-estradiol. Estrogen reduced VSMC proliferation $(\mathrm{P}<0.05$ vs inj+ $\mathrm{V})$. Therewere no significant differences in VSMC proliferation between wehicle-treared and $\mathbb{E}_{2}$-treated mice. " $P<0.01$ vs vehicle-treated animals of the same genotype. All data are shown as meantSEM. Uninj, uninjured; inj, irjured; $V$, vehicle, $\mathrm{E}_{2}$, 176-estradiol. 


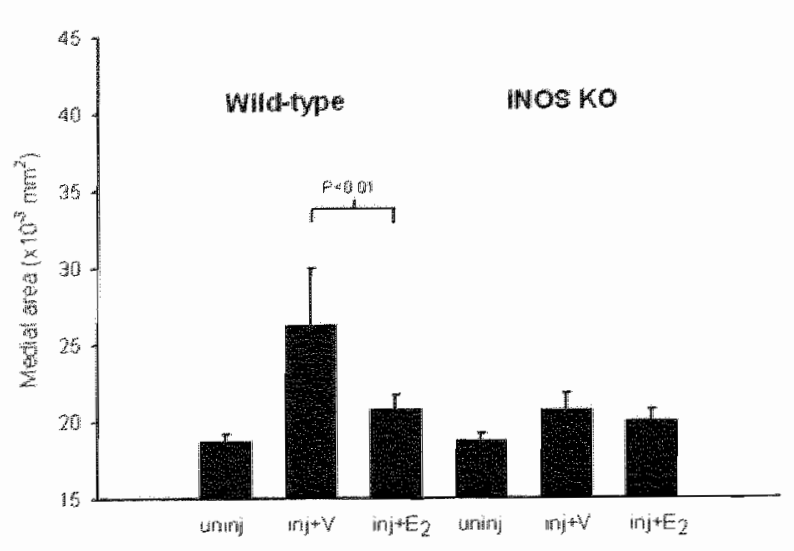

Figure 4. Medial areas of uninjwred and injured caronid arceries from wild-rype and iNOS $^{-1}$ mice treated with vehicle or 17B-estradiol. Escrogen inhibited injury winduced increases in medial area in wild-type animals ( $P=N S$, vs uning). There were no significant differences in medial areas berween the groups of iNOS ${ }^{*}$ mice. " $\mathrm{P}<0.01$ vs velicle-treated animals of the same genotype. All data are shown as meant SEM. Uning, uninjured; inj, injured; $V_{0}$ wehicle, $\mathrm{E}_{2}$, 17B-estradiol.

\section{DISCUSSION}

This study shows that $E_{2}$ inhibits vascular-injury-induced medial thickening and VSMC proliferation in ovariectomized female mice as it has been reported previously. ${ }^{5-7,23-25}$ We observed that the $\mathrm{E}_{2}$-mediated inhibition of VSMC proliferation in response to vascular injury is no longer present in NOS $^{-1-}$ mice. These results support the hyporhesis that the regulation of iNOS expression plays an important role in the $\mathrm{E}_{2}$-mediated protection against vascular injury.

To compare the results presented here with other studies it is important to identify the different models of vascular injury currently used. Each model emphasizes different aspects of the development of atherosclerosis, like the formation of neointima or vascular remodeling. ${ }^{12,26-28}$ We used a technique of carotid injury by endorthelial denudation with a wire. This model is known to lead mainlly to medial remodeling and only rarely to minimal neointima formation. ${ }^{26}$ The two endpoints of response to vascular injury that can be assessed with this model are increase in medial area and medial VSMC proliferation.

In our study the lack of iNOS expression completely blunted injury-induced medial area increase. In contrast, the deficiency of iNOS did not lead to a significant inhibition of VSMC proliferation (WT injured vs iNOS injured, $\mathbb{P}=0.25$ ), suggesting that iNOS-derived $\mathrm{NO}$ affects more the other parameters involved in medial thickening, like for example matrix formation or SMC migration from adventicia to media. However, we did not find differences in total medial cell numbers between WT and iNOS KO mice $(101 \pm 10$ vs $96 \pm 3$ respectively, $\mathrm{P}=\mathrm{NS})$. The fact that the majority of the endothelial cells stained positive for BrdU proofs that the vessels have been deendorhelialized by the wire injury to same extend as the wild-type animals. These results are in contrast to earlier studies showing that therapeutic overexpression of iNOS $^{29-32}$, has a protective effect on the response to 
vascular injury. In these studies the acute introduction of $\mathrm{NOS}$, leading to a transient boost of $\mathrm{NO}$, may have prevented the initiation of the remodeling after injury and as well as inhibition of the subsequent induction of the host's iNOS. This may be important because upregulation of the host's iNOS expression may be detrimental because this results in a prolonged production of huge quantities of $\mathrm{NO}$ leading to an inflammarory response with the subsequent induction of growth factors.

Besides this, all the currently published dara in iNOS - mice using different vascular injury models are in concordance with our findings that iNOS promotes the response to vascular injury. ${ }^{16,17}$ In regard to the study from Chyu et al ${ }^{16}$ it is important to notice that $\mathrm{INOS}$ was considered to be vasoprotective regarding the intima/media ratio. However the raw data about medial and intimal thickening confirm our data suggesting a protective role for iNOS-deficiency. "This hyporhesis is also supported by the fact that iNOS expression is induced after vascular injury ${ }^{11}$ and that $\mathrm{NOS}$ is present in atherosclerotic plaques colocalizing with oxidized LDL. which is known to have a proatherogenic effect. ${ }^{13}$ We conclude that NOS which is known to be expressed in the arterial wall after vascular injury promotes vascular remodeling by increase in extracellular matrix and proliferation of VSMC.

Several studies have demonstrated that $E_{2}$ treatment can inhibit the cytokine-induced induction of NOS in cultured VSMC and macrophage-like cells of the CNS. $18,20,21,33,34$ Furthermore, it has been shown in a model of transplant atherosclerosis that physiologic doses of $\mathrm{E}_{2}$ can inhibit the upregullation of iNOS. ${ }^{35}$ Interestingly the response to injury in the vehicle-treated iNOS deficient mice closely resembled the values in the $\mathrm{E}_{2}$-treated WT mice, and $\mathrm{E}_{2}$ treatment of the iNOS $^{-1}$ mice did not show any additional effect, suggesting that $E_{2}$ mediared downregulation of iNOS is involved in its vasculoprotective action. This parhway could also be responsible for the finding that pregnancy, which is known to be associated with elevated $\mathrm{E}_{2}$ levels, protects $\mathrm{W} T \mathrm{~T}$ as well as $\mathrm{NOS} 3-\mathrm{KO}$ mice from vascular injury, suggesting that $\mathrm{E}_{2}$ has NOS3-independent effects on vascular remodeling. ${ }^{36}$

Taken together these findings suggest that $E_{2}$ 's vasculoprotective effects are mediated by the downregulation of injury-induced iNOS expression. Further studies, using different vascular techniques and mouse models of atherosclerosis will be necessary to confirm the generall importance of these findings. In addition studies using specific iNOS inhibitors would be helpful to investigate, if these compounds mimic $E_{2}$ 's vasculoprotective effects. This is of crucial importance, since the risks associated with hormone replacement therapy have been highly publicized recently and heightened the interest in alternative therapies. ${ }^{37}$ 


\section{REFERENCES}

1. Lobo RA, Speroff L. International consensus conference on postmenopausal hormone therapy and the cardiovascular system. Fertit Sheril. 1994;61:592-5.

2. Gilligan DM, Quyyum AA, Cannon RO. 3rd. Effects of physiological levels of estrogen on coronary wasomotor funcrion in posmenopausal women. Circulation. 1994;89:2545-51.

3. Harbo J, Leth-Espensen P. Stender $\$$, Chrisciansen C. Estrogen monotherapy and combined estrogen-progestogen replacement therapy atrenuate aortic accumulation of cholesterol in ovariectomized cholesterol-fed rabbits. J Clin lnvest. 1991;87:1274-9.

4. Ehage R, Arnal JF, Pieraggi MT, Duverger N, Fiever C, Faye JC, Bayard F. 17 bera-estradiol prewents fatry streak formation in apolipoprotein E-deficient mice. Arteriosder Thromb Vast Bial. $1997 ; 17: 2679-84$.

5. Sulliwan TR Jr., Karas $\mathbb{R H}$, Aronowiz M, Faller GT, Ziar JP, Smith JJ, O. Donnell TF J, Mendelsolin ME. Estrogen inhibits the response-to-injury in a mouse carotid artery model. $\int$ Clin Invest. 1995;96:2482-8.

6. Iafrant MD, Karas RH, Aronovitz M, Kim S, Sullivan TR, Jr, Lubahn DB, O. Donmell TF J* Korach KS, Mendelsohn ME. Estrogen inhibits the vascular injury response in estrogen receptor alpha-deficient mice. Nat Med. 1997;3:545-8.

7. Karas RH, Hodgin JB. Kwoun M, Krege JH, Aronovitz M, Mackey W, Gustafsson JA, Korach KS. Smithies O, Mendelsohrn ME. Estrogen inhibits the vascular injury response in estrogen receptor beta-deficient female mice. Proc Nont Aad Sci US A. 1999;96:15133-6.

8. Grady D, Rubin SM, Petitti DB, Fox CS, Black D, Ettinger B, Ernster VL, Cummings SR. Hor mone therapy to prevent disease and prolong life in postmenopausal women. Ann Interw Med. 1992:117:1016-37.

9. Farthat MY, Lavigne MC, Ramwell PW. The wascular protective effects of estrogen. Faseb J. $1996: 10: 615-24$.

10. Mendelsohn ME, Karas RH. The protecrive effects of estrogen on the cardiovascular system. $N$ Engl/ Med. 1999;340:1801-11.

11. Hansson GK, Geng Y], Holm J, Handlammar P, Wenmmalm A, Jennische E. Arterial smooth muscle cells express nitric oxide synthase in response to endothelial injury. I Exp Med. $1994 ; 180: 733-8$.

12. Arthur JF, Yin ZL, Young HM, Dusting GJ. Inducrion of nitric oxide synthase in the neointima induced by a perarterial collar in rabbits. Arterioscler 7bromb Vasc Biol 1997;17:737-40.

13. Luoma JS, Stralin P, Marklund SL, Hiltunen TP, Sarkioja T, Ya-Hertuala S. Expression of extracellular SOD and NOS in macrophages and smooth muscle cells in human and rabbic atheroscterotic lesions: colocalization with epitopes characteristic of oxidized LDL and peroxynitrite-modified proreins. Apterioscler Tbromb Vasc Biol. 1998;18:157-67.

14. Snehr DJ, Cho HJ, Kwon NS, Weise ME, Nathan CF. Purification and characterization of the cytokine-induced macrophage nitric oxide synthase: an FAD- and FMN-containing flawoprotein. Proc Noul Acad Sci US A. 1991;88:7773-7.

15. Cohen J. Evans T], Spink J. Cyrokine regulation of inducible nitric oxide synthase in vascular smoorh muscle cells. Prog Clin Biol Res. 1998;397:169-77. 
16. Chyu KX, Dimayuga P, Zhu J, Nillsson J, Kaul S, Shah PK, Cercek B. Decreased neointimal thickening after arterial wall injury in inducible nüruc oxide synthase knockout mice. Cire Res. 1999:85:1192-8.

17. Yogo K, Shimokawa H, Funakoshi H, Kandabashi T, Myata K, Okamoto S, Egashira K, Huarng P. Akaike T, Takeshita A. Different vasculoprotective roles of NO synthase isoforms in vascular lesion formation in mice. Arterioscler. Thromb Vats Biol 2000;20:E96-E100.

18. Tamura K, Yamaguchi K, Kogo H. I7Bera-estradiol inhibirs ovariecromy-induced expression of inducible nitric oxide synthase in rat aora in vivo. Lffe Sci. 2000:66:L 259-64.

19. Zhu Y, Bian Z, Lu P, Karas RH, Bao L, Cox D, Hodgin J. Shaul PW. Thoren P. Smithies O, Gustafsson JA, Mendelsohn ME. Abnormal vascular funcrion and hypertension in mice deficient in estrogen receptor beta. Science. 2002;295:505-8.

20. Saito S, Aras RS, Lou H, Ramwell PW, Foegh ML. Effects of estrogen on mitric oxide synthase expression in rat aorta allograft and smooth muscle cells. J Heat Lung Transplant. 1999; 18:937-45.

21. Vegeto E, Bonincontro C, Pollio G, Sala A, Viappiani S, Nardi F, Brusadelli A, Viviani B, Ciana P, Maggi A. Estrogen prevents the lipopolysaccharide-induced inflammarory response in microglia. J Newrosci. 2001;21:1809-18.

22. Laubach VE, Shesely EG, Smithies $O$, Sherman PA. Mice lacking inducible nitric oxide synthase are nor resistant to lipopolysaccharide-induced death. Proc Nat Acad Sci U S A. 1995,92: $10688-92$.

23. Pare $G$, Krust A, Karas RH, Dupont S, Aronovitz M, Chambon P, Mendelsohn ME. Estrogen Receptor-alphat Mediates the Protective Effects of Estrogen Against Vascular Injury. Con Res. 2002; 90:1087-92.

24. Karas RH, Schulten H, Pare G, Aronovitz MJ, Ohlsson C, Gustafsson JA, Mendelsohn ME. EFfects of estrogen on the vascular injury response in estrogen receptor alpha, beta (double) knockout mice. Circ Res. 2001;89:534-9.

25. Lubahn DB, Moyer JS, Golding TS, Couse JF, Korach KS, Smichies O. Alteration of reproductive function but not prenatal sexual development after insertional disruption of the mouse estrogen receptor gene. Proc Natl Aad Sci U 5.A. 1993:90:11162-6.

26. Lindner V. Fingerle J, Reidy MA. Mouse model of anterial injury. Circ Res. 1993:73,792-6.

27. Kumar A, Lindner $\mathrm{V}$. Remodeling with neointima formation in the mouse carorid artery after cessation of blood flow. Anterioscler Thromb Vasc Biol 1997;17:2238-44.

28. De Meyer GR, Van Put DJ, Kockx MM, Van Schil P, Bosmans R, Bult H, Btyssens N, Vanmacele $\mathrm{R}$, Herman AG. Possible mechanisms of collar-induced intimal thickening Arterioscler Thromb Vasc Biol. 1997;17:1924-30.

29. Yan ZQ, Yokota T, Zhang W. Hansson GK. Expression of inducible nirric oxide synthase inhibits platelet adthesion and restores blood flow in the injured artery. Circ Res. 1996;79:38-44.

30. Yan $Z$, Hansson GK. Overexpression of inducible nirric oxide synthase by neointimal smooth muscle cells. Cin Ros 1998;82:21-9.

31. Tzeng E, Shears LL, 2nd, Robbins PD, Pitt BR, Geller DA, Watkins SC, Simmons RL Billiar TR. Vascular gene rransfer of the thuman inducible nitric oxide synthase: characterization of activity and effect on myointimal hyperplasia. Mol Med. 1996;2:211-25. 
32. Shears LL, 2nd, Kibbe MR, Murdock AD, Billiar TR, Lizonova A, Kovesdi I, Watkins SC. Tzeng. E. Efficient inhibition of intimal hyperplasia by adenovirus-mediated inducible nitric oxide synthase gene transfer to rats and pigs in wivo. I Am Coll Surg. 1998;187:295-306.

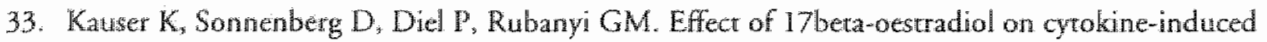
nitric oxide production in rat isolated aorta. Bry Pharnacol. 1998;123:1089-96.

34. Fimiani C, Magazine H, Welters ID, Bilfinger TV, Salsano F, Tonnesen E, Stefano GB. Antagonism of LPS and IFN anandamide, and estrogen. Acta Phorwatol Sin. 2000;21:405-9.

35. Russell ME, Wallace AF, Wyner LR, Newell JB, Karnovsky MI. Upregulation and modulation of inducible nutric oxide synthase in rat cardiac allografts with chronic rejection and transplant arteriosclarosis. Cinculation. 1995:92:457-64.

36. Moroi M, Zhang L, Yasuda T, Wirmani R, Gold HK, Fishman MC, Huang PL. Interaction of generic deficiency of endothelial nitric oxide, gender, and pregnancy in vascular response to injury in mice. J Clin Invest. 1998;101:1225-32.

37. Barrett-Connor E, Stuenkel CA. Homone replacement therapy (HRT)-risks and benefits. Int $f$ Eppidemiol. 2001:30:423-6. 
CHAPTER 6

\section{Endothelial nitric oxide synthase inhibits neointimal hyperplasia after vascular injury in hyperlipidemic mice}

Henny M. Schulten, Bianca H. Schrans-Stassen, Denny G.P. Sonnemans, Paul M.H. Schiffers, Mark J. Post, and Ebo D. de Muinck. 


\begin{abstract}
Objective - The purpose of the current study was to examine the effects of local endorthelial nirric oxide syothase (eNOS) gene transfer on neointimal hyperplasia in a byperlipidemic, atheroselerotic model. We hypothesized that in this milieu which is conducive to eNOS uncoupling and rich in superoxide, eNOS transfer alone may not inhibit neointimal hyperplasia and that is effect could be restored by adjunctive administration of folates.

Mathods and Results - ApoE $\mathrm{E}^{-/ 2}$ mice on a Western diet were subjected ro unilateral carotid artery endothelial denudaton injury and randomized inco 4 groups receiving vehicle alone, adenovirus containing $\beta$-galattosidase CDNA, adenovirus with eNOS CDNA, or the combination of adenovirus containing eNOS CDNA with folate treatment. Two weeks later, carotid anteries were harvested and the response to injury was assessed by compurerized morphometry of carotid arteries total area, medial area and incimal area. Carotid artery injury resulted in reduced intimal area and intima/media rario in mice receiving AdeNOS with or without folates, while rotal area and medial area were not affected.

Condusions - NOS reduced neointima formation after arteral injury, even in the serting of atherosclerosis and hyperlipidemia, hence folate was not required to restore its activity.
\end{abstract}




\section{INTRODUCTION}

Clinical data indicate that stents reduce postangioplasty restenosis by about $40 \%$ in spite of augmented neoincima formation. Clinically significant in-stent restenosis occurs in $20-40 \%$ of percutaneous transluminal cononary angioplasty (PTCA) cases. 2,3 In-stent restenosis is a disease characterized exclusively by neointimal hyperplasia, since the rigid device virtually precludes the recoil and constrictive remodeling that contribute to balloon angioplasty-related restenosis. Coared stents can be used as tools for local delivery of drugs or genes that inhibit neointimal hyperplasia, and this technique has opened new avenues to pursue pharmacologic or gene therapy targets that are considered unsafe when given systemically.

Although the pathophysiological mechanisms underlying neointimal hyperplasia and vascular remodeling have been studied extensively, pharmacological and mechanical approaches remain notoriously unsuccessful in mitigating the process. Several mechanisms can be identified: disruption of the endothelium, 5 plarelet activation and thrombus formation, ${ }_{3}^{6,7}$ smooth muscle cell (SMC) migration and proliferation, $5,8,9$ extracellular matrix changes and remodeling $7,10-12$ or 'shrinkage" of the artery. ${ }^{13}$ Proliferative and migratory responses of SMCs and myofibroblasts, together with extracellular matrix deposition are central to the process. These responses are driven by a variety of cytokines and chemokines, including platelet-derived growth factor, fibroblast growth factor, endothelial cell growth. factor, insulin-like growth factor, angiotensin II, and endothelin. ${ }^{14}$

The normal protective barrier function of endothelial cells is disrupted after PTCA resulring in the loss of constitutively expressed endorhelium-derived vasoactive factors, including nitric oxide (NO), prostacyclin, and bradykinin. ${ }^{5} \mathrm{NO}$ stimulates soluble guanylate cyclase in the SMC which leads to vasorelaxation, inhibition of cell proliferation and migration, and extracellular matrix production. 16,17 NO also affects circularing platelets and inhibits platelet adhesion to the vessel wall. In animal models of arterial injury, administration of $\mathrm{NO}$-donor compounds or stimulation of endogenous NO production by administration of its precursor L-arginine reduces the vascular response. ${ }^{18,19}$

eNOS consists of a flavin-containing reductase domain and a heme-containing oxidase domain. ${ }^{20}$ In the presence of $\mathrm{Ca}^{2+} /$ calmodulin, an electron is transferred from NADPH to the heme group, leading to reduction of oxygen, which in then used to oxidize L-arginine to $\mathrm{NO}$ and citrulline. BH, is an eNOS cofactor that directs the alectron flow to L-arginine. Under certain pathophysiological conditions, such as oxidative stress in hyperlipidemia, depletion of $\mathrm{BH}_{4}$ wesults in uncoupling of oxygen reduction and $\mathrm{L}$-arginine oxidation, ${ }^{21}$ thereby generating superoxide. 22 L-5-methyltetrahydrofolate (L-5-MTHF, the main form of folate in the circulation $^{23}$ ) reverses this uncoupling of eNOS supposedly by regeneration of $\mathrm{BH}_{4}{ }^{24}$

Intravascular NOS gene transfer at the site of balloon dilation or stent implantation has been shown to reduce neointimal hyperplasia in normal arteries of 
non-hyperlipidemic animals. $25-28$ However, NOS gene transfer in atheroscleromic arteries of hypedipidemic anmals could lead to uncoupling of eNOS enzymatic activity. Hence, we studied the eficacy of NOS gene transfer under conditions that could be conducive to eNOS uncoupling and the potential rescue by exogenous folate suppletion. We performed these studies in the carotid artery injury model in apolipoprotein (apo) E knockout mice.

\section{MATERIAL AND METHODS}

\section{ANIMALS}

Procedures used in these experiments were approved by the University of Maastricht Animal Care and Ethical Commitree. We studied 12 months old male homozygous apoE-deficient mice (with a $129 \times \mathrm{C} 57 \mathrm{BL} / 6 \mathrm{~J}$ background and backcrossed through 9 generations on a C57BL/61 background; Chatles River Laboratories, the Netherlands). Mice had free access to tap water and after weaning were maintained on a high fat Western diet deficient of folic acid $15 \%$ cacao butter, $2 \%$ cholate, $0.25 \%$ cholesterol, $40.5 \%$ sucrose, $10 \%$ corn starch, $1 \%$ corn oil and $5.95 \%$ cellulose; Hope Farms, Woerden, the Netherlands). One week prior to injury, animals which were randomized to receive the combination AdeNOS and folate were changed to high fat diet containing L-5-methyltetrahydrofolate (L-5-MTHF) and received supplementary L-5-MTHF in drinking water $(1 \%$, w/v).

\section{LIPID PROFILE AND L-5-MTHF DETERMINATIONS}

Plasma cholesterol and triglyceride levels were determined in duplicate by using a colonmetric assay (CHOD-PAP 1442341 and GPO-PAP 701912, respectively, La Roche) on arterial plasma samples obtained at 12 months of age.

Plasma L-5-MTHF levels were measured by fluorescence after deproteinization with perchloric acid and separation by high-performance liquid chromatography $\left(\right.$ HPLC) ${ }^{29}$

Plasma prostaglandin $\mathrm{F}_{2 \alpha}\left(\mathrm{PGF}_{2 \alpha}\right)$ levels were determined by using a competitive enzyme immunoassay according to the manufacturer's instructions (Cayman Chemicals).

\section{CAROTID ARTERY INJURY AND APPLICATION OE VIRAL CONSTRUCTS}

Sixty-five apoE- mice were anesthetized with $2.5 \%$ isoflurane and subsequently subjected to unilateral carotid artery injury as described by Lindner. 30 Briefly, the left carotid artery was exposed via a midline neck incision. The bifurcation of the carotid artery was located and two ligatures were placed around the external carotid 
artery, which was subsequently tied off with the distal ligature and the internal carotid artery was temporarily clipped off to prevent back bleeding. A puncture was made between the ligatures to introduce a curved flexible wire $(0.35 \mathrm{~mm}$ diameter, Cooke, Bloomington, IN, USA) into the common carotid artery and was passed along the vessel three times while rotating. After the wire had been removed, the common carotid artery was flushed several times with saline to get rid of debris (loose endothelial cells) and remaining blood cells. Then, a catherer was introduced into the common carotid artery and $10 \mu \mathrm{L}$ of vehicle (saline), $10 \mu \mathrm{L} 5.0 \times 10^{6} \mathrm{pfu} / \mu \mathrm{L}$ of Adlac Z or AdeNOS was instilled for 20 minutes and rinsed out. Flow was restored, the external carotid artery ligated and the skin wound closed with silk surures.

\section{TISSUE HARVEST}

Two weeks following vascular injury, the mice were anestherized again with $2.5 \%$ isoflurane and blood was drawn for cholesterol, triglyceride and L-5-MTHF determinations. Both carotid arteries were harvested after perfusion fixation with $10 \%$ formalin at $150 \mathrm{mmHg}$ for 4 minutes.

\section{HISTOLOGY AND MORPHOMETRY}

Following embedding in paraffin, parallel cross-sections $(4-\mu \mathrm{m})$ from the carotid arteries were stained with Lawson's solution (Boom BV, Meppel, the Nerherlands), which highlights the elastic membranes. Intimal, medial and total areas were determined on serial sections using Image Pro Plus software. The intimal surface area was calculated by subtracting the free lumen area from the area circumscribed by the internal elastic lamina, whereas the medial area was defined as the area between the internal lamina and the external lamina. The intima/media ratio was determined by dividing the intimal area by the medial area. Total area was defined as the area circumscribed by the external lamina. All observations were made by an observer blinded to treatment group.

For histochemical detection of $\beta$-galactosidase activity an AdLac $Z$ transfected carotid artery was harvested two days after vascular injury and placed in a solution containing $5 \mathrm{mM}$ potassium ferrocyanide, $5 \mathrm{mM}$ potassium ferricyanide, $2 \mathrm{mM}$ $\mathrm{MgCl}_{2}$ and $1 \mathrm{mg} / \mathrm{mL}$-bromo-4a-chloro-3-indolyl- $\beta$-D-galactopyranoside (X-gal) for overnight incubation at $37^{\circ} \mathrm{C}$. After fixation in $10 \%$ formalin and embedding in paraffin, 4- $\mu \mathrm{m}$ cross-sections were examined under a microscope for blue-stained cells.

\section{DATA ANALYSIS}

Results are shown as means \pm SEM. Statistical significance of differences between experimental groups were evaluated using analysis of variance (ANOVA) followed by a 
post-hoc comparison with LSD Fischer significant difference test. All staristics were performed using SigmaStat sofiware. A P-value of $<0.05$ was considered significant.

\section{RESULTS}

\section{GENERAL OBSERVATIONS}

Body weight, plasma cholesterol and triglyceride levels did not differ between experimental groups (Table 1). Mice on folates, i.e. 1\% L-5-MTHF into the drinking water and folic acid supplemented chow, had elevated L-5-MTHF-plasma levels as compared to animals on tap water and folic acid free chow. Folate treatment did not influence plasma cholesterol and triglyceride levels in the animals (Table 1). Furthermore, folate administration did not affect plasma levels of prostaglandin $\mathrm{F}_{2 \alpha}$, a biomarker for oxidative stress. Peri-operative and follow-up mortality rates were high in all groups (Table 1).

To confirm effective transduction of the viral vector containing $\beta$-galactosidase, we first examined X-gal-stained sections of the transduced injured carotid artery harvested two days after mechanical denudation (Figure 1). Expression of $\beta$-galactosidase activity, as reflected by blue staining, was observed in the intimal and medial surface and some scattered blue spots were seen in the adventitia.

\section{RESPONSE TO VASCULAR INJURY}

Uninjured carotid arteries showed minimal intimal thickening $\left(3.3 \pm 1.9 \times 10^{-3}\right.$ $\mathrm{mm}^{2}$ ). Unilateral carotid artery injury resulted in an expected increase in total vessel area as compared to uninjured vessels, however, eNOS treatment alone or in combination with folates did not affect this increase (Table 2).

Table 1. Chatacteristics of mice

\begin{tabular}{|c|c|c|c|c|}
\hline & Vehide & $\beta-g r n l$ & NOS & eNOS+folare \\
\hline Weights (g) & $38 \pm 3$ & $33 \pm 1$ & $35 \pm 2$ & $34 \pm 2$ \\
\hline Cholesterol (mmol/L) & $24: 9$ & $20 \pm 3$ & $21 \pm 3$ & $18 \pm 4$ \\
\hline Triglycerides (mmol/L) & $.56 \pm .20$ & $.41 \pm .05$ & $.57 \pm 0.07$ & $.54 \pm .01$ \\
\hline L-5-MTHH: (nmol/L) & $76 \pm 18$ & $118 \pm 48$ & N.D. & $977 \pm 139^{*}$ \\
\hline $\mathrm{PGF}_{2 a}(\mathrm{ng} / \mathrm{m})$ & $3.1 \pm 3$ & $3.0 \pm .6$ & $3.0 \pm 3$ & $3.2 \pm .3$ \\
\hline Montality (\%) & 53 & 62 & 36 & 62 \\
\hline
\end{tabular}

Values are means $\pm E M(n=5-9)$. N. D not decectable. ${ }^{*} p<0,001$ ws vehicle, $\beta$-ged and eNOS. 
Table 2. Effect of trearment on vessel wall morphometry

\begin{tabular}{|c|c|c|c|c|}
\hline Treatment & $\begin{array}{l}\text { NI area } \\
\left(\mathrm{x} 10^{-3} \mathrm{~mm}^{2}\right)\end{array}$ & $\begin{array}{l}\text { Medial area } \\
\left(\mathrm{k} 10^{-9} \mathrm{~mm}^{2}\right)\end{array}$ & UM ravio & $\begin{array}{l}\text { Total area } \\
\left(x 10^{-3} \mathrm{~mm}^{2}\right)\end{array}$ \\
\hline Uninjured & $3.3 \pm 1.9$ & $23.3 \pm 1.0$ & $.05 \pm .03$ & $78.1 \pm 4.5$ \\
\hline Injured, vehicle & $39.7 \pm 13.9 *$ & $46.6 \pm 3.9^{*}$ & $64 \pm 21 *$ & $133.5 \pm 7.5 *$ \\
\hline Injured, $\beta$ gal & $31.2 \pm 11.3^{*}$ & $44.9 \pm 4.4 *$ & $49 \pm 23^{*}$ & $153.8 \pm 8.0$ * \\
\hline Injured, eNOS & $12.6 \pm 3.2+$ & $50.6 \pm 3.5 *$ & $.22 \pm .05+$ & $133.8 \pm 6.1 \%$ \\
\hline Injured, eNOS folate & $17.3 \pm 7.0+$ & $52.3 \pm 5.8 *$ & $.23 \pm .05 \dagger$ & $146.1+8.5 *$ \\
\hline
\end{tabular}

Values are means \pm SEM $(n=5-9)$. NI neoinuma, ${ }^{*} p<0.05$ vs uningured, $+p<0.05$ ws velaide.

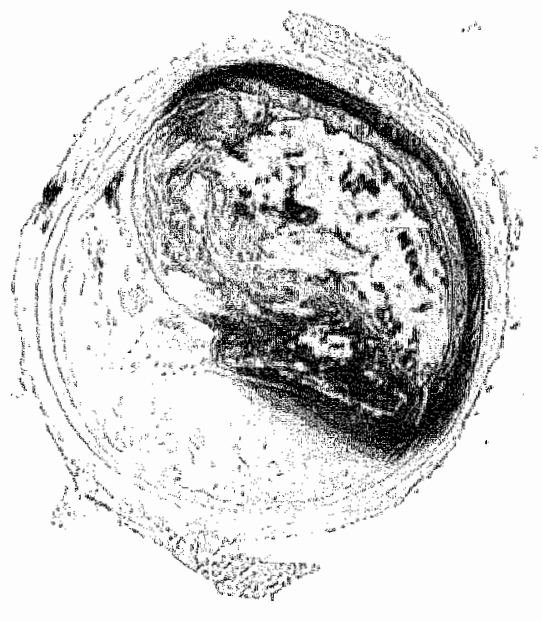

Figure 1. B-gal expression in an injured carotid altery of an apoE- monse two days after gene transfer and carotid artey denudacion. Transgene expression is present in neointina, medial area and rarely spotted in the adventitia. Sections were counterstained with nuclear fast red.

Neointima formation was significantly inhibited by $68 \%$ in animals treated with eNOS gene transfer, and by $56 \%$ in animals treated with AdeNOS and L-5-MTHF (Figures 2, 3 and Table 2). Vascular injury resulted in significant increases in carotid medial area in animals receiving vehicle alone $\left(46.6 \pm 3.9 \times 10^{-3} \mathrm{~mm}^{2}\right), \beta$-galactosidase $\left(44.9 \pm 4.4 \times 10^{-3} \mathrm{~mm}^{2}\right)$, eNOS $\left(50.6 \pm 3.5 \times 10^{-3} \mathrm{~mm}^{2}\right)$ or the combination of eNOS and L-5-MTHF/folic acid $\left(52.3 \pm 5.8 \times 10^{-3} \mathrm{~mm}^{2}\right)$ as compared to the uninjured vessels $\left(23.3 \pm 1.0 \times 10^{-3} \mathrm{~mm}^{2}\right)$ (Figure 2,4 and Table 2$)$. Unexpectedly, and in spite of the robust medial thickening by injury, medial area was not affected by treatment. To correct for initial changes in arterial size, incima/media (I/M) ratios were calculated (Figure 5 and Table 2). AdeNOS alone or with supplemental L-5-MTHF/folic acid significantly reduced intima/media ratios of injured vessels. 
A

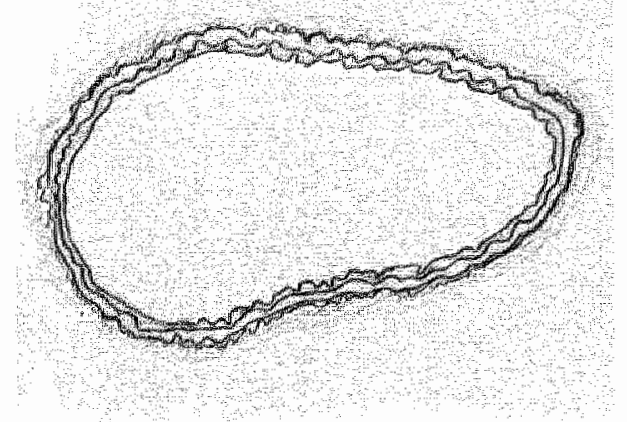

C

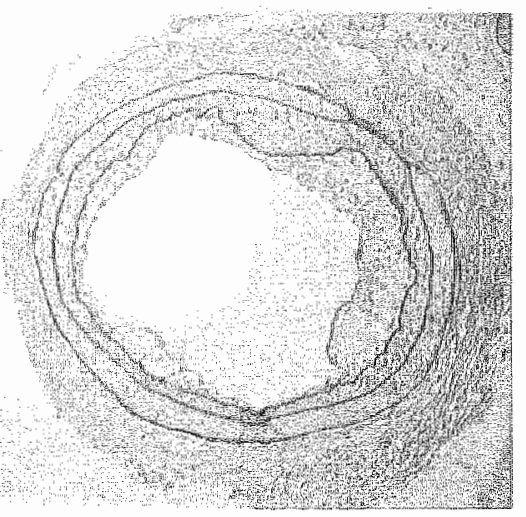

8.
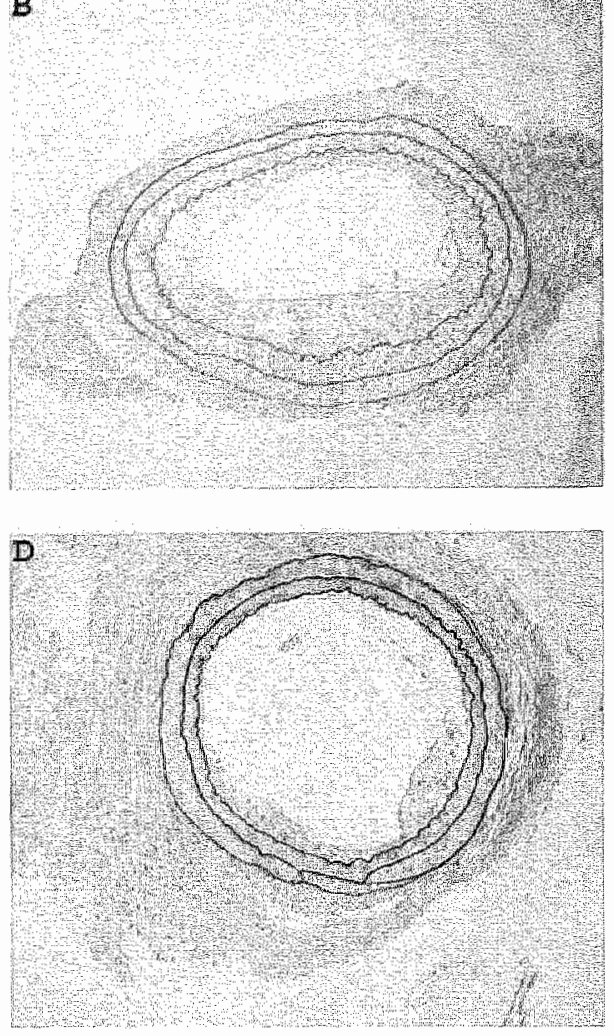

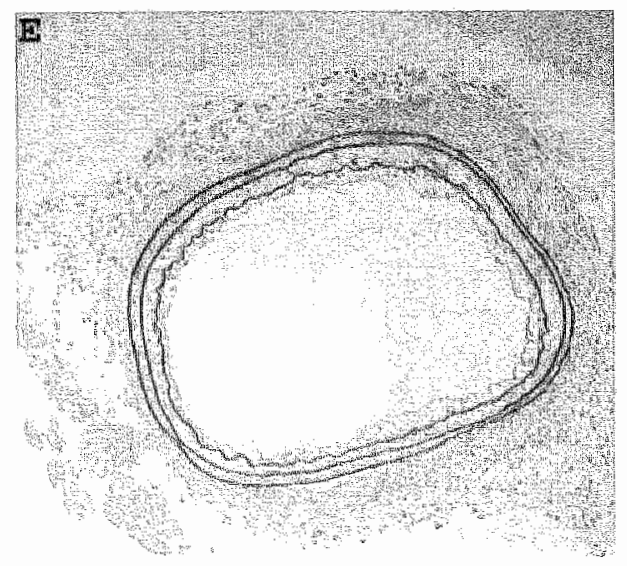

Figure 2. Representative sections stained for measurement of neoincimal, medial and total area. Neointimal areas of uninjured and injured mice treared with vehicle, $\beta$-gat cDNA gene transfer, eNOS CDNA gene ransfer or in combinarion with systemic folate administration. (A-E) Representative segmenrs from elascin-srained carotid artery sections are shown. All area measurements were made on complere sections. (A) uninjured, (B) injured, vehicle-treated, (C) injured, adeno $\beta$-gat-rreated, (O) injured, adeno NOS-treated, (C) injured, adeno eNOS-treated + systemic folate administration (one week prior ro injury). 

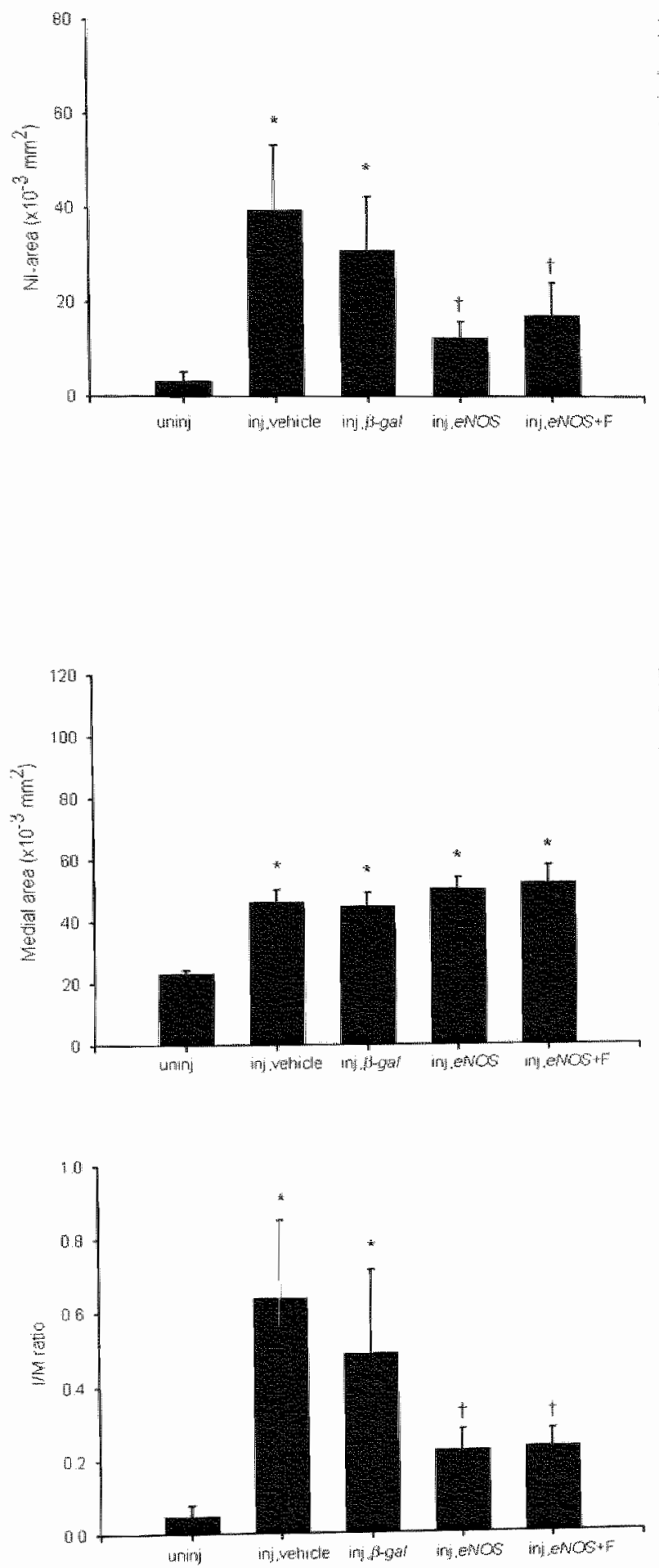

Figure 3 . Neoinrimal area in uninjured (minj) and injured (inj) carotid arteries hom mice receiving vehicle (ini, vehide), adeno $\beta-$ gat cDNA (inj, B-gath), adeno eNOS cDNA (mi. NOS or in combination with systemic folate $(F)$ administration (inj, NOS+F). eNOS gene ranster, and in combination with folate administration (one week prior to injury) signiticantly inhibited the injury-induced increase in neointimal atea ro levels comparable to the uninjured animals. The neointinal area was determined by computerized morphometric analysis of full cincumference secrions from each animal. Bars represent the mean $\pm S E M$ for each group. " $P<0.05$ vs uninjured, $+P<0.05$ ws velaicle.

Figure 4. Medial area in uninjured (uninj) and injured (inj) carotid arteries from mice receiving vehicle (inj, vehicle), adeno $\beta-g a /$ DNA (inj, $\beta-g a l)$, adeno eNOS CDNA (inj, eNOS) or in combination with systemic folate (F) administration (inj, eNOS+Fa). Treatment did not inhibir the injury-induced increase in medial area. The medial area was determined by computerized morphometric analysis of Full circumference sections from each animal. Bars represen the mean 4 SEM for each group. "P<0.05 vs uniniured.

Figure 5. Intima/modia ratio in unirnjured (uninj) and injured (inj) carotid arteries from mice receiving vehiclc (inj, velicle), adeno $\beta$-gal cDNA (inj, B-gal), adeno eNOS CDNA (inj. NOS) or in combination with systemic folate (F) administration (inj. eNOS+ $F$ ). I/M ratio was significantly reduced in animals treated with eNOS or in combination wits folate administration (one week prior ro injury). Bars represent the meantSEM for each group. * $\mathbb{P}<0.05$ vs uninjured, $+P<0.05$ vs velside. 


\section{DISCUSSION}

In this study in hyperlipidemic atherosclerotic mice we showed that local adenoviral gene transfer of NOS reduces neointima formation after arterial injury robustly so that additional benefit of adjuvant folic acid treatment could not be ascertained. This result is in accordance with $N O$ suppletion studies in non-atherosclerotic models of arterial injury $18,19,25-28$ and has important dinical implications in that it shows the efficacy of $N O S$ gene ransfer in an environment conducive to uncoupling of eNOS function and generation of excess superoxide, i.e. the environment in which percutaneous interventions typically are performed.

VSMC proliferation and macrophage activation at the site of injury are central to the remodelling after angioplasty ${ }^{6,7}$ and both are strongly affected by NO. NO inhibits VSMC growth in virro and experimental neointimal hyperplasia in wivo, indicating a role for $\mathrm{NO}$ as a regulator of VSMC proliferation. ${ }^{16,17} \mathrm{NO}$ is also involved in the control of several other pathophysiological responses, including platelet function and inflammatory cell adhesion, vascular reactivicy, and endothelial permeability. 16,17 Local delivery of the $\mathrm{NO}$ donor molsidomine improved hemodynamics, lowered cell proliferation and prevented late lumen loss in femoral arteries of atherosclerotic pigs after balloon angioplasty ${ }^{31}$ Likewise, systemic delivery of NO by a NO-releasing aspirin showed decreased neointimal hyperplasia after vascular injury in hyperlipidemic ${ }^{32.33}$ and non-hyperlipidemic ${ }^{19}$ rodents. Thus, a large body of data supports the rationale for augmenting $\mathrm{NO}$ availability as a strategy to prevent neointimal hyperplasia. In the presence of atherosclerosis, NO suppletion remains effective, although in a large clinical study, nitric oxide suppletion by linsidomine followed by oral molsidomine did not affect restenosis rates after balloon angioplasty (measured as 'loss index') in patients with single vessel coronary disease. $^{34}$

Exogenous administration of endothelial $\mathrm{NO}$ synthase is an alternative and possibly more controlled way to deliver $\mathrm{NO}$ to the vascular wall. The proof of concept was provided by studies on intra-arterial adenoviral NOS transfer that showed a $70 \%$ inhibition of neoincima formation in the rat carotid artery balloon injury model. 26,28 We know of no other studies however, that tested the efficacy of NOS suppletion in the setting of hypercholesterolemia and there are reasons to believe that the enzymatic activity of NOS is compromised under condition of augmented oxidative stress.

In hypercholesterolemia with elevated vascular superoxide $\left(\mathrm{O}_{2}\right.$-) production by increased expression of xanthine oxidases, $\mathrm{NO}$ combines with $\mathrm{O}_{2}$ - to form peroxynitrite, thereby reducing the bioavailability of NO. Furthermore, the formed peroxynitrite oxidizes tetrahydrobiopterin $\left(\mathrm{BH}_{4}\right)$, a folic acid compound, causing the uncoupling of NO synthase (uncoupling of $\mathrm{NADPH}$ oxidation and $\mathrm{NO}$ synthase). Isoprostanes, like prostaglandin $\mathrm{F}_{2 \alpha}$, are chemically stable prostaglandin isomers that result from oxidative modification of arachnidonic acid through a 
mechanism catalyzed by free radicals. ${ }^{35}$ These isoprostanes acumulate in human atherosclerotic plaque, ${ }^{36}$ a tissue in which oxidative stress with consequent eNOS uncoupling is thought to occur. In our study, we used the apoE deficient mouse as a model of hypercholesterolemia and atherosclerosis. Plasma levels of prostaglandin $\mathrm{F}_{2 \alpha}$ were higher in this model compared to age-matched $\mathrm{C} 57 \mathrm{BL} / 6 \mathrm{~J}$ mice, even on a normal chow diet. ${ }^{37}$ In the older apoE deficient mice on a high cholesterol diet that were used in the present study, $\mathrm{PGF}_{2 \alpha}$ levels were even higher than in these historic controls, thus providing evidence for augmented oxidative stress.

In spite of elevated oxidative stress, eNOS transfection still effectively blocked neointima formation, suggesting that uncoupling of the enzyme either does not occur in this model or is not sufficient to block its effect. The observation that folate addition did not increase the effect of eNOS suppletion, further supports absence of uncoupling in this system through $\mathrm{BH}_{4}$ oxidation. Vascular superoxide production appears to be ar least partially mediated by $\mathrm{BH}_{4}$-dependent eNOS uncoupling in apoE deficient mice ${ }^{38}$ so it was to be expected that folate administration would lower vascular superoxide and systemic $\mathrm{PGF}_{2 \alpha}$ levels. However, in our study, folate suppletion did nor lower plasma $\mathrm{PGF}_{2 \alpha}$ levels, indicating that the oxidative stress status of the animal remained unchanged suggesting that uncoupling of eNOS if present, did not contribute to the vascular pool of superoxide. Other oxidase systems like, NAD(P)H and xanthine oxidases are also active in the formation of ROS (including superoxide) so uncoupling of eNOS is definitely not required to increase oxidative stress in the vascular wall. It is conceivable, that exogenous eNOS for instance transfected into wascular smooth muscle cells may nor be subject to uncoupling, or to increased oxidative stress. The smooth muscle cell milieu, as compared to that of the endothelial cell, may differ in oxidative stress level (less) or differ in other factors that may alter eNOS enzymatic activity. For example, hypercholesterolemia-induced caveolin-mediated inhibition of eNOS activity in endothelial cells ${ }^{39}$ may not occur in smooth muscle cells as these cells lack caveolin. The general, not endothelial specific, antioxidant acrivity of L-5-MTHF, ${ }^{40,41}$ is apparendy also not strong enough to reduce neointima formation. Recently, two studies revealed that long term vitamin $\mathrm{E}$ and $\mathrm{C}$ treatment could reduce atherosclerosis by reducing oxidation in apoE deficient mice. ${ }^{37,42}$ The antioxidant potency of L-5-MTHF was reported to be about 20 -fold lower than the scavenging effects of vitamin $\mathrm{C}$, therefore the relevance of a direct antioxidant effect of folate is probably limited. This was supported by a failure of folates to lower plasma isoprostane level reduction in this study.

Recent evidence suggests thar uncoupling of eNOS may not be folate-dependent bur related to peroxynitrite's ability to disrupt the zinc-thiolate cluster of eNOS enzyme. Zou et al ${ }^{43}$ reported peroxynitrite-induced uncoupling of purified eNOS by disrupting the zinc-thiolate cluster of the enzyme while the essential cofactor $\mathrm{BH}_{4}$ was not oxidized. Addition of high concentrations $(0.1 \mathrm{mmol} / \mathrm{l})$ of $\mathrm{BH}_{4}$ did not 
result in preventing eNOS from oxidation by low concentrations $(50 \mu \mathrm{mol} / \mathrm{l})$ of peroxynitrite.

\section{GENERAL CONCLUSIONS}

The current study is the first to demonstrate effective inhibition of neointimal hyperplasia after local vascular gene transfer of eNOS in a hyperlipidemic, atherosclerotic model. The maintained efficacy of the enzyme despite its transfer into an environment conducive to uncoupling of eNOS function and rich in superoxide remains unexplained. However, the clinical implications of these findings are that $e N O S$ transfer after stent implantation using the stent as a delivery platform, may prove an effective strategy to prevent in-stent restenosis.

\section{ACKNOWLEDGEMENT}

We kindly want to thank Rudolf Moser for providing L-5-MTHF. Jet Bost is greatly appreciated for excellent technical assistance.

\section{REFERENCES}

1. Fisech M, Meier B. Management and outcome of stents in 1998: long-term outcome. Cardiol Rev. 1999;7:215-218.

2. Bauters C, Banos JL, Van Belle E, Mc Fadden EP, Lablanche JM, Bertrand ME. Six-month allgiographic outcome after successful repear percutaneous intervention for in-stent restenosis. Circutarion. 1.998,97:318-321.

3. Yutam C. Imakita M. Ishibashi-Ueda $H$, Tsukamoto $Y$, Nishida $N$, Ikeda Y. Coronary atherosclerosis and interwentions: pathological sequences and restenosis. Pathol Int 1999;49:273-290.

4. Post MJ, de Smer BJ, wan der Helm Y, Borse C, Kunz RE. Arterial remodeling after balloon angioplasty or stencing in an atherosclerotic experimental model. Circulation. 1997;96:996-1003.

5. Cohen RA. The role of nitric oxide and other endorhelium-derived wasoactive substances in vascular discase. Prog Cardiouase Dis. 1995;38:105-128.

6. Casscells W. Smooth muscle cell growrh factors. Prog Growatb Fatcor Res. 1991;3:177-206.

7. Schwarz RS, Holmes DR, Ir., Topol EJ. The restenosis paradigm revisited: an altremarive proposal for cellular medranisms. IAm Coll Cardiol. 1992;20:1284 1293.

8. Jackson CL, Raines EW, Ross R, Reidy MA. Role of endogenous plateler-derived growth factor in arterial smooth muscle cell migration after balloon catheter injury. Arteriosclep Thromb. $1993 ; 13: 1218-1226$. 
9. Miano JM, Masic N, Tora RR, Stemerman MB. Smooth muscle cell immediate-early gene and growth factor activation follows vascular injury. A purative in wiwo mechanism for tutocrine growth. Aneriosder Thromb. 1993; 13:211-219.

10. Strauss BH, Umans VA, wan Suylen R., de Feyter P], Marco J, Robertson GC, Renkin J, Heyndrickx $G$, Vuzevski VD, Bosman FT, er al. Directional atherectomy for treament of restenosis within coronary stents: clinical, angiographic and histologic resules. / Am Coll Cortaol. 1992;20:1465-1473.

11. Nobuyoshi $\mathbb{M}$, Kimura T, Ohishi $H$, Horiuchi $H$, Nosaka $H$, Hamasaki $\mathbb{N}$, Yokoi $\mathbb{H}$, Kim $K$. Restenosis after percutaneous transluminal coronary angioplascy: pathologic obserwations in 20 paciens. J Am Coll Candiol 1991;17:433-439.

12. Coars WD, Jr, Whittaker P, Cheung DT, Currier IW, Han B, Faxon DP. Collagen content is significantly lower in restenonic versus nonrestenotic yessels after balloon angroplasty in the atherosclerotic rabbir model. Circulation. 1997;95:1293-1300.

13. Kimura T, Kaburagi S, Tamura T, Yokoi H, Nakagawa $Y$, Hamasaki N, Nosaka H, Nobuyoshi M. Mintz GS, Popma J], Leon. MB. Remodeling of human coronary arreries undergoing coronary angioplasty or atherecromy. Cimculation. $1997: 96: 475-4: 83$.

14. Schwartz SM, deBlois D, O'Brien ER. The intima. Soil for atheroselerosis and restenosis. Circ Res. $1995 ; 77: 445-465$.

15. Lloyd-Jones DM, Bloch $\mathrm{KD}$. The vascular biology of nitric oxide and its role in atherogenesis. Annu Rev Med. 1996:47:365-375.

16. Moncada S, Palmer RM, Higgs EA. Nitric oxiden physiology, pathophysiology, and pharmacology. Pharracal Rev. 1991;43:109-142.

17. Nathan C. Xie QW. Nitric oxide synthases: roles, rolls, and controls. Cell. 1994;78:915-918.

18. Kalinowski M, Alfke $H$, Bergen S, Klose KJ, Barry JJ, Wagner HJ. Compararive crial of local pharmacotherapy with L-arginine, $r$-hirudin, and molsidomine to reduce restenosis after balloon angioplasty of stenotic rabbit iliac anteries. Radiology. 2001;219:716-723.

19. Maffia P, Ianaro A, Sorrentino R, Lippolis L, Maiello FM, del Soldato P, Lalenci A, Cirno G. Beneficial effecs of $\mathrm{NO}$-releasing derivative of Hurbiprofen (HCT-1026) in ra model of valscular injury and restenosis Arterioscler Thromb Var Biol. 2002:22:263-267.

20. Forstermann U, Closs EI, Pollock IS, Nakane M, Sclhwarz P, Gath I, Kleinert H. Nitric oxidc synthase isorymes. Charactertation, purification, molecular cloning, and functions. Hyprentonion. $1994: 23: 1121-1131$.

21. Gorren AC, List BM, Schrammal A, Pitters E, Hemmens B, Werrer ER, Schmidt K, Mayer B. Terrahydrobiopterin-free neuronal wirric axide synthase: evidence for wo idenrical highly anticooperative preridine binding sires. Biochewhstry. 1996,35:16735-16745.

22. Pou $S$, Keaton L, Surichamorn W, Rosen GM. Mechanism of superoxide generation by neuronal mitric-oxide synthase./Btol Chem. 1999:274:9573-9580.

23. Loehrer FM, Angst CP, Haefeli WE, Jordan PP, Ritz R, Fowler B. Low whole-blood S-adenosylmethionine and correlation between 5 -methylterahydrofolate and homocysteine in coronary arrery disease. Areerioncler Thromb Vasc Biol 1996;16:727-733.

24. Kaufman $S$. Some metabolic relationships between biopterin and folate: implications for the "raethyl rap hypothesis". Neworbem Res. 1991:16:1031-1036. 
25. Fang S, Sharma RW, Bhalla RC. Enhanced recovery of injury-caused downregulation of paxillin protein by cNOS gene expression in rat carotid artery. Mechanism of NO inhibition of intimal hyperplasia: Arterioster. Thromb Vocs Biol 1999:19:147-152.

26. Janssens $S$, Flaherry $D$, Nong $Z$, Varenne $O$, wan Pelt $N$, Haustemans $C$, Zoldhelyi $P$, Gerard $R_{\text {. }}$ Collen $\mathrm{D}$. Human endothelial nirric oxide synthase gene transfer inhibits wascular smooth muscle cell proliferation and neointima formation after balloon injury in rats. Circtlation. $1998: 97: 1274-1281$.

27. Varenne O, Pistaru S, Gillijns H. Van Pelt N, Gerard RD, Zoldhely: P, Van de Werf F, Collen D, Janssens SP. Local adenovirus-mediated transfer of human endothelial nitric oxide synthase veduces luminal nartowing after coronary angioplasty in pigs. Circulation. 1998;98:919-926.

28. von der Leyen HE, Gibbons GH, Morishita R, Lewis NP, Zhang L, Nakajima M, Kaneda Y, Cooke JP. Dzau VI. Gene therapy inhibiting neointimal vascular lesion: in wivo transfer of endothelial cell nitric oxide synthase gene. Proc Nat Aond So U S A. 1995;92:1137-11.41.

29. Leeming RJ, Pollock A, Melville L.j. Hamon CG. Meastirement of 5-merhylterrahydrofolic acid in man by high-performance liquid chromatography. Metabolism. 1990;39:902-904.

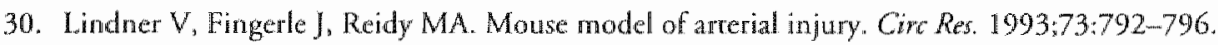

31. Rolland PH, Bartoli JM, Piquet P. Mekkaoui C, Nott SH, Moulin G, Amabile P, Mesana T. Local delivery of NO-donor molsidomine post-PTA improves haemodynamics, wall mechanics and histomorphometry in atherosderoric porcine SFA. Eur J Vasc Endovasc Surg. 2002;23:226-233.

32. Yul, Rudic RD, Sessa WC. Nitric oxide-releasing aspinin decreases vascular injury by reducing inflammation and promoting apoptosis. Lab Invest. 2002;82:825-832.

33. Napoli C, Cirino G, Del Soldato P, Sorrentino R, Sica V, Condorelli M, Pinto A, Ignarro LJ. Effects of nitric oxide-releasing aspirin versus aspirin on restenosis in hypercholesterolemic mice. Proc Natl Acad Sci US A. 2001;98:2860-2864.

34. Lablanche JM, Grollier G, Lusson JR, Bassand JP, Drobinski G, Berrand B, Battaglia S, Desveaux B, Juilliere Y, Juhlard JM, Metzger JP, Coste P, Quiret JC, Dubois-Rande JL, Crochet PD, Letac B. Boschat J. Virot P. Finer G, Le Breton H, Livarek B, Leclercq F, Beard T, Giraud T, Bertrand ME, at. Effect of the direct nitric oxide donors linsidomine and molsidomine on angiographic restenosis afrer coronary balloon angioplasty. The ACCORD Study. Angioplastic Coronaire Corvasal Diltiarem. Cinculation. 1997;95:83-89.

35. Pate RP, Levonen A, Crawford JH, Darley-Usmar VM. Mechanisms of rhe pro- and anti-oxidant mctions of nitric oxide in atherosclerosis [In Process Citation]. Cardiovas Res. 2000;47:465-474.

36. Pratico D, Iuliano L. Mauriello A, Spagnoli L, Lawson JA, Rakach J, Maclouf J, Violi F, FitzGerald GA. Localization of distince $\mathrm{F} 2$-isoprostanes in thuman atheroselerotic lesions. I Chin Invect. $1997 ; 100: 2028-2034$.

37. Parico D, Tangirala RK, Rader DJ, Rokach J, FiczGerald GA. Vitamin Esuppresses isoprostane genetation in viwo and reduces atherosclerosis in ApoE-deficient mice. Nat Med. $1998 ; 4: 1189-1192$.

38. Laursen JB, Somers M, Kurz S, McCann L, Warnhola A, Freeman BA, Tarpey M, Fukai T; Haruson DG. Endothelial regularion of vasomorion in apoE-deficient mice: implications for interactions berween peroxynicrire and tetrahydrobioprerin. Circulation. 2001;103:1282-1288. 
39. Feron $\mathrm{O}$, Dessy $\mathrm{C}$, Moniorte $S$, Desager JP, Balligand JL. Hypercholesterolemia decreases nirric oxide production by promoting the interaction of caveolin and cndorhelial nirric oxide syrathase. J Clin Invest. 1999;103:897-905.

40. Stroes ES, van Faassen EE, Yo M, Marrasek P, Boer P, Govers R, Rabelink TJ. Folic acid revers dysfuncrion of endochelial nitric oxide synthase. Cir Res, 2000;86:1129-1134.

41. Verhaar MC, Wever RM, Kastelein J], van Dam T, Koomans HA, Rabelink TJ. 5-mechylcetrahydrofolate, the active form of folic acid, restores endorhelial funcrion in familial hy. percholesterolemia. Circulation 1998;97:237-241.

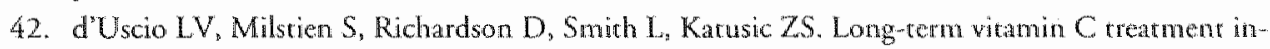
creases vascular terrahydrobiopterin levels and nitric oxide synthase activity. Cor Res. 2003;92:88-95.

43. Zou MH, Shi C, Cohen RA. Oxidarion of the zinc-thiolate complex and uncoupling of endothelial nitric oxide synthase by peroxynitrite. $J$ Clin Intert. 2002;109:817-826. 



\section{General discussion}

\section{INTRODUCTION}

In this thesis, the main hyporhesis tested is that NO inhibits the remodeling response after vascular injury. To achieve this, we characterized endorhelial function and vascular remodeling in response to acute and chronic increased flow in a hyperlipidemic mouse model. Furthermore, we studied the effect of eNOS gene therapy and factors known to enhance eNOS enzyme activity, i.e. folates and estrogen, on vascular remodeling after arterial damage in several mouse strains.

The main findings of these studies are thar:

- hyperlipidemia reduces endothelium-dependent NO-mediated relaxations. We demonstrate mechanistic evidence that vasoconstricting cyclooxygenase products contribute to this impairment in relaxation.

- outward remodeling in response to chronic elevated blood flow is not compromised in mild and severe hyperlipidemia despite indications that acute flow-induced endothelium-dependent dilarion is impaired in severe hyperlipidemia.

- estrogen's protecrive effecr against medial hyperplasia and vascular smoorh muscle cell proliferation in response to mechanical injury are mediated through a NO-dependent pathway. More specifically, we find that regulation of NOS-2 expression plays an important role in the estrogen-mediated effect in mitigating the wound healing response after vascular injury.

- in our vascular gene transfer studies in hyperlipidemic, atherosclerotic mice, we show for the first time that the protective effects of eNOS against neointimal hyperplasia are maintained in a milieu that is conducive to $\mathrm{NOS}$ uncoupling and that is rich in superoxide. The NOS potentiator folic acid, did not significantly enhance this effect. 
Table 1. Overvicw experments flow-related wascular remodeling.

\begin{tabular}{|c|c|c|c|c|}
\hline & & \multicolumn{3}{|c|}{$\begin{array}{l}\text { Chapter 2; cfects hypercholesterolemia on endothelial func } \\
\text { ron, acute flow-induced dilation and fow-related remodeling }\end{array}$} \\
\hline \multicolumn{2}{|l|}{ Srain } & \multicolumn{3}{|c|}{ C57BL/6) (WT)/apoE KO (KO) } \\
\hline \multicolumn{2}{|l|}{ Gender } & \multicolumn{3}{|l|}{ Male } \\
\hline \multicolumn{2}{|l|}{ Age } & \multicolumn{3}{|l|}{6 months } \\
\hline \multicolumn{2}{|l|}{ Dier } & \multicolumn{3}{|c|}{ NC/Westem (D) } \\
\hline \multicolumn{2}{|l|}{ Injury } & \multicolumn{3}{|l|}{ CA liganion } \\
\hline \multicolumn{2}{|l|}{ Groups } & $W T^{\prime N}$ & $W T+D$ & $\mathrm{KO}+\mathrm{D}$ \\
\hline \multirow[t]{3}{*}{ Medial Area (CA) } & UF & $\uparrow$ & $\uparrow$ & $\uparrow$ \\
\hline & NF & ref & $=$ & $\downarrow$ \\
\hline & $\mathrm{HF}$ & $\uparrow$ & $=$ & $=$ \\
\hline \multicolumn{2}{|c|}{ Endothelial dysfunction (CA) } & No & Yes & Yes \\
\hline \multirow[t]{2}{*}{ Pressureadisrension (CA) } & LF & $\downarrow$ & $\downarrow$ & $\downarrow$ \\
\hline & $H F$ & $=$ & $\uparrow$ & $\uparrow$ \\
\hline
\end{tabular}

Scheme overview experiments of chapter 2. ApoE KO, Apolipoprotein E knockout; CA, carotid artery; WT, wild rype; $N C$, nomal chow; D, Western type dier; LF, low flow; $N F$, nomal flow; HF, high flow, ref, used as reference. Media area neasurements is for LF and HF versus sham-operared animass of same group and for NF wersus WT on NC. LF and IF vessels in the pressure-distension measurements are compared to vessels of shammoperited animals.

\section{ENDOTHELIAL DYSFUNCTION, FJOW-INDUCED DILATION AND FLOW-INDUCED VASCULAR REMODELING}

The data of this thesis show that mild and severe hyperlipidemia impair endothelium (ACh)-dependent relaxation (Table 1). This endothelial dysfunction, which is also observed in hyperlipidemic and atherosclerotic human subjects, is associated with a decreased NO bioavailability. Furthermore, the classic endothelial dysfunction observed in the static wire-myograph setup, involved vasoconstricting cyclooxygenase producrs. The impaired endothelial vasorelaxations may be due to decreased expression of eNOS, ${ }^{1}$ a lack of substrate or cofactors for eNOS, ${ }^{2}$ alterations of cellular signaling such that eNOS is not appropriately activated, ${ }^{3}$ accelerated NO degradation by reactive oxygen species (ROS), ${ }^{4}$ and increased levels of asymmetric dimerhylarginine (ADMA). ${ }^{5}$

The classic endothelial dysfunction demonstrated in mild and severe hyperlipidemic mice was not complerely paralleled with the results obtained in a dynamic ser up in which ex-vivo acute flow-induced dilations (FID) were measured. Acute flow-induced dilation was only impaired in the severe hyperlipidemic apoE-deficient mice on a Western diet. The factor(s) causing this impairment could not be elucidated in FID at low flow rates, but at higher flow rates FID was normalized by indomethacin suggesting the involvement of constricting COX-related products. 
Table 2. Effects of COX inhibition on dynamic vascular response under low and high tow conditions in mild and severe hyperlipidemia.

\begin{tabular}{llll} 
& \multicolumn{3}{c}{ FLOW } \\
& low & high \\
\hline HYPERLIPIDEMLA & mild & constriction & no effect \\
& sewere & no effect & dilation
\end{tabular}

In hyperlipidemic mice, indomethacin elicired a response that could be viewed as biphasic (Table 2). Under low flow conditions in mildly hyperlipidemic mice COX-inhibition caused vaso-constriction, indicating a role for vasodilarory COX adducts in dilation under these conditions. Under high flow and severe hyperlipidemia vasoconstrictive COX adducts seem to dictate vasomotion because inhibition of COX enhanced vasodilation. There is controversy over whether chronic medication with COX inhibirors belonging to the group of non-steroid anti-inflammatory drugs (NSAID) increases the risk for ML and cardiac dearh. Several studies have shown an increase, however in a recent meta-analysis this could not be substantiated. ${ }^{6}$ The increased risk has generally been attributed to an increased incidence in coronary thrombosis 7,8 but we show a vasodilatory effect of COX inhibitors under hyperlipidemia and high flow, a situation found in exercising coronary disease patients, that could counteract this thrombosis risk. The dilating vessel could prevent the thrombus from becoming occlusive. Potentially, the net effect of COX inhibitors in coronary patients may depend upon the balance between pro-thrombotic and vasodilatory effects of the NSAID in a particular patient.

When NOS was blocked in addition to COX, vasodilation in response to high flow still occurred in both hyperlipidemic groups. This suggests that other vasodilators contribute to FID as well. A likely candidate could be endorhelial derived hyperpolarizing factor(s) (EDHF) of which the exact nature is not known yet. Also, in the severely hyperlipidemic mice FID at high flow was less pronounced when both enzymes were blocked compared to COX blockade alone. "This may point to some degree of preserved NO generation in the presence of severe dyslipidemia. The observation that even in severelly hyperlipidemic animals, an artery dilates in response to high flow ex vivo, correlates well with the in vivo finding of preserved outward remodeling in response to chronically increased flow in hyperlipidemic animals. The classic endothelial dysfunction as demonstrated in the myograph studies did not predict the remodeling response in vino. The fact that this complex response does not correlate with agonist induced responses in a myograph should not surprise us. Flow-induced remodeling occurs rapidly, i.e within several wecks in animal models, and is age-dependent. ${ }^{9}$ Particularly, endothelial NO seems to be involved, based on studies with inhibitors of endothelial NO synthase (eNOS) ${ }^{10}$ and eNOS knockout mice. "In contrast, this thesis suggests that eNOS-derived NO is not a dominant mediator of arterial remodeling in response to chronic altered blood flow (Chapter 2). Furthermore, several other studies also suggest that the endothe- 

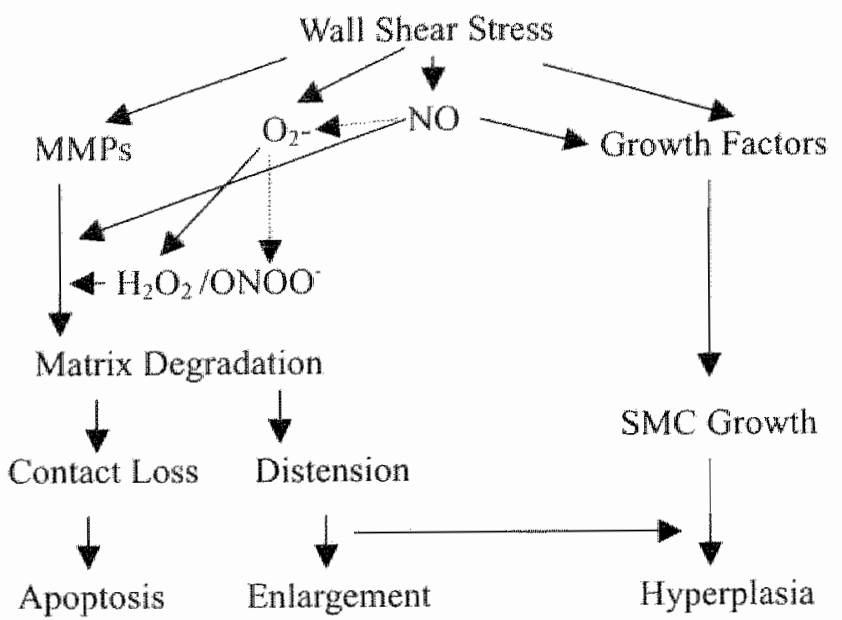

Figure 1. Diagram showing blood flow mediated mechanisms leading to arterial remodeling. Adapted from Lehoux et al. ${ }^{58}$ Dorred arrows indicate the condensation of NO and superoxide to peroxymitrite.

lium ${ }^{12}$ or $\mathrm{NO}^{13}$ are not involved in structural remodeling to increased chronic shear stress. Other proteins that may potentially influence remodeling include matrix metalloproteinases and their inhibitors, ${ }^{14}$ cytokines such as transforming growth factor $\beta\left(\mathrm{TGF}_{\beta}\right)^{15,16}$ growth factors such as platelet derived growth factors (PDGF), ${ }^{17}$ cytoskeletal proteins such as vimentin, ${ }^{12}$ and proteins associated with apoptosis or cell proliferation. 18,19 This thesis does not answer if other NOS isoforms, in particular iNOS, or NO-derived products like peroxynitrite are involved in flow-induced remodeling. In hyperlipidemic mice also used in this thesis, iNOS expression as well as superoxide production ${ }^{20.21}$ are upregulated, leading to the formation of peroxynitrite. iNOS-derived $\mathrm{NO}$, peroxynitrite and other reactive species such as hydrogen peroxide, which can be generated from superoxide through the action of dismutases, can induce metalloproteinase activation ${ }^{14}$ and possibly implicate the induction of growth factor mitogenic activity (Figure 1). Additionally, one report showed endothelium-dependent flow-induced increases in superoxide production ${ }^{22}$ Furthermore, MMP activation is required for adaprive arterial remodeling (fragmentation of internal elastic lamina and arterial enlargement) to occur. These observations are significant since adaptive vascular enlargement and remodeling are known to accompany early human coronary atherosclerosis ${ }^{23}$ and stenosis in several hyperlipidemic mice, ${ }^{24}$ and exaggerated expression of MMPs, in particular MMP-2 and MMP-9, is known to be a marker for aortic aneurysms, 25,26 and could reflect abnormal flow-induced vessel remodeling. The in this thesis observed intact outward vascular remodeling in response to increases in shear stress in mild and severe hyperlipidemia (Chapter 2), with subsequent elevated (flow-dependent) oxidases systems in combination with upregulared expression of inducible NOS, could be the result of MMP activation by $\mathrm{NO}$ /peroxynitrite or other reacrive oxygen species formed in the vessel wall. 
The response to injury after vascular injury is followed by neointimal migration of smooth muscle cells (SMC), SMC proliferation and matrix formation by synthetic state SMC. In carotid, aortic, and iliac arteries of normolipidemic, ovariectomized rabbits, rats, and monkeys, estrogen $\left(\mathrm{E}_{2}\right)$ reduces neointimal and medial growth by $60 \%$ or more after injury to the endothelium by balloon catheter or passage of a fine wire (for review, ${ }^{27}$ ). Estrogen receptor $\alpha(E R \alpha) \mathrm{KO}$ and ER $\beta \mathrm{KO}$ mice have also been used to clarify the roles of ERs in the protective mechanism of $E_{2}$ after vascular injury. The first two such studies, ${ }^{28,} 29$ using a fine wire to denude carotid endothelial cells, demonstrated that $E_{2}$ protects against vascular injury in normolipidemic chimeric $\mathrm{ER} \alpha \mathrm{KO}$ and ER $\beta \mathrm{KO}$ mice. "These findings suggest that ER $\alpha$ and ER $\beta$ are able to complement one another such that each receptor alone is sufficient to mediate the vascular protective effecrs of estrogen, or that the vascular protecrive effects of estrogen are mediated by an ERa/ER $\beta$-independent pathway. In this thesis, $E_{2}$ lost its protection on medial area but not on vascular smooth muscle cell proliferation (VSMC) in ER $\alpha, \beta$ (double) KO mice (Table 3). This raises the questions whether there are ERs other than ER $\alpha$ and ER $\beta$ mediating the observed protective effect of $E_{2}$ on VSMC proliferation, wherher there is a residual function of protein from an ER $\alpha$ splice variant known to be expressed in the parental chimeric $\mathrm{ER} \alpha \mathrm{KO}$ mice ${ }^{30}$ or that this effect is receptor independent. A subsequent study using newly created ERaKO mice in which ER $\alpha$ has been fully disrupted solved this question. ${ }^{31}$ In this study estrogen no longer protected against vascular injury on both medial area and VSMC proliferation, indicating that ER $\alpha$ mediates the protective effects of estrogen.

The atheroprotective effecrs of $\mathrm{E}_{2}$ in the vasculature have been widely attributed to its ability to increase the bioavailability of NO. Physiologic levels of $\mathbb{E}_{2}$ srimulate eNOS mRNA and protein expression through long-term, receptor-dependent mechanisms and upregulate $\mathrm{NOS}$ activity through short-term, nongenomic mechanisms. ${ }^{32}$ Absence of eNOS in apoE KO mice results in hyperrension and increased atherosclerotic lesion size. ${ }^{33}$ In contrast, exogenous delivered $\mathrm{E}_{2}$ to gonadectomized female and male mice deficient in $\mathrm{N} N \mathrm{OS}$ and apoE resulted in reduced lesion size of more than $75 \%$ as compared with control-treated mice deficient of both eNOS and apoE or apoE alone, demonstrating that the atheroprotective effects of exogenous $E_{2}$ do not depend on the presence of eNOS. ${ }^{34}$

In contrast to these observations, we showed that the response to carovid endothelial denudation in normocholesterrolemic mice was inhibited by exogenous $\mathrm{E}_{2}$ and that this protective effect of $\mathrm{E}_{2}$ was completely blocked by systemically administered L-NAME, demonstrating that $\mathrm{E}_{2}$ protection in this model is mediated via a NO-dependent pathway (Table 3 and Chapter 4). A recent study using five inbred strains of mice demonstrated that the genes dictating susceptibility to injury-induced neointimal hyperplasia are distincr from those that determine susceptibility to 


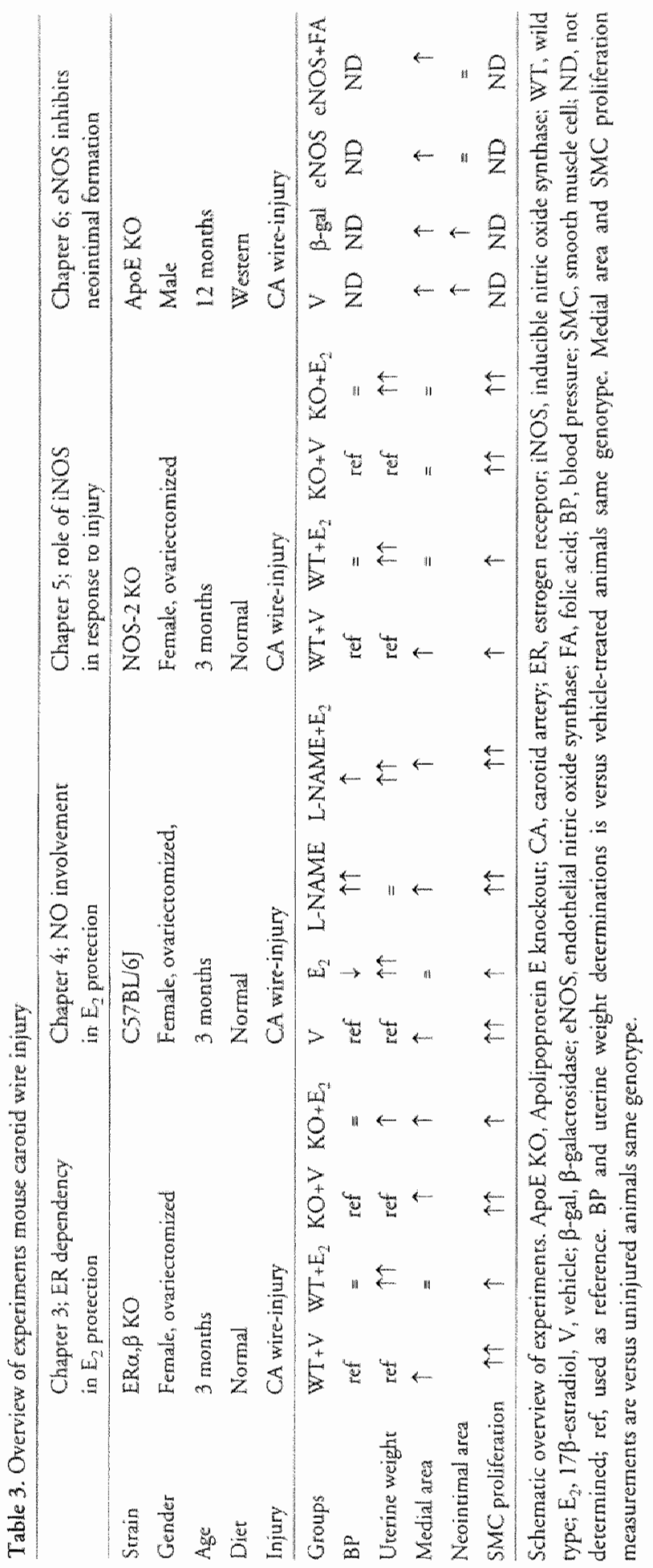


diet-induced atherosclerosis, ${ }^{35}$ which may explain the apparent discrepancy of the $\mathbb{E}_{2}$ protection in normo- and hypercholesterolemic mice.

$E R \alpha$-related genes that might be involved in estrogen's inhibitory effects on the responses to vascular injury include eNOS, iNOS, cyclooxygenase-1, matrix metalloproteinase-2, and estrogen-inhibited genes such as endothelin-1, vascular adhesion molecules, and the angiotensin II receptor AT-1 (review see ref ${ }^{36}$ ). The data of this thesis provide evidence that estrogen's protective effects are mediated by downregulation of injury-induced iNOS expression as the response to injury in vehicle-treated mice deficient of iNOS closely resembled the values of $E_{2}$-treated WT mice (Chapter 5) and $\mathrm{E}_{2}$ treatment did not show any additional effects in iNOS deficient mice (Chapter 5). Although medial area was not increased in response to arterial injury in iNOS deficient mice, medial VSMC proliferation was not completely inhibited by iNOS deficiency (Table 3). This suggests, that other phenomena like extracellular matrix formation ${ }^{31}$ and adventivial myofibroblast migration ${ }^{37}$ to the media are prominent in medial thickening.

\section{THE ROLE OF ENOS IN INJURY-INDUCED VASCUILAR REMODELING IN HYPERLIPIDEMIA}

In hyperlipidemia with elevated vascular superoxide production by increased expression of ROS, NO combines with superoxide to yield peroxynitrite, resulting in decreased bioavailable NO. The formed peroxynitrite oxidizes tetrahydrobiopterin $\left(\mathrm{BH}_{4}\right)$, an essential cofactor for eNOS. Depletion of this cofactor results in uncoupling of eNOS activity and a switch from praduction of $\mathrm{NO}$, from L-arginine, to generation of superoxide. ${ }^{38} \mathrm{~L}-5-\mathrm{MTHF}$, the main active form of folic acid in the circulation, reverses the oxidation of $\mathrm{BH}_{4}$ and restores endothelial function in familial hypercholesterolemia. ${ }^{39}$ In vitro, L-5-MTHF demonstrated to be capable of directly scavenging superoxide, increasing NO production by eNOS and also reducing superoxide generation by eNOS.40 In animal models of arterial injury, exogenous delivery of $\mathrm{NO}$ compounds or enhancement of endogenous $\mathrm{NO}$ production by administration of $\mathrm{L}$-arginine reduces the vascular response. ${ }^{4} 1,42 \operatorname{In}$ this thesis we provide data indicating that local gene delivery of eNOS completely inhibits neointimal remodeling after carotid endothelial denudation in hyperlipidemic mice (Table 3 and Chapter 6). Subsequently, additional effects of L-5-MTHF on neointimal inhibiton by eNOS could not be observed, suggesting that eNOS enzymatic activity does not get uncoupled in hyperlipidemic mice. Surprisingly, eNOS treatment with or without L-5-MTHF administration was not able to inhibit the medial response to injury. This may be in apparent contrast to the other studies described in this thesis in which $N O S$ activity potentiating agents like $E_{2}$ inhibited the medial response to injury (Chapters $3-5$ ). However, $\mathbb{E}_{2}$-induced protection on medial area after vascular injury in iNOS KO mice seemed to be independent of eNOS (Chapter 5). Furthermore, it is possible that eNOS-derived NO in medial 
area of transduced carotid arteries is overwhelmed by superoxide produced by elevated oxidase systems. Hence no effect of NO can be expected. On the luminal border eNOS-derived NO mighr not or not completely be scavenged by superoxide and may therefore exert its protective effect by inhibiting the release of growth factors involved in neointimal formation, like PDGF, or endothelial adhesion molecules like, von Willebrand factor (vWF). Recently, a study reported that an antibody raised against WWF prevented neointimal formation in balloon-injured carotid arteries of normocholesterolemic guinea pigs but could not inhibit cultured VSMC proliferation. 43

\section{CONCLUDING REMARKS}

In the second chapter of this thesis we proved endothelial dysfunction in hyperlipidemia characterized by a diminished ability of the arteries to relax in response to increasing concentrations of acetylcholine, suggesting reduced eNOS enzymaric acriviry. Furthermore, we observed, making use of a more dynamic experimental setring, that acute flow-induced dilations was impaired in severe hyperlipidemic mice. However, in these experiments we could not attribute a prominent role for endothelial-derived $\mathrm{NO}$ in mediating flow-induced dilations. In general, acute flow-induced dilations seemed to inwolve non- NO and cyclooxygenase-related products that may be endothelium-derived hyperpolarizing factor(s). Surprisingly, the observed diminished acrivity of the endothelium to respond to wasodilating substances and mechanical stimuli (shear stress) did not result in impaired outwand arterial remodeling in response to chronic increased shear stress. This suggests that the endothelium, in particular $\mathrm{NOS}$, may not be involved in mediating arterial remodeling in a hyperlipidemic mouse model of chronic elevared shear stress. This does not imply that NO does not play part in vascular remodeling in this model. One of the features of hyperlipidemia is increased expression of superoxide production and elevated production of iNOS-derived NO. Both iNOS-NO or superoxide or the condensarion product of these molecules, peroxynitrite, may facilitate induction of MMP expression. Adaptive vascular remodeling is accompanied with enhanced expression of $\mathrm{MMP}_{\mathrm{s}}$, in particular MMP-2. However, exaggerated induction of MMPs may lead to abnormal vascular remodeling giving rise to incidental aneurysms.

In chapter 3 we show that estrogen protection against vascular damage on medial area is mediated via one of the known estrogen receptors and that this involves $\mathrm{NO}$ synthase(s) (Chapter 4). The 'bad' factor involved in injury-induced medial thickening is iNOS (Chapter 5). It has repeatedly been reported that estrogen induces release of NO via eNOS activation and that this may be a potential pathway in estrogen protection against atherosclerosis and in 'the response to injury' models in mice. The fact that downregulation of INOS by estrogen is involved in estrogen-mediated prorection on medial thickening afrer vascular injury in. 
normocholesterolemic mice (Chapter 5) is in contrast with the findings that blockade of all NOS isoforms, including iNOS, by L-NAME resulted in an increase of medial area in animals treated with estrogen (Chapter 4). The question by which mechanism(s) estrogen regulates iNOS expression remains unanswered. There is little evidence for a direct interaction of estrogen with the iNOS promotor, which lacks estrogen responsive elements. ${ }^{44}$ The question remains whether NOS and/or nNOS is involved primary to iNOS acrivation. As proposed for the effect of estrogens on other molecules of the inflammatory cascade, it is conceivable that iNOS mRNA synthesis is controlled by estrogen by interaction with transcription factors such as nuclear factor-KB. ${ }^{45}$ Furthermore, NO has been shown inducing and stabilizing $I \kappa B \alpha$, which in turn, blocks activation of the proinflammatory transcription factor NF-kB. ${ }^{46,47}$ Furthermore, eNOS-derived NO reduced ischemia-reperfusion injury by inhibiting NF-KB activation in a rabbit heart transplant model. 48 This still does not explain why L-NAME blocked estrogen protection to vascular injury where iNOS-related products are responsible for medial thickening. One explanation may be that L-NAME did not fully inhibit iNOS activiry. Injury-induced iNOS expression may be elevated to such an exrent that L-NAME is not able anymore to completely inhibit iNOS activity or the affinity of L-NAME for iNOS may be lower than for the other NOS isoforms. In contrast, however, L-NAME might have inhibited the activity of eNOS/nNOS protein completely resulting in the activation of $\mathrm{NF}-\kappa \mathrm{B}$ and subsequently $\mathrm{NOS}$ activation. Blocking the rranscription factor $\mathrm{NF}-\mathrm{KB}$ for $\mathrm{NOS}$ by $\mathrm{NOS} / \mathrm{nNOS}$ might be more efficient in inhibiting the response to injury than blocking iNOS protein acrivity by L-NAME.

In chapter 6 we showed that $\mathrm{NOS}$ is involved in inhibiting neointimal formation but was not able to inhibit medial thickening in response to arterial endothelial denudation in male hypercholesterolemic mice. It is not dear if eNOS mediated protecrion acts through (down) regulation of iNOS expression in this model. If that would be the case then one would expect inhibition of medial thickening as well. Nevertheless, eNOS might have down-regulated NF-KB and other factors like, adhesion molecules (VCAM-1, ICAM-1, E-selectin), ${ }^{4 y-51}$ chemotactic agents $(\mathrm{MCP}-1)^{52}$ and thrombogenesis (tissue factor $)^{53}$ involved in neointimal formation on the luminal border, specifically platelets ${ }^{54}$ and monocytes ${ }^{55}$ but might not have been able to modulate NF- $\mathrm{KB}$ induced expression of iNOS in the inner layers of the media where the oxidative stress is higher.

\section{FUTURE ASPECTS}

Vascular remodeling after PTCA is characterized by neointimal formation leading to lumen loss. In this thesis we showed that local eNOS gene transfer inhibits arterial injury-induced neointimal growth, but not medial thickening, in a hyperlipidemic mouse. 
Recently, the implantation of stents to prewent constrictive remodeling of the vessel wall has become the most common coronary percutaneous revascularization procedure. e-NOS gene transfer may prove very effective in the prevention of in-stent restenosis which is characterized primarily by a proliferative response as inward remodeling is being prevented by the rigidity of the stent. Moreover, stents can be implemented as delivery platforms for genes or drugs. The first multicenter trial with stents coated with an immunosuppressant completely prevented angiographic restenosis at 6 months follow up. ${ }^{56}$ Other drug-eluting stent systems like vascular endothelial growth factor and $17-\beta$ estradiol are currently under evaluation. 57

Furure experiments are warranted to examine whether eNOS gene delivery at site may prove useful against restrictive remodeling in other animal species and humans.

\section{REFERENCES}

1. Wilcox JN, Subramanian RR, Sundell CL, Tracey WR, Pollock JS, Harrison DG, Marsden PA. Expression of multiple isoforms of nitric oxide synthase in normal and atheroscleroric vessels. Artevionder Thromb Vasc Biol. 1997;17:2479-2488.

2. Pou S, Pou W, Bredt D, Snyder S, Rosen G. Generation of superoxide by purified brain nitric oxide synthase. J. Biol. Chem. 1992;267:24173-24176.

3. Shimokawa $H$, Flavahan NA, Vanhoutte PM. Loss of endothelial pertussis toxin-sensitive G prorein function in atherosclerotic porcine coronary arteries. Circulation. 1991:83:652-660.

4. Cail H. Harrison DG. Endothelial dysfunction in cardiovascular diseases the role of oxidant stress. Cive Res. 2000;87:840-844.

5. Jang J. Ho H-KV, Kwan HH, Fajardo LF, Cooke JP. Angiogenesis Is Impaired by Hypercholesrerolemia : Role of Asymmetric Dimethylarginine. Circhlation. 2000;102:1414-1419.

6. White WB, Faich $G_{\text {. B }}$ Borer JS, Makuch RW, Cardiovascular thromboric ewents in arthritus trials of the cyclooxygenase-2 inhibitor celecoxib. The Amexican foum of Cardiology. 2003;92:411-418.

7. Mulkerjee D, Nissen SE, Topol EJ. Risk of cardiovascular events associated with selective COX-2 inhibitors. Jama. 2001:286:954-959.

B. Konstam MA, Weir MR, Reicin A, Shapiro D, Sperling RS, Barr E, Gertz BJ. Cardiovasculat thrombotic events in controlled, clinical rrals of tofecoxib. Circulation. 2001;104:2280-2288.

9. Miyashiro JK, Poppa V, Berk BC. Flow-induced vascular remodeling in the rat carotid artery diminishes with age Circ Res. 1997,81:311-319.

10. Tronc F, Wassef M, Esposito B. Hention D. Glagov S. Tedgui A. Role of NO in flow-induced remodeling of we rabbit common carotid artery. Arteriascler Thromb Vasc Biol. 1996;16:1256-1262.

11. Rudic RD, Shesely EG, Maeda N, Smithies O, Segal SS, Sessa WC. Direct evidence for the importance of cndothelium-derived nitric oxide in wascular remodeling. J Clin Imvest. 1998;101:731-736.

12. Schiffers PM. Henrion D, Boulanger CM, Colucci-Guyon E, Langa-Vuves F, van Essen H, Fazzi GE, Levy Bl, De Mcy JG. Alered Alow-induced arterial remodeling in vimentin-deficient mice. Arterioster Thromb Vac Biol. 2000;20:611-616. 
13. Ceiler DL, De Mey JG. Chronic N(G)-nitrow-arginine methyl ester treatment does not prevent flow-induced remodeling in mesenteric feed arteries and arcading artetioles [In Process Cikation]. Arteriosicler Thromb Vasc Biol 2000;20:2057-2063.

14. Galis ZS, Khatri J]. Matrix metalloproteinases in vascular remodeling and arherogenesis: the good, the bad, and che ugly. Cin Res. 2002;90:251-262.

15. Ohno M, Cooke JP, Dzau VJ, Gibbons GH. Fluid shear stress induces endothelial ransforming growth factor bera-1 transcription and production. Modulation by potassium channel blockade J Clin Invest. 1995:95:1363-1369.

16. Smich JD, Bryanr SR, Couper LL, Vary CP, Gotwals PJ, Koteliansky VE, Lindner V. Soluble transforming growth facror-beta rype II receptor inhibits negarive remodeling, fibroblast transdifferentiation, and intimal lesion formation but not endothelial growth. Cin Res. $1999 ; 84: 1212-1222$

17. Mondy JS, Lindner V, Miyashiro JK, Berk BC, Dean RH, Geary RL. Platelet-derived growth facror ligand and receptor expression in response to altered blood fow in viwo. Car Res. $1997 ; 81: 320-327$.

18. Cho A, Mitchell L, Koopmans D, Langitle BL. Effects of changes in blood flow rate on cell dcath and cell proliferation in carotid arteries of immature rabits. Cinc Res. 1997:81:328-337.

19. Langille BL. Arrerial remodeling: relation to hemodynamics. Cat f Physol Phamanacol 1996:74: $834-841$.

20. Kuhlencordi PJ, Chen J, Han F, Astern J, Huang P'L. Genetic deficiency of inducible nitric oxide synthase reduces atherosclerosis and lowers plasma lipid peroxides in apolipoprotein Emknockour: mice. Circulation. 2001;103:3099-3104.

21. d'Uscio LV, Milstien $S$, Richardson D, Smith $L$, Katusic ZS. Long-term vitamin C rreatment increases vascular tetrahydrobiopterin levels and nitric oxide synthase activity. Ciw Res. 2003;92: $88-95$.

22. Laurindo FR, Pedro Mde A, Barbeiro HV, Pileggi F; Carwalho MH, Augusto O, da Luz PL. Vascular free radical release. Ex vivo and in wivo evidence for a flowndependen endorthelial mechanism. Cire Res. 1994;74:700-709.

23. Glagow S, Weisenberg E, Zarins CK. Stankunavicius $\mathbb{R}$, Koletis GJ. Compensatory enlargenent of human atherosclerotic coronary arteries. N Engl/ Med. 1987:316:1371-1375.

24. Lutgens E, de Muinck ED. Heeneman S, Damen MJ. Compensatory enlargement and stenosis

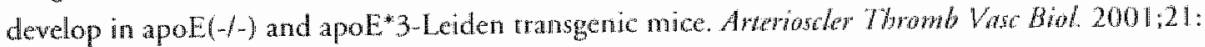
1359-1365.

25. Deng GG, Martin-McNulry B, Sukovich DA, Freay A. Halks-Miller M, Thinnes T, Loskutoff DJ, Carmeliet P. Dote WP, Wang X'X. Urokinase-Type Plasminogen Activator Plays a Critical Role in Angiotensin II-Induced Abdominal Aortic Ancurysm. Cire Res. 2003;92:510-517.

26. Goodall S, Crowther M, Hemingway DM, Bell PR. Thompson MM. Ubiquitous elevarion of ma. trix metalloproteinase-2 expression in the vasculature of parients with abdowinal ancurysms. Cow culation. 2001:104:304-309.

27. Holm P. Effect of estrogen on development of atherosclerosis. A teview of experimental animal studies. Don Med Bull 2001:48:146-160. 


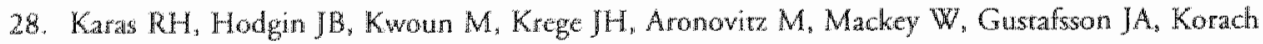
KS, Smithies O, Mendelsoln ME. Estrogen inhibits the vascular injury response in estrogen receptor beta-deficient female mice. Proc Nat Acad Sci U SA. 1999;96:15133-15136.

29. Iafrati MD, Karas RH, Aronovitz M, Kim S, Sullivan TR, Jr, Lubahn DB, O. Donnell TE J, Koradn KS, Mendelsohn ME. Estrogen intibirs the wascular injury response in estrogen receptor alpha-deffient mice. Nat Med 1997:3:545-548.

30. Couse JF, Curris SW, Washburn TF, Lindzey J, Golding TS, Lubahn DB, Smithies O, Korach KS. Analysis of transcription and strogen insensitivicy in the female mouse after targeted distuprion of the estrogen receptor genc. Mol Endocrinol. 1995;9:1441-1454.

31. Pare G, Krust A, Karas RH, Dupont S, Aronovizz M, Chambon P, Mendelsohn ME Estrogen Receptor-alpha Mediates the Protective Effects of Estrogen Against Vascular Injury. Circ Res. 2002;90:1087-1092.

32. Mendelsohn ME, Karas RH. The prorective effects of estrogen on the cardiovascular system. $N$ Lingl J Med 1999;340:1801-1811.

33. Knowles JW, Reddick RL, Jennerre JC, Shesely EG, Smithies O, Maeda N. Enhanced atherosclerosis and kidney dysfunction in $\mathrm{NOS}(-/)$ Apoe(-/-) mice are amelionated by enalapril treatment. $)$ Clin Intest. 2000;105 Feb:451-458.

34. Hodgin JB, Knowles JW, Kim HS, Smithies O, Maeda N. Interactions between endothelial nitric oxide synthase and sex hormones in vascular protection in mice. J Clin Intest. 2002;109:541-548.

35. Kuhel DG, Zhu B, Witte DP, Hui DY. Distinction in Generic Determinatats for Injury-Induced Neointimal Hyperplasia and Diet-Induced Atherosclerosis in Inbred Mice. Arterioscler Thromb Vasc Biol. 2002;22:955-960.

36. Mendelsohn ME, Karas RH. Estrogen and the blood vessel wall. Curr Opin Cardiol. 1994:9:619-626.

37. Zalewski A, Shi Y. Vascular Myofibroblasts : Lessons From Coronary Repair and Remodeling. Arterioscler Thromb Vasc Biol 1997;17:417-422.

38. Vasquez-Vivar J, Kalyanaranan B, Marcasek P, Hogg N, Masters BS, Karoui H, Tordo P, Pritchard KA, Jr. Superoxide generarion by endothelial nitric oxide synthase. the influence of coFactors. Pror NaW Acad Sai US A. 1998:95:9220-9225.

39. Vethat MC, Wever RM, Kastelein J, van Dam T, Koomans HA, Rabelink T]. 5-methyltetrahydrofolate, the active form of folic acid, restores endothelial function in familial hypercholesrerolemia. Circulation. 1998;97:237-241.

40. Stroes FS, van Faassen EE, Yo M, Martasek P, Boer P. Govers R, Rabelink TJ. Folic acid reverts dysfuncrion of endothelial nitric oxide synthase. Corc Res. 2000;86:1129-1134.

41. Kalinowski M. Alfke H. Bergen S, Klose KJ, Barry JJ, Wagner HJ. Compararive trial of local. pharmacotherapy with L-arginine, Ir-hirudin, and molsidomine to reduce restenos is after balloon angroplasty of stenotic rabbir iliac arteries. Radiology. 2001:219:716-723.

42. Maffa P, Ianaro A, Sorrentino R, Lippolis L, Maiello FM, del Soldato R, lalenti A, Cirino G. Beneficial effects of NO-releasing derivative of flubiprofen (HCT-1026) in rar nodel of vascular injury and restenosis. Arterioscler Thrombl Vasc Biol. 2002:22:263-267.

43. Kageyama S. Yamamoro H, Yoshimoto R. Anti-Human von Willebrand Factor Monoclonal Antibody AWW-2 Prevents Thrombus Deposition and Neointima Formation After Balloon Injury in Guinea Pigs. Atrerioscler Thromb Vas Biol. 2000:20:2303-2308. 
44. Miller L, Alley EW. Murphy WJ, Russell SW. Hunt JS. Progesterone inhibits inducible nitric onide synthase gene expression and nitric oxide production in murine nacrophages. I Lenkor Brol $1996,59: 442-450$.

45. Xie QW, Kashiwabara $Y$, Nathan C. Role of transcription factor NF-kappa B/Rel in induction of nitric oxide synthase. J Biol Chem. 1994:269:4705-4708.

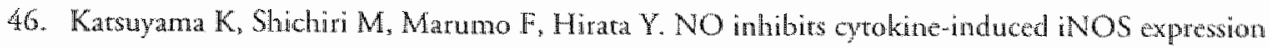
and NF-kappaB activation by interfering with phosphorylation and degradarion of IkappaB-alpha. Arterioscler Thromb Vasc Biol 1998;18:1796-1802.

47. Peng HB, Libby P, Liao JK. Induction and stabilization of I kappa B alpha by nitric oxide mediares inhibition of NF-kappa B. J Biol Chem. 1995:270:14214-14219.

48. Iwara A, Sai $S$, Nitta $Y$, Chen $M$, de Fries-Hallstrand $R$, Dalesandro J, Thomas $R$, Allen MD. Liposome-Mediated Gene Transfection of Endothelial Nirric Oxide Synthase Reduces Endothelial Accivation and Leukocye Infiltarion in Transplanted Hears. Cimalatom 2001;103: 2753-2759.

49. Graber N, Gopal TV, Wilson D, Beall LD, Polte T, Newnan W. T cells bind to cyrokine-acrivated endothelial cells via a novel, inducible sialoglycoprotein and endothelial leukocyte adhesion molecule-1.J/ Immunot. 1990;145:819-830.

50. Dustin ML, Rothlein $R$, Bhan AK, Dinarella CA, Springer TA. Inducrion by IL 1 and interferon-gamma: tissue distriburion, biochemisrcy, and funcrion of a natural adherence moleculie (ICAM-1). J Immunol. 1986;137:245-254.

51. Carlos TM, Schwarz BR, Kowach NL, Yee E, Rosa M, Osbom L, Chi-Rosso G, Newman B, Lobb $R$, Rosso $M$, et al. Vascular cell adhesion molecule-1 mediates lymphocyte adherence ro cytokine-activated cultured human endorhelial cells. Blood. 1990;76:965-970.

52. Yla-Hertuala S, Lipton BA, Rosenfeld ME, Sarkioja T, Yoshimura T, Leonard EJ, Wirzrum JL, Steinberg D. Expression of nonocyte chemoattractan protein 1 in macrophagerich areas of human and rabbit atherosclerotic lesions. Proc Not Arad Sci U S A. 1991;88:5252-5256.

53. Mackman N. Brand K, Edgington TS. Lipopolysacharide-mediated ranscriprional acrivation of rhe human tissue factor gere in THP-1 monocytic cells requires both activator protein I and nuclear factor kappa B binding sires. J Exp Med. 1901;174:1517-1526.

54. Liu F, Morris S, Epps J, Carroll R. Demonstration of an activation regulated NV kappa B/1-kappaBalpha complex in human plarelers. Thomb Res. 2002;106:199-203.

55. Brand K, Eisele T, Kreusel U, Page M, Page S, Has M, Gerling A, Kalrschmidt C, Neumann FJ, Mackman N, Baeurele PA, Walli AK, Neumeier D. Dysregulation of monocyric nuclear factor-kappat B by oxidized low-densiny lipoprotein. Arteriosch Throwh Vasc Biol. 1997:17:1901-1909.

56. Morice MC, Sernys PW, Sousat JE, Fajader J, Ban Hayash E, Perin M, Colombo A, Schuler G, Barragan P, Guagliumi G. Molnar F, Falotico R. A randomized comparison of a sirolimus eluning stent with a standard stent for coronary revascularization. N Engl/ Med. 2002:346:1773-1780.

57. Sousa JE, Serruys PW, Costa MA. New frontiers in caudiologyo drug-eluting stents: Part II. Circhlation. 2003;107:2383-2389.

58. Lehoux S, Tronc F", Tedgui A. Mechanisms of blood flow-induced wasculat enlargement. Biombology. 2002;39:319-324. 



\section{Summary}

Vascular endochelial cells are crucial in preserving the patency of blood vessels. Nitric oxide (NO), is consriturively produced by endothelial cells and is an important secreted factor and has a prominent role in the maintenance of function and anatomy of the vessel and is considered as an atheroprotective molecule.

A hall mark of endothelial dysfunction is the loss of atheroprotective properties of the endothelial lining. Chronic endothelial dysfunction will result in arherosclerotic plaque formation that leads to a lumen-narrowing stenosis when outward remodeling of the vessel fails to compensate for vessel wall thickening. Restenosis, the pathological remodeling of a vessel, after coronary angioplasty in order to reopen an artery after initial lumen-narrowing stenosis, is caused by endorhelial damage and leads to a recurrent narrowing of the vessel, characterized by neointimal growth, in $30-40 \%$ of all patients undergoing angioplasty. Less production of NO by the damaged and impaired functioning endothelial cells may be the cause of restenosis which makes $\mathrm{NO}$ an interesting potential target for the prevention of restenosis. This thesis studies the role of NO in vascular remodeling in mice with damaged endothelial cells and/or impaired endorhelial function.

Diet-induced hypercholesterolemia results in impaired function of endothelial cells that is characterized by less bioavailable NO. Changes in blood flow drive both acute and long term compensatory responses that eventually result in normalization of wall shear stress. In order to accommodate these responses endothelial cells produce substances like NO. Despite observed endothelial dysfunction in hypercholesterolemic mice ourward vascular remodeling was nor impaired ro chronic increases of blood flow, questioning the role of the endorhelium in this phenemenon.

Estrogen protects against heart and coronary diseases and inhibits the vascular response to injury. In this thesis data are provided showing that estrogen's protection is NO dependent. The healthy endothelium produces small quantities of $\mathrm{NO}$, but immense production of $\mathrm{NO}$ is produced by other cells, only after the expression of an inducible nitric oxide synthase (iNOS). This thesis proves that estrogen modulates NOS expression and that this causes estrogen protection against vascular damage in normocholesterolemic mice. 
One of the features of hypercholesterolemia is a reduced activity of the enzyme, endothelial NO synthase ( $N O S$ ), in which both less $N O$ and simultanously superoxide is produced that combines with $\mathrm{NO}$ to the powerful oxidizing molecule, peroxynitrite. In this thesis we show that neointimal growth can be inhibited by local gene transfer of $\mathrm{NOS}$ after vascular damage in hypercholesterolemic mice.

The data of this thesis provide several possible therapies against the parhological responses to vascular injury. Special balloon carheters introducing eNOS gene immediately after angioplasty or coated stents eluting $\mathrm{NO}$ or estrogen may serve as potential therapies against restenosis. 


\section{Samenvatting}

De endotheelcellen van bloedvaten zijn van essenticel belang in de homeostase van bloedvaren. Stikstofoxide (NO), dat door de endotheelcellen continu word aangemaakt, speelt een belangrijke rol in her behoud van een normale functie en vaatwandstructuur van bloedvaten.

Restenose, de vasculaire remodulering van een bloedvat, treedt op in 30-40\% van de gevallen na een zogenaamde 'dotterprocedure' (ballondilatatie) of stentimplantatie waarbij de lumenvernauwende stenose wordt opgeheven maar ook tevens het endorheel wordt beschadigd en ten gevolge hiervan het vat door neointimale ingroei opnieuw dichtslibt. Verminderde NO productie door beschadigde en/of slecht functionerende endotheelcellen ligt mogelijk hieraan ten grondslag en herstel van $\mathrm{NO}$ productie kan dus een mogelijke therapie zijn om deze vorm van vasculaire remodulering regen te gaan. In dit proefschrift wordr bestudeerd wat de rol is van $\mathrm{NO}$ in vasculaire remodulering in muizen met beschadigd endotheel en/of verslecllterde endotheelfunctie.

Verhoogde cholesterolspiegels in het bloed door een vetrijk dicet leidt tot een verslechterde endotheelfunctie gekenmerkt door verminderde biologische beschikbaarheid van NO. Verhoogde bloedsnelheid door een bloedvat leidt acuut tor her uitzetten van her bloedvat en een chronische blootstelling aan verhoogde bloedsnelheid leidt tot structurele veranderingen in de vaatwand om zo de door her bloed gegenereerde krachten op de endotheelcellen en vatwand te normaliseren. Hierbij wordt door het endorheel stoffen geproduceerd zoals $\mathrm{NO}$ om deze anpassing te faciliteren. Ondanks de verslechterde aangeroonde endotheel funcrie in hypercholesterolemische muizen zijn deze toch in staat om hun vaawandstructuur aan te passen dan veranderde bloedperfusie omstandigheden, waarbij de rol van her endorheel in deze ter discussie gesteld kan worden.

Oestrogenen hebben een beschermende functie tegen hart en vaatziekten en inhiberen de parhologische vaatwandremodulering t.g.v. endotheelbeschadiging. De resultaten van dit proefschrift laten zien dat NO een rol speelt in deze bescherming. NO wordt door endotheelcellen in kleine hoevectheden geproduceerd, maar kan ook in grote hoeveelheden door andere cellen worden a angemaakt, alleen nadar cen bepaald enzym, "induceerbaar NO synthase" (iNOS), tot expressie is gekomen 
onder invloed van bepaalde prikkels waaronder ontstekingsreacties. In dit proefschrift wordt aangetoond dat oestrogenen de expressie van INOS negatief beinvloeden en dat dit zorgt voor oestrogeenprotectie ten gevolge van vaatwandbeschadiging in normocholesterolemische muizen.

Hypercholesterolemie wordt gekenmerkt door een verminderde activiteit van het enzym, endotheliaal NO synthase (eNOS), waardoor naast (verminderde) NO productie ook superoxide wordt geproduceerd dat vervolgens met $\mathrm{NO}$ reageert tot het schadelijke peroxynitriet. In dit proefschrift laten we zien dat de neointimale groei van de vaatwand tegengegaan kan worden met behulp van gentherapie waarbij het NOS gen lokaal wordt toegediend na vaatwandbeschadiging in hypercholesterolemische muizen.

Dit proefschrift biedt verscheidene mogelijke therapieën aan om de pathologische remodulering, restenose, die optreedt na vaatwandbeschadiging t.g.v. een procedurele ingreep om her vat te heropenen te behandelen. Te denken valt aan applicatiemethoden waarbij het eNOS gen bij ballondilatatie met een speciale cartheter lokaal toegediend kan worden en of gecoate stents die $\mathrm{NO}$ of oestrogenen vrijgeven. 


\section{Dankwoord}

De haalbaarheid van een promotieonderzoek hangt niet alleen af van de promovendus maar ook van de direct betrokkenen. Bij deze wil ik hen bedanken.

Op de allereerste plaats Professor Wellens. Pas een aantal maanden na mijn aanstelling werd mij duidelijk gemaakt dat mijn promotor een zeer eminent cardioloog was. Het streelt dan ook mijn ego om onder Uw toezicht te mogen promoveren. $U$ heeft voor mij verscheidene malen deuren geopend om goed onderzoek te kunnen uitvoeren. U nam deze taak dan ook zeer letrerlijk; ik zal nooit vergeten dat $U$ voor mij de tussendeuren opende toen $U$ mij zag aankomen lopen in de gang. Artentheid naar anderen toe is $U$ niet vreemd en dat maakt $U$ als persoon, naast een fenomenaal klinisch onderzoeker, extra groot. Ook Uw enthousiasme in het me steunen van wetenschappelijk onderzoek doen, heeft extra motiverend gewerkt.

Professor Mat Daemen. Ik voelde me al meteen thuis in je directe manier van handelen. Gedurende het promotierraject hebben we elkaar voornamelijk tijdens congressen en promoties gezien waar dan even de stand van zaken werd doorgesproken. Zo ook tijdens her tor standkomen wan het proefschrift. Ik wilde promoveren en dus kwam ik naar jou toe met deze boodschap om dat in gang te zerten. Jouw begeleiding gedurende het promotietraject is altijd zeer efficiënt geweest.

Dr. Ebo de Muinck. Ik wist natuurlijk bij aanvaarding van dit promotieonderzoek dat jij als praktiserend interventiecardioloog mij niet in het lab kon begeleiden en dat ik mijn weg zelf moest vinden. Gelukkig wist je mij altijd goed te vertellen waar en bij wie ik moest wezen om de experimenten te kunnen uitvoeren. De rest kwam vanzelf. Het vertalen van experimentele data in schrift en in orale presentaties is wat ik in je zeer bewonder. Ondanks dat je in Nederland een nieuwe groep aan het opbouwen was, heb je toch je heil gekozen voor het land met de onbegrensde mogelijkheden. Ik hoop dat dit ook zal gelden voor je onderzoek aldaar.

Professor Jo De Mey. Als extern verbonden aan dit promoticonderzoek, maar in praktische zin zeer betrokken bij her onderzoek dat ik in Nederland heb uitgevoerd. Jij bent een van de weinige profs die nog altijd in her lab terug te vinden is, renminste als de tijd het je toelaat. Ideeën die bij je opdoemden naar aanleiding van het lezen van een artikel, werden gelijk in de praktijk (lees myograaf) getoetsr; cen wetenschapper 'pur sang'. 
Dear Mike and Dick. You both made it possible for me to work in your lab. More than half of the work presented in this thesis came from mine one year stay at your magnificant institute. You made me feel very comfortable in your lab and your scientific guidance during my stay has developed me to become what I am right now. I have deep appreciation for how you both are running your institute and wish you all the luck you will need.

Mark Aronovitz. You learned me the thing! It's clear that you have golden hands when it comes to surgical procedures and that you have good taste when it comes to beer. Budweiser sucks! You, Gary and Kim were there when I showed you that I have golden hands as well, resulting in the "Dutch Touch". I will make my fortune some day!

Gary Pare. It must be in your name (my brother's name is pronounced like yours), because you were like a brother to me in Boston. We have many things in common. Well, if you don't succeed in being a fine vascular surgeon you can always go back being a scientist; many papers with your name on it have been published in your short practical stay at the MCRI!

Serban, my fellow European and dear friend. They say that good scientists know how to cook. Then you must be an excellent scientist! With you I explored the Asian food court in Chinatown; I even survived. Ding Ho's! I hope that you and your family will do great in the land of Uncle Sam.

Xander Wehrens (MD, PhD), amice, inmiddels ben je al een zeer gerespecteerd wetenschapper, Salsa-koning en een van mijn groorste vrienden sinds ik regelijkertijd met jou ben begonnen in Maastricht.

Dennis, voor intimi Sjaan. Wij hebben de afgelopen jaren de Nederlandse taal werrijkt van neologieën en nieuwe spreuken. Het wordt weer eens tijd dat ik wat vaker naar de Domstad ga.

Tij! (Klakske), gij zijt de slimste Belg van heel Limburg! Het promoveren kan een lijdensweg zijn, maar jij weet eigenhandig de finish te halen binnenkort. Luctor et emergo!

Mijn paranimfen. Sebaske, mijn grote, kleine vriend. Inmiddels ben jij ook begonnen aan wat ik nu juist heb afgesloten. Als ik ergens zeker van ben dan is het dat jij over 4 jaar ook je bedankberuiging zal schrijven. Sinds Unitas hebben we elkaar nooit meer uit her oog verloren en dat zal alrijd zo blijven.

Rob. Uiteindelijk, na 2 jaar, kwam thet er dan toch van zoals het in het promotie-onderzoeksproject stond beschreven; samenwerking met de nog aan te stellen AiO van de Farmacologie op het gebied van flow-remodeling. In begin was ik bang dat mijn werktempo je zou overschaduwen, maar gelukkig ben je ook uit het goede hout gesneden. Binnenkort zal jij ook doctoreren.

Professor Mark Post. Het stuivertje wisselen met Ebo leidde ertoe dat je Ebo's groepje in de schoot geworpen kreeg. Het is hier in het Limburgse toch allemaal wat anders dan in de V.S. dus zal de Limburgse Universiteit zich moeten aanpassen aan jouw persistentie en directe manier van communicarie. Eerder dan verwacht kon je al iemand aannemen op pre-klinisch dierexperimenteel onderzoek en diegene was ik. 
In korte tijd heb je me moeten klaarstomen in de klinisch cardiologische vaardigheden en operatie rechnieken op grote proefdieren. Bedankt voor alle steun en geduld.

Verder wil ik iedereen van de capaciteitsgroepen Cardiologie, Pathologie en Farmacologie \& Toxicologie bedanken die direct of indirect hebben bijgedragen aan het tot standkomen van dic proefschrift.

De allergrootste fans zijn natuurlijk mijn directe familie. Pa en ma, met rrotsheid hebben jullie mijn levenswandel gadegeslagen en me gesteund in al mijn beslissingen, ondanks bedenkingen hier en daar. $\mathrm{Nu}$ is de allerhoogste bestudeerbare ritel gehaald. Pa zal apetrots op me zijn! 



\section{Curriculum Vitae}

Henny Schulten was born May 14 th 1969 in Tilburg, the Netherlands. After passing the VWO (Atheneum-B) exam at the Theresia Lyceum in Tilburg, he studied Medical Biology (Faculty of Medicine) at the University of Utrecht. After graduation, he started a PhD project in the department of Cardiology ar the University of Maastricht to study the role of nitric oxide in arterial remodeling after vascular damage. During this PhD project he worked for one year in the laboratory of Dr. Mendelsohn in Boston, United States (Molecular Cardiology Research Institute, Tufts University). Since 2003 he has been working as a post-doc for the group of Mark J. Post in the department of Physiology at the University of Maastricht.

Henny Schulten werd geboren op 14 mei 1969 te Tilburg, Nederland. Na her behalen van zijn VWO (Atheneum-B) diploma aan het Theresia Lyceum te Tilburg werd de studie Medische Biologie aan de Faculteit Geneeskunde van de Universiteit Urrecht aangevangen. In 1998 werd begonnen met het promotieonderzoek naar de rol stikstofoxide in vasculaire remodelering na vaatwandschade bij de capacireitsgroep Cardiologie an de Universiteir van Maastricht. Tijdens zijn promotieonderzoek heeft hij voor een jaar gewerkt in het laboratorium van Dr. Mendelsohn te Boston, de Verenigde Staten (Molecular Cardiology Research Institute, Tufts University). Sinds 2003 is hij werkzaam als post-doc in de groep wan Mark Post bij de capaciteitsgroep Fysiologie aan de Universiteit van Maastricht. 



\section{List of publications}

M. Mulder, A. Blokland, D-J. Van den Berg, H. Schuleen, A. Bakker, D. Terwel, W. Honig, E. de Kloet, L. Havekes, H. Steinbusch, E. de Lange (2001). Apolipoprotein E protects against neuropathology induced by a high-fat dier and maintains the inrergrity of the blood-brain batriat during aging. Laboratory Invescigarion 81: 953-60.

R. Karas, H. Schulten, G. Pare, M. Aronovitz, C. Ohlson, J-A. Gustafsson, M. Mendelsohn (2001). Effects of estrogen on the vascullar injury response in the estrogen receptor $\alpha, \beta$ (double) knockour mice. Circ. Res. 89: 534-39.

H. Schulten, R. Hilgers, P. Sehiffers, A. Strzelecka, G. Fazzi, E. de Muinck, J. De Mey. Reduced endothelium-dependent relaxations and impaired acute flow-induced vasodilation do not compromise chronic flow-related arterial remodeling in hyperlipidemic mice. (submitred)

H. Schulten, R. Karas, M. Aronowitz, G. Pare, F. Celestin, E. de Muinck, M. Mendelsohn. Nitric oxide synthase(s) mediate the vascular protective effect of estrogen in the mouse carotid artery injury model. (submitted)

D. Neu, H. Schulten, M. Aronovicz, IF. Celestin, R. Karas, M. Mendelsohn. Disruption of the iNOS gene abolishes estrogen-mediated protecrion against vascular injury in mice. (submitred)

H. Schulten, B. Schrans-Stassen, D. Sonnemans, P. Schiffers, M. Post, E. de Muinck. Endotholial nicric oxide synthase inhibits neointimal hyperplasia after vascular injury in thyperlipidenaic mice. (sultomitred) 